
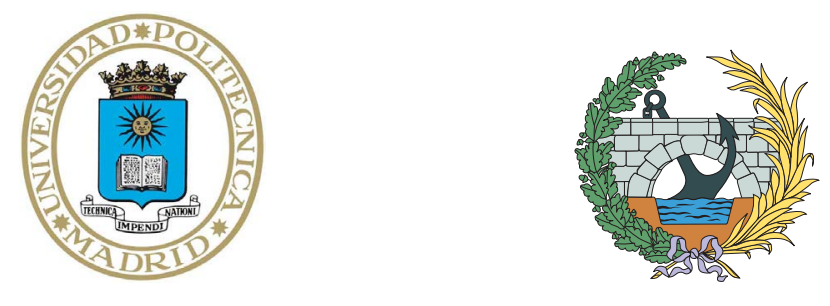

Universidad Politécnica de Madrid

Escuela Técnica Superior de Ingenieros de Caminos, Canales y Puertos

\title{
Mechanical Behaviour and Simulation of Alloys Used in Jet Engines
}

Tesis doctoral

Rafael Sancho Cadenas

Ingeniero de Materiales

2020 


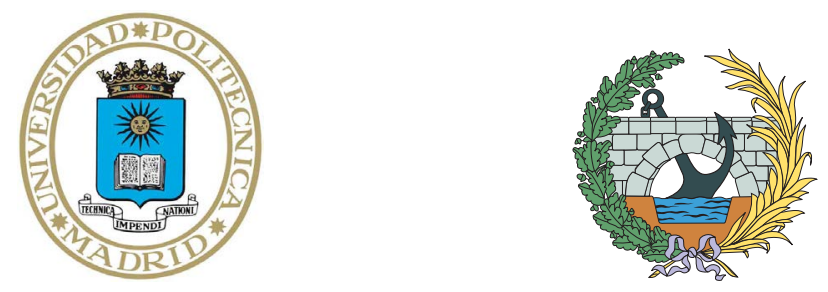

Departamento de Ciencia de Materiales

Escuela Técnica Superior de Ingenieros de

Caminos, Canales y Puertos

Universidad Politécnica de Madrid

\title{
Mechanical Behaviour and Simulation of Alloys Used in Jet Engines
}

\author{
Tesis doctoral
}

Rafael Sancho Cadenas

Ingeniero de Materiales

Director:

Francisco Gálvez Díaz-Rubio

Dr. Ingeniero Aeronáutico

Catedrático de Universidad

2020 

A mis padres 


\section{Contents}

$\begin{array}{ll}\text { Agradecimientos } & \text { V }\end{array}$

$\begin{array}{lll}\text { Abstract } & \text { VII }\end{array}$

$\begin{array}{lll}\text { Abbreviations } & \text { IX }\end{array}$

$\begin{array}{lll}\text { Notation } & \text { XI }\end{array}$

1 Introduction and Objectives $\quad 1$

1.1 Introduction . . . . . . . . . . . . . . . . . . . . . . 1

1.2 Motivation and objectives ................ 5

2 State of the art $\quad 9$

2.1 Introduction to plastic deformation . . . . . . . . . . . . . . 9

2.2 Computational plasticity in the continuum framework . . . . . . 12

2.2.1 Macroscopic plasticity . . . . . . . . . . . . . 13

2.2.2 Crystal plasticity . . . . . . . . . . . . . . . 14

2.2.3 Polycrystal homogenization . . . . . . . . . . 16

2.3 The Johnson-Cook model _. . . . . . . . . . . . . . . . 20

2.4 Yield-strength anomaly in superalloys . . . . . . . . . . . . . . . 21

2.5 The split-Hopkinson bar . . . . . . . . . . . . . . . . 27

2.5.1 Pochhammer-Chree oscillations . . . . . . . . . . 29

3 A model for the yield-strength anomaly 33

3.1 Constitutive model . . . . . . . . . . . . . . . . . . . . 33

3.1 .1 Time discretization . . . . . . . . . . . . . 37

3.2 Cobalt-base superalloys . . . . . . . . . . . . . . . . . . . . . . 39 
3.2.1 Numerical simulations . . . . . . . . . . . . . . . 43

3.3 VascoMax C-250 . . . . . . . . . . . . . . . . . . . . 51

3.3.1 Numerical simulations . . . . . . . . . . . . 55

$3.4 \quad$ MAR-M247 . . . . . . . . . . . . . . . . . . . . . . 59

3.4.1 Experiments . . . . . . . . . . . . . 60

3.4.2 Numerical simulations . . . . . . . . . . . . . . . 68

4 CP modelling of MAR-M247 DS 73

4.1 Crystal plasticity model . . . . . . . . . . . . 73

4.1 .1 Time discretization . . . . . . . . . . . . 77

4.2 Finite-element modelling . . . . . . . . . . . . . . . . 78

4.3 Parameter identification . . . . . . . . . . . . . 83

4.4 Results . . . . . . . . . . . . . . . . . 85

5 Conclusions and Future Work $\quad 99$

5.1 Conclusions . . . . . . . . . . . . . . . . . . . . . . . 99

5.2 Future work . . . . . . . . . . . . . . . . 100

$\begin{array}{ll}\text { Appendix A Notes on continuum mechanics } & 103\end{array}$

A.1 Stresses and strains definitions . . . . . . . . . . . . . . . . 104

$\begin{array}{ll}\text { Appendix B Euler angles } & 105\end{array}$

$\begin{array}{lll}\text { Appendix C Mesh-size sensitivity } & 107\end{array}$

$\begin{array}{ll}\text { Appendix D Implicit dymamic analysis } & 111\end{array}$

$\begin{array}{ll}\text { References } & 117\end{array}$

$\begin{array}{ll}\text { List of tables } & 131\end{array}$

$\begin{array}{ll}\text { List of figures } & 133\end{array}$ 


\section{Agradecimientos}

En primer lugar, me gustaría empezar dando las gracias a las personas que han hecho posible la consecución de desarrollar y presentar esta tesis doctoral. A mi director, Francisco Gálvez, por haber depositado su confianza en mí y haberme dado la posibilidad de disfrutar de esta etapa académica, y también de mi vida, durante los últimos años. A Javier Segurado, sus consejos y ayuda han sido claves a la hora de afrontar esta tesis. A los dos, gracias por vuestra dedicación y apoyo.

En segundo lugar, me gustaría agradecer a todos los compañeros del Departamento de Ciencia de Materiales el cariño mostrado desde el primer momento. Ha sido muy gratificante haber compartido, y espero seguir haciéndolo en el futuro, vivencias e inquietudes profesionales con este maravilloso grupo. Particularmente, siento la necesidad de citar a ciertas personas. A Rosa, Ana y David por su labor en el buen funcionamiento del departamento. A Alberto, Jose Alberto, Andrés y Luis Mariano, ya que son esenciales en la tarea investigadora del departamento y un pedacito de esta tesis es gracias a ellos. A David Cendón, Carlos González, Álvaro Ridruejo y Mihaela Iordachescu, por haberme prestado su ayuda en diversos momentos. A Vicente, por darme la oportunidad de colaborar en proyectos internacionales. De forma especial a Dani, Sandra, Miguel, Maricely, Bea y Josemi, con quienes he mantenido una relación más estrecha dentro y fuera del departamento, sin olvidar a Víctor, ese compañero de despacho que se convierte en amigo y con el que terminas compartiendo grandes momentos y divertidas anécdotas. Y cómo no, a Gustavo Esteban por sus consejos y ánimos durante las partes más difíciles de este camino.

En tercer lugar, quiero expresar mi gratitud hacia la gente del Structural Impact Laboratory (SIMLab) de la Universidad de Ciencias y Tecnología de 
Noruega (NTNU) por haberme hecho sentir como en casa. Al profesor Magnus Langseth por haber aceptado mi solicitud y hacer posible la estancia. Y especialmente, a Chus y Borja, por toda la ayuda ofrecida antes, durante y después de la estancia. También debo dar las gracias al Consejo Social de la Universidad Politécnica de Madrid por haber financiado este periodo de investigación.

Finalmente, estas últimas líneas son para agradecer a mi familia, lo más importante, todo el apoyo mostrado. A mis padres y a mi hermana, por ser un pilar fundamental de mi vida y deberles el poder haber llegado hasta aquí. A Pili, Jose, Macu, Jose Fernando, Mundi, Esme, Javi, Santi, María, Luis, Nerea, Ángel y Alejandro, gracias por haberme dado tanto. 


\section{Abstract}

Thermo-mechanical fatigue and creep behaviour are two of the most studied topics when characterising the performance of materials used for jet engines components. However, there are situations, such as blade-off events or foreign object damage, in which impact loads are the main thread to the structural integrity.

An efficient design process of the engine components requires both an experimental campaign and numerical simulations. Numerical simulations are useful to analyse the influence of different parameters, such as impact conditions, thickness, materials, without performing expensive experimental tests. The prediction capabilities of the simulations rely on the accuracy of the material models. Nevertheless, the Johnson-Cook (JC) model, one of the most widely used for impulse loading, cannot describe the positive temperature dependence, also known as yield-strength anomaly (YSA), of some of the alloys that are present in jet engines (superalloys). Moreover, the heterogeneous grain structure of directionally solidified (DS) turbine blades results in a complex response that must be analysed through advance simulations techniques like the crystalplasticity finite-element method (CPFEM). This technique has been used by other researchers to investigate fatigue behaviour of DS structures; however, to the author's knowledge, no work has been found which analyses the mechanical response of DS superalloys at high strain rates using CPFEM.

In this thesis, a JC-type material model to describe the response of alloys with YSA under high strain rates or impact loading is developed. The model is suitable for computational purposes in the industry its implementation in commercial finite-element codes, such as Abaqus, is not tedious. The constitutive equation describes the flow-stress dependence on temperature through super- 
posing two terms with an empirical law. The first term accounts for the general response of the alloy (plastic-strain hardening and thermal softening) while the second term models the YSA region with a phenomenological law. The model is calibrated for three different alloys tested under high strain rates and different temperatures using the split-Hopkinson bar technique. The chosen alloys were a cobalt-base superalloy (Co-12Al-10W), a maraging steel (VascoMax C-250) and a directionally solidified nickel-based superalloy (MAR-M247). In all cases, the capability of the model to replicate the experimental dynamic flow behaviour at different temperatures is demonstrated.

Finally, the anisotropic flow stress behaviour of the MAR-M247 DS alloy is studied in detail through the CPFEM technique under quasi-static and dynamic loading. Virtual samples of the whole gauge length of the specimens are generated considering the grain structure and orientations of the DS superalloy. The elastic-visco-plastic response of each crystal is modelled with phenomenologicalbase equations but taking into account the dislocation-dislocation interaction among the different slip systems. The constants of the model are fitted with the information from tests parallel to the grain-growth direction and the simulation strategy predicts with high accuracy the experimental response in the perpendicular direction. The mechanical constants of the quasi-polycrystal and polycrystal version of the alloy are also predicted with accuracy. The simulations reveal that in oligocrytalline structures, such as the one presented by the alloy, the yield-strength value is controlled by the grains with higher Schmid factor, while this influence decreases when plastic strain increases. Moreover, the analysis of stress micro-fields confirms that perpendicular grains to the loading axis are prone to nucleate cavities due to the pronounced local-stress increase factor. 


\section{Abbreviations}

The following abbreviations are used in this thesis:

$\begin{array}{ll}\text { APB } & \text { Antiphase boundary (energy) } \\ \text { BCC or bcc } & \text { Body-centred cubic (lattice) } \\ \text { CPFEM } & \text { Crystal-plasticity-finite-element method } \\ \text { CRSS } & \text { Critical resolved shear stress } \\ \text { DS } & \text { Directionally solidified } \\ \text { EBSD } & \text { Electron backscatter diffraction } \\ \text { FCC or fcc } & \text { Face-centred cubic (lattice) } \\ \text { FE } & \text { Finite element } \\ \text { FEM } & \text { Finite-element method } \\ \text { FFT } & \text { Fast Fourier transform } \\ \text { HCP or hcp } & \text { Hexagonal close-packed (lattice) } \\ \text { K-W or KW } & \text { Kear-Wilsdorf (locking mechanism) } \\ \text { PDF } & \text { Probability density function } \\ \text { RVE } & \text { Representative volume element } \\ \text { SHPB } & \text { Split-Hopkinson pressure bar } \\ \text { SHTB } & \text { Split-Hopkinson tension bar } \\ \text { SX } & \text { Single crystal } \\ \text { TBC } & \text { Thermal-barrier coating } \\ \text { VPSC } & \text { Viscoplastic self-consistent (model) } \\ \text { YSA } & \text { Yield-strength anomaly }\end{array}$




\section{Notation}

The notation adopted throughout the thesis is detailed below.

\section{Variables}

$A_{b} \quad$ Cross-sectional area of the bars

$A_{s} \quad$ Cross-sectional area of the sample

$\sigma \quad$ One-dimensional true stress

$\bar{\sigma} \quad$ Equivalent stress. Von-Mises definition

$s \quad$ One-dimensional engineering stress

$\varepsilon \quad$ One-dimensional true strain

$\dot{\varepsilon}_{0} \quad$ Reference strain rate

$\varepsilon_{p} \quad$ One-dimensional true plastic strain

$\bar{\varepsilon}_{p} \quad$ Equivalent plastic strain.

$\varepsilon_{i}(t) \quad$ Strain signal of the incident wave

$\varepsilon_{r}(t) \quad$ Strain signal of the reflected wave

$\varepsilon_{t}(t) \quad$ Strain signal of the transmitted wave

$e \quad$ One-dimensional engineering strain

$t \quad$ Time

$\dot{e} \quad$ One-dimensional engineering strain rate

$\rho \quad$ Mass density

$\rho_{b} \quad$ Mass density of the bars

$c \quad$ Wave velocity for a certain wavelength

$c_{0} \quad$ One-dimensional elastic wave velocity

E Young's modulus

$\nu \quad$ Poisson's ratio 


\begin{tabular}{|c|c|}
\hline$\mu$ & Shear modulus \\
\hline$E_{b}$ & Young's modulus of the bars \\
\hline$F$ & Force \\
\hline$\sigma_{\text {pulse }}$ & Maximum stress of the pulse \\
\hline$v_{p}$ & Projectile velocity \\
\hline$t_{p}$ & Time duration of the pulse \\
\hline$L_{p}$ & Length of the projectile \\
\hline$l_{0}$ & Gauge length of the specimen \\
\hline$D$ & Cross-section diameter \\
\hline$D_{0}$ & Initial cross-section diameter \\
\hline$\Delta l$ & Displacement \\
\hline$T$ & Temperature \\
\hline$T_{0}$ & Initial temperature \\
\hline$\beta$ & Taylor-Quinney coefficient \\
\hline$C_{p}$ & Specific heat capacity \\
\hline$R$ & Radius \\
\hline$\Lambda$ & Wave length \\
\hline$g^{\alpha}$ & Critical resolved shear stress on the system $\alpha$ \\
\hline$\xi_{0}$ & Strength of the pinning obstacle (Coutiño-Ortiz YSA model) \\
\hline$A_{c}$ & Positive constant (Coutiño-Ortiz YSA model) \\
\hline$T_{c}$ & Critical temperature constant (Coutiño-Ortiz YSA model) \\
\hline$\rho_{\text {cross }, 0}$ & Initial cross-slip dislocation density (Coutiño-Ortiz YSA model) \\
\hline$H^{\alpha}$ & Cross-slip enthalpy \\
\hline$k_{B}$ & Boltzmann constant \\
\hline$Y$ & Yield-stress function \\
\hline$Y_{m}$ & Yield-stress function of the matrix ( $\gamma$ phase) \\
\hline$Y_{p}$ & Yield-stress function of the precipitates ( $\gamma^{\prime}$ phase $)$ \\
\hline$\Delta Y_{p}$ & Yield-stress increment due to K-W locks \\
\hline$Y_{p f o r}$ & Forest dislocations hardening \\
\hline$Y_{p 0}$ & Maximum strength of the cross-slip pinning mechanism \\
\hline$T_{p}$ & Flow-stress-peak temperature \\
\hline$w_{p}$ & Constant for the width of the bell-shaped peak (YSA model) \\
\hline$n$ & Exponent of the superposition law \\
\hline
\end{tabular}


A Yield strength

$Q_{i} \quad$ Constant of the Voce-type hardening law. $i=1,2$.

$C_{i} \quad$ Constant of the Voce-type hardening law. $i=1,2$.

$T_{m} \quad$ "Melting" temperature of the Johnson-Cook and proposed model

$T_{r} \quad$ Constant, with temperature units, of the Johnson-Cook and proposed model

$m_{t} \quad$ Thermal exponent of the Johnson-Cook and proposed model

$k \quad$ Strain-rate-sensitivity exponent

$f \quad$ Yield function

$\boldsymbol{\sigma} \quad$ Cauchy stress tensor

$\varepsilon \quad$ Strain tensor

$\varepsilon^{e} \quad$ Elastic part of the strain tensor

$\varepsilon^{p} \quad$ Plastic part of the strain tensor

$\bar{\sigma} \quad$ Equivalent or von Mises stress

$\bar{\varepsilon}_{p} \quad$ Equivalent plastic strain

$\mathbb{C} \quad$ Fourth-order elastic moduli tensor

$\tau^{\alpha} \quad$ Resolved shear stress on the slip system $\alpha$

$\mathbf{s}^{\alpha} \quad$ Unit vector in the slip direction of the slip system $\alpha$

$\mathbf{m}^{\alpha} \quad$ Unit vector normal to the slip plane of the slip system $\alpha$

D Rate of deformation tensor

$\mathbf{D}^{e} \quad$ Elastic part of the rate of deformation tensor

$\mathbf{D}^{p} \quad$ Plastic part of the rate of deformation tensor

$\dot{\boldsymbol{\sigma}} \quad$ Rate of Cauchy stress tensor

$\dot{\boldsymbol{\sigma}}^{\nabla} \quad$ Objective rate of Cauchy stress tensor

A Set of hardening thermodynamic forces

N $\quad$ Flow vector

$\gamma \quad$ Plastic multiplier (macroscopic plasticity) or plastic-slip magnitude (crystal plasticity)

$\Psi \quad$ Plastic potential

$\boldsymbol{\alpha} \quad$ Set of internal hardening variables (macroscopic plasticity)

H Generalised hardening modulus

F Deformation gradient

$\mathbf{F}^{e} \quad$ Elastic part of the deformation gradient 
$\mathbf{F}^{p} \quad$ Plastic part of the deformation gradient

$\overline{\mathbf{F}} \quad$ Macroscopic deformation gradient

I Second-order identity tensor

$\mathbf{L}^{p} \quad$ Plastic part of the velocity gradient

q Set of internal variables (crystal plasticity)

S Second Piola-Kirchhoff stress tensor

$\mathbf{E}^{e} \quad$ Green-Lagrange strain tensor

$\mathbf{Q}^{\alpha \beta} \quad$ Strength-interaction matrix

$h(\Gamma) \quad$ Hardening law

$\Gamma \quad$ Accumulated plastic slip

$\mathbf{R} \quad$ Residual of a non-linear equation set.

$\mathrm{Q}^{\theta} \quad$ Transformation matrix

\section{Operations and symbols}

$\begin{array}{ll}a & \text { Scalar } \\ \mathbf{a} & \text { Vector, components } a_{i} \\ \boldsymbol{\alpha}, \mathbf{A} & \text { Second-order tensor, components } \alpha_{i j}, A_{i j} \\ \mathbb{A} & \text { Fourth-order tensor, components } A_{i j k l} \\ \mathbf{a} \cdot \mathbf{b} & \text { Scalar product of two vectors, } a_{i} b_{i} \\ \mathbf{a} \times \mathbf{b} & \text { Vectorial product } \\ \mathbf{a} \otimes \mathbf{b} & \text { Tensor product of two vectors, } a_{i} b_{j}\end{array}$

A : B Double inner dot product of tensors (double contraction), $A_{i j} B_{i j}$

$\mathbf{A} \cdot \mathbf{B} ; \mathbf{A B}$ Inner dot product of tensors (single contraction), $A_{i j} B_{j k}$

$\mathbb{A}: \boldsymbol{\alpha} \quad$ Inner product of a fourth and second order tensor $A_{i j k l} \alpha_{k l}$

$\begin{array}{ll}(\bullet) & \text { Material time derivative of }(\bullet) \\ (\bullet)^{-1} & \text { Inverse of }(\bullet) \\ (\bullet)^{\prime} & \text { Deviatoric part }(\bullet) \\ (\bullet)^{H} & \text { Hydrostatic part }(\bullet) \\ \operatorname{sign}(\bullet) & \text { The signum function }(\bullet) \\ \Delta(\bullet) & \text { Increment of }(\bullet) \\ \Delta_{x}(\bullet) & \text { Spatial gradient of }(\bullet)\end{array}$


Cінатren 1

\section{Introduction and Objectives}

\subsection{Introduction}

The flight of the Wright Flyer in 1903, the first successful heavier-than-air aircraft, marked the beginning of the aerospace industry. This industry, which has made the dream of flight comes true, has brought a positive impact on society by enhancing mobility and developing or improving materials and technologies that have been applied across many disciplines. For example, progress in aerodynamics and related facilities such as wind tunnels have been used both in auto racing and automotive industry. Satellite navigation system, enhanced radar technology, creation of standards for testing materials and development of modern non-destructive testing methods, such as structures with integrated sensors, have also been promoted by aerospace manufacturing [1, 2].

Likewise, the world of materials has undergone significant progress that is still ongoing. In 1930, wood parts of wings and fuselage started to be replaced by rolled aluminium alloys. This trend continued, introducing AA 2XXX, 6XXX and 7XXX aerospace aluminium alloys, into the 1970s, when polymer-matrix composites began to be used in military aircrafts seeking components be as light as possible yet be capable of withstanding high loads. However, the first generation of commercial airplanes with large amounts of composite parts $(>50 \%)$ was introduced in 2010 approximately [3].

Apart from the wings and fuselage, the aero engine is a key component of 
aircrafts since it is responsible for generating the power of the propulsion system. Nowadays, the jet engine is the common type in the industry and its operation is based on taking air and accelerating it to get a fast-moving jet that gives the thrust to the aircraft. Figure 1.1 shows the basic configuration of a jet engine. The engine sucks in air through the shaft and enters the compressor, which pressurizes the air continuously before reaching the combustion chamber. To that end, the compressor is made up of several stages with fixed vanes (stator) and rotating blades (rotor) attached to the shaft. In the combustion chamber, the air is sprayed with propellant and burned with the aim of increasing its temperature yet minimising the loss of pressure. Consequently, the stream of burning gases expands and accelerates through the turbine and nozzle, providing the high-velocity jet that thrusts the aircraft. Like on the compressor, the turbine if formed by rotating blades and fixed vanes split into different stages. On their way out, the gases move the turbine blades attached to the shaft, which spinning is used to drive the compressor and produce energy.

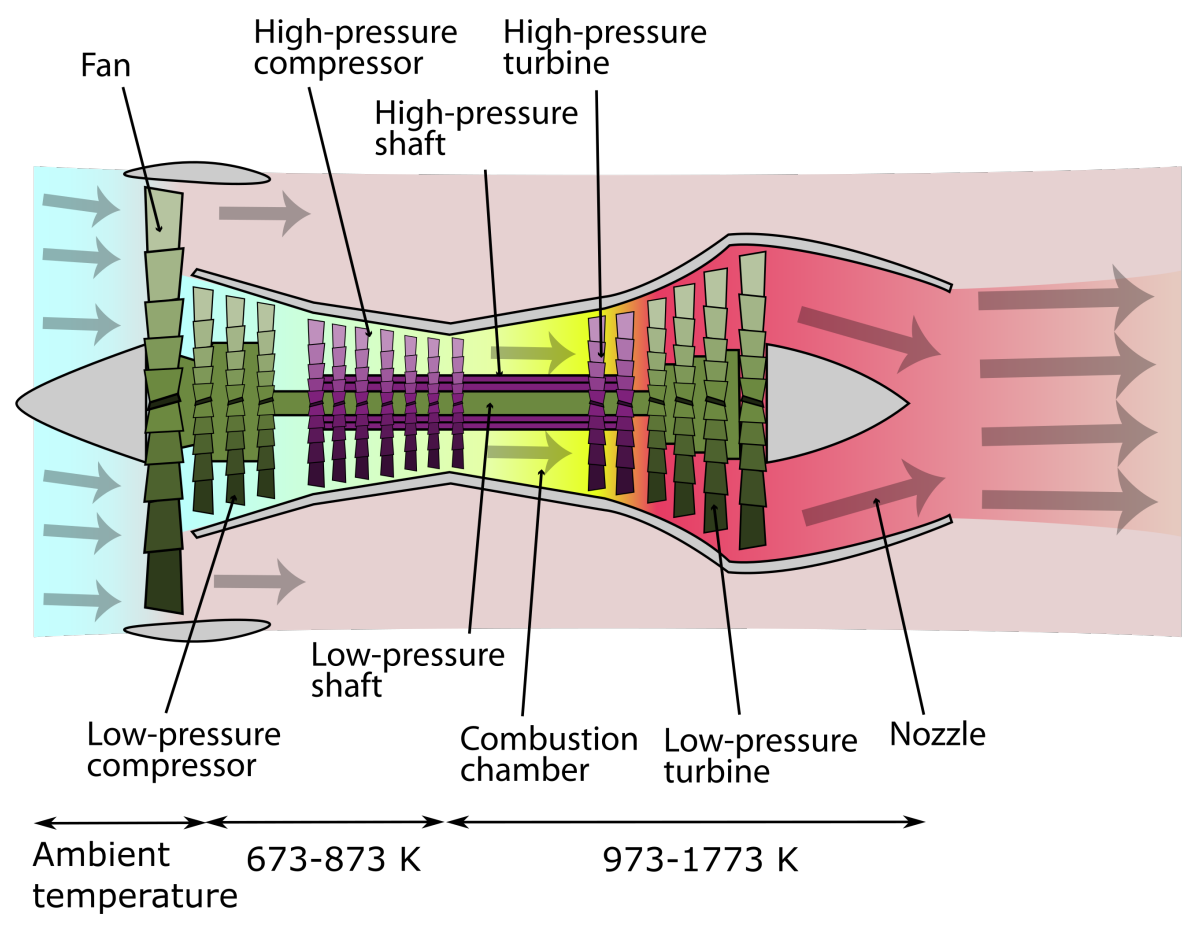

Figure 1.1: Illustration of the basic components of a jet engine. Adapted from [4]. 


\subsection{Introduction}

The materials that are used for the different parts of the jet engine tend to be as light as possible, but the selection is limited by the working temperature [5]. In the fan section, where heat restriction is not important, materials with high specific strength are required to withstand the centrifugal forces. Titanium and aluminium alloys are often used although carbon-fibre reinforced polymers are also an alternative. The fan case is also manufactured with aluminium or composites. In the compressor section, the working temperature increases up to temperatures between $773 \mathrm{~K}-873 \mathrm{~K}$ as air is pressurised. Due to the high temperature, materials such as aluminium alloys and carbon-fibre reinforced polymers must be disregarded and titanium alloys, such as Ti-6Al-4V, are the best choice. In addition, the last stages of the compressor may be made of nickel-base superalloys to increase the service time if temperatures above $823 \mathrm{~K}$ are achieved.

The efficiency of the engine is related to the temperature in the inlet of the turbine, so that the higher the temperature the higher the efficiency. This restriction, combined with the need for good yield strength and creep resistance, implies that cobalt- and, primarily, nickel-base superalloys are used for combustion chamber and turbine components. The yield strength of these materials (see Figure 1.2) does not decrease continuously with increasing temperature but remains constant or even increases during some temperature ranges. This behaviour is known as yield-strength anomaly (YSA). Figure 1.3 shows the evolution of the capabilities of nickel-base superalloys. For last decades, the performance of these alloys has been continuously improved not only by adjusting the amount of alloying elements and the conditions of the heat treatment but also by changing the processing technique to get directionally solidified (DS) and single crystal (SX) components that increase the creep resistance by removing the grain boundaries. Furthermore, the development of air-cooled blades and thermal-barrier coatings (TBC) have become critical to keep increasing the working temperature of the alloys. Nowadays, these technological advances have allowed the $1673 \mathrm{~K}\left(1400{ }^{\circ} \mathrm{C}\right)$ working temperature to be exceeded. 


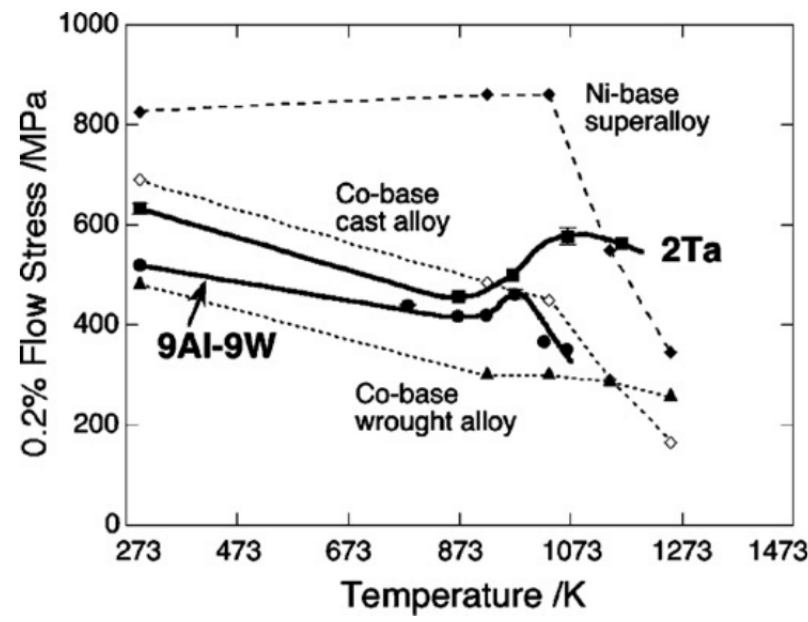

Figure 1.2: Examples of yield-strength anomaly (YSA) in Ni- and Co-base (9Al-9W, 2Ta) superalloys. Obtained from [6].

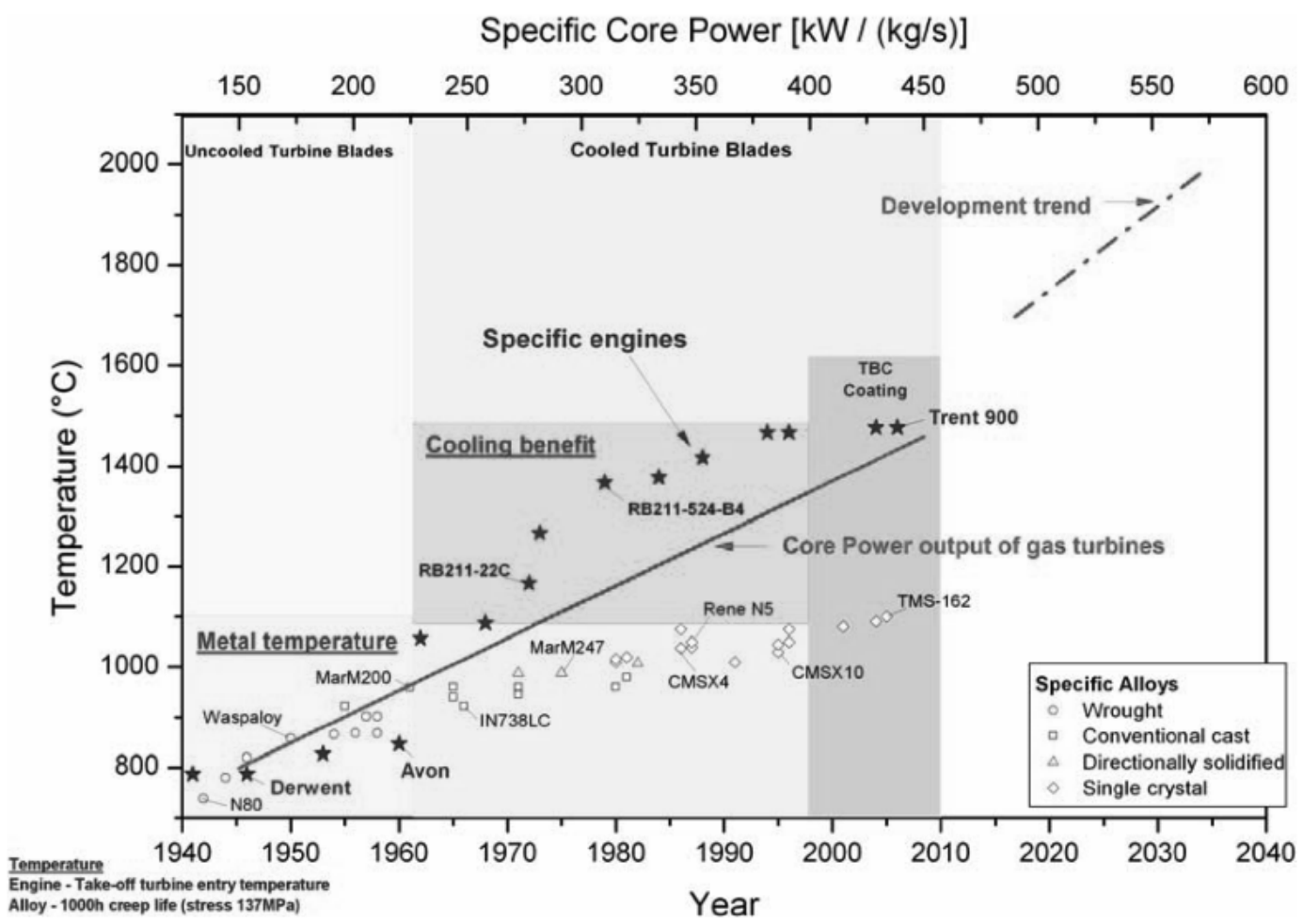

Figure 1.3: Evolution of the working temperature of nickel-base superalloys over the last decades [7]. 


\subsection{Motivation and objectives}

The common loads experienced by the components of a jet engine are thermomechanical fatigue and creep. However, there are some exceptions in which impact loads also occur, such as foreign object damage [8, 9] and blade-off events [10]. Foreign object damage takes place when foreign objects, such as sand or metallic debris, are sucked into the engine and impact onto different components. If these objects are small enough, they may reach the turbine section and damage the turbine blades and vanes. Conversely, blade-off events occur when a blade fails while rotating and impacts against the casing or other parts of the engine. In this case, the aero-engine standards require all fragments to be contained inside the casing.

Using large experimental campaigns to analyse the influence of different parameters (impact conditions, thickness, material, etc.) lead to an expensive design of the engine components. However, numerical simulations can help to cope with this issue. The prediction capabilities and therefore, the success of numerical simulations, depends on the accuracy of the material models. In the world of dynamic loading, defined as structures subjected to high-strainrate loading such as impact or blast loads, the Johnson-Cook model (JC) [11] is widely used for large-scale simulations because is simple and provide good predictions. Its constitutive equation describes the evolution of the stress by decomposing the effect of plastic strain, strain rate and temperature multiplicatively into three independent terms. The model works well with most of the metallic alloys and has been modified by several authors to account for the influence of the third deviatoric stress invariant in complex fracture patterns [12], the thermal softening of materials with phase transformation [13] and the anisotropic response of steel sheets [14], among others. Nevertheless, the model still fails with alloys that exhibit yield strength anomaly (YSA) and some authors, recently, have had to employ different model constants depending on the temperature range to simulate the ballistic performance of Ni-base superalloy plates [15].

Additionally, the complex response of heterogeneous structures like directionally solidified alloys makes it necessary to employ other strategies. Differ- 
ent approaches have been used to model the constitutive response of heterogeneous structures of this type, going from transversally isotropic viscoplastic models $[16,17]$, through the use of self-consistent schemes [18, 19], to the crystal-plasticity finite-element method (CPFEM) [20]. Transversally isotropic models are of interest when the computational cost is an issue, i.e., simulating a full structural component, but present three main drawbacks: (1) the assumption of one plane of isotropy is not true for a small numbers of grains in the cross-section, (2) strain and stress-fields' concentrations among grains are not replicated and (3) mechanical tests in at least three different directions are needed to fit the constants. These disadvantages can be overcome by representing explicitly the oligocrystalline microstructure and with the use of crystal plasticity (CP) since it takes into account the main deformation mechanisms, the shape and orientation of each grain. In previous studies, CPFEM models have been used to investigate the fatigue behaviour of DS Ni-base alloys [20], the stress and strain fields close to a crack tip [21] or the strain-field localisation in oligocrystalline specimens with the aim of analysing fatigue-crack nucleation [22]. Focusing the attention on the dynamic loading, only few studies can be found which model this regime. However, these studies are focused on standard polycrystalline microstructures and rely on more simple models, such as visco-plastic self-consistent models (VPSC) [23], or are applied to other types of alloys, such as titanium alloys [24, 25]. No work has been found, to the authors knowledge, which analyses the mechanical response of any DS superalloy at high strain rates using CPFE models.

Consequently, the thesis pursues two main objectives:

- Development of a Johnson-Cook-type material model that takes into account the yield-strength anomaly (YSA). To simulate the mechanical behaviour, under dynamic loading, of alloys characterised by an increase of strength with temperature (YSA). Accurate constitutive equations at high strain rates and for different temperature ranges are required, due to adiabatic heating, to obtain good numerical predictions of impact phenomena. The widespread of the Johnson-Cook (JC) model in the world of dynamic loading was the reason why it was chosen as a basis for the new 
model. The proposed model is applied to describe the response of three alloys tested at high strain rates, using the split-Hopkinson bar technique. The selected materials are a novel cobalt-base superalloy, a maraging steel and a DS nickel-base superalloy.

- Analysis of the deformation behaviour of directionally solidified (DS) structures under different strain rates by means of crystal-plasticity finiteelement method (CPFEM) and its prediction capabilities. To exploit the advantages of this technique with respect to macroscopic approaches or mean field methods when applying to heterogeneous structures with few grains and to assess its performance with dynamic implicit analysis. The CPFEM allows the researchers to accurately model the macroscopic response of a sample with a reduced number of parameters by considering the main deformation modes, texture and grain morphology and to predict the behaviour under a different loading scenario or microstructure configuration. Additionally, the simulation provides information about field distribution within the grains, which can be used to understand the microscopic deformation mechanisms and study the effect of microstructure in the failure of the material. The CPFEM approach is applied to a DS nickel-base superalloy. To that end, an algorithm was developed to get an explicit representation of the DS microstructure along the virtualspecimen gauge length.

Finally, the outline of the thesis is the following. The state of the art is contained within Chapter 2. It covers several topics: plastic deformation in metallic materials, macroscopic and crystal plasticity approaches, the JohnsonCook model, the physics-based explanation of the YSA with a review of the main strategies to model it and the split-Hopkinson bar technique. Chapter 3 presents the proposed JC-type model for materials that exhibit YSA. It also contains the dynamic behaviour of the cobalt-base superalloy, the maraging steel and the DS nickel-base superalloy at different temperatures and the calibration of the proposed model for each material. Lastly, the deformation behaviour of the DS nickel-base superalloy is studied in more detail through CPFEM in Chapter 4. 


\section{Снаттев 2}

\section{State of the art}

This chapter begins with an introduction to plastic deformation in metallic materials by dislocation glide. Then, in section 2.2, some basic lines of the mathematical theory of computational plasticity in the continuum framework are provided for both macroscopic and crystal plasticity approaches. Section 2.3 presents the Johnson-Cook constitutive model, which is widely used for impact modelling. A physics-based explanation of the yield-strength anomaly is given in section 2.4 together with a review of the main strategies to model it. Finally, in section 2.5, the Hopkinson-bar technique for testing at high strain rates is explained.

\subsection{Introduction to plastic deformation}

When a material is subjected to an external load, its shape changes; or in other words, the material deforms. If the change in shape is recoverable after the load is removed, it is called elastic deformation and is based on the relative change of the inter-atomic distances while keeping the bonding relationships among atoms. However, if the applied load is large enough, the material is not able to support it merely by elastic deformation and a permanent shape change occurs. This permanent shape change is called plastic deformation. In 
crystalline materials ${ }^{1}$, such as metals, plastic deformation mostly takes place by slip of planes of atoms along each other [26]. However, the theoretical shear stress required to plastically deform a crystal is much higher than the experimental value. This is because crystallographic planes do not move as blocks but taking advantage of dislocations (Orowan, Polanyi and Taylor, 1934).

Dislocations are line defects around which the crystal atoms do not occupy the equilibrium positions. Under a certain level of stress, the dislocations start to move through the crystal along the slip plane, causing the glide of one crystallographic plane at a time and the final crystal slip. The direction and magnitude of the slip is given by the Burgers vector of the dislocation. A schematic representation of the motion of an edge dislocation can be seen in Figure 2.1

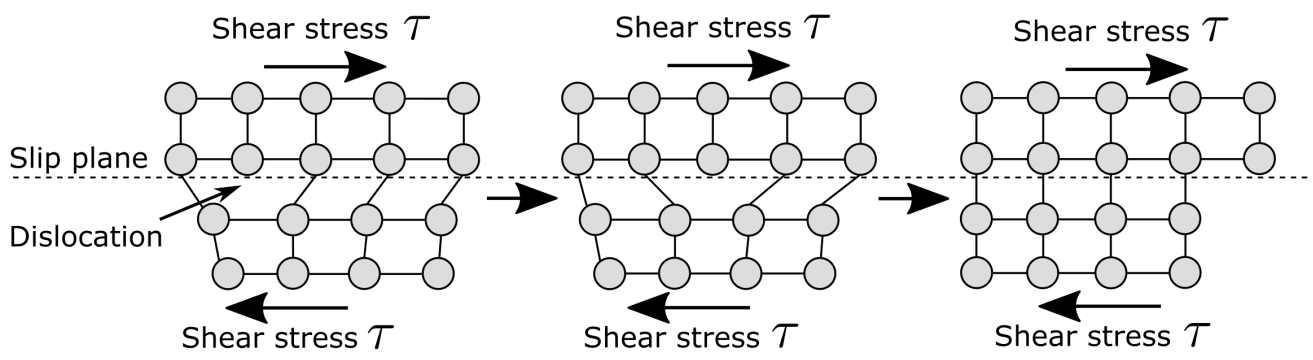

Figure 2.1: Motion of an edge dislocation in a crystal. The edge dislocation can be imagined as an extra half-plane of atoms [26].

Slip always takes place on a specific set of planes and along certain directions. As a consequence, plastic deformation occurs when the shear stress resolved in a slip system, which consists of a slip plane and a slip direction within that plane, reaches a critical value called critical resolved shear stress (Schmid's law). The resolved shear stress $\tau$ is the projection of the remote applied stress $\sigma$ on the slip plane and along the slip direction (Figure 2.2). In the simple case of uniaxial tension, the resolved shear stress is calculated as:

$$
\tau=\sigma \cos \phi \cos \lambda=M \sigma
$$

\footnotetext{
${ }^{1}$ Crystalline materials are solids in which atoms are positioned in order, forming a crystal lattice that extends in the three-dimensional space. The smallest repetitive portion is called unit cell. Face-centred cubic (fcc), body-centred cubic (bcc) and hexagonal close-packed (hcp) lattices are the most common metallic crystal structure.
} 
where $\phi$ is the angle between the applied-stress direction and the slip-plane normal, $\lambda$ the angle between the applied-stress and the slip direction and $M$ the Schmid factor. More generally, equation 2.1 reads:

$$
\tau^{\alpha}=\boldsymbol{\sigma}:\left(\mathbf{s}^{\alpha} \otimes \mathbf{m}^{\alpha}\right)
$$

where $\mathbf{s}$ is a unit vector in the slip direction, $\mathbf{m}$ the normal to the slip plane and $\alpha$ stands for the corresponding slip system. The available slip systems $\alpha=1,2,3 \ldots, N$ depends on the lattice of the crystal structure. For example, a material with a face-centred cubic lattice (fcc) has 12 slip systems defined by the family of $\{111\}$ planes and $<110>$ directions (four different set of planes and three different directions per plane set).

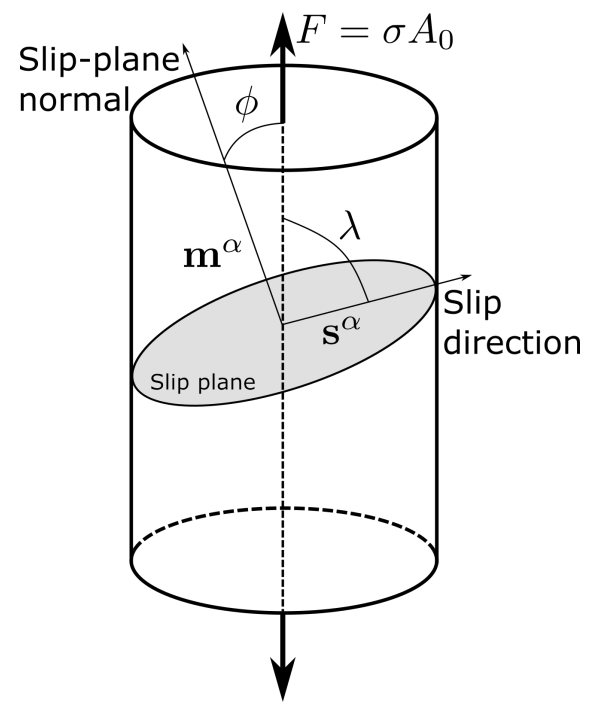

Figure 2.2: Relation between the stress applied in an uniaxial tensile test and the resolved shear stress acting in the slip system.

Besides the resolved shear stress, dislocation glide is also governed by temperature and strain rate. During their motion, dislocations interact with obstacles such as solute atoms, precipitates, other dislocations, grain boundaries, etc. Such obstacles represent an energy barrier (glide resistance) that must be overcome to allow dislocation motion. The energy barrier, as can be seen in Figure 2.3a, can be overcome by a combination of thermal and mechanical contributions. This means that the external force required to move dislocations decreases as temperature increases. However, in structural materials, there 
are always long-range obstacles (coarse precipitates, grain boundaries) that imply the need of an external applied stress to move dislocations, leading to an athermal stress component of the glide resistance (Figure 2.3b). In addition, deformation at high strain rates involves that dislocations must glide faster to accommodate the imposed displacement, decreasing the probability of getting enough thermal energy and hence, demanding a higher external stress.

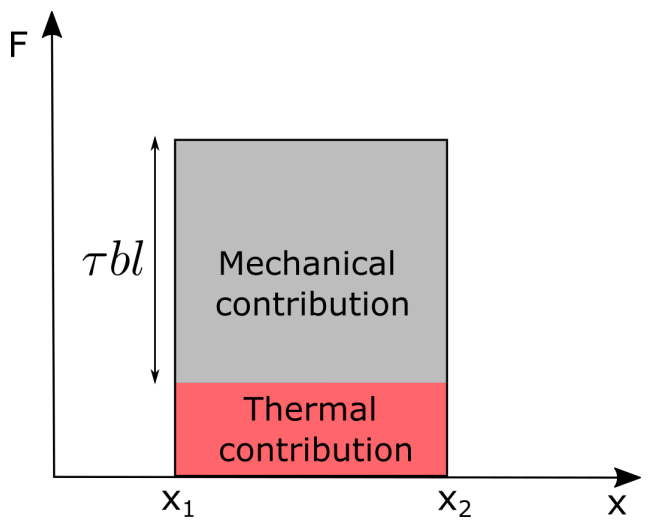

(a)

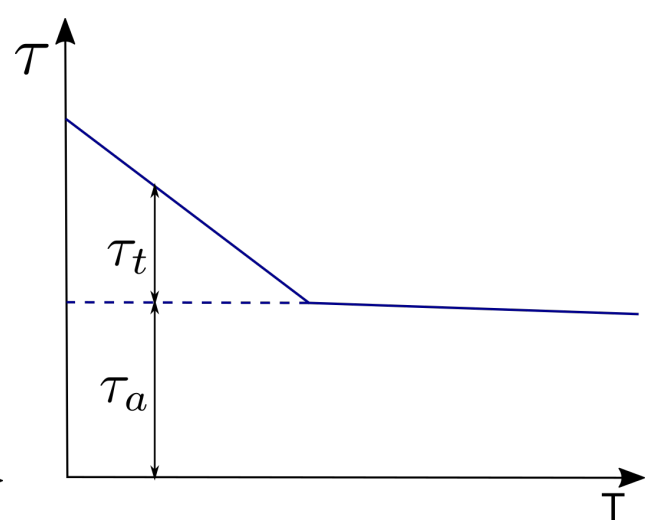

(b)

Figure 2.3: Representation of (a) the energy barrier that a dislocation, with length $l$, has to overcome to move from point $x_{1}$ to $x_{2}$ and (b) evolution of the macroscopically measured glide resistance as a function of temperature. The subscripts $a$ and $t$ stands for athermal and thermal components.

Finally, it is worth mentioning that above lines are just an introduction to plastic deformation by dislocation slip, the most common mechanism. For more information about other plastic-deformation mechanisms and fundamental principles of dislocation theory, the books written by William D. Callister [26] and D. Hull and D.J. Bacon [27] are recommended.

\subsection{Computational plasticity in the continuum framework}

The goal of this section is to give some basic lines of the mathematical theory of continuum models used to describe the hypoelasto-plastic behaviour of metallic materials. Detailed information about this topic can be found in [28] 
for macroscopic plasticity and in [29] for crystal plasticity. Basic notes on continuum mechanics can also be found in Appendix A.

\subsubsection{Macroscopic plasticity}

The rate of deformation tensor $\mathbf{D}$ can be decomposed additively [30] into the elastic $\mathbf{D}^{e}$ and plastic $\mathbf{D}^{p}$ part according to:

$$
\mathbf{D}=\mathbf{D}^{e}+\mathbf{D}^{p}
$$

The objective rate of the Cauchy stress tensor $\dot{\boldsymbol{\sigma}}^{\nabla}$ is thus defined using the elastic constitutive equation:

$$
\dot{\boldsymbol{\sigma}}^{\nabla}=\mathbb{C}^{\nabla}: \mathbf{D}^{e}=\mathbb{C}^{\nabla}:\left(\mathbf{D}-\mathbf{D}^{p}\right)
$$

where $\mathbb{C}^{\nabla}$ is the elastic stiffness tensor (fourth rank tensor) considering the corresponding rigid body rotations. If the material is isotropic and linear elastic, $\mathbb{C}^{\nabla}$ can be written as $\mathbb{C}$ and is given by:

$$
\mathbb{C}=\frac{\nu E}{(1+\nu)(1-2 \nu)} \mathbf{I} \otimes \mathbf{I}+\frac{E}{(1+\nu)} \mathbf{I}
$$

where $E$ is the Young's modulus of the material and $\nu$ the Poisson's ratio.

Plastic flow occurs when:

$$
f(\boldsymbol{\sigma}, \boldsymbol{A})=0
$$

where $f$ is the yield function (scalar) and is a function of the Cauchy stress tensor and a set of hardening thermodynamic forces $\boldsymbol{A}$. The von-Mises yield criterion [31] is an example of a widely used yield function for isotropic plasticity. In the simple and common case of isotropic hardening, $\boldsymbol{A}$ is equal to the hardening function of the material $Y\left(\bar{\varepsilon}_{p}\right)$, being the equivalent plastic strain $\bar{\varepsilon}_{p}$ a hardening variable $\boldsymbol{\alpha}$ of the model. The evolution of the plastic part of the strain tensor, also known as flow rule, is given by:

$$
\mathbf{D}^{p}=\dot{\gamma} \mathbf{N}=\dot{\gamma} \frac{\partial \Psi(\boldsymbol{\sigma}, \mathbf{A})}{\partial \boldsymbol{\sigma}}
$$


being $\mathbf{N}$ the flow vector, that defines the direction in which plastic flow takes place, and $\dot{\gamma}$ the time derivative of the so-called plastic multiplier $\gamma . \Psi(\boldsymbol{\sigma}, \boldsymbol{A})$ is called plastic potential and is equal to the yield function $f(\boldsymbol{\sigma}, \boldsymbol{A})$ in the case of associative plasticity. Associative plasticity is habitual for describing metallicalloys behaviour. Besides the flow rule, a hardening law is also required to complete the characterization of plasticity. The set of hardening variables $\boldsymbol{\alpha}$, that updates the set of hardening thermodynamical forces $\boldsymbol{A}$, evolves as:

$$
\dot{\boldsymbol{\alpha}}=\dot{\gamma} \mathbf{H}=-\dot{\gamma} \frac{\partial \Psi(\boldsymbol{\sigma}, \mathbf{A})}{\partial \boldsymbol{A}}
$$

The loading and unloading conditions may be defined as:

$$
\dot{\gamma} \geq 0 ; \quad f \leq 0 ; \quad \dot{\gamma} f=0
$$

being $\dot{\gamma} \geq 0$ interpreted as plastic loading, $f \leq 0$ as elastic loading and $\dot{\gamma} f=0$ as yielding. To determine the plastic multiplier $\gamma$, the next additional condition (consistency condition) is required:

$$
\dot{f}=0
$$

\subsubsection{Crystal plasticity}

Crystal plasticity is formulated assuming the multiplicative decomposition of the deformation gradient $\mathbf{F}$ into an elastic $\mathbf{F}^{e}$ and a plastic $\mathbf{F}^{p}$ component [32]:

$$
\mathbf{F}=\mathbf{F}^{e} \mathbf{F}^{p}
$$

where $\mathbf{F}^{p}$ defines the state of a virtual intermediate configuration that is unstressed and with the same coordinate system as the reference configuration. Considering the slip theory of plastic deformation, which has been introduced in Section 2.1, $\mathbf{F}^{p}$ can be identified as a measure of the slide of the crystal structure along crystallographic systems, while $\mathbf{F}^{e}$ accounts for the elastic distortion and rigid-body rotations of the crystal lattice (Figure 2.4). 


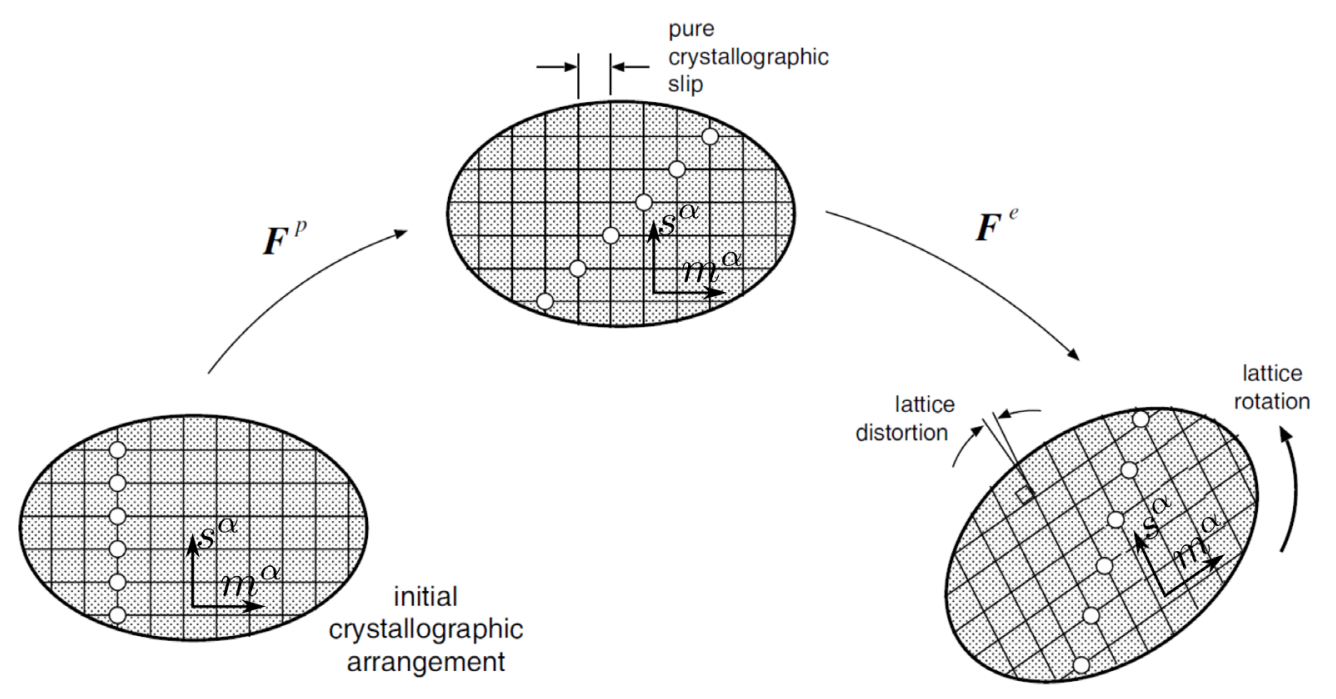

Figure 2.4: Micromechanical basis of the multiplicative decomposition of the deformation gradient. Adapted from [28].

Hence, the plastic deformation gradient of a crystal with $N$ different slip systems $\alpha$ is defined as:

$$
\mathbf{F}^{p}=\mathbf{I}+\sum_{\alpha=1}^{N} \gamma^{\alpha}\left(\mathbf{s}^{\alpha} \otimes \mathbf{m}^{\alpha}\right)
$$

where $\gamma$ represents the magnitude of plastic slip and $\mathbf{s}$ and $\mathbf{m}$ are, respectively, the unit vectors in the slip direction and normal to the slip plane. The evolution of the plastic deformation gradient can be calculated as a function of the velocity gradient $\mathbf{L}^{p}$ :

$$
\dot{\mathbf{F}}^{p}=\mathbf{L}^{p} \mathbf{F}^{p}
$$

The velocity gradient $\mathbf{L}^{p}$ is nothing but a measure of the rate of plastic deformation in the crystal, so it can be written as a sum of all slip rates from the different slip systems [33]:

$$
\dot{\mathbf{L}}^{p}=\sum_{\alpha=1}^{N} \dot{\gamma}^{\alpha}\left(\mathbf{s}^{\alpha} \otimes \mathbf{m}^{\alpha}\right)
$$

The evolution of plastic slip while loading is defined through the constitutive equations. Usually, it is assumed that the evolution of plastic slip depends on 
the resolved shear stress $\tau^{\alpha}$ and a set of internal variables $\mathbf{q}$, such as the critical resolved shear stress, chosen to describe the state of the crystal:

$$
\dot{\gamma}^{\alpha}=\dot{\gamma}^{\alpha}\left(\tau^{\alpha}, \mathbf{q}\right)
$$

Depending on the functions employed to describe the evolution of $\gamma^{\alpha}$ and $\mathbf{q}$ during deformation, the CP model can be classified as phenomenological or physics-based model.

The resolved shear stress $\tau^{\alpha}$ is calculated as:

$$
\tau^{\alpha}=\mathbf{S}:\left(\mathbf{s}^{\alpha} \otimes \mathbf{m}^{\alpha}\right)
$$

where $\mathbf{S}$ is the second Piola-Kirchhoff stress tensor, which reports the stresses in the reference configuration. Finally, $\mathbf{S}$ is defined by using the elastic response of the material:

$$
\mathbf{S}=\mathbb{C}: \mathbf{E}^{e}
$$

where $\mathbb{C}$ is the elastic stiffness tensor and $\mathbf{E}^{e}$ the Green-Lagrange strain tensor (intermediate configuration).

\subsubsection{Polycrystal homogenization}

Most of metallic materials used in engineering applications are polycrystalline structures, i.e., a solid made up of small crystals $(\sim \mu \mathrm{m})$, called grains, with random crystallographic orientations and different shapes and sizes. In such a case, unlike for single crystals and oligocrystals, determining the macroscopic response of the material by using crystal plasticity requires applying polycrystal homogenization (Figure 2.5).

Polycrystal homogenization is an approach to obtain the effective or macroscopic response of a polycrystal from the microscopic fields (stresses, strains, etc.) within the grains. Homogenization takes for granted that the characteristic length of the microscopic heterogeneities (grains) are much smaller than the macroscopic length scale but large enough to use continuum mechanics. Hence, it is assumed that the macroscopic fields $\bar{G}$ can be defined by averaging the 


\subsection{Computational plasticity in the continuum framework}

microscopic fields $G(\mathbf{x})$ over a volume $\omega$, usually called representative volume element RVE, that contains enough information about the microstructure:

$$
\bar{G}=\frac{1}{V} \int_{\omega} G(\mathbf{x}) d V=\frac{1}{V} \sum_{i} \int_{\omega_{i}} G(\mathbf{x}) d V_{i}
$$

being $\omega_{i}$ the different subdomains that form the volume $\omega$ and that represent the different grains or phases. Depending on the strategy followed to calculate the fields in each subdomain, there are two different homogenization schemes: the mean-field approach and the full-field approach [29].

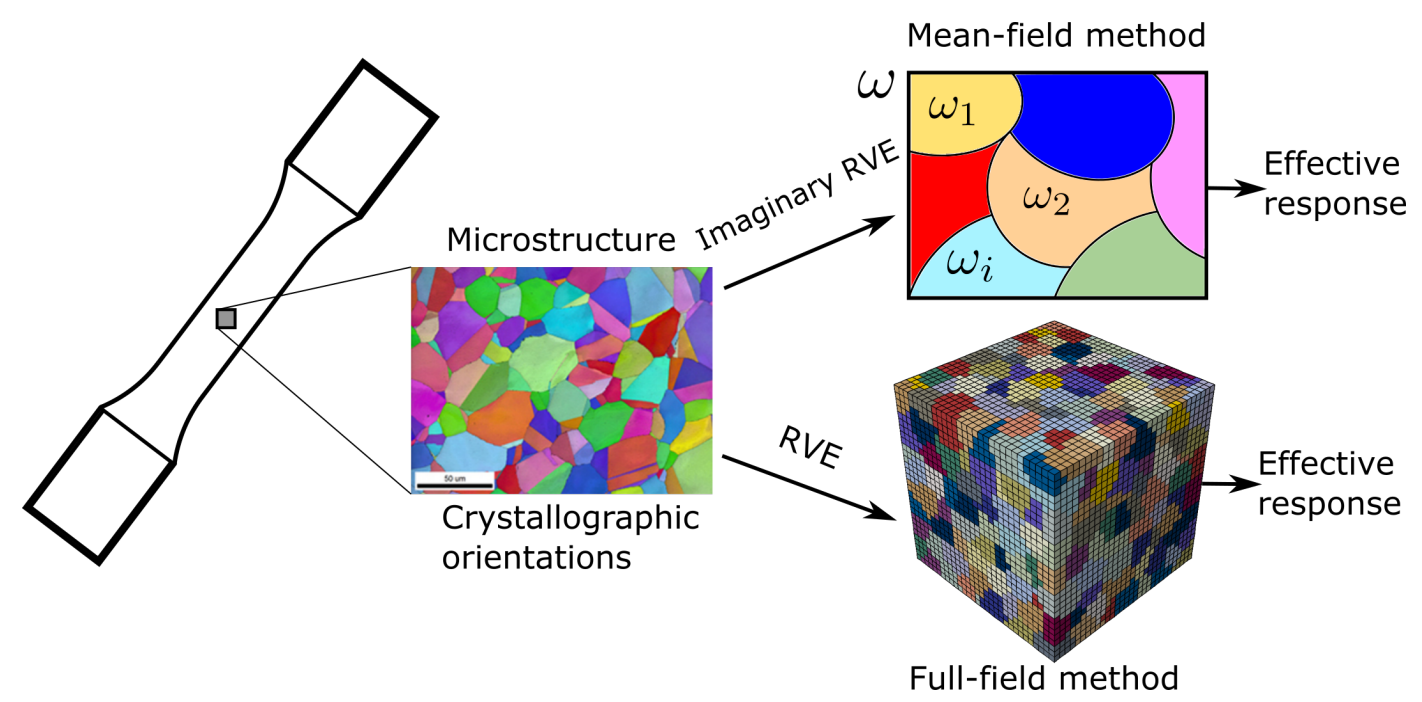

Figure 2.5: Different approaches for polycrystal homogenization.

On the one hand, all mean-field formulations share the assumption that the fields within the subdomains are constant values, being equal to the average value in the grain, but differ in the way in which the field interactions among the different subdomains are defined. For example, Taylor [34] assumed that all grains are subjected to the same amount of strain, that is equal to the macroscopic one, while Sachs [35] supposed an isostress condition. More realistic approaches were developed based on the Eshelby's solution [36] and on particular linearisation schemes, in which each grain is considered as an ellipsoid inside a homogeneous media, which properties are given by the average of the rest of the microstructure. This methodology, known as viscoplastic selfconsistent (VPSC) approximation, gives good predictions of both mechanical 
properties and texture evolution. In this context, it is important to mention the work of Lebensohn and Tomé [37] for presenting and implementing one of the most widely used VPSC approaches. Nevertheless, despite the good prediction capabilities (texture evolution, effective mechanical properties, deformed shape, etc [38]) of VPSC approximation through considering the interaction between each grain with its surroundings, it has, as any mean-field formulation, two main drawbacks: 1) the effect of local heterogeneities are not taken into account since grain shapes and orientations are described using average values and 2) the microfields are assumed to be constant within the grains. Although local heterogeneities and microfields are not so important to obtain the effective macroscopic response of a polycrystal, they are not negligible when dealing with damage or localized deformation [29].

On the other hand, full-field homogenization is characterised by predicting the polycrystal behaviour through finding the full-field solution of the mechanical response of a representative volume element (RVE) under homogeneous boundary conditions. The boundary value problem can be solved by using the finite element (FE) or the fast Fourier transform (FFT) method. This homogenization technique overcomes the VPSC shortcomings although the computational cost is higher. Most of the computational cost arises from the size of the RVE, but it should be large enough to supply an accurate statistical description of the microstructure [39]. The finite-element homogenization technique, which is used in this work, is discussed in more detail below, focussing attention on the required boundary conditions.

\section{FE computational homogenization}

The finite-element computational homogenization technique seeks to obtain the macroscopic response of a polycrystal from the crystal behaviour by solving the boundary value problem of a representative volume element (RVE) of the microstructure with the finite element method (FEM). In this approach, periodic boundary conditions should be applied to the RVE because the homogenized response is closer to the accurate solution than that obtained if displacement or forces are imposed [39]. The periodic boundary conditions connect the local 
displacements $(\mathbf{u})$ of the nodes on opposite faces with the far-field macroscopic deformation gradient $(\overline{\mathbf{F}})$ according to:

$$
\begin{aligned}
& \mathbf{u}\left(x_{1}, x_{2}, 0\right)-\mathbf{u}\left(x_{1}, x_{2}, L\right)=(\overline{\mathbf{F}}-\mathbf{I}) \mathbf{L}_{3} \\
& \mathbf{u}\left(x_{1}, 0, x_{3}\right)-\mathbf{u}\left(x_{1}, L, x_{3}\right)=(\overline{\mathbf{F}}-\mathbf{I}) \mathbf{L}_{2} \\
& \mathbf{u}\left(0, x_{2}, x_{3}\right)-\mathbf{u}\left(L, x_{2}, x_{3}\right)=(\overline{\mathbf{F}}-\mathbf{I}) \mathbf{L}_{1}
\end{aligned}
$$

where $L$ is the length of the cubic RVE, $\mathbf{L}_{i}=L \mathbf{e}_{i}$ and $x_{1}, x_{2}, x_{3}$ the coordinates of a point in the RVE system defined by the orthogonal unit vectors $\mathbf{e}_{1}, \mathbf{e}_{2}, \mathbf{e}_{3}$. The periodic boundary conditions in any FE software can be set by linking the displacement degrees of freedom of nodes on opposite cube faces with multipoint constraints. Defining a master node $M_{i}(i=1,2,3)$ for each of the three pairs of opposite faces, the far-field macroscopic deformation gradient $\overline{\mathbf{F}}$ can be applied to the RVE according to:

$$
\mathbf{u}\left(M_{i}\right)=(\overline{\mathbf{F}}-\mathbf{I}) \mathbf{L}_{i}
$$

where $\mathbf{u}\left(M_{i}\right)$ are the imposed displacements in the three master nodes. In case some components of the far-field macroscopic deformation gradient are not known, such as under uniaxial tensile loading, the boundary conditions are completed with the related components of the effective stresses by applying nodal forces $P$ to the master node $\left(M_{i}\right)$ and degree of freedom $(j)$ just as:

$$
P_{j}\left(M_{i}\right)=\left(\bar{\sigma} \mathbf{e}_{i}\right)_{j} A_{i}
$$

where $A_{i}$ is the area of the RVE face perpendicular to $\mathbf{e}_{i}$.

Finally, the polycrystal behaviour is calculated from the displacement and reaction forces of the master nodes. 


\subsection{The Johnson-Cook model}

This phenomenological model, presented by G.R Johnson and W.H Cook in [11], was formulated to describe the constitutive behaviour of metallic materials subjected to large strains, high strain rates and high temperatures; conditions that are achieved under impulse loadings such as high-velocity impacts. Although G.R Johnson and W.H Cook explained that other material models may give more accurate descriptions, they highlighted the suitability of the model for computational purposes due to its simplicity to be implemented in commercial codes.

The model assumes that the hardening function of any metallic material depends on the equivalent plastic strain $\bar{\varepsilon}_{p}$, equivalent plastic-strain rate $\dot{\bar{\varepsilon}}_{p}$ and temperature $T$ according to:

$$
Y\left(\bar{\varepsilon}_{p}, \dot{\bar{\varepsilon}}_{p}, T\right)=\left(A+B \bar{\varepsilon}_{p}^{n}\right)\left(1+C \ln \left(\frac{\dot{\bar{\varepsilon}}_{p}}{\dot{\varepsilon}_{0}}\right)\right)\left(1-\left(\frac{T-T_{r}}{T_{m}-T_{r}}\right)^{m_{t}}\right)
$$

where $\dot{\varepsilon}_{0}$ is a reference strain rate, $T_{r}$ the room temperature and $T_{m}$ the melting temperature. $A, B, n, C, m_{t}$ are the five constants of the model although $T_{r}$ and $T_{m}$ can also be considered constants of the model. The first term of the model $Y_{1}\left(\bar{\varepsilon}_{p}\right)$ accounts for the hardening during deformation, the second term $Y_{2}\left(\dot{\bar{\varepsilon}}_{p}\right)$ is responsible for the strain-rate sensitivity while the third term $Y_{3}(T)$ models the thermal softening.

Due to the widespread of the Johnson-Cook model in the world of dynamic loading, different authors have made modifications to fit their needs. Camacho and Ortiz [40] changed the original strain-rate-sensitivity term $Y_{2}\left(\dot{\bar{\varepsilon}}_{p}\right)$ to avoid undesired effects if $\frac{\dot{\bar{\varepsilon}}_{p}}{\dot{\varepsilon}_{0}}<1$ and proposed the use of a power law with the strainrate exponent $k$ :

$$
Y\left(\bar{\varepsilon}_{p}, \dot{\bar{\varepsilon}}_{p}, T\right)=Y_{1}\left(\bar{\varepsilon}_{p}\right)\left(1+\frac{\dot{\bar{\varepsilon}}_{p}}{\dot{\varepsilon}_{0}}\right)^{k} Y_{3}(T)
$$

T. Børvik et al. [41] adopted the modified strain-rate-sensitivity term, introduced a damage parameter coupled to the constitutive response and besides adapted the work-hardening part of the model $Y_{1}\left(\bar{\varepsilon}_{p}\right)$ by providing the possibility of using a Voce-type [42] hardening law, with new hardening constants 
$Q_{i}$ and $C_{i}$ (equation 2.24). In addition, S. Chocron et al. [12] added a new term $\mu_{\theta}$, called second-kind-Lode function, to consider the effect of the third invariant $J_{3}$ of the deviatoric stress tensor on the flow surface (equation 2.25).

$$
\begin{gathered}
Y\left(\bar{\varepsilon}_{p}, \dot{\bar{\varepsilon}}_{p}, T\right)=\left(A+\sum_{i=1}^{2} Q_{i}\left(1-\exp \left(-C_{i} \bar{\varepsilon}_{p}\right)\right)\right)\left(1+\frac{\dot{\bar{\varepsilon}}_{p}}{\dot{\varepsilon}_{0}}\right)^{k} Y_{3}(T) \\
Y\left(\bar{\varepsilon}_{p}, \dot{\bar{\varepsilon}}_{p}, T, J_{3}\right)=Y_{1}\left(\bar{\varepsilon}_{p}\right) Y_{2}\left(\dot{\bar{\varepsilon}}_{p}\right) Y_{3}(T) \mu_{\theta}\left(J_{3}\right)
\end{gathered}
$$

It is important to note that all variations of the original model keep the multiplicative decomposition of the flow stress to take into account the effect of plastic strain, strain rate and temperature; and in all cases, the temperature term is the same as the original power law $Y_{3}(T)$ and therefore, accounts only for thermal softening.

\subsection{Yield-strength anomaly in superalloys}

As stated in the introduction, nickel- and cobalt-based superalloys are used in the hottest parts of turbine engines, where temperatures above $800 \mathrm{~K}$ are achieved. The good performance of these alloys at high temperatures is grounded in the microstructure (Figure 2.6a), a face-centred cubic (fcc) matrix, called $\gamma$ phase, and precipitates with a $\mathrm{L}_{2}$ ordered structure, called $\gamma^{\prime}$ phase, based on intermetallic compounds such as $\mathrm{Ni}_{3}(\mathrm{Ti}, \mathrm{Al})$ or $\mathrm{Co}_{3}(\mathrm{Al}, \mathrm{W})$. The thermal softening of the matrix is offset against the positive temperature dependence of the precipitates strength, keeping almost constant the flow strength evolution of the alloy or even getting higher values between some temperatures [43, 44]. The structure of $\gamma^{\prime}$ phase, which lattice is depicted in Figure 2.6b, is similar to an fcc lattice but in which the atoms that occupy the corner sites are different from the atoms that occupy the face-centred sites ( $\mathrm{Al}$ and $\mathrm{Ni}$, respectively, for the $\mathrm{Ni}_{3} \mathrm{Al}$ compound).

Figure 2.7 shows the common flow strength behaviour of $\gamma^{\prime}$ precipitates. At low temperatures (region I), slip on octahedral planes $\{111\}$ is the dominant plastic deformation mechanism. However, in the $\mathrm{L}_{2}$ structure, the slip vector 


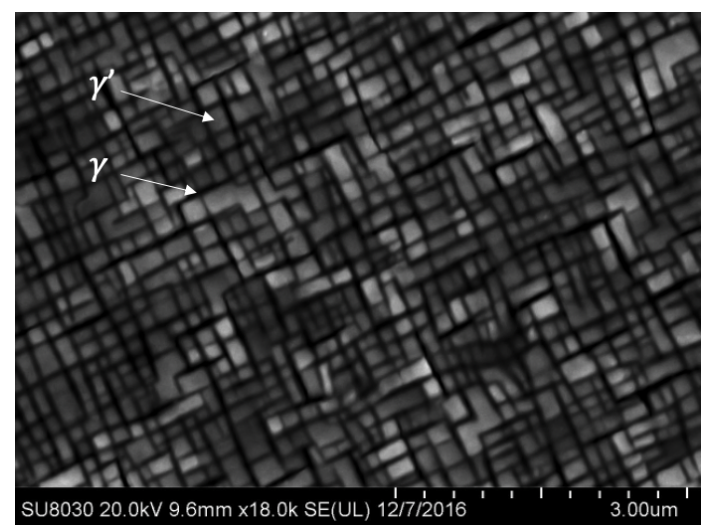

(a)

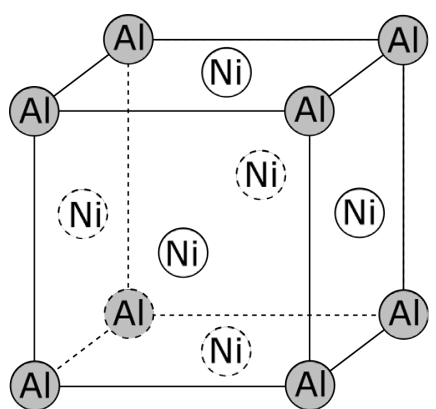

(b)

Figure 2.6: (a) Typical $\gamma / \gamma^{\prime}$ microstructure of superalloys with rectangular $\gamma^{\prime}$ precipitates and (b) $\mathrm{L}_{2}$-type unit cell of $\mathrm{Ni}_{3} \mathrm{Al}$ intermetallic compound.

$\frac{1}{2}<110>$ is not lattice translation, i.e., an $\mathrm{Al}$ atom moves and occupies the position of a Ni atom, and a surface disorder, with an associated energy called antiphase boundary energy (APB), is left behind when the dislocation glide. The order in the structure is restored again when another $\frac{1}{2}<110>$ dislocation passes in the same direction and on the same plane; so, in $\mathrm{L}_{2}$ structures, $\frac{1}{2}<110>$ dislocations tend to move by pairs to minimise the energy of the lattice. The movement, at the same time, of two $\frac{1}{2}<110>$ dislocations with an associated APB energy is usually called superdislocation. Since the APB energy associated with the cube $\{010\}$ planes is lower than the one associated with $\{111\}$ planes [45], it may occur that $\frac{1}{2}<110>$ screw dislocations on $\{111\}$ cross-slip onto $\{010\}$, getting stuck and becoming immobile dislocations (Kear-Wilsdorf (K-W) mechanism [46, 47]).

Figure 2.8 depicts in more detail the K-W locks. The $\frac{1}{2}<110>$ dislocations tend to cross-slip on cube planes to decrease the APB energy. Since the crossslip segments can only glide a small distance on the cube planes, the $\frac{1}{2}<110>$ superpartials $^{2}$ move again onto a parallel (111) or onto a non-parallel (11̄1) plane. At this point, the APB and the superpartials are no longer coplanar, and hence, dislocations become immobile [48]. Usually, $\frac{1}{2}<110>$ superpartials are dissociated into two $\frac{1}{2}<112>$ Shockley partials because it is energetically

\footnotetext{
${ }^{2}$ Superpartial term refers to any of the two $\frac{1}{2}<110>$ dislocations that form a superdislocation
} 


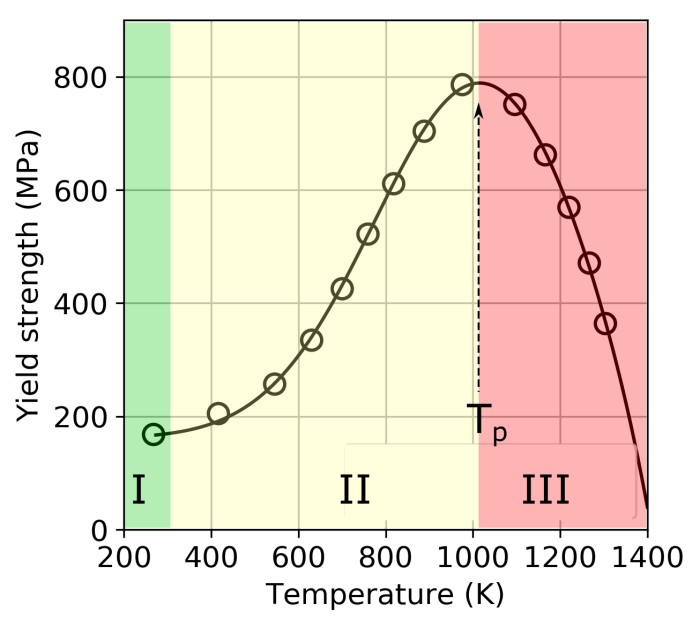

Figure 2.7: Variation of the $\mathrm{Ni}_{3} \mathrm{Al}$ yield strength with temperature. Data from [49].

favourable when gliding on $\{111\}$ planes. However, Shockley partials cannot cross-slip and they must recombine before leaving the current $\{111\}$ slip plane. The energy barrier for Shockley-partials constriction can be overcome with a mechanical $\left(\tau_{p e}\right)$ or a thermal contribution; being the reason why cube crossslip is a thermally activated process and therefore, the probability of K-W locks increases with temperature, pining more dislocations and getting a higher flow strength (region II).

Finally, this trend is broken above the peak temperature $T_{p}, 1000 \mathrm{~K}$ approximately, when dislocations glide on $\{010\}$ planes activates and a sudden decrease of the flow strength occurs (region III) [43].

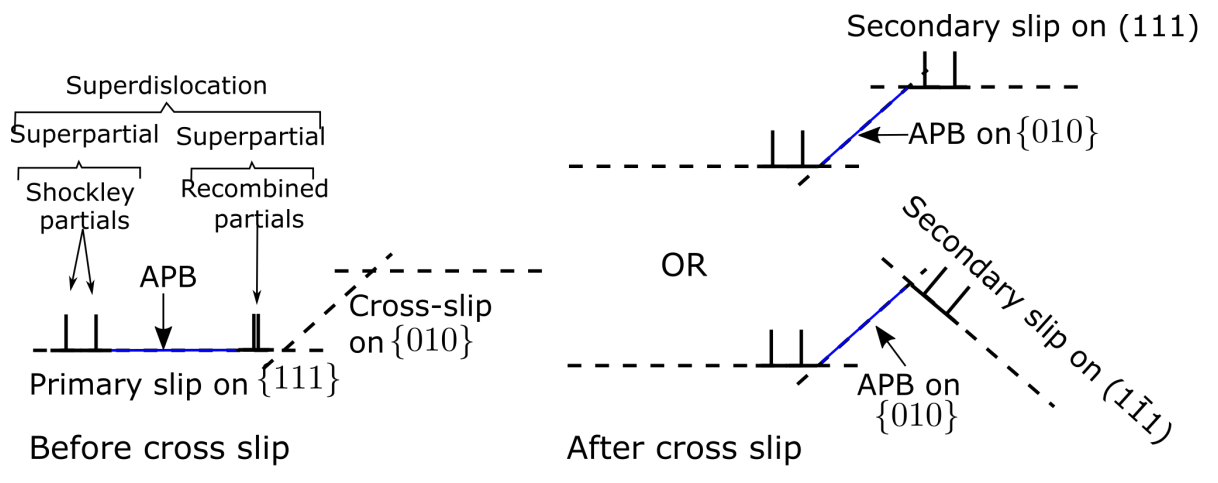

Figure 2.8: Kear-Wilsdorf locks. After cross-slipping, the APB and the dislocations are no longer coplanar, creating pinning points [48]. 
Several authors have proposed models to reproduce the anomalous temperature behaviour of $\mathrm{L}_{2}$ structures and Ni-base superalloys. Cuitiño and Ortiz [48] developed a dislocation-base model for $\mathrm{L}_{2}$ intermetallic crystals by assuming the combined effect of forest dislocations and K-W locks. Then, other authors $[50,51,52]$ used the basis of the Cuitiño-Ortiz model and proposed different approaches to describe the response of Ni-base superalloys, in which both $\gamma$ and $\gamma^{\prime}$ phases contribute to the hardening of the material. Essentially, in those models, the slip resistance $\left(g^{\alpha}\right)$ on the octahedral slip systems depends on the density of sessile dislocations due to cross-slip through the equation:

$$
g^{\alpha}=\mu \xi_{0} \exp \left(\frac{A_{c}}{T-T_{c}}\right) \sqrt{\rho_{\text {cross }, 0} \exp \left(-\frac{H^{\alpha}}{k_{B} T}\right)}
$$

where $\mu$ is the shear modulus, $T$ is the temperature, $\xi_{0}, A_{c}, T_{c}$ are constants that define the temperature dependence of the strength of the pinning obstacles, while the initial density of pinned dislocations $\rho_{\text {cross }, 0}$, the activation enthalpy for cross-slip $H^{\alpha}$ and the Boltzmann constant $k_{B}$ describe the $\mathrm{K}-\mathrm{W}$ locks (obstacles) density (cross-slip probability) at a given temperature and for a given enthalpy. So, the yield strength anomaly is modelled through the competition between the hardening due to the increase of cross-slip and the weakening of the obstacle strength as temperature increases. $H^{\alpha}$ is far from being a constant value but depends on crystallographic orientation according to [48]:

$$
H^{\alpha}=\frac{\mu b^{3}}{4 \pi}\left[h+k_{1}\left(\frac{b \tau_{p e}^{\alpha}}{\Gamma^{111}}-k_{2} \frac{b \tau_{s e}^{\alpha}}{\Gamma^{111}}\right)+\sqrt{\frac{2 \pi \Gamma^{111}}{\mu b}\left(\frac{1}{\sqrt{3}}-\frac{\Gamma^{010}}{\Gamma^{111}}+\frac{b\left|\tau_{c b}^{\alpha}\right|}{\Gamma^{111}}\right)}\right]
$$

being $h, k_{1}, k_{2}$ material constants, $\Gamma^{111}, \Gamma^{010}$ the APB energies per unit area on the octahedral and cubic planes and $\tau_{x x}^{\alpha}$ three non-Schmid shear stresses. These non-Schmid stresses promote or hinder the cross-slip mechanism. $\tau_{c b}$ implies that cross-slip is promoted by the resolved shear stress on cubic planes while $\tau_{p e}$ and $\tau_{s e}$ stand for the resolved shear stresses on the primary and secondary slip planes (see Figure 2.8) in the edge direction and that play an important role during the recombination of Shockley-partials before cube cross-slip. Depending on their sign, these stress components may help to merge the partials and 
promote cross-slip or the opposite, difficult Shockley-partials constriction and hence, decrease the probability of cross-slip. These non-Schmid stresses are responsible for the tension-compression asymmetry and Schmid's law violation in $\mathrm{L}_{2}$-type single crystals $[48,50,51,52]$.

Alternatively, other authors [53, 54] stated that the probability of K-Wlocks formation follows a normal distribution and therefore, it can be used to describe the yield stress peak of $\gamma^{\prime}$ (Figure 2.7). Hence, the pinning strength can be written as:

$$
\xi=\xi_{0} \exp \left(-\frac{\left(T-T_{p}\right)^{2}}{w_{p}^{2}}\right)
$$

where $\xi_{0}$ is the maximum stress increment due to cross-slip pinning, $w_{p}$ is a constant that defines the width of the bell-shaped region of the yield strength evolution and $T_{p}$ is the temperature at which the flow-stress peak appears (see Figure 2.7). In the normal distribution, unlike the equation 2.26, the loss of strength after the peak temperature is as a consequence of the dominant role of cube slip in the deformation mechanism.

Finally, the author of the present thesis presented in [55] a pure phenomenological way to model the yield-strength anomaly by following the strategy employed by B. Erice [13] to describe the softening behaviour of metallic materials with phase transformations. We proposed that the flow-strength evolution with temperature of any material that shows YSA can be modelled as:

$$
Y(T)=Y_{0} \Theta(T)
$$

In the above equation, $Y_{0}$ is the flow strength at room temperature and $\Theta(T)$ a piecewise function given by:

$$
\Theta(T)=\left\{\begin{array}{l}
\Theta_{1}(T) \text { if } T \leq T_{1} \\
\Theta_{2}(T) \text { if } T_{1}<T \leq T_{2} \\
\Theta_{3}(T) \text { if } T_{2}<T \leq T_{m}
\end{array}\right.
$$


where $T_{1}$ and $T_{2}$ define the temperature range in which the material shows a positive temperature dependence. The functions $\Theta_{1}(T), \Theta_{2}(T), \Theta_{3}(T)$ reads:

$$
\begin{gathered}
\Theta_{1}(T)=1 \pm\left[\frac{\Delta \sigma_{1}}{\sigma_{r}}\left(\frac{T-T_{r}}{T_{1}-T_{r}}\right)^{m_{t 1}}\right] \\
\Theta_{2}(T)=\Theta_{1}(T)\left[1 \pm\left[\frac{\Delta \sigma_{2}}{\sigma_{1}}\left(\frac{T-T_{1}}{T_{2}-T_{1}}\right)^{m_{t 2}}\right]\right] \\
\Theta_{3}(T)=\Theta_{2}(T)\left[1-\left(\frac{T-T_{2}}{T_{m}-T_{2}}\right)^{m_{t 3}}\right]
\end{gathered}
$$

where $m_{t 1}, m_{t 2}, m_{t 3}$ are material constants that controls the shape of the softening and/or hardening curve and $T_{m}, \sigma_{r}, \sigma_{1}, \Delta \sigma_{1}, \Delta \sigma_{1}$ are also constants defined according to Figure 2.9. It is important to note that the \pm sign, which set the positive or negative temperature dependence, is established by the sign of $\Delta \sigma$. The main drawback of this model is the large number of constants that must be fitted; being the reason why it was not used in the present work.

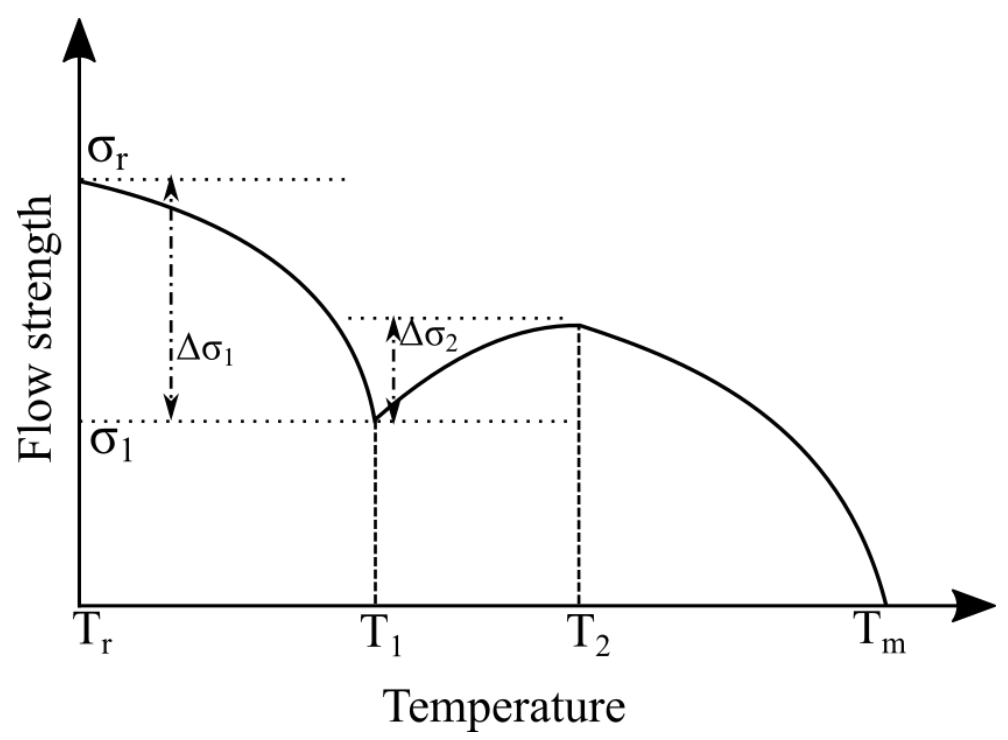

Figure 2.9: Schematic representation of the flow strength evolution with temperature in an alloy that exhibits YSA. 


\subsection{The split-Hopkinson bar}

The split-Hopkinson bar technique is used for investigating the mechanical response of materials at high strain rates, in the range of $10^{2}-10^{4} \mathrm{~s}^{-1}$. The basis of this technique comes from the experiment developed by B. Hopkinson in 1913 [56] to measure the pressure pulse produced by the impact of a bullet or an explosion at the end of a cylindrical rod. In 1949, H. Kolsky [57] added a second bar to the original Hopkinson device and studied the mechanical properties of specimens under compressive loading at high strain rates. Some years later, in 1960, J. Harding et al. [58] presented a version of the apparatus for tensile loading.

Essentially, the split-Hopkinson bar set-up (see Figure 2.10) consists of two homogeneous elastic bars, called incident (input) and transmission (output) bars, a striker bar (projectile) and the specimen, which is located between the two bars. The input and output bars must have a large length-to-diameter ratio, larger than 20 [59], to ensure $1 \mathrm{D}$ wave propagation. In the compressive configuration (Figure 2.10a), the projectile impacts, at velocity $v_{p}$, on the free end of the incident bar and generates a compressive-stress trapezoidal pulse. The maximum stress $\sigma_{p u l s e}$ and time duration $t_{p}$ of the pulse introduced in the system can be controlled through the length $L_{p}$ and velocity $v_{p}$ of the projectile, according to [60]:

$$
\begin{gathered}
\sigma_{\text {pulse }}=\rho_{b} c_{0} \frac{v_{p}}{2} \\
t_{p}=2 \frac{L_{p}}{c_{0}}
\end{gathered}
$$

where $\rho_{b}$ is the density, $c_{0}=\sqrt{E_{b} / \rho_{b}}$ is the elastic wave velocity and $E_{b}$ is the Young's modulus of the bars.

After the impact, the wave travels along the incident bar at a speed $c_{0}$ and when reaches the bar/specimen interface, it is partially transmitted to the output bar and partially reflected. The transmitted stress pulse has the same sign as the incident pulse, whereas it is the opposite for the reflected one. Assuming dynamic equilibrium and applying the theory of wave propagation in 1D elastic media [60] to a set-up made of bars with the same diameter and 
material (the most common case), the force $F(t)$ and the displacement $\Delta l(t)$ applied to the specimen can be calculated as:

$$
\begin{gathered}
F(t)=E_{b} A_{b} \varepsilon_{t}(t) \approx E_{b} A_{b}\left(\varepsilon_{i}(t)+\varepsilon_{r}(t)\right) \\
\Delta l(t)=-2 c_{0} \int_{0}^{t} \varepsilon_{r}(t) d t
\end{gathered}
$$

where $A_{b}$ is the cross-sectional area of the bars. $\varepsilon_{i}(t), \varepsilon_{r}(t)$ and $\varepsilon_{t}(t)$ stand, respectively, for the incident, reflected and transmitted strain signals measured in the bars with the strain gauges. Differentiating the equation 2.35 with respect to time and considering the gauge length of the specimen $l_{o}$, the strain rate of the experiment is:

$$
\dot{e}(t)=\frac{-2 c_{0}}{l_{0}} \varepsilon_{r}(t)
$$

The strain rate can be selected by adapting the magnitude of the reflected pulse, i.e., by adapting the velocity of the projectile $v_{p}$ (see equations 2.32 and 2.34). Finally, the stress $(s, \sigma)$ and strain $(e, \varepsilon)$ values can be computed as:

$$
\begin{gathered}
s(t)=\frac{F(t)}{A_{s}} ; e(t)=\frac{\Delta l}{l_{0}} \\
\sigma(t)=s(t)(1+e(t)) ; \varepsilon(t)=\ln (1+e(t))
\end{gathered}
$$

where $A_{s}$ is the initial cross-section area of the specimen, $s, e$ refer to the engineering definitions and $\sigma, \varepsilon$ to the true-values definition, assuming volume conservation and taking tension as positive and compression as negative.

It is important to note that split-Hopkinson bar tests, unlike quasi-static tests, are under adiabatic conditions due to its short time duration $(\sim 200 \mu \mathrm{s})$. This cause an increase of temperature in the specimen that can be estimated as [61]:

$$
T(t)=T_{0}+\frac{\beta}{\rho C_{p}} \int_{0}^{\varepsilon_{p}} \sigma d \varepsilon_{p}
$$

where $T_{0}$ stands for the initial temperature, $C_{p}$ is the specific heat capacity of the specimen, $\rho$ is its density and $\beta$ is the Taylor-Quinney coefficient, which 
represents the fraction of plastic work that is transformed into heat because it is not "stored" in the microstructure as defects (dislocations, vacancies, twins, etc.). The difference between isothermal and adiabatic deformation is that the heat that is generated does not have time to dissipate into the surroundings in adiabatic conditions and therefore, the temperatures increases.

In the tensile configuration, the bases of the experimental procedure are the same but for the method used for creating the stress pulse. Usually, the incident pulse is generated by launching, with compressed air, a tubular projectile against a flange connected to the end of the input bar (see Figure 2.10b). However, other methods can also be used for creating the tensile pulse [60].

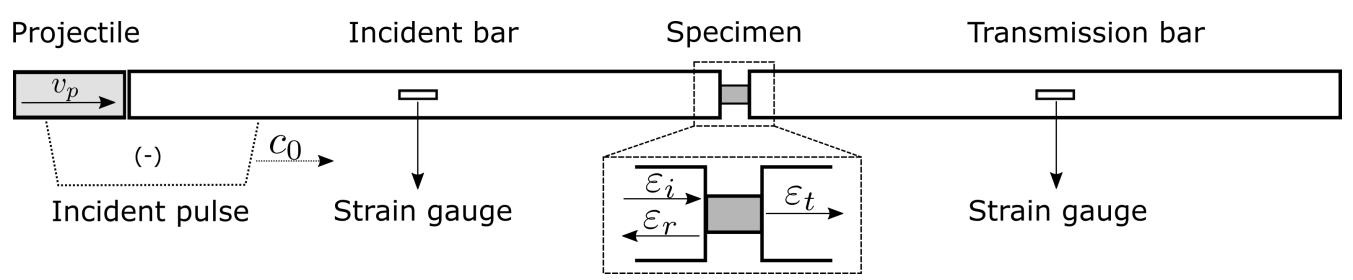

(a)

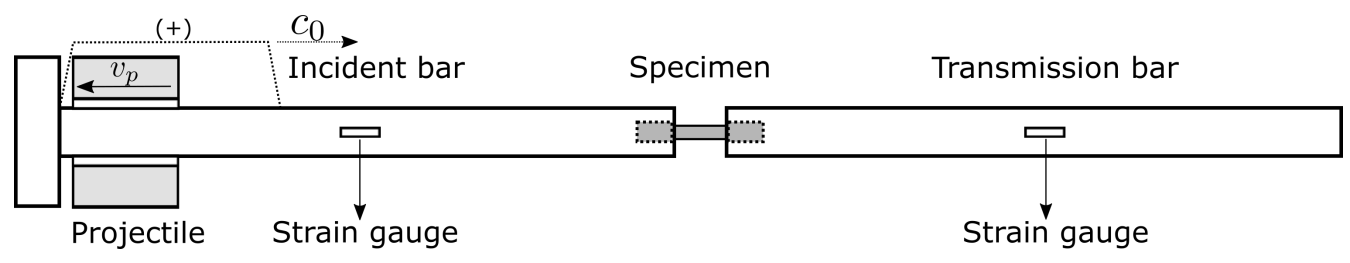

(b)

Figure 2.10: Scheme of the split-Hopkinson bar for the (a) compressive and (b) tensile configuration.

\subsubsection{Pochhammer-Chree oscillations}

The assumption that stress waves propagate in cylindrical bars with constant speed $c_{0}$ is not true. Davis [59] solved the equation of stress-wave propagation in an infinitely long cylindrical linear elastic bar (Pochhammer [62] and Chree [63]) and found that, in the case of the split-Hopkinson bar experiment, the speed of the stress pulse is not constant and depends on the wavelength (Figure 2.11a) of its constituent frequencies. The solution shows that the high frequency components (short wavelength) of the pulse propagate slower that 
the low frequency components (long wavelength), leading to the appearance of oscillations (Figure 2.11b).

Such oscillations, that appears both on the recorded signals from the strain gauges and on the final stress-strain curve, do not affect on the constitutive response of the material. However, they introduce some uncertainty when determining the yield strength in addition to the aesthetic aspect of the curve (Figure 2.12).

In the end, to attenuate the oscillations, different approaches may be applied:

- Dispersion correction by wave reconstruction. This methodology is grounded in transforming and reconstructing the strain gauge signals from their location to the specimen-bar interface by using Fourier series [64, 65].

- Pulse shapers. The dispersion of the elastic waves in the bars can be minimized by using a pulse shaper, a small piece of material that is placed between the projectile and the incident bar and that filters outer the highfrequency components of the pulse [60].

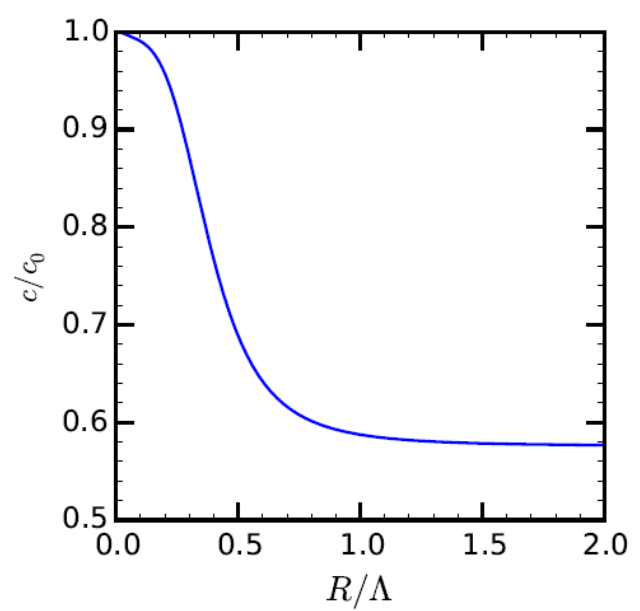

(a)

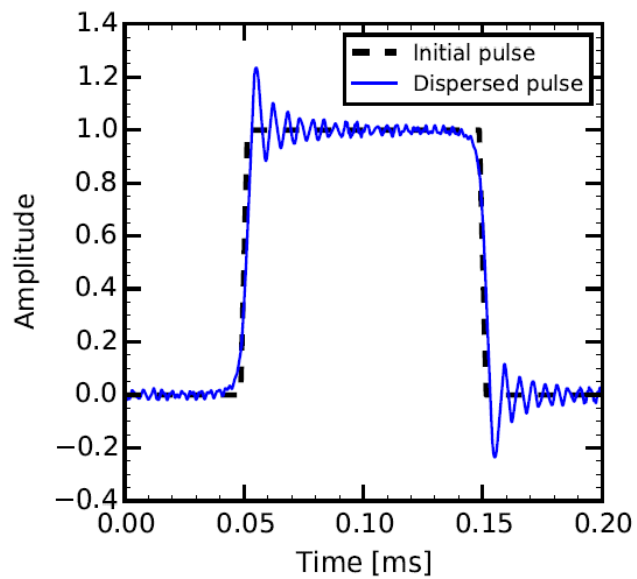

(b)

Figure 2.11: (a) Solution of the Pocchammer-Chree equation for a material with a Poisson's ratio $\nu=0.29$. The phase velocity is a function of the ratio between the bar radius $R$ and the wavelength $\Lambda$. (b) Dispersion of a trapezoidal pulse after travelling $1 \mathrm{~m}$ in a bar with $R=4.6 \mathrm{~mm}$ and $c_{0}=$ $5178 \mathrm{~m} / \mathrm{s}$. More details in [64]. 


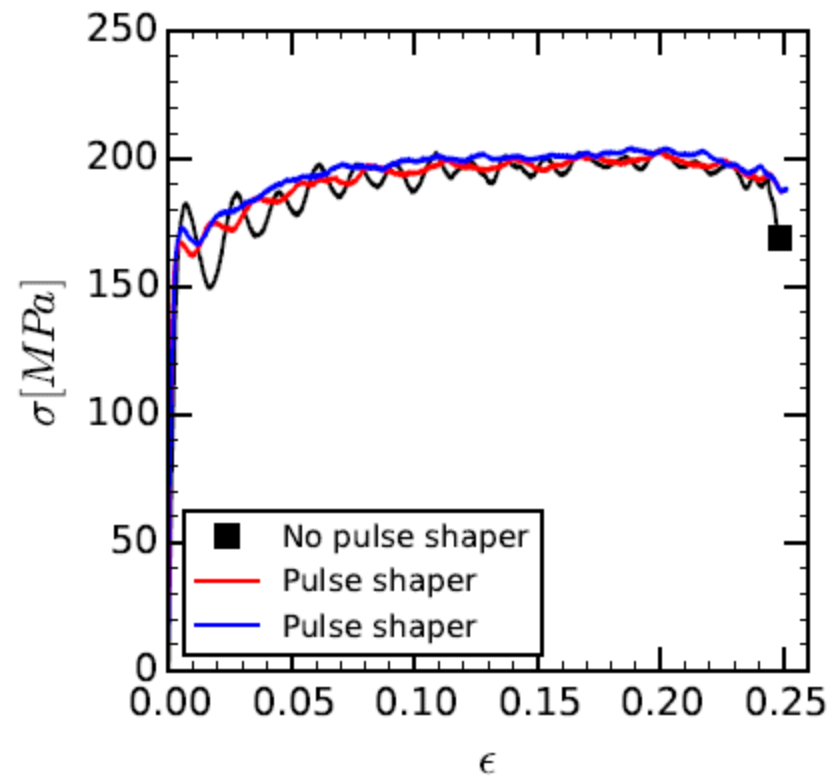

Figure 2.12: Comparison between the true stress-strain curves obtained from a SHPB experiment on aluminium with and without the use of a pulse shaper. 


\section{Chapter 3}

\section{A model for the yield-strength anomaly}

In this chapter, a material model that takes into account the yield-strength anomaly (YSA) and that can be used for ballistic applications is presented (section 3.1). The proposed model takes advantage of the multiplicative decomposition of the effect of plastic strain, temperature and strain rate on the hardening function (Johnson-Cook), while a normal-distribution-type equation is used to introduce the positive temperature dependence of the yield stress in the desired temperature range. Then, the proposed model is used to simulate the behaviour of three different alloys with YSA tested at high strain rates. The materials considered in this work were a cobalt-base superalloy (section 3.2), a maraging steel (section 3.3) and a nickel-base suparalloy (section 3.4). Although maraging steels do not present YSA per se, this kind of behaviour was achieved through precipitation hardening during the heating process in high-temperature tests.

\subsection{Constitutive model}

The model proposed in this work was formulated assuming hypoelasticity and isotropic behaviour. It was implemented in the Abaqus finite element 
code [66], through a VUMAT material subroutine, by following the elasticpredictor/return-mapping scheme detailed in [28].

Applying the small-strain theory ${ }^{1}$, the strain tensor $\varepsilon$ can be decomposed additively into its elastic $\varepsilon^{e}$ and plastic $\varepsilon^{p}$ part; being the elastic response of the material described as:

$$
\dot{\boldsymbol{\sigma}}=\mathbb{C}: \dot{\varepsilon}^{e}=\mathbb{C}:\left(\dot{\varepsilon}-\dot{\varepsilon}^{p}\right)
$$

where $\dot{\boldsymbol{\sigma}}$ is the rate of the Cauchy stress tensor and $\mathbb{C}$ the fourth-order stiffness tensor. Due to the material-isotropy assumption, $\mathbb{C}$ is completely denifed by the Young's modulus $E$ and Poisson's ratio $\nu$ of the material (see equation 2.5).

Employing the von Mises yield criterion [31], the yield function $f$ reads:

$$
f(\boldsymbol{\sigma}, Y)=\bar{\sigma}(\boldsymbol{\sigma})-Y\left(\bar{\varepsilon}^{p}, T, \dot{\bar{\varepsilon}}^{p}\right)
$$

being $\bar{\sigma}(\boldsymbol{\sigma})=\sqrt{3 / 2\left(\boldsymbol{\sigma}^{\prime}: \boldsymbol{\sigma}^{\prime}\right)}$ the von Mises or equivalent stress, $\boldsymbol{\sigma}^{\prime}$ the deviatoric part of the stress tensor and $Y\left(\bar{\varepsilon}^{p}, T, \dot{\bar{\varepsilon}}^{p}\right)$ a function, usually called hardening function, that defines the strength evolution of the material against the equivalent plastic deformation $\bar{\varepsilon}^{p}$, temperature $T$ and plastic-strain rate $\dot{\bar{\varepsilon}}^{p}$.

Using associative plasticity, the plastic strain tensor evolves according to (flow rule):

$$
\dot{\varepsilon}^{p}=\dot{\bar{\varepsilon}}_{p} \frac{\partial f}{\partial \boldsymbol{\sigma}}=\dot{\bar{\varepsilon}}_{p} \frac{3 \boldsymbol{\sigma}^{\prime}}{2 \bar{\sigma}}
$$

where the equivalent-plastic-strain rate $\dot{\bar{\varepsilon}}_{p}=\sqrt{2 / 3\left(\dot{\boldsymbol{\varepsilon}}^{p}: \dot{\boldsymbol{\varepsilon}}^{p}\right)}$ stands for the increase of plastic deformation accommodated by the material and $\partial f / \partial \boldsymbol{\sigma}$ represents the direction of plastic flow, which is normal to the yield surface in the present case.

The inelastic behaviour of superalloys is based on the contribution of the matrix ( $\gamma$ phase) and precipitates ( $\gamma^{\prime}$ phase). The matrix, FCC structure, behaves similarly to the majority of metallic alloys, i.e., hardening when increasing

\footnotetext{
${ }^{1}$ Since all tensor quantities are defined in a co-rotational coordinate system in a VUMAT material subroutine, small-strain theory is assumed. Therefore, the rate of deformation tensor $\mathbf{D}$ is equivalent to time derivative of the strain tensor $\dot{\varepsilon}$ and the objective rate of the Cauchy stress tensor $\dot{\boldsymbol{\sigma}}^{\nabla}$ to $\dot{\boldsymbol{\sigma}}$.
} 


\subsection{Constitutive model}

the plastic deformation and softening when increasing the temperature. Hence, the flow behaviour of the matrix can be written as:

$$
Y_{m}=\left(A+\sum_{i=1}^{2} Q_{i}\left(1-\exp \left(-C_{i} \bar{\varepsilon}_{p}\right)\right)\right)\left(1-\left(\frac{T-T_{r}}{T_{m}-T_{r}}\right)^{m_{t}}\right)
$$

where work hardening and thermal softening effects are decomposed multiplicatively. The strain hardening function is defined by a Voce-type equation [42], where $A$ stands for the yield strength of the material and $Q_{i}$ and $C_{i}$ are constants. The temperature dependence is considered through the power-law expression used in the Johnson-Cook model [11] (Section 2.3), where $T_{m}, T_{r}$ and $m_{t}$ are also constants.

In order to describe the flow stress of $\gamma^{\prime}$ phase, the constitutive expression shown in equation 3.5 is proposed. The flow-stress peak is modelled by using the normal distribution introduced in Section 2.4. The constant $Y_{p_{\text {for }}}$ stands for the resistance due to forest dislocations, while the right-side term of the equation takes into account the weakening of obstacles strength as temperature increases.

$$
Y_{p}=\left(Y_{p_{f o r}}+Y_{p 0} \exp \left(-\frac{\left(T-T_{p}\right)^{2}}{w_{p}^{2}}\right)\right)\left(1-\left(\frac{T-T_{r}}{T_{m}-T_{r}}\right)^{m_{t}}\right)
$$

The choice of using the normal distribution over the function presented by Cuitiño and Ortiz (equation 2.26 in Section 2.4) was made on the basis of: 1) ease for identifying the initial guess of the parameters and 2) flexibility for fitting data from different materials with YSA. Figure 3.1 shows the capability of the model to reproduce the yield-strength temperature dependence of $\mathrm{L}_{2}$ intermetallic compounds with significantly different behaviour. Figure 3.1a collects the data for the compound that hardens some Ni-base superalloys while Figure $3.1 \mathrm{~b}$ collects the information of Co-base-superalloys precipitates. The values of the constants appear in Table 3.1 and were obtained by using the non-linear least squares algorithm implemented in the function scipy.optimize.curve fit [67] for Python programming language. 


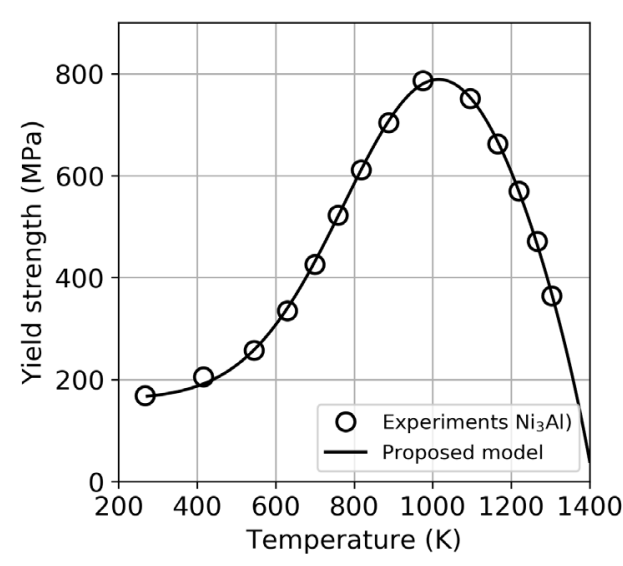

(a)

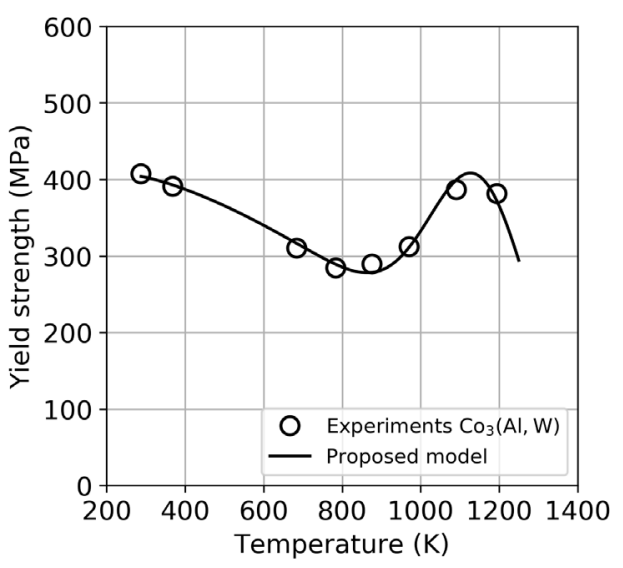

(b)

Figure 3.1: Yield strength evolution of the (a) $\mathrm{Ni}_{3} \mathrm{Al}$ compound with temperature in [001] loading direction [49] and (b) $\mathrm{Co}_{3}(\mathrm{Al}, \mathrm{W})$ compound [68].

Table 3.1: Values of the parameters that define the yield strength evolution of the $\mathrm{L}_{2}$ intermetallic compounds. The first row is for $\mathrm{Ni}_{3} \mathrm{Al}$ and the second row for $\mathrm{Co}_{3}(\mathrm{Al}, \mathrm{W})$

\begin{tabular}{ccccccc}
\hline$Y_{p_{\text {for }}}(\mathrm{MPa})$ & $Y_{p 0}(\mathrm{MPa})$ & $w_{p}(\mathrm{~K})$ & $T_{p}(\mathrm{~K})$ & $T_{m}(\mathrm{~K})$ & $T_{r}(\mathrm{~K})$ & $m_{t}(-)$ \\
\hline 161.0 & 629.1 & 346.2 & 1018.2 & 1409.3 & 265 & 15 \\
\hline 405.2 & 727.5 & 189.3 & 1200.0 & 1500.0 & 265 & 1.4
\end{tabular}

Using the empirical superposition law employed in [69, 70, 54], the strength of the YSA alloy can be set as:

$$
Y=\left(Y_{m}^{n}+Y_{p}^{n}\right)^{(1 / n)}
$$

where $1 \leq n \leq 2$ is an exponent that must be fitted.

Usually, we only have experimental data of the YSA alloy and not any information about the response of the matrix and/or the precipitates independently. In this situation, fitting all constants of the model is not worthy. Accordingly, the equation 3.5 can be simplified as:

$$
\Delta Y_{p}=Y_{p 0} \exp \left(-\frac{\left(T-T_{p}\right)^{2}}{w_{p}^{2}}\right)
$$




\subsection{Constitutive model}

in order to only model the region where the stress peak appears, while the general shape of the flow stress evolution is defined by $Y_{m}$.

Finally, the strain-rate sensitivity of the alloy was considered by using the power-law term presented by Camacho and Ortiz [40]. The final version of the model reads:

$$
Y\left(\bar{\varepsilon}_{p}, T, \dot{\bar{\varepsilon}}_{p}\right)=\left(Y_{m}\left(\bar{\varepsilon}_{p}, T\right)^{n}+\Delta Y_{p}(T)^{n}\right)^{(1 / n)}\left(1+\frac{\dot{\bar{\varepsilon}}_{p}}{\dot{\varepsilon}_{0}}\right)^{k}
$$

where $\dot{\bar{\varepsilon}}_{p}$ is the equivalent-plastic-strain rate, $\dot{\varepsilon}_{0}$ a reference strain rate and $k$ the strain-rate-sensitivity exponent.

It should be noted that the temperature $T$ is a variable that evolves according to equation 2.39 due to adiabatic heating during dynamic loading and repeated here, in the rate form, for completeness.

$$
\dot{T}(t)=\frac{\beta}{\rho C_{p}} \bar{\sigma} \dot{\bar{\varepsilon}}_{p}
$$

\subsubsection{Time discretization}

The non-linear finite-element problem is linearised through applying the boundary conditions as a function of time and discretising the total time with time increments $\Delta t$. Employing the backward-Euler scheme in the time interval $t_{n}-t_{n+1}$, the stress update consists in solving the next set of equations (3.10 3.15), knowing that the VUMAT subroutine gives us $\Delta_{t}, \varepsilon_{n}^{e}, \Delta \varepsilon, \boldsymbol{\sigma}_{n}, \bar{\varepsilon}_{p_{n}}, T_{n}$ with respect to a co-rotational coordinate system [66]:

$$
\begin{gathered}
\varepsilon_{n+1}^{e}=\varepsilon_{n}^{e}+\Delta \varepsilon-\Delta \bar{\varepsilon}_{p} \frac{3 \boldsymbol{\sigma}_{n+1}^{\prime}}{2 \bar{\sigma}_{n+1}} \\
\varepsilon_{n+1}^{p}=\varepsilon_{n}^{p}+\Delta \bar{\varepsilon}_{p} \frac{3 \boldsymbol{\sigma}_{n+1}^{\prime}}{2 \bar{\sigma}_{n+1}} \\
\bar{\varepsilon}_{p_{n+1}}=\bar{\varepsilon}_{p_{n}}+\Delta \bar{\varepsilon}_{p} \\
T_{n+1}=T_{n}+\frac{\beta}{\rho C_{p}} \boldsymbol{\sigma}_{n} \Delta \bar{\varepsilon}_{p}
\end{gathered}
$$




$$
\begin{gathered}
\boldsymbol{\sigma}_{n+1}=\mathbb{C}: \boldsymbol{\varepsilon}_{n+1}^{e} \\
\Delta \bar{\varepsilon}_{p} \geq 0 ; f\left(\boldsymbol{\sigma}_{n+1}, Y\left(\bar{\varepsilon}_{p_{n+1}}, \dot{\bar{\varepsilon}}_{p_{n+1}}, T_{n+1}\right)\right) \leq 0 ; f\left(\boldsymbol{\sigma}_{n+1}, Y(\ldots)\right) \Delta \bar{\varepsilon}_{p}=0
\end{gathered}
$$

Since the VUMAT material subroutine uses an explicit time-integration scheme, the temperature $T_{n+1}$ was updated in an explicit way for simplicity.

A two step-algorithm, called radial-return algorithm [28], was used to solve the problem. It is based on assuming a pure elastic step and checking the yield condition. If plastic flow occurs, the trial stress state is projected back on the yield surface. The following lines define how the radial-return algorithm was implemented:

1. Trial elastic state $(*)$.

$$
\begin{gathered}
\varepsilon_{n+1}^{e *}=\varepsilon_{n}^{e}+\Delta \varepsilon \\
\bar{\varepsilon}_{p_{n+1}}^{*}=\bar{\varepsilon}_{p_{n}} ; \dot{\bar{\varepsilon}}_{p_{n+1}}^{*}=\dot{\bar{\varepsilon}}_{p_{n}}^{*} ; T_{n+1}^{*}=T_{n}
\end{gathered}
$$

2. Trial stress state. Shear modulus $\mu=E /(2(1+\nu))$, bulk modulus $K=$ $E /(3(1-2 \nu))$, deviatoric part of the strain tensor $\varepsilon_{D_{n+1}}^{e *}$, volumetric part $\boldsymbol{\varepsilon}_{v_{n+1}}^{e *}$, deviatoric part of the stress tensor $\boldsymbol{\sigma}_{n+1}^{*}$ and hydrostatic part $\boldsymbol{\sigma}_{n+1}^{H *}$.

$$
\boldsymbol{\sigma}_{n+1}^{*}=2 \mu \varepsilon_{D_{n+1}}^{e *}+K \varepsilon_{v_{n+1}}^{e *}=\boldsymbol{\sigma}_{n+1}^{\prime *}+\boldsymbol{\sigma}_{n+1}^{H *}
$$

3. Checking yield condition.

$$
f\left(\boldsymbol{\sigma}_{n+1}^{*}, Y\left(\bar{\varepsilon}_{p_{n+1}}^{*}, \dot{\bar{\varepsilon}}_{p_{n+1}}^{*}, T_{n+1}^{*}\right)\right) \leq 0
$$

- If TRUE, the elastic guess is correct and all $(\bullet)_{n+1}^{*}=(\bullet)_{n+1}$

- ELSE:

$$
\begin{gathered}
\boldsymbol{\sigma}_{n+1}^{H *}=\boldsymbol{\sigma}_{n+1}^{H} \\
\boldsymbol{\sigma}_{n+1}^{\prime}=\boldsymbol{\sigma}_{n+1}^{\prime *}-2 \mu \Delta \bar{\varepsilon}_{p} \frac{3 \boldsymbol{\sigma}_{n+1}^{* *}}{2 \bar{\sigma}_{n+1}^{*}}
\end{gathered}
$$

where $\Delta \bar{\varepsilon}_{p}$ is calculated by solving, with the Newton-Raphson method, the non-linear equation:

$$
\bar{\sigma}_{n+1}^{*}-3 \mu \Delta \bar{\varepsilon}_{p}-Y\left(\bar{\varepsilon}_{p_{n+1}}, \dot{\bar{\varepsilon}}_{p_{n+1}}, T_{n+1}\right)=0
$$




\subsection{Cobalt-base superalloys}

In 2006, J. Sato et al. [71] discovered the possibility of having a stable twophase $\gamma / \gamma^{\prime}$ microstructure in Co-Al-W alloys. Since then, Co-base superalloys have become an interesting alternative to those based on $\mathrm{Ni}$ because of their good creep behaviour and superior corrosion resistance [72, 73, 74].

The model presented in Section 3.1 was applied to describe the dynamic behaviour of two Co-base superalloys at different temperatures, ranging from $298 \mathrm{~K}$ to $1123 \mathrm{~K}$. The material was manufactured by R. Casas et al. [75] through using the powder metallurgy route (PM) with two different compositions: Co-12Al$10 \mathrm{~W}$ (at.\%) for the hereafter called ternary alloy and Co-12Al-10W-2Ti-2Ta (at.\%) for the quinary alloy. Then, both alloys were homogenized (at $1573 \mathrm{~K}$ ) and aged (at $1173 \mathrm{~K}$ ) to achieve the $\gamma / \gamma^{\prime}$ microstructure, as can be seen in Figure 3.2. The volume fraction of $\gamma^{\prime}$ phase was $72 \%$ and $64 \%$ for the ternary and quinary alloy respectively.

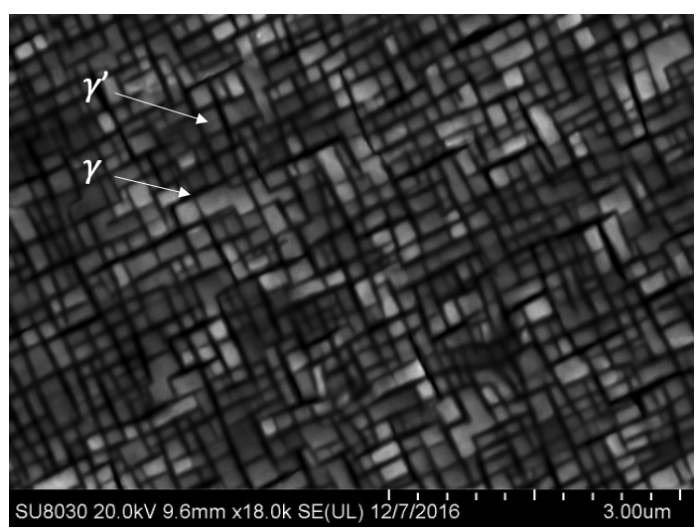

(a)

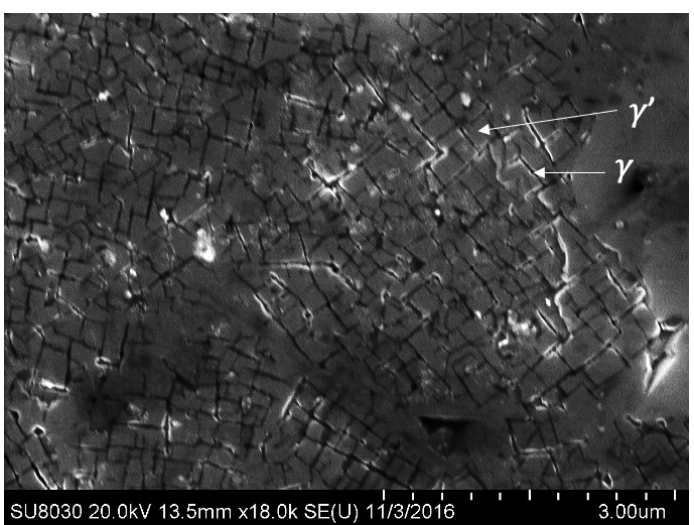

(b)

Figure 3.2: Secondary-electron micrographs showing the $\gamma / \gamma^{\prime}$ microstructure of the (a) Co-12Al-10W alloy and of the (b) Co-12Al-10W-2Ti-2Ta alloy (b). The cuboidal precipitates correspond to the $\gamma^{\prime}$ phase. Images by courtesy of Rafael Casas.

Dynamic uniaxial compression tests were carried out in the material (results already published in [76] by R. Casas) from room temperature to $1123 \mathrm{~K}$ by using the split-Hopkinson bar method. An engineering strain rate of $\dot{\mathrm{e}}=2500 \mathrm{~s}^{-1}$ 
was achieved in all experiments. The specimens were prismatic with dimensions of $2 \times 2 \times 4 \mathrm{~mm}^{3}$.

In the experimental set-up used, all bars had the same diameter $(10 \mathrm{~mm})$ while the length was equal to $480 \mathrm{~mm}$ for the striker bar and $1 \mathrm{~m}$ for the incident and transmission bars. The material of the bars was the René-41 nickel-base superalloy. The SHPB device was equipped with a SiC-resistance furnace (see Figure 3.3) to perform the high-temperature tests. The furnace heats up both the sample and some length of the end of the bars, so a water cooling system was employed to keep the strain gauges at room temperature. The temperature of the specimen was monitored through a thermocouple attached to it.

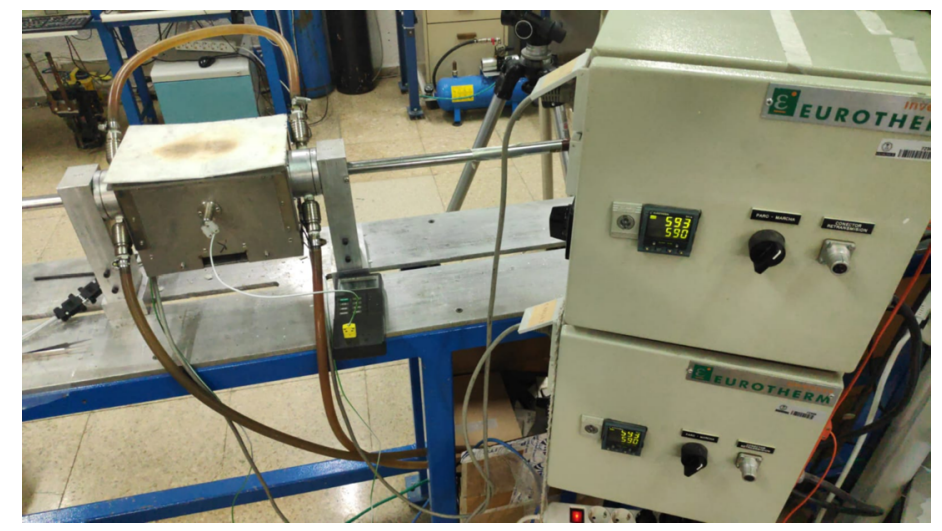

Figure 3.3: Split-Hopkinson pressure bar device with the furnace for testing at high temperatures.

The constitutive response of the samples was obtained through the equations presented in the Section 2.5. The effect of thermal gradients in the bar ends was neglected because the Young's modulus of the René-41 alloy is a relatively weak function of temperature $(218 \mathrm{GPa}$ at room temperature and $165 \mathrm{GPa}$ at $1144 \mathrm{~K}$ ) [77] and F. Gálvez demonstrated in [78] that there is no need of corrections. This approximation is a common procedure when testing highperformance alloys [79] .

The experimental stress-strain curves are plotted in Figure 3.4. Both alloys show a clear dependence of the flow stress with temperature, although the oscillations after the elastic part of the curves hinder the estimation of the temperature sensitivity of the yield strength. The origin of these oscillations is based on the combined effect of the Pochhammer-Chree wave dispersion, 
described in the Section 2.5, with the fact that a perfect bar-specimen contact is difficult. To overcome this problem, the yield-strength values were obtained after fitting, with the Scipy module[67] for Python programming language, the constitutive behaviour at room temperature and scaling down this curve for the rest of the temperatures in such a way that the error, between the constitutive equation and the experimental stress values, was minimised in the 0-0.05 strain range (dotted lines in Figure 3.4a). It may seem that this approach introduce some errors because, apparently, different hardening laws are needed for the different temperatures. However, it is a consequence of the thermal softening due to adiabatic heating and, as it will be demonstrated in the next section (numerical simulations), the strain hardening law $Y\left(\bar{\varepsilon}_{p}\right)$ can be assumed the same for all temperature ranges.

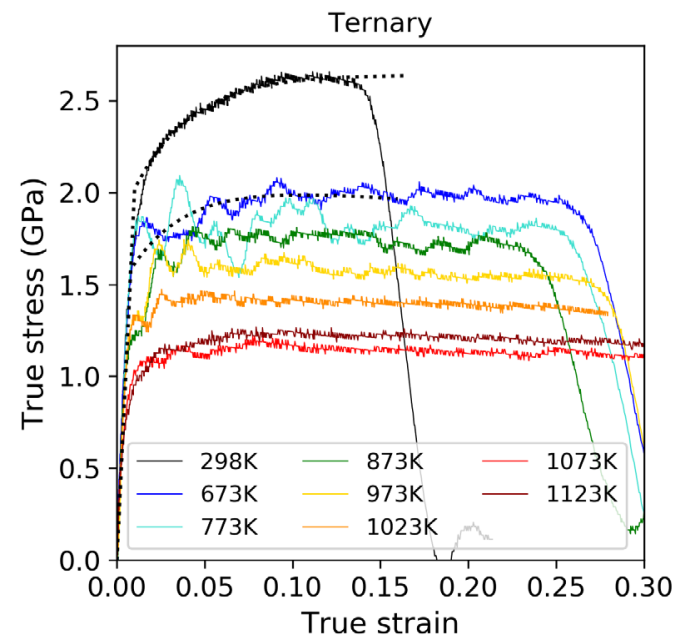

(a)

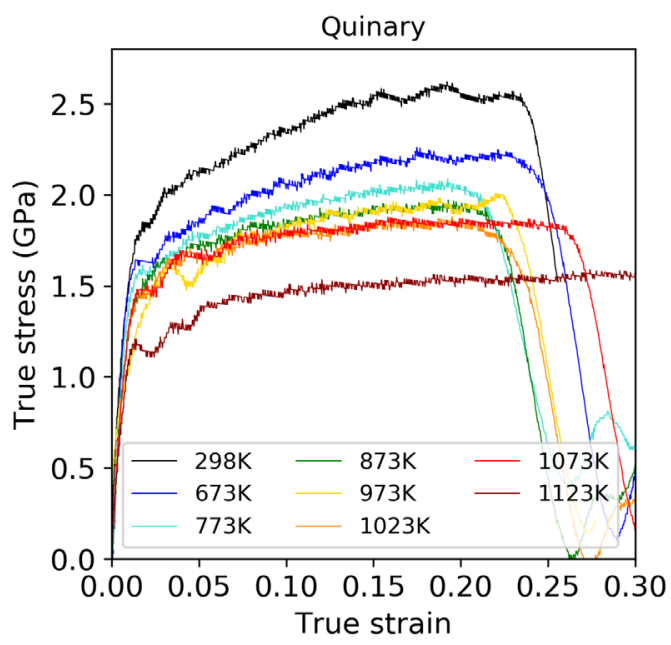

(b)

Figure 3.4: Adiabatic true stress-strain curves of (a) the ternary and (b) quinary alloys at $\dot{\mathrm{e}}=2500 \mathrm{~s}^{-1}$ for different temperatures.

The evolution of the yield strength with temperature, in conjunction with the adjustment of the proposed model, is plotted in Figure 3.5. It is important to note that the y-axis values are normalised with respect to the yield strength at room temperature; with $\sigma_{\text {ref }}=2.02 \mathrm{GPa}$ and $\sigma_{\text {ref }}=1.75 \mathrm{GPa}$ for the ternary and quinary alloys respectively.

Three different stages can be identified for both alloys. Firstly, from room 
temperature to $873 \mathrm{~K}$, the flow stress decay with temperature due to the thermal activation of dislocations. In the second stage, between $873 \mathrm{~K}$ and $973 \mathrm{~K}$ for the ternary alloy and between $873 \mathrm{~K}$ and 1073 for the quinary alloy, a bell-shaped stress peak is formed; being in agreement with observations in Co-Al-W alloys under quasi-static compression loading [6, 80, 68]. This anomalous behaviour has been demonstrated, in $[6,80]$, to be mainly caused by K-W locks (Section 2.4). According to [81], Ti and Ta alloying elements influence the APB energy, being the reason why the quinary alloy presents higher flow-strength values at high temperatures. Finally, the third stage displays again a decay of the yield strength when increasing the temperature due to the activation of cube slip on $\gamma^{\prime}$ and precipitate bypassing [80].

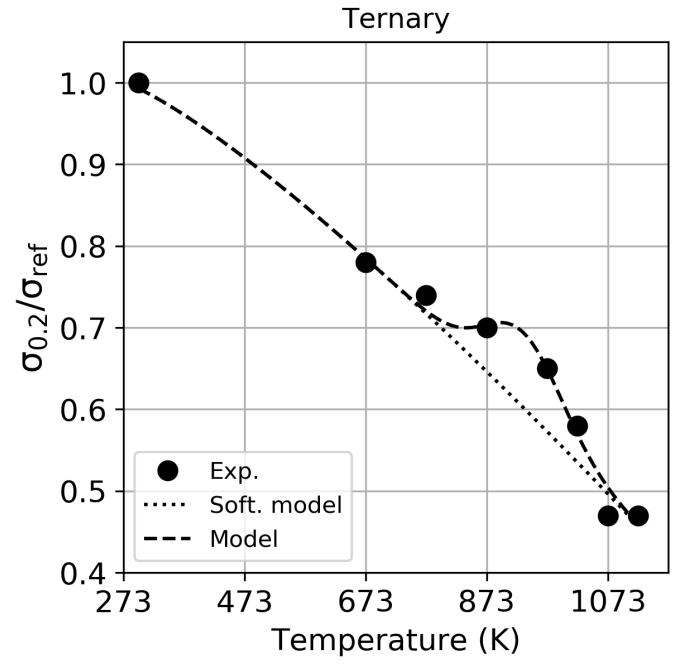

(a)

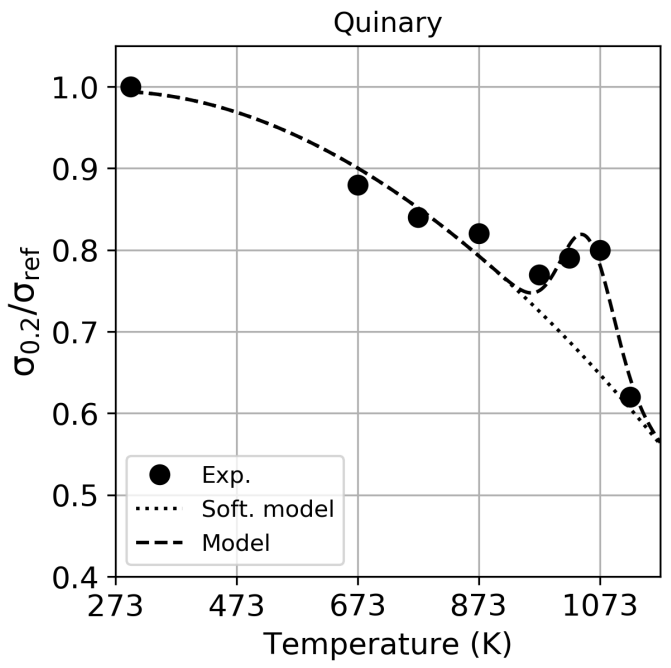

(b)

Figure 3.5: Temperature dependence of the dimensionless yield strength of (a) the ternary and (b) quinary Co-base alloys at $\dot{\mathrm{e}}=2500 \mathrm{~s}^{-1}$. Dashed line fits the experimental data (black dots) with the proposed model. Dotted line only considers the thermal-softening part of the model (Johnson-Cook type behaviour). $\sigma_{\text {ref }}$ is the yield strength at room temperature, $2.02 \mathrm{GPa}$ for the ternary alloy and 1.75 GPa for the quinary alloy. 


\subsubsection{Numerical simulations}

\section{Parameter identification}

Since the elastic and physical properties do not play an important role when modelling the flow stress, they were set as constant values for both alloys and temperature independent. The Young's modulus $E=220 \mathrm{GPa}$ and the density $\rho=9.47 \mathrm{~g} / \mathrm{cm}^{3}$ were obtained from [75]. The Poisson's ratio $\nu=0.3$ was chosen because it is a good value for metals and the specific heat capacity $C_{p}=420 \mathrm{~J} /\left(\mathrm{kg}^{\circ} \mathrm{C}\right)$ of cobalt was used. No information about the TaylorQuinney coefficient for Co-base alloys was found in the literature, so the generally assumed value of 0.9 was employed.

A strain-rate-sensitivity parameter $k=0.065$ was obtained, for both alloys, through comparing the yield strength of the studied alloys with the data given by other authors, T.M. Pollock et al. [80] and K. Shinagawa et al. [68], for the Co-Al-W alloy under compression quasi-static tests $\left(\dot{\varepsilon}_{0}=10^{-4}\right)$ (see Figure 3.6). Despite T.M. Pollock and K. Shinagawa tested materials with similar composition and microstructure (60-80\% of $\left.\gamma^{\prime}\right)$, the results were quite different. This deviation was taken into account to determine the strain-rate sensitivity parameter, assuming that the data presented by Pollock were the reference values for the rest of the yield-strength values (see Figure 3.6b).

The rest of the model parameters are gathered in Table 3.2 and Table 3.3. Hardening-law constants $\left(A, Q_{1}, C_{1}\right)$ were fitted with the data from the experiments at room temperature, while the constants that model the temperature dependence were adjusted with the data presented in Figure 3.5. As it can be seen, the proposed model (dashed lines) is able to describe the behaviour of both alloys. Quantitatively, the difference between the experimental yield strength and model predictions are in Table 3.4. It can be seen that error rates up to almost $20 \%$ can appear if the $\mathrm{K}-\mathrm{W}$ lock mechanism is not taken into account $\left(Y_{p 0}=0\right)($ dotted line in 3.5). 


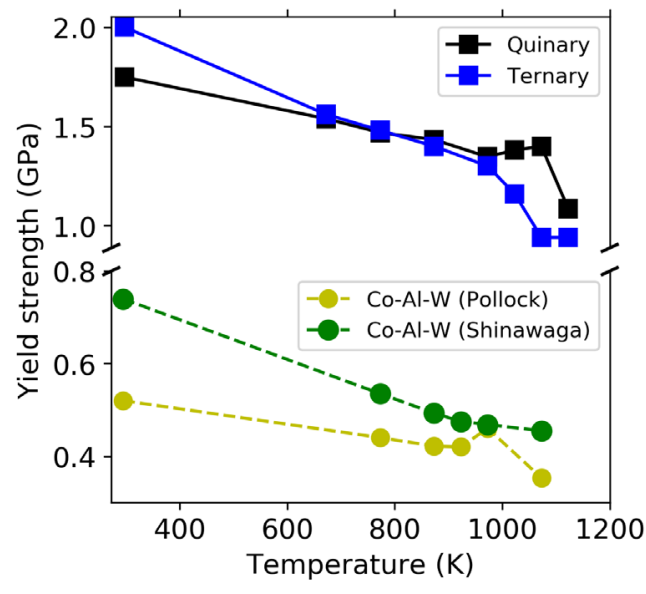

(a)

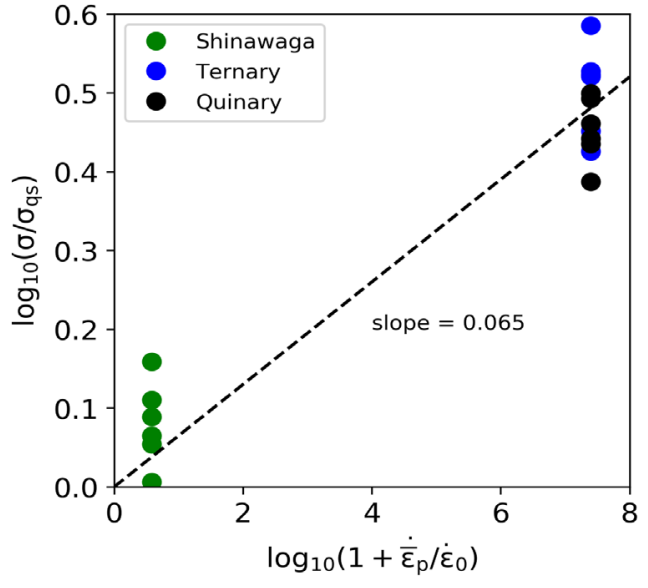

(b)

Figure 3.6: (a) Yield-strength comparison between the dynamic (present work) and quasi-static tests (other authors). (b) Estimation of the strain-rate sensitivity (slope of the straight line).

Table 3.2: Model parameters for the ternary alloy

\begin{tabular}{ccccccccc}
\hline$A(\mathrm{GPa})$ & $Q_{1}(\mathrm{GPa})$ & $C_{1}(-)$ & $T_{m}(\mathrm{~K})$ & $m_{t}(-)$ & $Y_{p 0}(\mathrm{GPa})$ & $T_{p}(\mathrm{~K})$ & $w_{p}(\mathrm{~K})$ & $n(-)$ \\
\hline 0.66 & 0.20 & 33 & 1673 & 1.22 & 0.22 & 930 & 120 & 1.9 \\
\hline
\end{tabular}

Table 3.3: Model parameters for the quinary alloy

\begin{tabular}{ccccccccc}
\hline$A(\mathrm{GPa})$ & $Q_{1}(\mathrm{GPa})$ & $C_{1}(-)$ & $T_{m}(\mathrm{~K})$ & $m_{t}(-)$ & $Y_{p 0}(\mathrm{GPa})$ & $T_{p}(\mathrm{~K})$ & $w_{p}(\mathrm{~K})$ & $n(-)$ \\
\hline 0.58 & 0.33 & 11 & 1673 & 1.89 & 0.28 & 1051 & 80 & 1.9 \\
\hline
\end{tabular}

Table 3.4: Error (\%) between the experimental yield strengths and the model predictions for each temperature

\begin{tabular}{ccccccccc}
\hline Temperature & $298 \mathrm{~K}$ & $673 \mathrm{~K}$ & $773 \mathrm{~K}$ & $873 \mathrm{~K}$ & $973 \mathrm{~K}$ & $1023 \mathrm{~K}$ & $1073 \mathrm{~K}$ & $1123 \mathrm{~K}$ \\
\hline Ternary model & -0.71 & 0.64 & -2.64 & 0.53 & -0.11 & 1.82 & 7.38 & -2.38 \\
Ternary $Y_{p 0}=0$ & -0.71 & 0.64 & -3.07 & -7.66 & -11.88 & -7.75 & -5.68 & -2.58 \\
Quinary model & -0.63 & 2.32 & 1.36 & -3.30 & -2.58 & 2.09 & 2.21 & 3.77 \\
Quinary $Y_{p 0}=0$ & -0.63 & 2.32 & 1.36 & -3.30 & -5.83 & -12.95 & -18.99 & -2.24 \\
\hline
\end{tabular}




\section{Simulations}

The SHPB tests were simulated with the Abaqus finite element code [66] to check the predictive capabilities of the model. Instead of simulating the whole experimental set-up, just the prismatic samples were taking into account. The sample geometry was discretised by using C3D8R rectangular hexahedron elements with dimensions of $0.1 \times 0.1 \times 0.15 \mathrm{~mm}$. The difference between the experimentally measured velocities of the bar ends were used to define the equations 3.21, 3.22 and prescribe the boundary conditions, getting good force agreement between experiments and simulations (see Figure 3.7). The simulations were run with the explicit solver by using the default time step calculated by Abaqus.

$$
\begin{gathered}
v_{z=L}=\left\{\begin{array}{l}
-1000 \cdot t \text { for } t \leq 0.01 \mathrm{~ms} \\
-10 \text { for } t>0.01 \mathrm{~ms}
\end{array} \mathrm{~mm} / \mathrm{ms}\right. \\
v_{x=y=z=0}=0 \mathrm{~mm} / \mathrm{ms}
\end{gathered}
$$

The numerical true stress-strain curves (see Figure 3.8 and Figure 3.9) were obtained from the reaction forces and the displacement developed on the nodes where the boundary condition $v_{z=L}$ was applied. The results show that the response of both specimens can be accurately predicted during sample deformation with the proposed model.

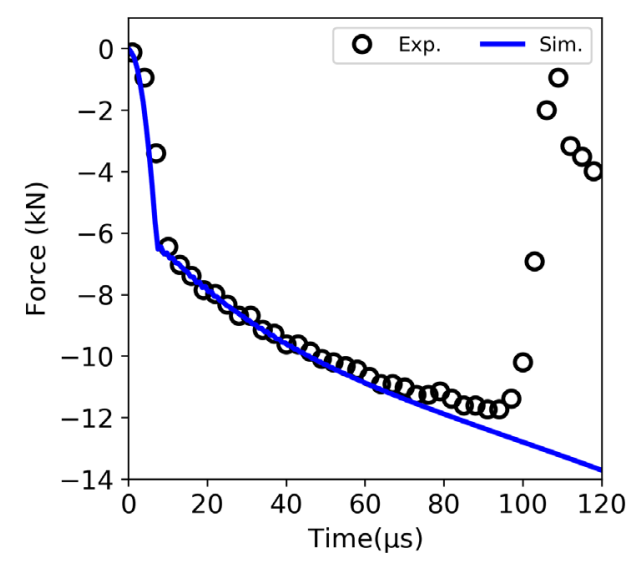

Figure 3.7: Comparison between the force applied to the specimens during the experimental campaign and the one obtained from simulation. Data of the quinary alloy at $673 \mathrm{~K}$ 


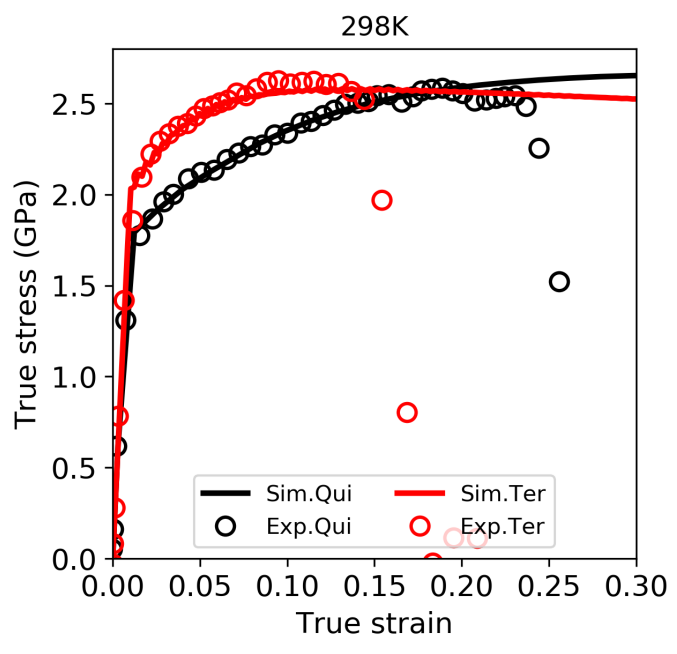

(a)

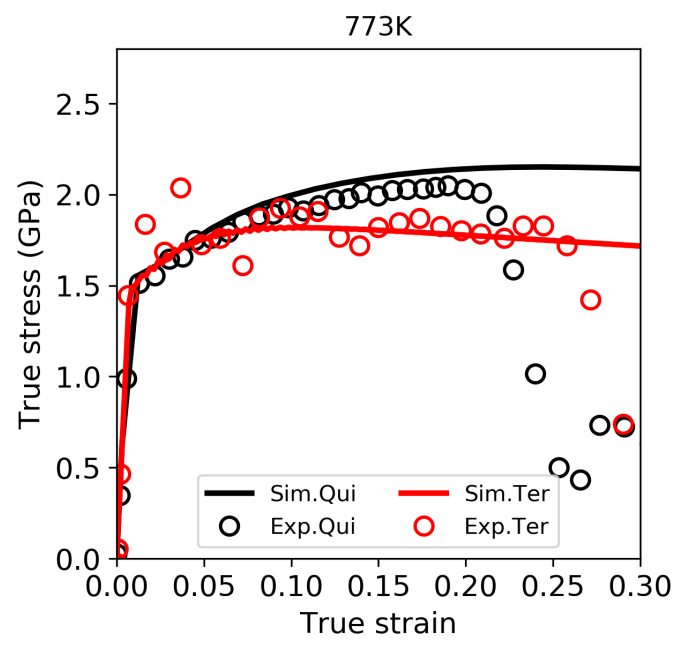

(c)

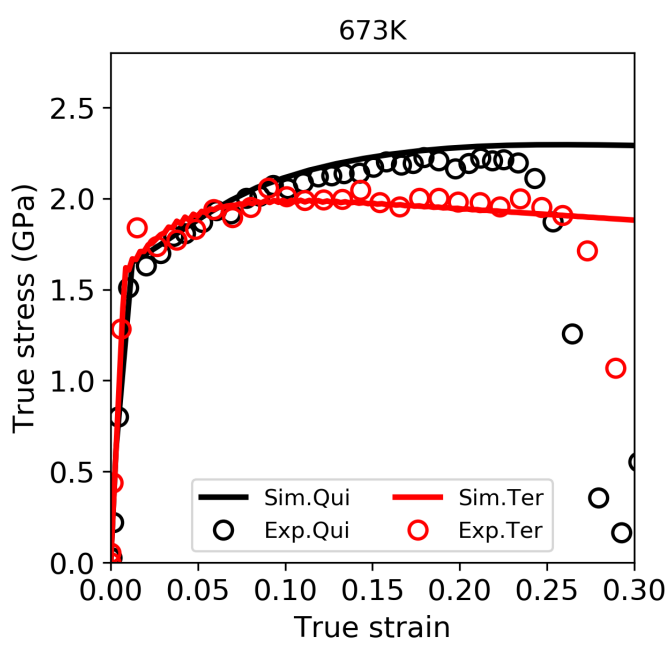

(b)

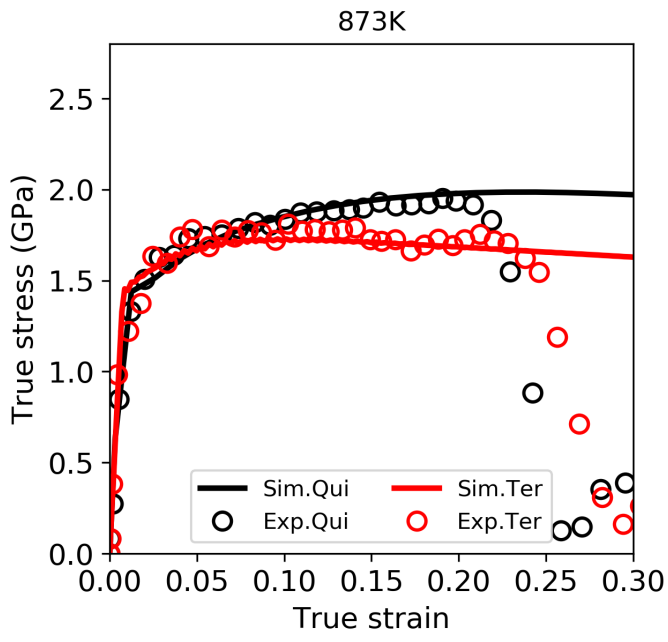

(d)

Figure 3.8: Experimental (circle markers) and simulated (solid lines) true stress-strain curves, from $298 \mathrm{~K}$ to $873 \mathrm{~K}$, for the ternary (red) and quinary (black) Co-base superalloys under high-strain-rate loading $\left(2500 \mathrm{~s}^{-1}\right)$. Each sub-figure corresponds to a different temperature. 


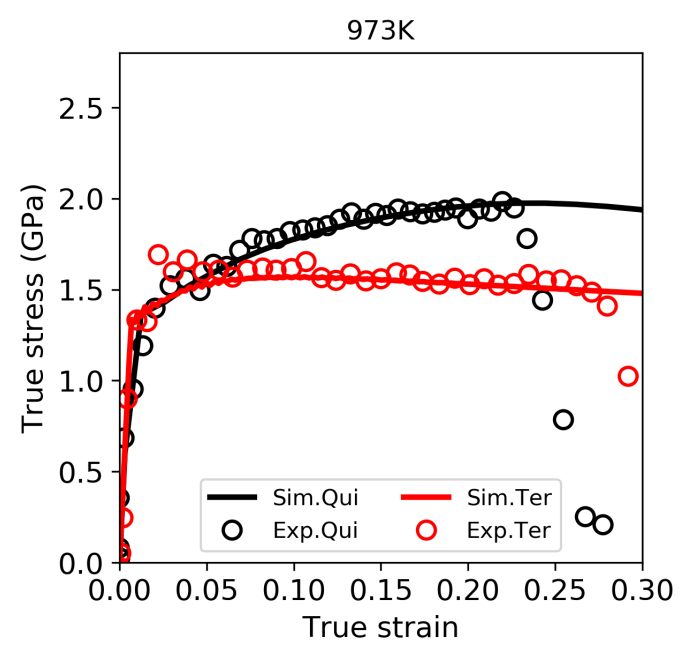

(a)

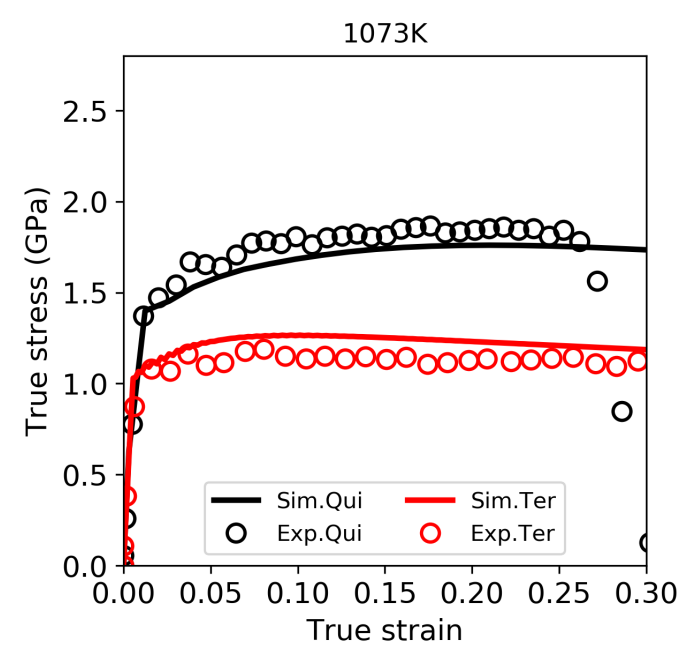

(c)

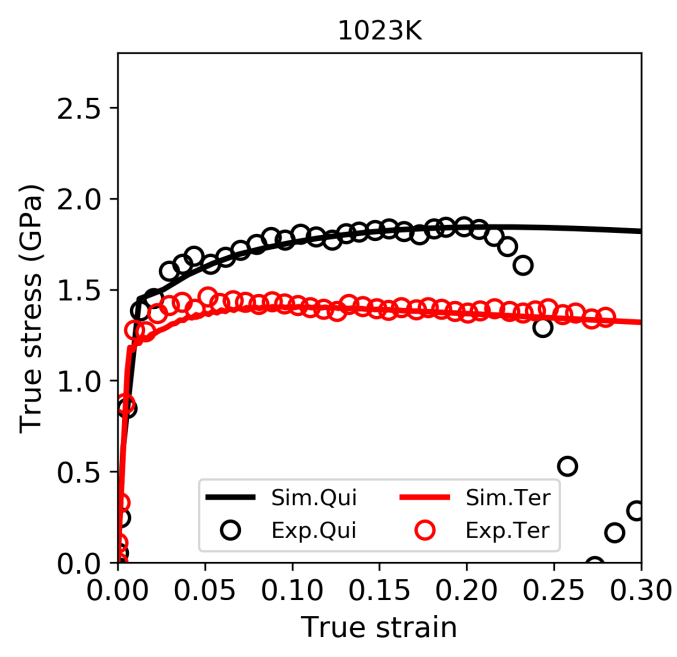

(b)

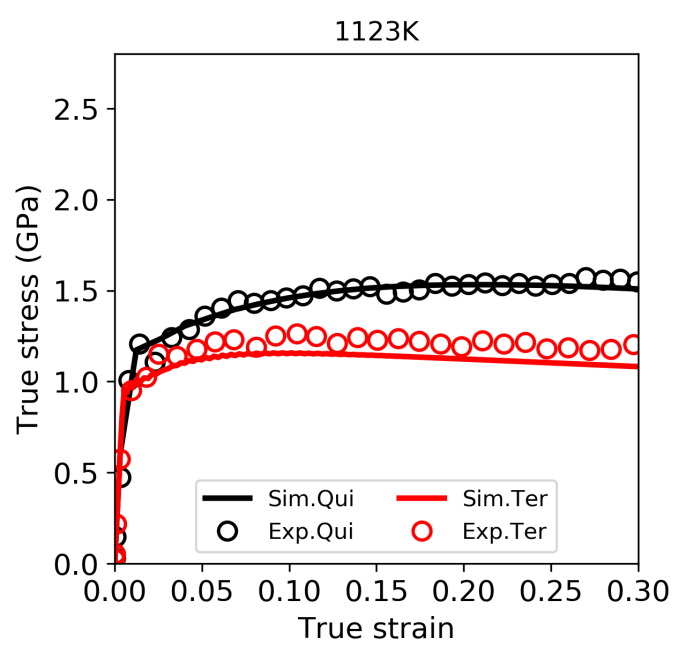

(d)

Figure 3.9: Experimental (circle markers) and simulated (solid lines) true stress-strain curves, from $973 \mathrm{~K}$ to $1123 \mathrm{~K}$, for the ternary (red) and quinary (black) Co-base superalloys under high-strain-rate loading $\left(2500 \mathrm{~s}^{-1}\right)$. Each sub-figure corresponds to a different temperature. 


\section{Importance of peak flow stress during ballistic applications}

To analyse the consequences of not considering the peak flow stress, whether by not taking into account enough temperatures during experimental testing or by fitting data with an unsuitable model, such as the traditional Johnson-Cook model, some ballistic impact simulations were done.

The virtual tests were defined as the impact, at $1073 \mathrm{~K}$, of a blunted cylindrical projectile against a disc-shaped plate made of the quinary alloy (see Figure 3.10). That temperature was chosen because it is the most critical situation if the peak flow stress is not considered (see Table 3.4) and is also achieved in the turbine zone of aircraft engines [82].

To reduce the computational cost, axisymmetric elements, with a size of $0.1 \times 0.1 \mathrm{~mm}$ were used. The projectile was modelled with a length and a radius of $31 \mathrm{~mm}$ and $3 \mathrm{~mm}$ respectively, and considering a linear-elastic material with $E=220 \mathrm{GPa}$ and $\nu=0.3$. The dimensions of the plate, radius of $10 \mathrm{~mm}$ and thickness of $2.2 \mathrm{~mm}$, were defined according to [10]. It is important to note that all these properties could be re-defined since the simulations only seek to compare the performance of the material plate between two situations: considering and not considering the peak flow stress.

The goal of an impact tests is to determine the ballistic limit of the structure; being defined as the velocity of the projectile with $50 \%$ of perforation probability. Hence, a damage condition was also defined. Ductile damage models strongly depend on the stress triaxiality [83] but we do not have such information for our alloy. For the sake of simplicity, we used an equivalent-plasticstrain-to-failure damage model that erodes the element when the accumulated plastic strain $\bar{\varepsilon}_{p}$ equals the critical plastic strain EPS fail. According to the experimental test (Figure 3.4), a value of EPS fail $=0.25$ was defined .

Figure 3.11 depicts the results of the ballistic simulations. It can be seen that not taking into account the peak flow stress on the performance of the material decreases the ballistic limit by a $6 \%$, from $148 \mathrm{~m} / \mathrm{s}$ (blue line) to $139 \mathrm{~m} / \mathrm{s}$ (red line). 


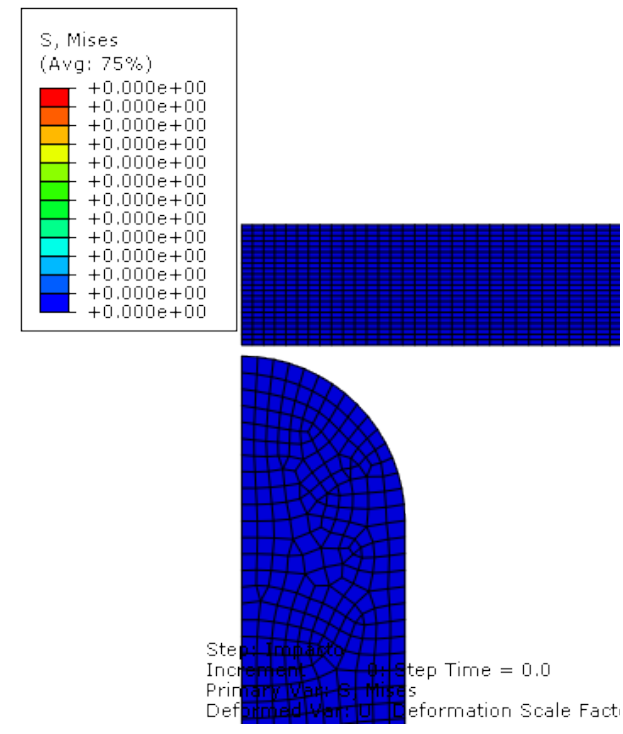

(a)

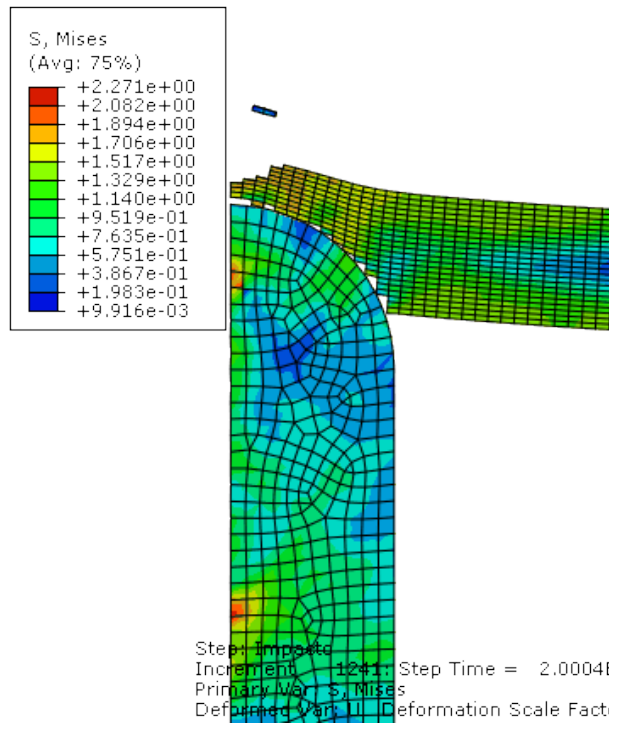

(b)

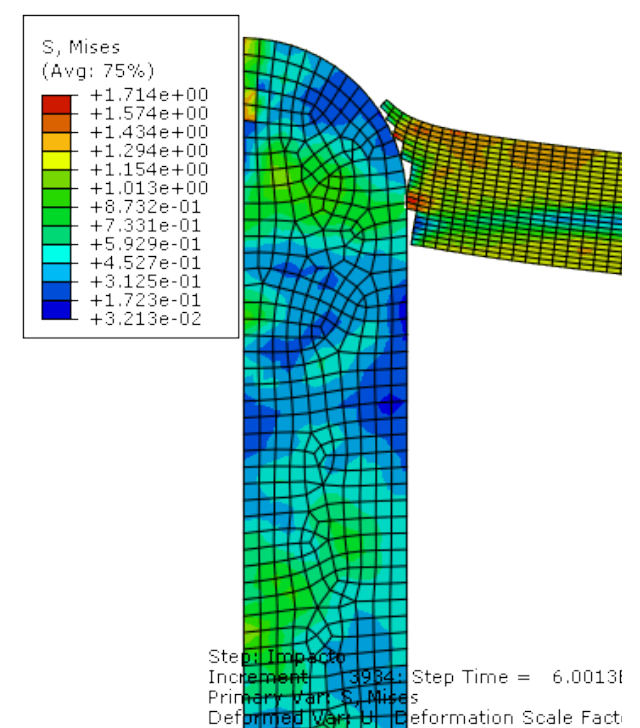

(c)

Figure 3.10: Frames of the virtual impact test: (a) before, (b) during impact and (c) after plate perforation 


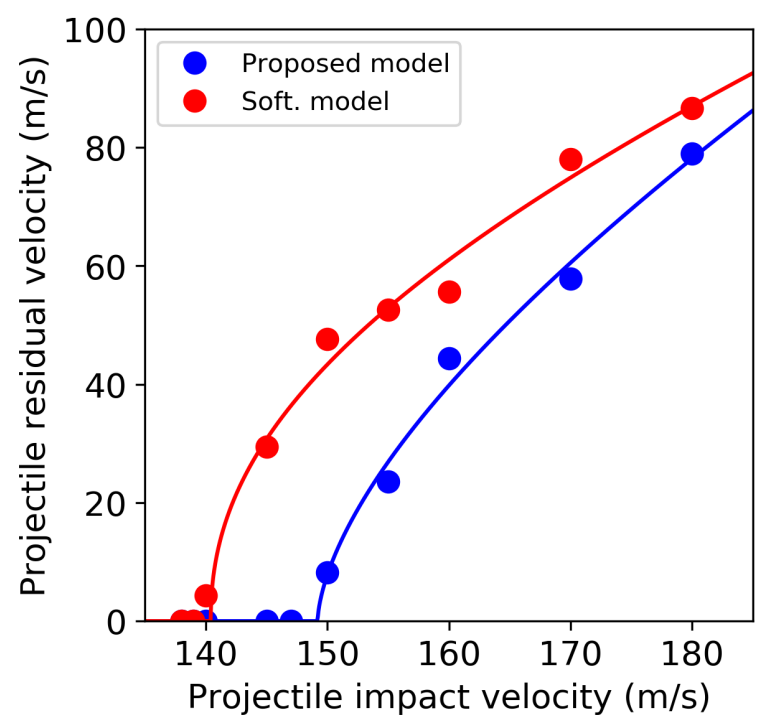

Figure 3.11: Results of the virtual impact tests. Red dots represent the results without modelling the peak-flow stress while blue dots represent the results considering the peak-flow stress. 


\section{$3.3 \quad$ VascoMax C-250}

VascoMax C-250 is an alloy that belongs to the family of maraging steels, characterised by a precipitation-hardening martensite microstructure. The term "maraging" is derived from the words "martensite" and "age hardening". This martensite has a body-centred cubic lattice and it is based on the Fe-Ni phase diagram (Figure 3.12), being more ductile and tougher than the one formed under the Fe-C phase diagram [84]. Its excellent weldability, high fracture toughness and good workability has led to the use of maraging steel in aerospace applications such as rocket motor cases and helicopter drive shafts [85].

The chemical composition of the alloy, according to the material supplier [85], is listed in Table 3.5. As it can be seen, nickel is the main alloying element and its role is to form, with iron atoms, the martensite after annealing and air cooling the alloy. Molybdenum is responsible for the precipitation of the $\mathrm{Ni}_{3} \mathrm{Mo}$ intermetallic compound while cobalt decreases the solubility of molybdenum in the martensite microstructure with the aim of increasing the amount of precipitates [84].
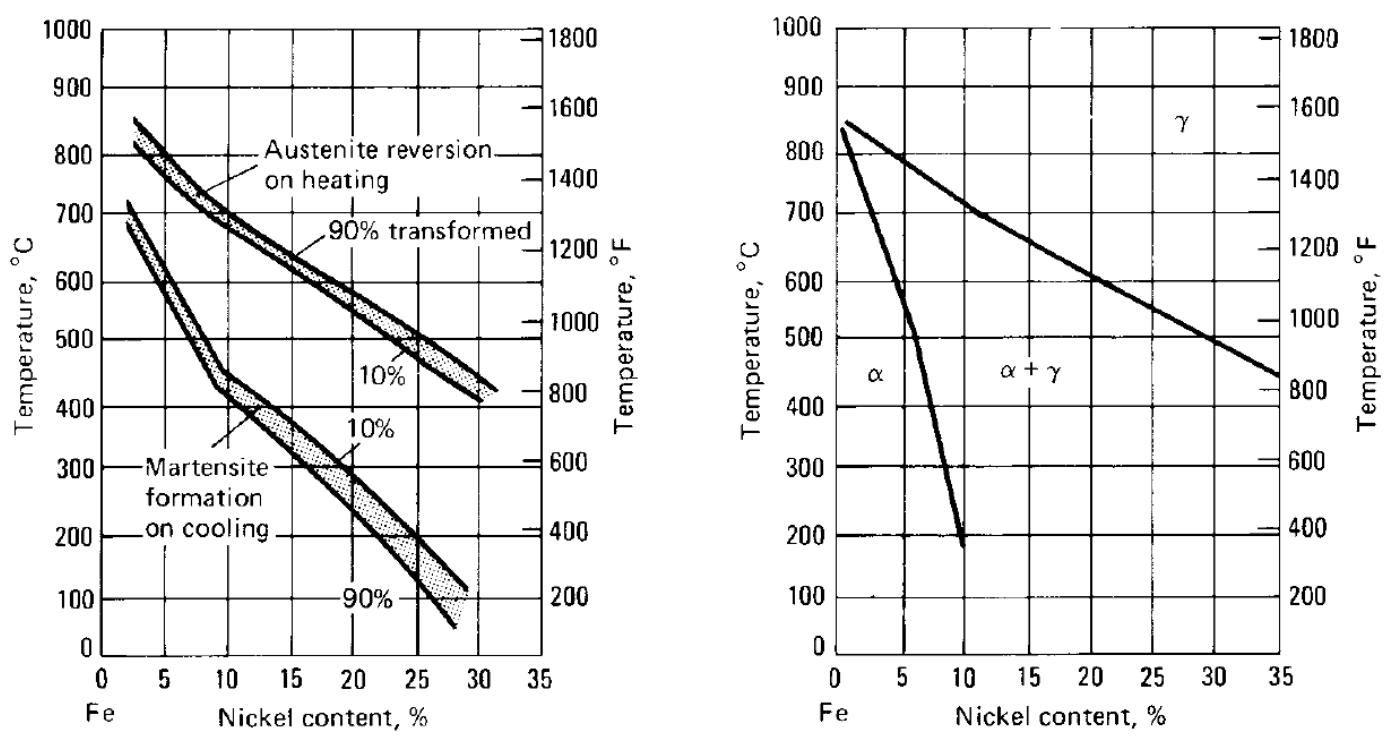

Figure 3.12: Metastable and equilibrium phases in the Fe-Ni system. Image obtained from [84]. 
Chapter 3. A model for the yield-strength anomaly

Table 3.5: Chemical composition (\%wt.) of the alloy VascoMax C-250.

Data obtained from the supplier.

\begin{tabular}{cccccccc}
\hline $\mathrm{Ni}$ & $\mathrm{Co}$ & $\mathrm{Mo}$ & $\mathrm{Ti}$ & $\mathrm{Al}$ & $\mathrm{Si}$ & $\mathrm{Mn}$ & $\mathrm{C}$ \\
\hline 18.5 & 7.50 & 4.80 & 0.40 & 0.10 & 0.10 & 0.10 & 0.03 \\
\hline
\end{tabular}

Split-Hopkinson pressure bar tests were performed on the material, at an engineering strain rate of $2500 \mathrm{~s}^{-1}$, from ambient temperature $(298 \mathrm{~K})$ to $967 \mathrm{~K}$, applying a heating rate of $8 \mathrm{~K} / \mathrm{min}$ and a temperature-stabilization time of 5 minutes. Prismatic samples, with dimensions of $6 \times 4 \times 4 \mathrm{~mm}^{3}$, were machined from a $20 \mathrm{~mm}$-diameter bar in the annealed condition, i.e., martensite microstructure without precipitates.

The basis of SHPB tests and its basic configuration are explained in detail in Section 2.5. In the present case, all bars (striker, incident and transmission bar) had a diameter of $19.3 \mathrm{~mm}$ and were made of René-41 alloy. The length of the projectile was equal to $37 \mathrm{~cm}$ and the length of the incident and transmission bars was equal to $1 \mathrm{~m}$. The high temperature tests were performed by mounting a SiC-resistor furnace, the same as that used for the Co-base superalloys (Figure 3.3 in Section 3.2). The image shows the heat sinks on the bars to avoid the damage of the strain gauges, the thermocouple to control the specimen temperature and the Eurotherm temperature controller. The effect of temperature on the stress-waves propagation was disregarded during the signal analysis of high-temperature tests. The reason was already justified in Section 3.2 .

The adiabatic true stress-strain curves obtained from the experimental campaign are gathered in Figure 3.13. It is important to note that three experimental curves were plotted with markers for the sake of legibility (results at $635 \mathrm{~K}$ and $655 \mathrm{~K}$ in the sub-figure 3.13b and result at $683 \mathrm{~K}$ in the sub-figure 3.13c). Observing the curves, the temperature dependence of the flow stress of the material is clear and three different regions can be identified (Figure 3.14). From $298 \mathrm{~K}$ to $573 \mathrm{~K}$ (first stage), the material decreases its resistance against plastic deformation when increasing the temperature because of the thermal activation of dislocations. Then, its performance starts to increase continuously up to the temperature range of $800-895 \mathrm{~K}$ (second stage). In this case, the yield strength 


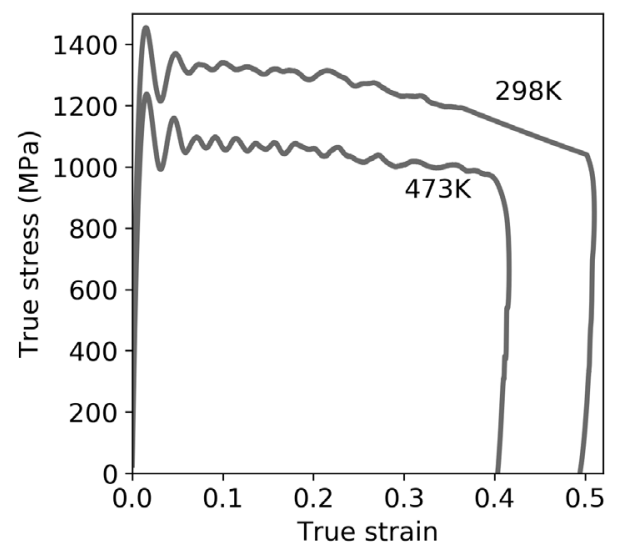

(a)

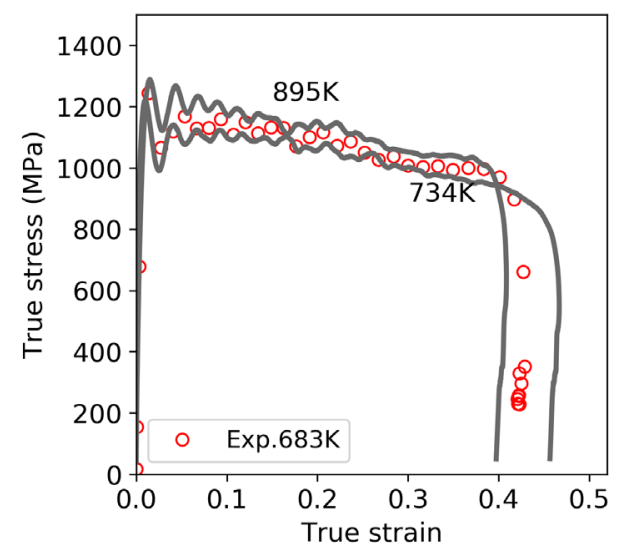

(c)

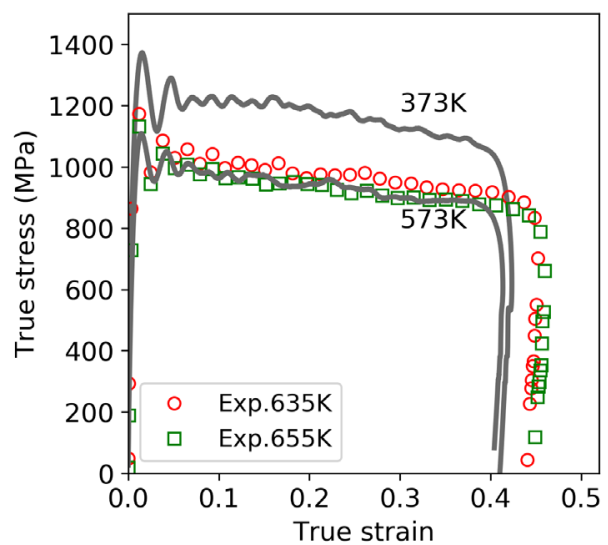

(b)

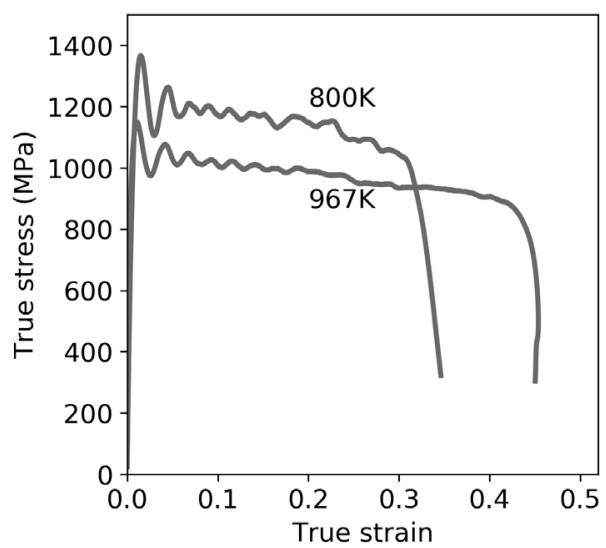

(d)

Figure 3.13: Adiabatic true stress-strain of the dynamic compression tests for the different temperatures: (a) $298 \mathrm{~K}$ and $473 \mathrm{~K}$, (b) $373 \mathrm{~K}, 573 \mathrm{~K}, 635$ $\mathrm{K}$ and $655 \mathrm{~K}$, (c) $683 \mathrm{~K}, 734 \mathrm{~K}$ and $895 \mathrm{~K}$ and (d) $800 \mathrm{~K}$ and $967 \mathrm{~K}$.

anomaly is not related to the Kear-Wilsdorf mechanism but to a process of precipitation hardening. Figure 3.15 shows the influence of temperature and ageing time on the hardness evolution of the alloy. It can be seen that precipitation hardening starts above $588 \mathrm{~K}$ and that the increase of hardness occurs for even short ageing times (less than 6 minutes). The samples tested at $635 \mathrm{~K}$ and $655 \mathrm{~K}$ stays above $588 \mathrm{~K}$ for $11-14$ minutes (heating + stabilization), being enough time to compensate the thermal softening with the ageing hardening. Beyond these temperatures, the amount of precipitates rises because the tem- 
perature and the ageing time increase and therefore, the yield strength evolution starts to be positive. Finally, at $895 \mathrm{~K}$ (third stage), the material enters in the austenite region (Figure 3.12) and decrease its performance.

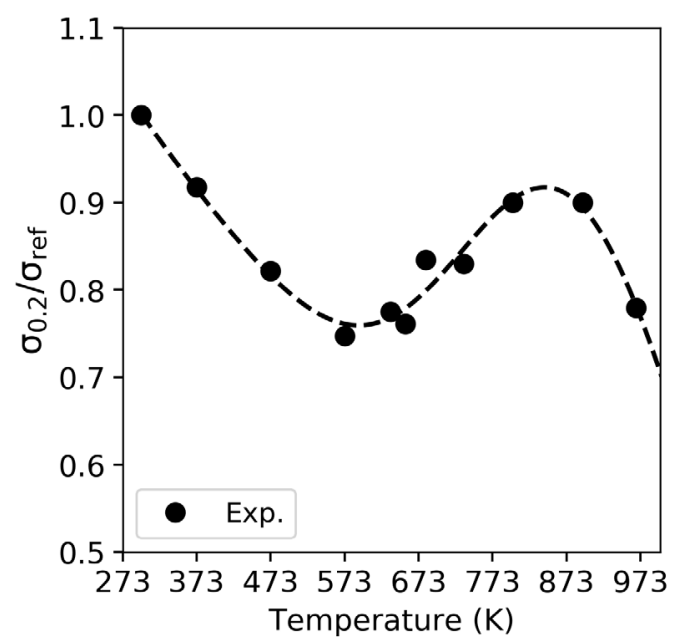

Figure 3.14: Temperature dependence of the dimensionless yield strength of VascoMax C-250 in the annealed condition at $\dot{\mathrm{e}}=2500 \mathrm{~s}^{-1} . \sigma_{\text {ref }}=1320 \mathrm{MPa}$ is the yield strength value at room temperature

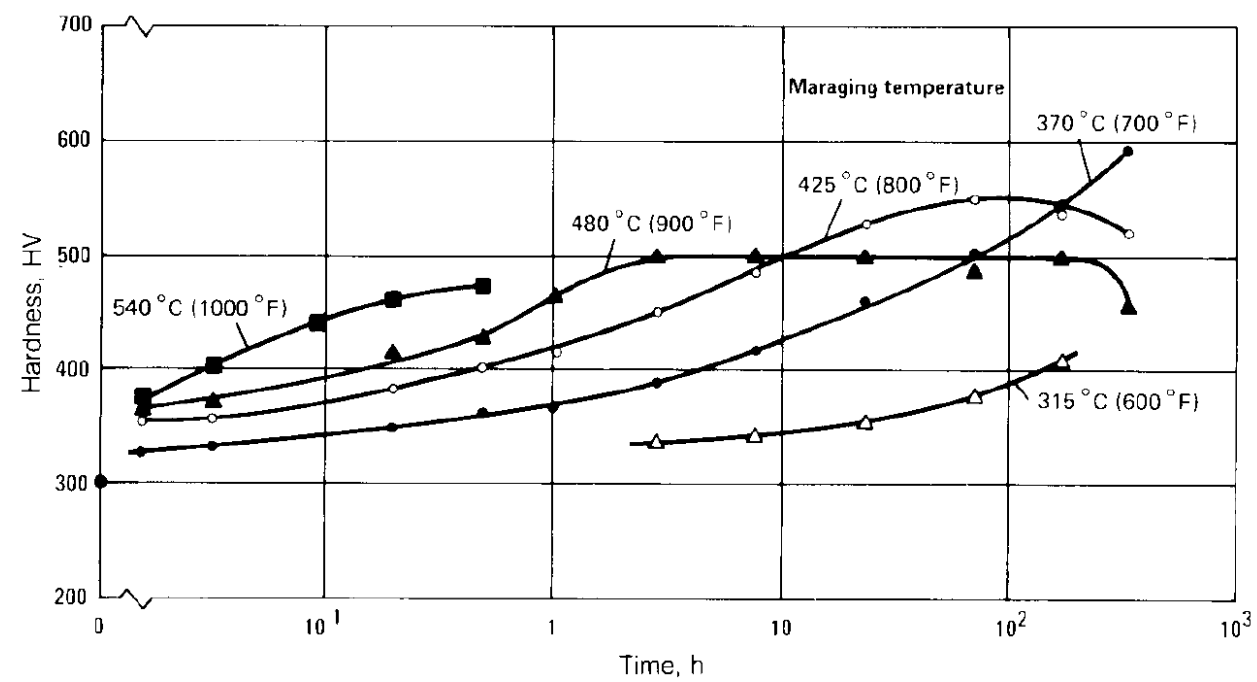

Figure 3.15: Hardness evolution of the maraging steel C-250 depending on the temperature and ageing time (precipitation heat treatment). Graph obtained from [84] 


\subsubsection{Numerical simulations}

\section{Parameter identification}

Figure 3.16a clearly shows the evolution of the yield strength of the steel with temperature. Those data were used to fit, by using a non-linear least square procedure, implemented in the Scipy library for Python programming language [67], the constants of the proposed model that define the temperature dependence $\left(T_{m}, m_{t}, Y_{p 0}, T_{p}, w_{p}\right.$ and $n$ in Table 3.6). It should be noted that since precipitation kinetics is in the order of minutes, adiabatic heating does not play any role in the hardening of the material. Therefore, in this case, the temperature variable of the function $\Delta Y_{p}(T)$ of the YSA model (see equation 3.8) was set as non-sensitive to adiabatic heating during numerical simulations.

The hardening-law parameters $\left(A, Q_{1}\right.$ and $C_{1}$ in Table 3.6) were identified from the response of the alloy at ambient temperature. To that end, the isothermal true stress-strain curve was calculated and its elastic part was removed $\left(\varepsilon_{e}=0.009\right)$, as can be seen in Figure 3.16b. The isothermal curve is nothing but the experimental curve after correcting the softening effect due to adiabatic heating. The next steps were followed:

- Obtain the softening behaviour of the alloy $\frac{\sigma_{0.2}}{\sigma_{r e f}}(T)$. $\sigma_{0.2}$ stands for the yield strength and $\sigma_{r e f}$ for the reference yield strength (at room temperature).

- Calculate the temperature evolution $T\left(\bar{\varepsilon}_{p}\right)$ as a function the plastic strain (equation 2.39).

- Correct the true-stress curve $\sigma\left(\bar{\varepsilon}_{p}\right)$ with the relationship $\frac{\sigma_{0.2}}{\sigma_{r e f}}\left(T\left(\bar{\varepsilon}_{p}\right)\right)$

The rest of material constants were obtained from bibliographic resources. The Young's modulus $E=186 \mathrm{GPa}$, Poisson's ratio $\nu=0.3$ and the density $\rho=8000 \mathrm{~kg} / \mathrm{m}^{3}$ were obtained from the material supplier [85]. The specific heat $C_{p}=293 \mathrm{~J} / \mathrm{kg} / \mathrm{K}$ was defined according to [86] while the Taylor-Quinney coefficient was set to 0.7 [87]. 


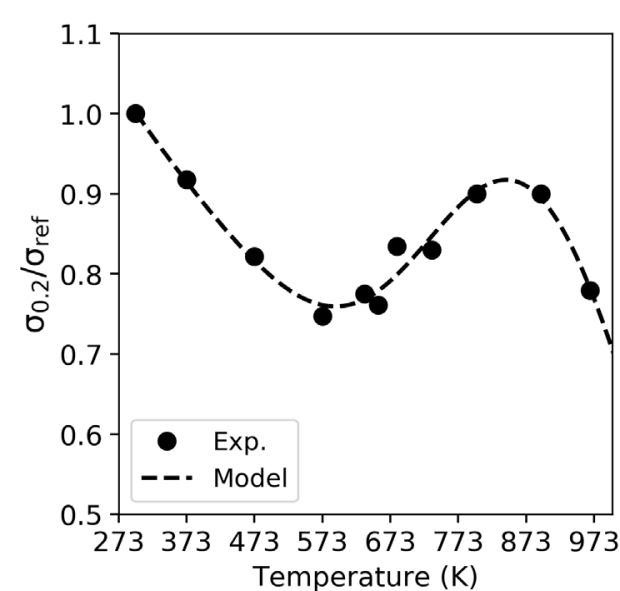

(a)

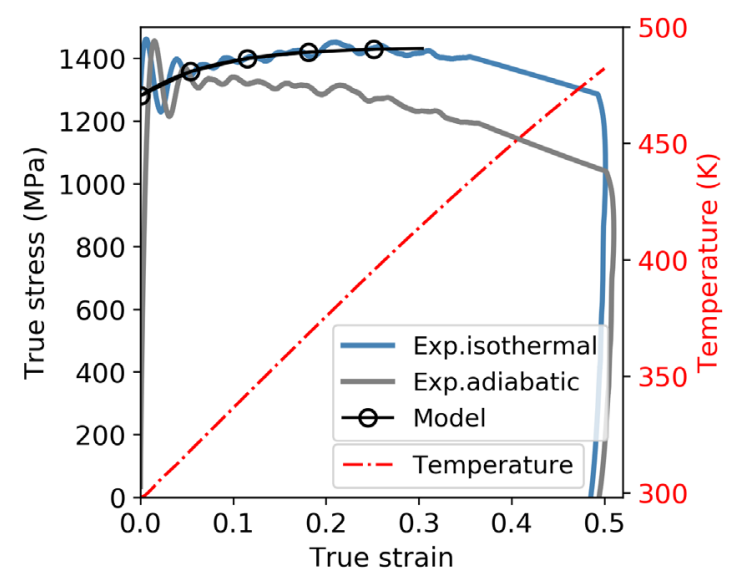

(b)

Figure 3.16: (a) Temperature dependence of the dimensionless yield strength of VascoMax C-250 in the annealed condition at $\dot{\mathrm{e}}=2500 \mathrm{~s}^{-1}$. $\sigma_{\text {ref }}=1320 \mathrm{MPa}$ is the yield strength value at room temperature. (b) Effect of the thermal softening due to adiabatic heating in the hardening law of the alloy at room temperature.

Table 3.6: Model parameters for the steel VascoMax C-250 in the annealed condition.

\begin{tabular}{ccccccccc}
\hline$A(\mathrm{GPa})$ & $Q_{1}(\mathrm{GPa})$ & $C_{1}(-)$ & $T_{m}(\mathrm{~K})$ & $m_{t}(-)$ & $Y_{p 0}(\mathrm{GPa})$ & $T_{p}(\mathrm{~K})$ & $w_{p}(\mathrm{~K})$ & $n(-)$ \\
\hline 1.03 & 0.12 & 12.9 & 1273 & 0.93 & 0.71 & 882 & 259 & 1.43 \\
\hline
\end{tabular}

Finally, the strain-rate-sensitivity exponent $k=0.0147$ was estimated through the ratio:

$$
k=\frac{\ln \left(\frac{1320 M P a}{1027 M P a}\right)}{\left.\ln \left(1+\frac{2500 s^{-1}}{10^{-4} s^{-1}}\right)\right)}
$$

where the value of the yield strength in the annealed condition (1027 MPa) at room temperature and quasi-static loading was obtained from [85]. 


\section{Simulations}

With the aim of examining the capabilities of the proposed model, numerical simulations of the experimental campaign were performed by using the Abaqus FEA code [66].

The specimens were discretised through using eight-node brick elements with reduced integration (C3D8R). Twenty elements were used along each direction, giving an element size of $0.2 \times 0.2 \times 0.2 \mathrm{~mm}^{3}$. To save computational time, the boundary conditions were applied directly on the sample surfaces without the need for the incident and the transmission bars. The displacement of the nodes corresponding to the end $z=0$ was constrained while the prescribed velocity profile described in the equation 3.24 was imposed to the opposite-end nodes. The values of the equation were defined to replicate the experimental boundary conditions. Moreover, to avoid undesired translations and rotations of the sample, the displacement of the nodes on the planes $x=0$ and $y=0$ were set equal to zero in the $\mathrm{x}$ - and $\mathrm{y}$-direction respectively. The simulations were run with the explicit solver by using the default time step calculated by Abaqus.

$$
v_{z=L}=\left\{\begin{array}{l}
-776.7 \cdot t \text { for } t \leq 0.0206 \mathrm{~ms} \\
-16 \text { for } t>0.0206 \mathrm{~ms}
\end{array} \mathrm{~mm} / \mathrm{ms}\right.
$$

Finally, the mechanical response of the simulated material were computed from the reaction forces and the displacement registered by the nodes on the plane $z=L$, where $L$ is the length of the prismatic specimen. The true stressstrain curves are plotted in Figure 3.17 (dashed lines) to be compared with the experimental ones. It can be seen that the presented material model is able to replicate the experimental curves with high accuracy for all temperatures. 


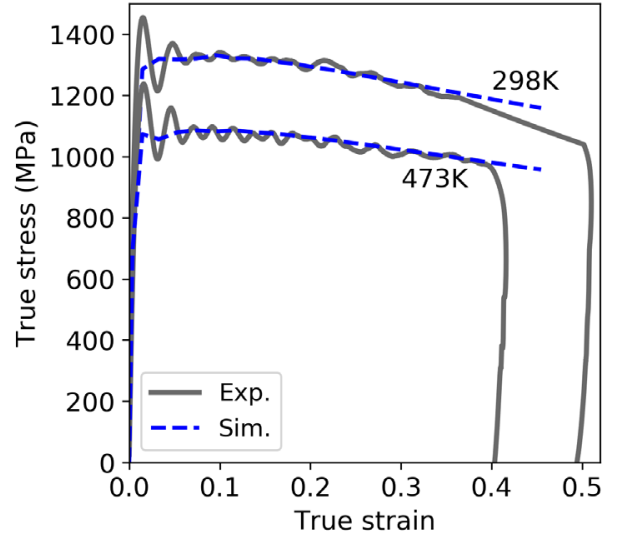

(a)

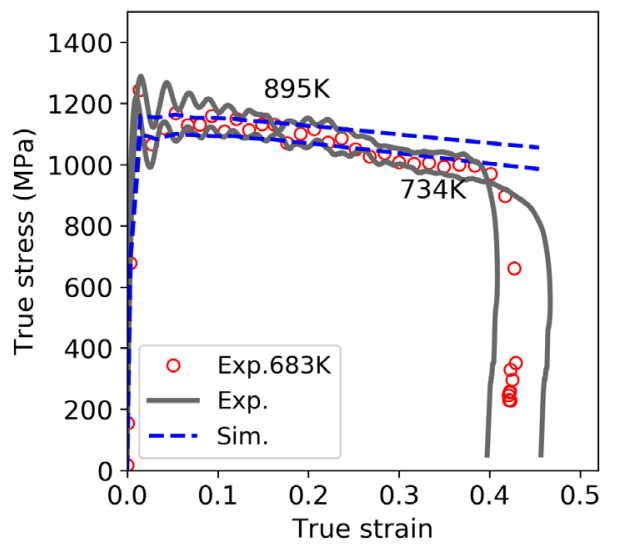

(c)

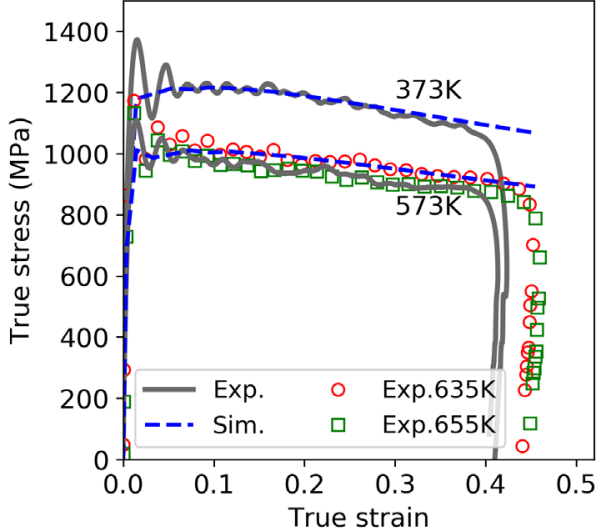

(b)

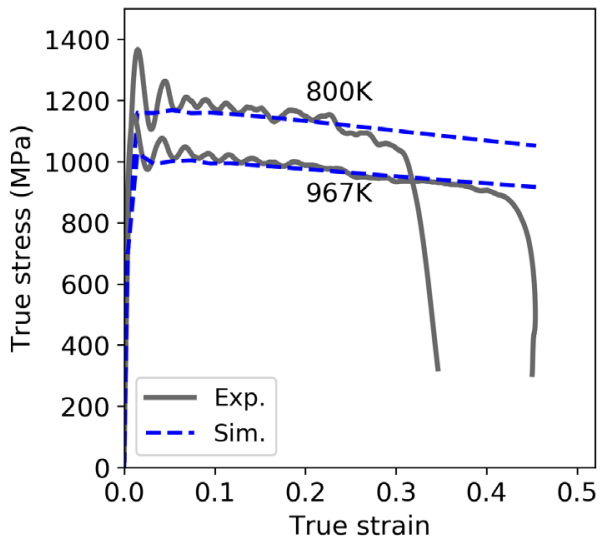

(d)

Figure 3.17: Comparison between the adiabatic true stress-strain of the experimental and numerical dynamic compression tests for different temperatures: (a) $298 \mathrm{~K}$ and $473 \mathrm{~K}$, (b) $373 \mathrm{~K}, 573 \mathrm{~K}, 635 \mathrm{~K}$ and $655 \mathrm{~K}$, (c) $683 \mathrm{~K}$, $734 \mathrm{~K}$ and $895 \mathrm{~K}$ and (d) $800 \mathrm{~K}$ and $967 \mathrm{~K}$. 


\subsection{MAR-M247}

MAR-M247 is a casting nickel-base superalloy that was developed in the early $1970_{\text {s }}$ for turbine blades and vane parts by Martin Metals Corporation [88]. Its microstructure consists of a solid-solution-strengthened matrix ( $\gamma$ phase) with a volume fraction around $60 \%$ of $\mathrm{Ni}_{3}(\mathrm{Ti}, \mathrm{Al})$ precipitates $\left(\gamma^{\prime}\right.$ phase). As mentioned in Section 2.4, the $\gamma$ phase has and FCC lattice while the precipitates have an $\mathrm{L}_{2}$ ordered structure. The nominal chemical composition, given by the material supplier GKN Aerospace, can be seen in Table 3.7.

Table 3.7: Chemical composition of MAR-M247 (\%wt.).

\begin{tabular}{cccccccccc}
\hline $\mathrm{Cr}$ & $\mathrm{Co}$ & $\mathrm{Al}$ & $\mathrm{Ti}$ & $\mathrm{W}$ & $\mathrm{Ta}$ & $\mathrm{Mo}$ & $\mathrm{C}$ & $\mathrm{Hf}$ & $\mathrm{Ni}$ \\
\hline 8.00 & 10.0 & 5.50 & 1.00 & 10.0 & 3.00 & 0.60 & 0.15 & 1.50 & Bal. \\
\hline
\end{tabular}

The alloy was manufactured by directional solidification. The bulk material was cut into three different prismatic samples of size $12 \times 15 \times 12 \mathrm{~mm}^{3}$ to study the microstructure by optical microscopy. The samples were polished up to $1 \mu \mathrm{m}$ and etched with a solution of $1 \mathrm{~mL}$ of $\mathrm{H}_{2} \mathrm{O}_{2}+2 \mathrm{~mL}$ of $\mathrm{HCl}+3 \mathrm{~mL}$ of $\mathrm{H}_{2} \mathrm{O}$ for one minute to reveal the microstructure, proving the existence of columnar grains with sizes in the range of several millimetres (Figure 3.18). The images were obtained by using an Optech LFZ zoom stereomicroscope equipped with a ProgRes digital camera.

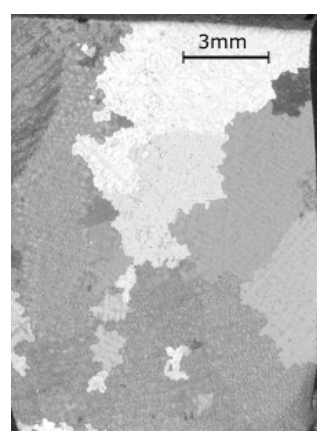

(a)

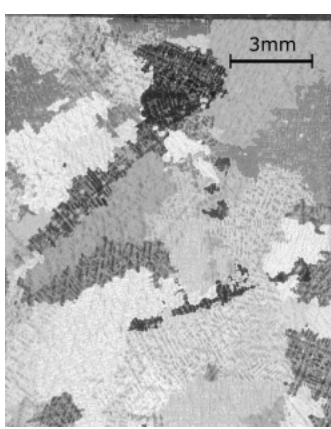

(b)

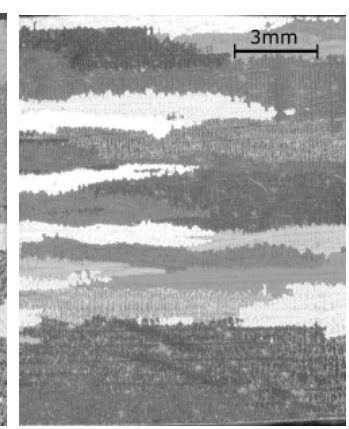

(c)

Figure 3.18: Microstructure of MAR-M247 DS alloy. The images (a) and (b) show the cross-section perpendicular to grain-growth direction and the image (c) in the parallel direction. 
The grain-size distribution (Figure 3.19a) was analysed by using the opensource software ImageJ [89]. Each grain was fitted to an ellipse with the same area and orientation, and the characteristic length was calculated as $0.5(a+b)$, where $a$ and $b$ stands for the major and minor axis of the ellipse respectively (Figure 3.19b). The analysis of 25 grains showed a size range from one to seven millimetres in the cross-section perpendicular to the grain-growth direction. The grain-size data were fitted with a log-normal distribution, getting the values $d_{g}=1.67 \ln (\mathrm{mm})$ and $d_{S D}=0.073 \ln (\mathrm{mm})$, which are the mean and standard deviation of the variables' natural logarithm. A mean grain size of $5.5 \mathrm{~mm}$ was obtained.

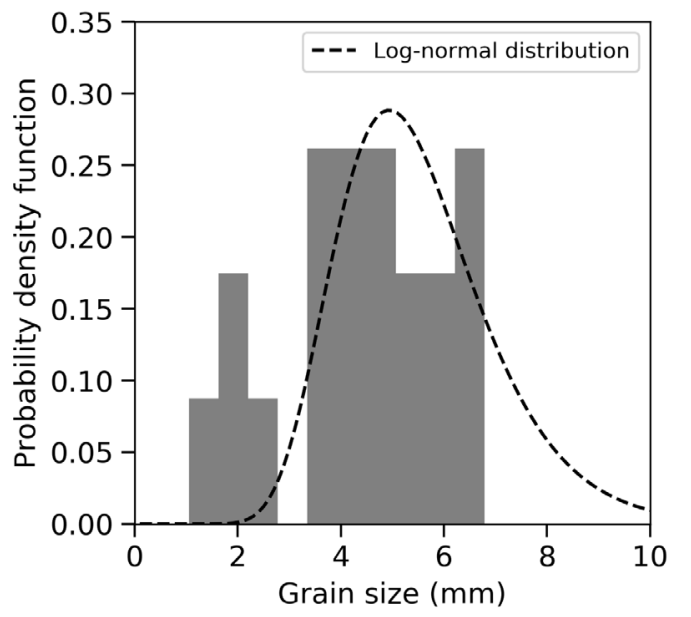

(a)

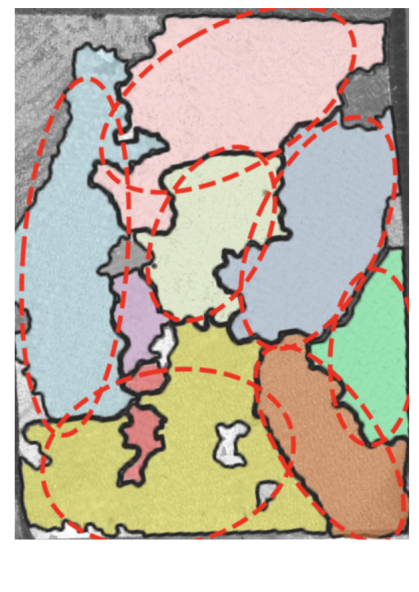

(b)

Figure 3.19: (a) Grain-size distribution of MAR-M247 DS alloy and (b) ellipse fitting of the grains to estimate the characteristic length $d_{g}$.

\subsubsection{Experiments}

The behaviour of the alloy was characterised by means of quasi-static and high-strain-rate $\left(150 \mathrm{~s}^{-1}\right.$ and $\left.500 \mathrm{~s}^{-1}\right)$ uniaxial tensile tests on axisymmetric specimens obtained parallel $\left(0^{\circ}\right)$ and perpendicularly $\left(90^{\circ}\right)$ to grain-growth direction. These two orientations were chosen to obtain information about the anisotropic behaviour. The tests at different strain rates were used to calculate the strain-rate sensitivity, but the yield-strength anomaly was only studied 
through $500 \mathrm{~s}^{-1}$ tests. The geometry and dimensions of the specimens can be seen in Figure 3.20. The sample (a) was used in the tests performed at $2.5 \cdot 10^{-4} \mathrm{~s}^{-1}$ and $500 \mathrm{~s}^{-1}$, while the sample (b) was employed in the tests at $150 \mathrm{~s}^{-1}$ because of limitations of the experimental device.

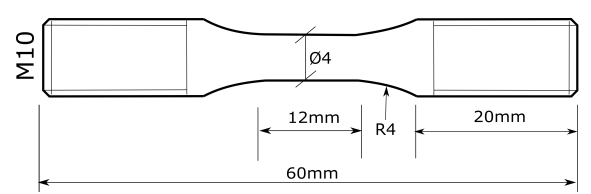

(a)

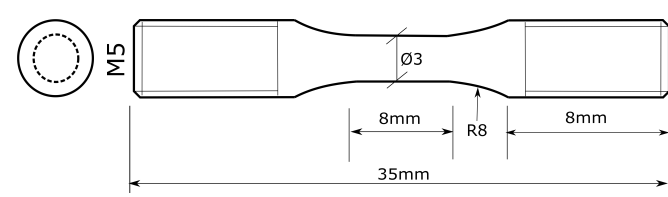

(b)

Figure 3.20: Geometry and dimensions of the smooth tensile samples employed in (a) quasi-static and $500 \mathrm{~s}^{-1}$ tests and (b) $150 \mathrm{~s}^{-1}$ tests.

\section{Quasi-static tensile tests}

Six quasi-static tensile tests were carried out at room temperature, three along the $0^{\circ}$ direction and three along the $90^{\circ}$ direction. The tests were performed in an Instron servo-hydraulic universal testing machine at an engineering strain rate of $\dot{e}=2.5 \cdot 10^{-4} \mathrm{~s}^{-1}$. Each test was recorded with a video camera at two frames per second and the edge-tracing technique, implemented in the eCorr software [90], was employed to monitor the evolution the cross-section diameter of the specimens (Figure 3.21). Then, the mechanical response of the alloy was calculated as:

$$
\begin{gathered}
\sigma=\frac{4 F}{\pi D^{2}} \\
\varepsilon=2 \ln \left(\frac{D_{0}}{D}\right)
\end{gathered}
$$

where $F$ is the force and $D_{0}$ the initial cross-section diameter.

The true stress-strain curves are plotted in Figure 3.22. It is important to note that both axes were non-dimensionalised for reasons of confidentiality. As expected, the alloy displayed a clear anisotropic behaviour, which is triggered by the texture developed by the material due to the directional-solidification processing technique. The reason why the DS microstructure leads to a higher 


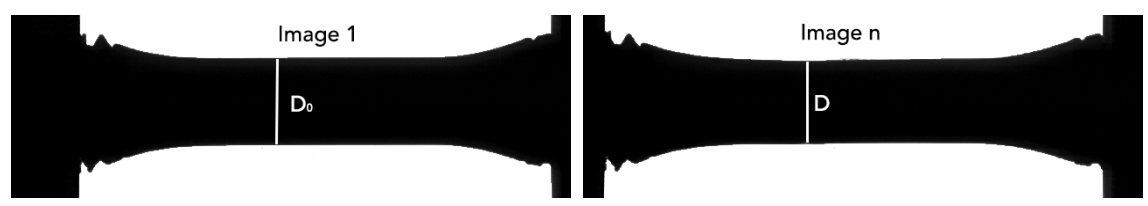

(a)

(b)

Figure 3.21: Edge-tracing technique. (a) Initial frame and (b) frame just before failure.

performance in the $0^{\circ}$ direction will be discussed at length, using the crystalplasticity finite element method, in Chapter 4. Despite the big size of the grains, the experimental scatter was minimum with regard to the flow behaviour, but it was important when it comes to the failure strain. However, failure is not of interest in the present section.

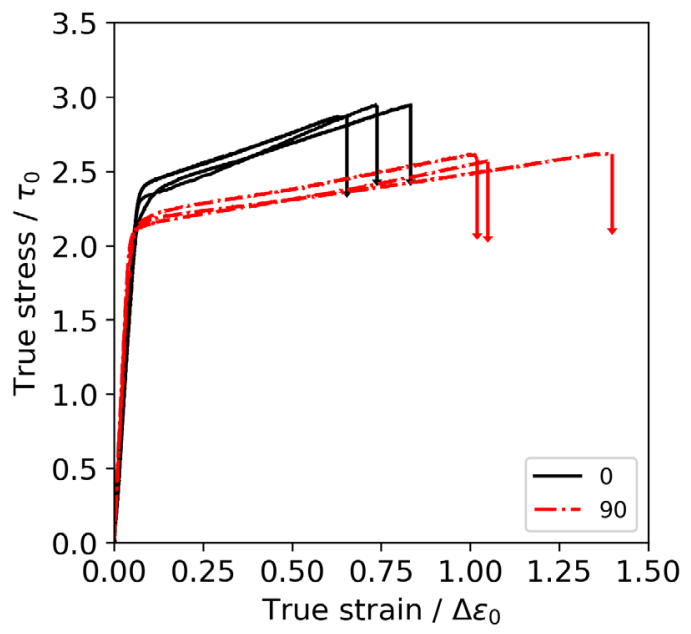

Figure 3.22: Quasi-static true stress-strain curves of MAR-M247 DS alloy along $0^{\circ}$ and $90^{\circ}$ direction. $\tau_{0}$ and $\Delta \varepsilon_{0}$ are confidential values suggested ITP Aero company.

\section{Dynamic tensile tests}

Dynamic tensile tests were carried out by using the Split-Hopkinson tension bar technique (SHTB). Two different configurations were used because the tests at $150 \mathrm{~s}^{-1}$ strain rate were done in the Structural Impact Laboratory (SIMLab) at Norwegian University of Science and Technology, while the tests at $500 \mathrm{~s}^{-1}$ 
were performed in the Materials Science Department at Universidad Politécnica de Madrid (UPM).

The SHTB of SIMLab, depicted in Figure 3.23 and explained in detail in [91], consists of two 10-mm-diameter steel bars. In this configuration, the incident bar is clamped in the point $B$ and the stress wave is generated by pre-stretching the segment $\overline{\mathrm{AB}}$ with a force $N_{0}$ (measured with the strain gauge 3 ) and then, releasing the clamp. The released stress wave, with magnitude $0.5 \mathrm{~N}_{0} / \mathrm{A}_{\mathrm{b}}$ and length $2 \overline{\mathrm{AB}}$, moves forward until the point $\mathrm{C}$, where it is partly reflected and partly transmitted into the second bar. Using the information from the strain gauges 1 and 2, the stress-strain curves were calculated according to the splitHopkinson bar theory (Section 2.5). In the present case, the tests were recorded at 150000 fps and $640 \times 208$ px resolution by using a Phantom V2511 high-speed camera (Figure 3.24). Then, the strain was calculated from the cross-sectiondiameter evolution (equation 3.26) obtained after analysing the images with the eCorr software.

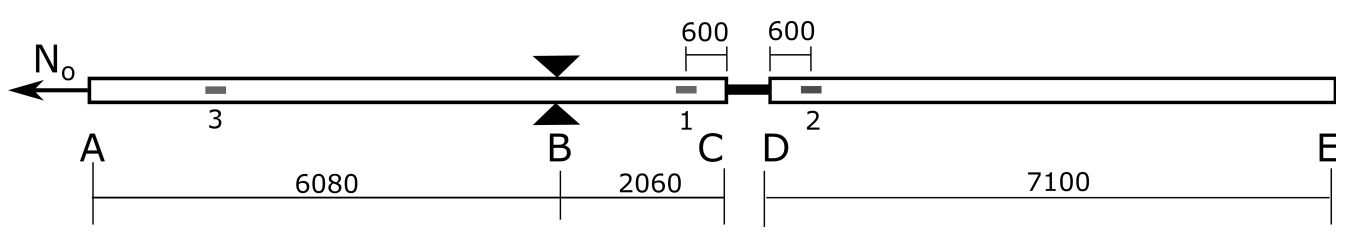

Figure 3.23: Split-Hopkinson tension bar for tests at $150 \mathrm{~s}^{-1}$ (SIMLab). The strain gauges are in positions 1,2 and 3.

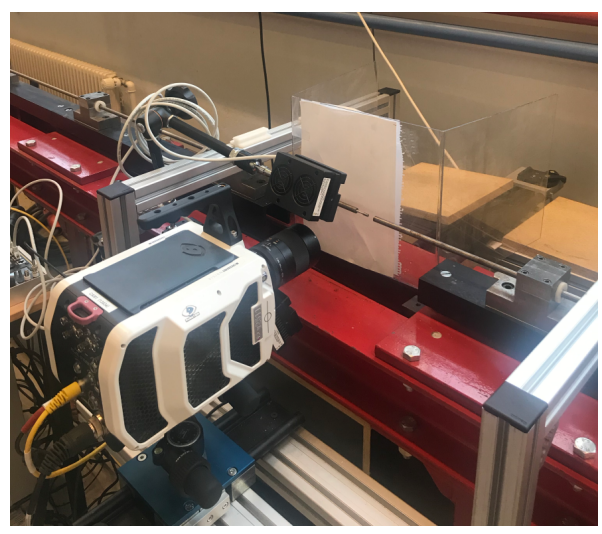

Figure 3.24: Experimental set-up for tests at $150 \mathrm{~s}^{-1}$ strain rate with the high-speed camera. 
Dynamic tests at $500 \mathrm{~s}^{-1}$ strain rate from ambient to $1073 \mathrm{~K}$ were performed with the SHTB located in the Materials Science department at UPM. The device, which details are given in Figure 3.25 consists of a 400-mm-long projectile, an incident and transmission bars, both $1500 \mathrm{~mm}$ long, made of René-41 alloy. The incident tensile pulse is created by launching, using compressed air, the striker bar against a flange connected to the end of the incident bar. Once the pulse is generated, it travels along the input bar towards the bar/specimen interface and the test continuous as described in Section 2.5. In this case, the tests were not recorded and the strain history was obtained from the bar signals.

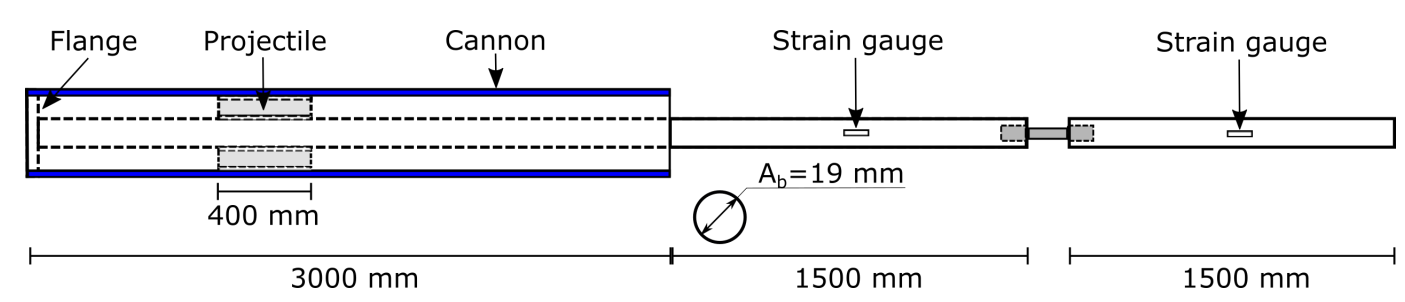

Figure 3.25: Split-Hopkinson Tension Bar set-up for tests at $500 \mathrm{~s}^{-1}$ (UPM).

The high temperature tests were performed by mounting the same $\mathrm{SiC}$ resistor furnace as that used for the SHPB tests (see Figure 3.3 in Section 3.2), where a thermocouple in contact with the specimen defines the temperature of the test. The effect of temperature on the stress-waves propagation was disregarded when analysing the signals from high-temperature tests. The reason was already justified in Section 3.2.

Figure 3.26 exhibits the response of the material at $150 \mathrm{~s}^{-1}$ and room temperature. The anisotropic behaviour remains and a low strain-rate sensitivity is observed for both directions when comparing with the quasi-static results. However, the experimental scatter increases, specially for $90^{\circ}$ tests.

Figure 3.27 collects the true stress-strain response obtained at $500 \mathrm{~s}^{-1}$ for different temperatures $(298 \mathrm{~K}, 573 \mathrm{~K}, 700 \mathrm{~K}, 823 \mathrm{~K}, 948 \mathrm{~K}$ and $1073 \mathrm{~K})$ in $0^{\circ}$ and $90^{\circ}$ orientations. Three tests, $0^{\circ}$ at $1073 \mathrm{~K}$ and $90^{\circ}$ at $573 \mathrm{~K}$ and $823 \mathrm{~K}$, were rejected due to premature failure during loading. Unlike tests at $150 \mathrm{~s}^{-1}$, the curves show some oscillations at the beginning of yielding as a consequence of the combination of the Pochhammer-Chree effect and the wave reflections in the 


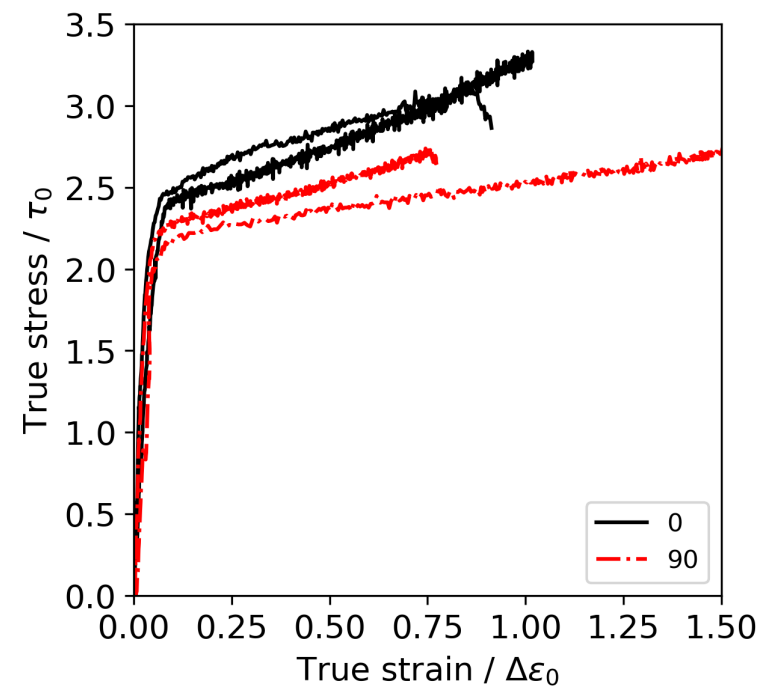

Figure 3.26: Dynamic true stress-strain curves of MAR-M247 DS alloy along $0^{\circ}$ and $90^{\circ}$ direction at an engineering strain rate of $150 \mathrm{~s}^{-1}$ and room temperature. $\tau_{0}$ and $\Delta \varepsilon_{\max }$ are confidential values suggested ITP Aero company.

screwed-threaded union between specimen and bars. At high temperatures, the magnitude of the oscillations increases due to the different thermal-expansion coefficient between materials.

Analysing the curves at $298 \mathrm{~K}$ and comparing with the above results for lower strain rates, the low strain-rate sensitivity of the alloy was confirmed (see Table 3.8). This behaviour is based on its microstructure, an FCC matrix hardened with a high volume fraction of precipitates, i.e. long-range obstacles that cannot be overcome by thermal activation.

Table 3.8: $0.2 \%$ yield strength of MAR-M247 at testing strain rates and room temperature.

\begin{tabular}{ccc}
\hline Strain rate & $\sigma_{0.2}\left(0^{\circ}\right)$ & $\sigma_{0.2}\left(90^{\circ}\right)$ \\
\hline $2.5 \cdot 10^{-4} \mathrm{~s}^{-1}$ & $2.41 \tau_{0} \pm 0.02 \tau_{0}$ & $2.18 \tau_{0} \pm 0.01 \tau_{0}$ \\
$150 \mathrm{~s}^{-1}$ & $2.44 \tau_{0} \pm 0.04 \tau_{0}$ & $2.24 \tau_{0} \pm 0.04 \tau_{0}$ \\
$500 \mathrm{~s}^{-1}$ & $2.47 \tau_{0} \pm 0.01 \tau_{0}$ & $2.25 \tau_{0} \pm 0.01 \tau_{0}$ \\
\hline
\end{tabular}




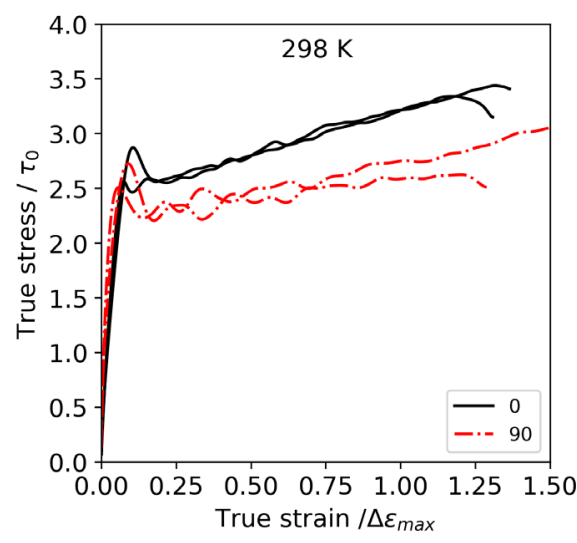

(a)

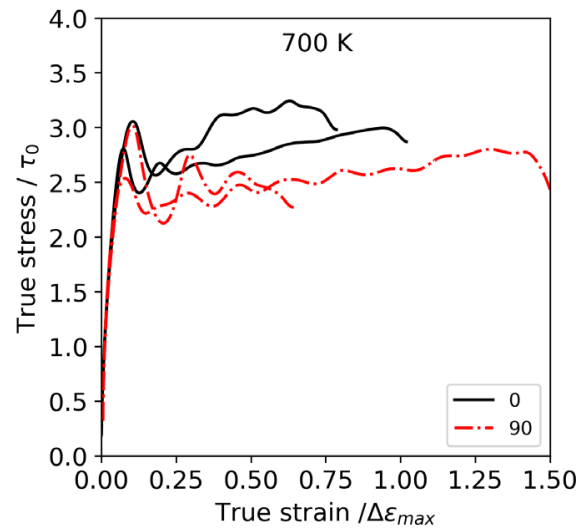

(c)

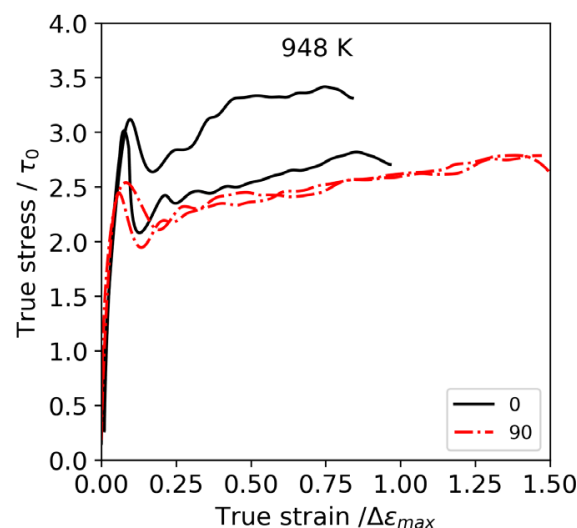

(e)

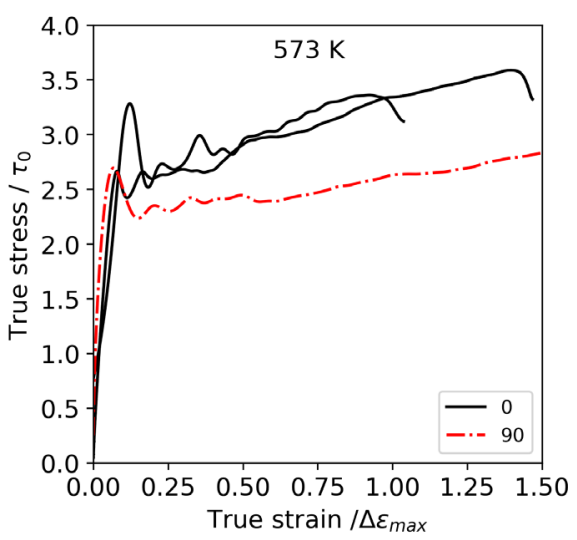

(b)

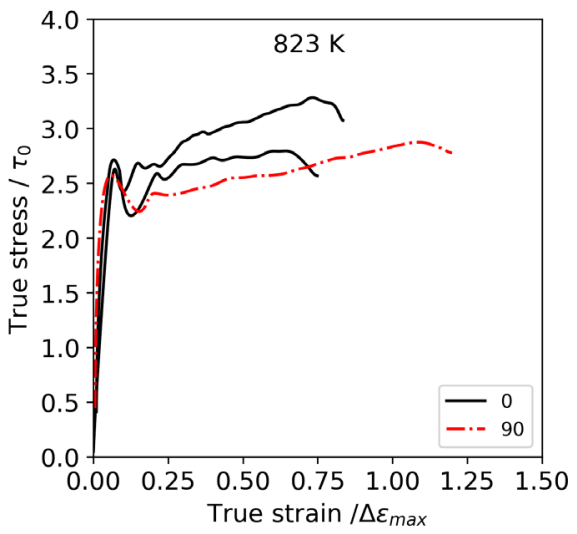

(d)

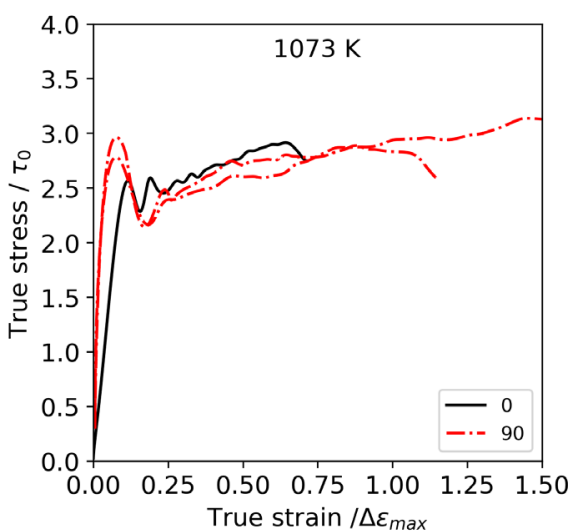

(f)

Figure 3.27: Experimental true stress-strain curves of the dynamic tests $\left(500 \mathrm{~s}^{-1}\right)$ at different temperatures for $0^{\circ}$ and $90^{\circ}$ orientations. $\tau_{0}$ and $\Delta \varepsilon_{\max }$ are confidential values suggested by ITP Aero company. $\Delta \varepsilon_{\max }=\Delta \varepsilon_{0}$. 
Regarding the temperature dependence of the yield strength, the material exhibits low sensitivity up to 1073 K. Figure 3.28 plots the yield strength evolution in more detail. From ambient to $948 \mathrm{~K}$, the temperature dependence is slightly positive, with a $3 \%$ increase at $823 \mathrm{~K}$ in the $0^{\circ}$ orientation and a $4 \%$ increase along the $90^{\circ}$. At $948 \mathrm{~K}$, the yield strength values tend to decrease although there is an important uncertainty from $0^{\circ}$ data. In the final part of the curve, at $1073 \mathrm{~K}$, the softening behaviour appears in $0^{\circ}$ samples while $90^{\circ}$ samples keeps a strength similar to that at room temperature. Despite these differences, assuming the same yield strength evolution (solid line) is accurate enough and able to capture the trend of experimental data, obtained quasi-statically, from the same material but with equiaxed grains (diamond markers) [92].

Figure 3.28 was obtained, as in previous cases, by setting the flow stress at room temperature as reference $\left(\sigma_{r e f}\right)$ and scaling the experimental curves for the rest of temperatures until the difference of the flow stress in the $0-0.3 \Delta \varepsilon_{\max }$ strain range was minimized. Since some experiments showed an important uncertainty, the mean value was considered. Finally, it is important to note that $\sigma_{\text {ref }}$ takes also into account the anisotropic ratio between the parallel and perpendicular orientation. Moreover, the data from M. Kaufman [92] was scaled down for the sake of legibility.

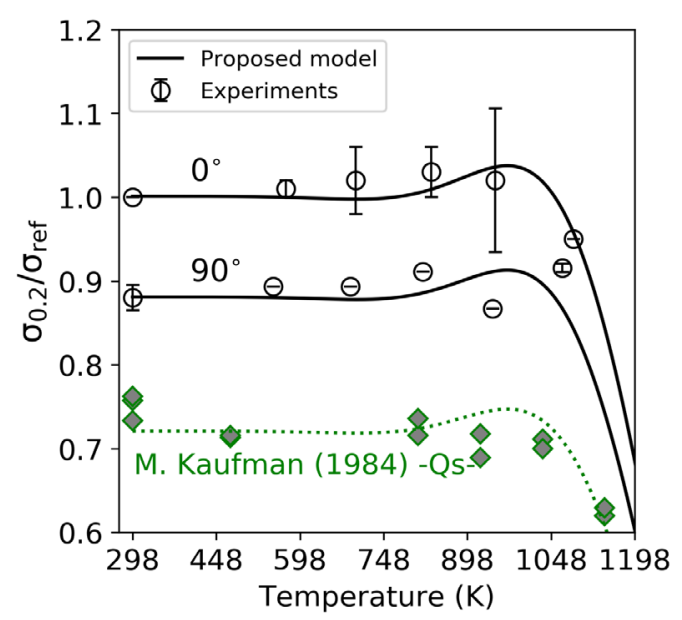

Figure 3.28: Temperature dependence of MAR-M247 yield strength along $0^{\circ}$ and $90^{\circ}$ direction. Markers depict the mean value with the error bar. 


\subsubsection{Numerical simulations}

\section{Parameter identification}

Despite the anisotropic behaviour of the DS superalloy, the isotropic model described at the beginning of the chapter was employed to model the behaviour of the superalloy and the flow-stress difference between $0^{\circ}$ and $90^{\circ}$ directions was considered by setting two different values for the yield strength constant $A$. This decision was made on the basis that the main goal of this chapter is to check the capability of the proposed model to deal with the yield-strength anomaly under dynamic loading and not to simulate anisotropic yielding. This kind of behaviour, due to the heterogeneous grain structure of the material, will be analysed in the next chapter.

The parameter-identification procedure was similar to that described in previous sections. All material constants are gathered in Table 3.9. The constants that define the temperature dependence of flow stress $\left(T_{m}, m_{t}, Y_{p 0}, w_{p}, n\right)$ were adjusted by using the experimental data of the temperature dependence of the yield strength depicted in Figure 3.28. Both directions $\left(0^{\circ}\right.$ and $\left.90^{\circ}\right)$ were considered during fitting. The yield strength $(A)$, the parameters of the hardening law $\left(Q_{1}, C_{1}\right)$ and the Young's modulus $(E)$ were obtained from the quasi-static true stress-strain curves. Two different yield strength constants $\left(A_{0}, A_{90}\right)$ were set depending on sample orientation, while hardening was assumed independent on loading direction. The Young's modulus $\left(E=452 \tau_{0}\right)$ was also assumed isotropic and its value was calculated as the mean of $E_{0}$ and $E_{90}$. Besides, the Poisson's ratio was set equal to 0.3 . The strain rate sensitivity parameter $k=0.0018$ (for $\dot{\varepsilon}_{0}=2.5 \cdot 10^{-4} s^{-1}$ ) was determined from the $0.2 \%$ yield strength of MAR-M247 at room temperature for the different strain rates (Table 3.8).

Finally, with respect to the constants that control the adiabatic heating, a density value $\rho=8540 \mathrm{~kg} / \mathrm{m}^{3}$ was reported by the material supplier. No information was found about the specific heat capacity and the Taylor-Quinney coefficient. Therefore, the pure-nickel value $C_{p}=440 \mathrm{~J} / \mathrm{kg} / \mathrm{K}$ and the standard Taylor-Quinney coefficient $\beta=0.9$ were assumed. 
Table 3.9: Material constants for MAR-M247.

\begin{tabular}{ccccccccc}
\hline$A_{0} / / A_{90}$ & $Q_{1}$ & $C_{1}$ & $T_{m}(\mathrm{~K})$ & $m_{t}(-)$ & $Y_{p 0}$ & $T_{p}(\mathrm{~K})$ & $w_{p}(\mathrm{~K})$ & $n(-)$ \\
\hline $2.43 / / 2.18 \tau_{0}$ & $7.06 \tau_{0}$ & 1 & 1374 & 5.0 & $0.507 \tau_{0}$ & 1088 & 208.5 & 1.0 \\
\hline
\end{tabular}

\section{Simulations}

The dynamic tests $\left(500 \mathrm{~s}^{-1}\right)$ at different temperatures were simulated by using the Abaqus FEA code. Quasi-static and dynamic tests at $150 \mathrm{~s}^{-1}$ were not simulated here but in the next chapter. The dimensions and geometry of the virtual specimen were the same as those for the experimental tests, but due to the symmetry of the model, only one fourth of the specimen was considered (Figure 3.29). The sample was meshed using eight-node solid elements with reduced integration (C3D8R) and default hourglass control. The approximate element size in the gauge section was $0.2 \times 0.2 \times 0.1 \mathrm{~mm}^{3}$, being the smaller dimension parallel to the loading direction.

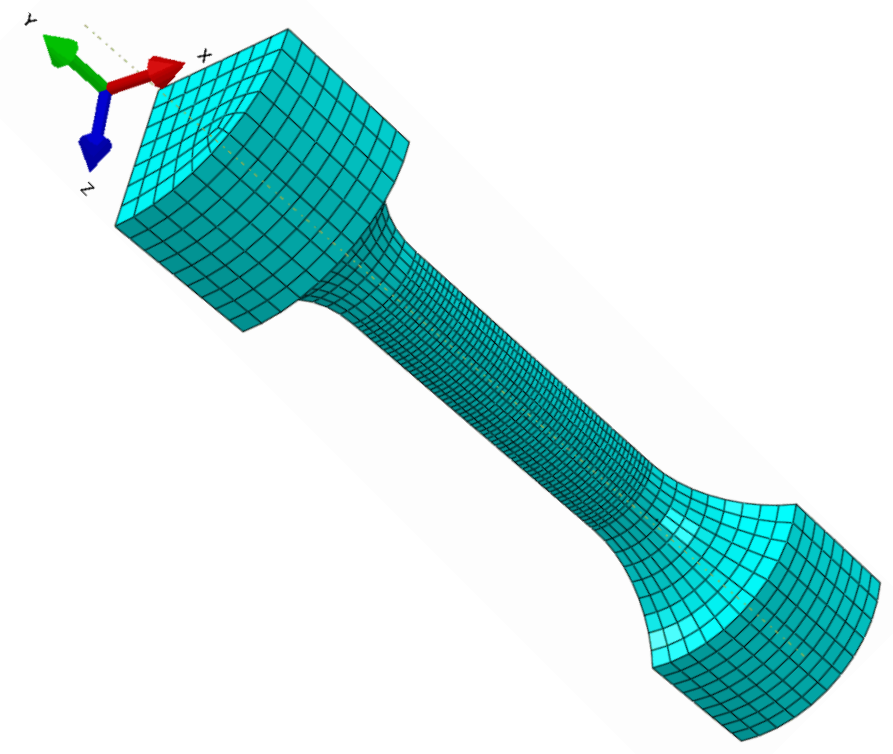

Figure 3.29: Finite element model used for numerical simulation of MARM247 under dynamic loading and different temperatures. 
Boundary conditions to replicate the SHTB test were applied directly on the sample ends. The displacement of the lower end was constrained in the $\mathrm{y}$-direction while the velocity profile described in equation 3.27 was imposed to nodes on the upper end. Symmetry boundary conditions were applied to the nodes on the symmetry planes $x=0$ and $z=0$ (equation 3.28). The simulations were run with the explicit solver by using the default time step calculated by Abaqus.

$$
v_{y=L}=\left\{\begin{array}{l}
250 \cdot t \text { for } t \leq 0.024 \mathrm{~ms} \\
6 \text { for } t>0.024 \mathrm{~ms}
\end{array} \mathrm{~mm} / \mathrm{ms}\right.
$$

$$
\begin{aligned}
& \text { Symmetry about plane } x=0 \rightarrow u_{x}=u_{R_{y}}=u_{R_{z}}=0 \\
& \text { Symmetry about plane } z=0 \rightarrow u_{z}=u_{R_{x}}=u_{R_{y}}=0
\end{aligned}
$$

Finally, the mechanical response given by the simulations was computed from the reaction forces and displacement of the nodes on the end $y=L$. Figure 3.30 shows the comparison between numerical and experimental results. In general, there is good agreement for all temperature ranges although the softening, due to adiabatic heating, predicted by the simulations overestimates the experimental observations at $1073 \mathrm{~K}$. 


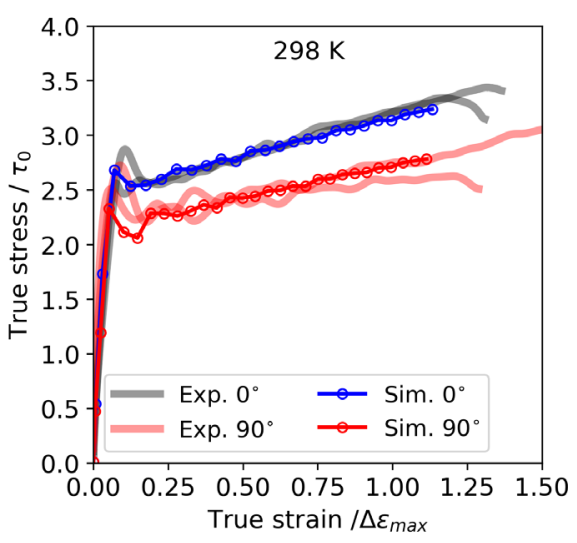

(a)

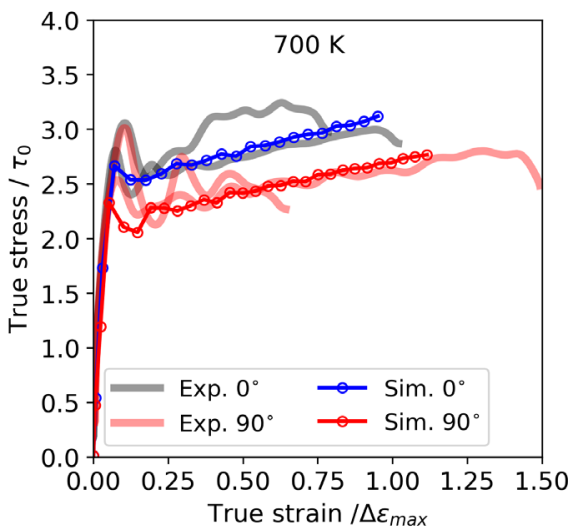

(c)

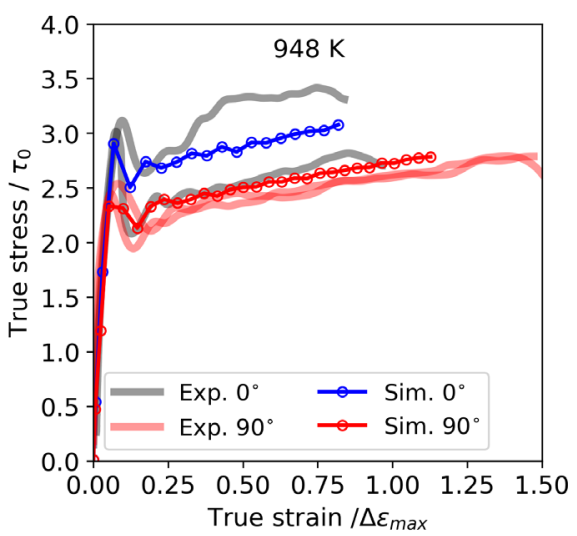

(e)

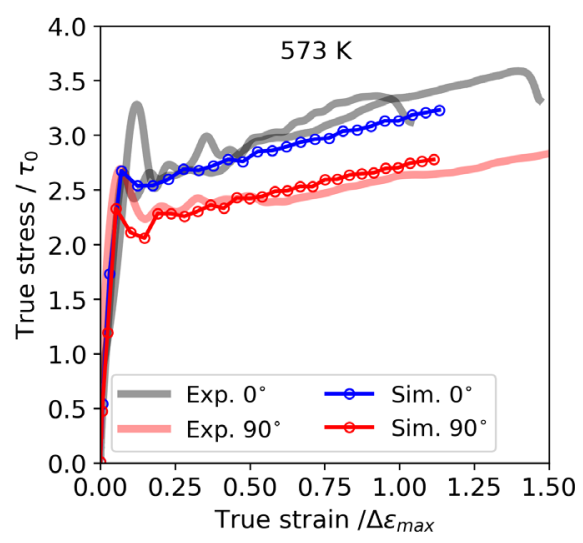

(b)

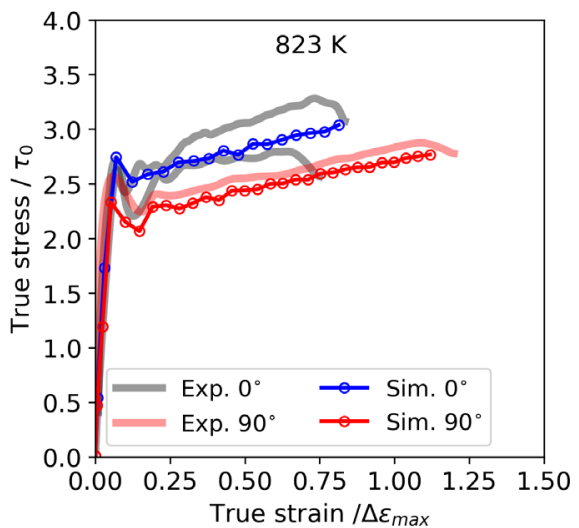

(d)

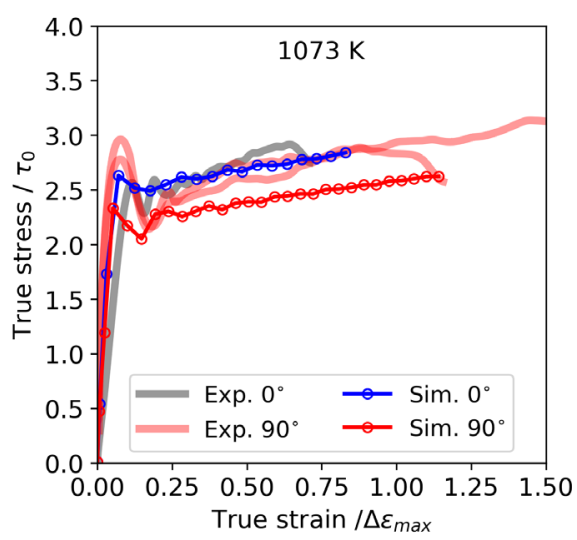

(f)

Figure 3.30: Numerical true stress-strain curves compared with the experimental data for dynamic tensile tests $\left(500 \mathrm{~s}^{-1}\right)$ at different temperatures. $\tau_{0}$ and $\Delta \varepsilon_{\max }$ are confidential values suggested by ITP Aero company. 
Chapter 4

\section{CP modelling of MAR-M247 DS}

This chapter deals with the analysis, through CPFEM technique, of the deformation behaviour of MAR-M247 DS alloy at quasi-static and high strain rates presented in Section 3.4. The crystal response (Section 4.1) is modelled using a standard visco-plastic approach, in which viscous slip is described with a power law and the evolution of the critical resolved shear stress is considered through a phenomenological hardening model with strength-interaction-matrix coefficients taken from lower-scale simulations. The numerical strategy (Section 4.2) accounts for the explicit representation of the grain characteristics (size, aspect ratio and orientation) along the specimen gauge length, based on the experimentally grain-size analysis. The parameters of the hardening law and viscous response were obtained from experiments by using an inverse analysis of the experimental tests in $0^{\circ}$ direction (Section 4.3). Independent experimental results were employed to validate the predictive capability of the model (Section 4.4). Moreover, the simulations were used to analyse the stress micro-fields for each configuration and their influence on the failure behaviour.

\subsection{Crystal plasticity model}

In this section, the crystal plasticity model used to simulate the behaviour of the alloy is presented. The model was formulated and implemented, following 
the strategy described in [39], in the commercial finite-element code Abaqus through a UMAT user-material subroutine [66].

The model assumes that the deformation gradient $\mathbf{F}$ can be decomposed multiplicatively into elastic $\boldsymbol{F}^{\boldsymbol{e}}$ and plastic $\boldsymbol{F}^{\boldsymbol{p}}$ parts according to [32]:

$$
\mathbf{F}=\mathbf{F}^{\mathrm{e}} \mathbf{F}^{\mathbf{p}}
$$

Using the definition of the velocity gradient $\mathbf{L} \equiv \nabla_{\mathbf{x}} \mathbf{v}=\dot{\mathbf{F}} \mathbf{F}^{-\mathbf{1}}$, the expression 4.1 leads to the additive decomposition of $\mathbf{L}$ as:

$$
\mathbf{L}=\mathbf{L}^{\mathrm{e}}+\mathbf{F}^{\mathrm{e}} \mathbf{L}^{\mathbf{p}}\left(\mathbf{F}^{\mathrm{e}}\right)^{-1}
$$

Since plastic deformation in a crystal takes place along different slip systems $\alpha$, the plastic-deformation velocity gradient is defined as:

$$
\mathbf{L}^{\mathbf{p}}=\sum_{\alpha} \dot{\gamma}^{\alpha}\left(\mathbf{s}^{\alpha} \otimes \mathbf{m}^{\alpha}\right)
$$

where $\dot{\gamma}^{\alpha}$ is the plastic-slip rate on the slip system $\alpha$ and $\mathbf{s}^{\alpha}$ and $\mathbf{m}^{\alpha}$ stand, respectively, for the unit vectors in the slip direction and normal to the slip plane in the reference configuration.

In nickel-base superalloys, deformation takes place due to the contributions of octahedral (FCC matrix $+\mathrm{L}_{2}$ precipitates) and cube ( $\mathrm{L}_{2}$ precipitates) slip systems [51, 93, 44]. However, the importance of the different deformation mechanisms depends on temperature. It is well accepted that at low temperatures $\left(\mathrm{T}<<\mathrm{T}_{\text {peak }}\right)$, in Ni-base superalloys, most dislocations lie in the matrix and those that shear the precipitates move also on the $\gamma^{\prime}$ octahedral planes with low rate of cross-slip. Furthermore, it is not until higher temperatures, above $600 \mathrm{~K}$ according to $[51,44]$, when cube slip in $\gamma^{\prime}$ phase activates. Therefore, in the present work, the material behaviour is described as a crystal with $12\{111\}<110>$ slip systems since only room temperature is considered (Table 4.1). This approximation implies that the CRSS and hardening behaviour of the octahedral systems accounts for the strengthening effect of the $\gamma^{\prime}$ precipitates. The approach has been used and considered accurate enough by other authors [94, 22] for small amounts of strain. Nevertheless, its accuracy is also demonstrated for larger strains throughout this chapter. 
Table 4.1: Octahedral slip systems.

\begin{tabular}{ccccc}
\hline System ID & $\mathbf{m}^{\alpha}$ & $\mathbf{s}^{\alpha}$ \\
\hline$S_{1}$ & $(111)$ & {$[1 \overline{1} 0]$} \\
$S_{2}$ & $(111)$ & {$[0 \overline{1} 1]$} \\
$S_{3}$ & $(111)$ & {$[\overline{1} 01]$} \\
$S_{4}$ & $(1 \overline{1} 1)$ & {$[110]$} \\
$S_{5}$ & $(1 \overline{1} 1)$ & {$[011]$} \\
$S_{6}$ & $(1 \overline{1} 1)$ & {$[\overline{1} 01]$} \\
$S_{7}$ & $(\overline{111})$ & {$[1 \overline{1} 0]$} \\
$S_{8}$ & $(\overline{111})$ & {$[011]$} \\
$S_{9}$ & $(\overline{11} 1)$ & {$[101]$} \\
$S_{10}$ & $(\overline{1} 11)$ & {$[110]$} \\
$S_{11}$ & $(\overline{1} 11)$ & {$[0 \overline{1} 1]$} \\
$S_{12}$ & $(\overline{1} 11)$ & {$[101]$}
\end{tabular}

The crystal is assumed to behave as an elasto-viscoplastic solid, in which the plastic-slip rate follow the power law [95]:

$$
\dot{\gamma}^{\alpha}=\dot{\gamma}_{0}\left(\frac{\left|\tau^{\alpha}\right|}{g^{\alpha}}\right)^{1 / m} \operatorname{sign}\left(\tau^{\alpha}\right)
$$

where $\dot{\gamma}_{0}$ is the reference shear-strain rate, $g^{\alpha}$ the critical resolved shear stress (CRSS), $\tau^{\alpha}$ the resolved shear stress and $m$ the rate-sensitivity exponent. The resolved shear stress $\tau$ on the slip system $\alpha$ is obtained by the projection of the second Piola-Kirchhoff stress tensor $\mathbf{S}$ on the corresponding slip system as:

$$
\tau^{\alpha}=\mathbf{S}:\left(\mathbf{s}^{\alpha} \otimes \mathbf{m}^{\alpha}\right)
$$

The second Piola-Kirchhoff stress tensor $\mathbf{S}$ is obtained by applying the double inner product between the fourth order elastic stiffness tensor $\mathbb{C}$ and GreenLagrange strain tensor $\mathbf{E}^{\mathbf{e}}$ :

$$
\mathbf{S}=\mathbb{C}: \mathbf{E}^{\mathbf{e}}
$$


The evolution of the critical resolved shear stress (CRSS), $g^{\alpha}$, for a given slip system $\alpha$, is expressed as:

$$
\dot{g}^{\alpha}=\sum_{\beta=1}^{12} \mathrm{Q}^{\alpha \beta} h(\Gamma) \dot{\gamma}^{\beta}
$$

where $\mathbf{Q}^{\alpha \beta}$ is a $12 \times 12$ symmetric matrix that describes, with six independent constants $\left(q_{1} \ldots q_{6}\right)$ in the case of FCC materials, the strength of the different interactions between pairs of slip systems [96]. The interactions are collected in detail in Table 4.2. The first three terms $\left(q_{1}, q_{2}\right.$ and $\left.q_{3}\right)$ account, respectively, for self-interaction of dislocations in the same slip system, interaction of coplanar dislocations (same plane but different Burgers vector) and interaction between collinear dislocations (same Burgers vector but different plane). The remaining terms $\left(q_{4}, q_{5}\right.$ and $\left.q_{6}\right)$ have to do with dislocation junctions (Hirth lock, glissile junction and Lomer-Cottrell lock) [96]. The magnitude of the interaction coefficients can be calculated by lower-scale simulations, i.e, discrete dislocation dynamics.

Table 4.2: Coefficients of the strength-interaction matrix $\mathbf{Q}^{\alpha \beta}$ for an FCC material. Each system ID is defined in Table 4.1

\begin{tabular}{|c|c|c|c|c|c|c|c|c|c|c|c|c|}
\hline System & $S_{1}$ & $S_{2}$ & $S_{3}$ & $S_{4}$ & $S_{5}$ & $S_{6}$ & $S_{7}$ & $S_{8}$ & $S_{9}$ & $S_{10}$ & $S_{11}$ & $S_{12}$ \\
\hline$S_{1}$ & $\mathrm{q}_{1}$ & $\mathrm{q}_{2}$ & $\mathrm{q}_{2}$ & $\mathrm{q}_{4}$ & $\mathrm{q}_{6}$ & $\mathrm{q}_{5}$ & $\mathrm{q}_{3}$ & $\mathrm{q}_{5}$ & $\mathrm{q}_{5}$ & $\mathrm{q}_{4}$ & $\mathrm{q}_{5}$ & $\mathrm{q}_{6}$ \\
\hline$S_{2}$ & & $\mathrm{q}_{1}$ & $\mathrm{q}_{2}$ & $\mathrm{q}_{6}$ & $\mathrm{q}_{4}$ & $\mathrm{q}_{5}$ & $\mathrm{q}_{5}$ & $\mathrm{q}_{4}$ & $\mathrm{q}_{6}$ & $q_{5}$ & $\mathrm{q}_{3}$ & $\mathrm{q}_{5}$ \\
\hline$S_{3}$ & & & $q_{1}$ & $\mathrm{q}_{5}$ & $\mathrm{q}_{5}$ & $\mathrm{q}_{3}$ & $\mathrm{q}_{5}$ & $\mathrm{q}_{6}$ & $\mathrm{q}_{4}$ & $\mathrm{q}_{6}$ & $\mathrm{q}_{5}$ & $\mathrm{q}_{4}$ \\
\hline$S_{4}$ & & & & $\mathrm{q}_{1}$ & $\mathrm{q}_{2}$ & $\mathrm{q}_{2}$ & $\mathrm{q}_{4}$ & $\mathrm{q}_{5}$ & $\mathrm{q}_{6}$ & $\mathrm{q}_{3}$ & $\mathrm{q}_{5}$ & $\mathrm{q}_{5}$ \\
\hline$S_{5}$ & & & & & $\mathrm{q}_{1}$ & $\mathrm{q}_{2}$ & $\mathrm{q}_{5}$ & $\mathrm{q}_{3}$ & $\mathrm{q}_{5}$ & $\mathrm{q}_{5}$ & $\mathrm{q}_{4}$ & $\mathrm{q}_{6}$ \\
\hline$S_{6}$ & & & & & & $\mathrm{q}_{1}$ & $\mathrm{q}_{6}$ & $\mathrm{q}_{5}$ & $\mathrm{q}_{4}$ & $\mathrm{q}_{5}$ & $\mathrm{q}_{6}$ & $\mathrm{q}_{4}$ \\
\hline$S_{7}$ & & & symm & & & & $\mathrm{q}_{1}$ & $\mathrm{q}_{2}$ & $\mathrm{q}_{2}$ & $\mathrm{q}_{4}$ & $\mathrm{q}_{6}$ & $\mathrm{q}_{5}$ \\
\hline$S_{8}$ & & & & & & & & $\mathrm{q}_{1}$ & $\mathrm{q}_{2}$ & $\mathrm{q}_{6}$ & $\mathrm{q}_{4}$ & $\mathrm{q}_{5}$ \\
\hline$S_{9}$ & & & & & & & & & $\mathrm{q}_{1}$ & $\mathrm{q}_{5}$ & $\mathrm{q}_{5}$ & $\mathrm{q}_{3}$ \\
\hline$S_{10}$ & & & & & & & & & & $\mathrm{q}_{1}$ & $\mathrm{q}_{2}$ & $\mathrm{q}_{2}$ \\
\hline$S_{11}$ & & & & & & & & & & & $\mathrm{q}_{1}$ & $\mathrm{q}_{2}$ \\
\hline$S_{12}$ & & & & & & & & & & & & $\mathrm{q}_{1}$ \\
\hline
\end{tabular}


Since the studied alloy shows a quasi-linear plastic behaviour, the hardening law proposed by Assaro-Needleman [95, 97] was used due to its low number of constants. The function reads:

$$
h(\Gamma)=h_{0} \operatorname{sech}^{2}\left(\frac{h_{0} \Gamma}{\tau_{s}-\tau_{0}}\right)
$$

where $h_{0}$ is the initial hardening modulus and $\tau_{0}$ and $\tau_{s}$ stand for the initial and the saturation values of the CRSS, respectively. $\Gamma$ is the accumulated plastic slip on all the slip systems formulated as:

$$
\Gamma=\sum_{\alpha}\left|\dot{\gamma}^{\alpha}\right| d t
$$

The model does not consider the effect of temperature because all mechanical tests were performed at $298 \mathrm{~K}$ and the effect of adiabatic heating can be disregarded since the yield strength of the alloy is temperature independent until $800 \mathrm{~K}$, as it was discussed in the previous chapter (Figure 3.28).

\subsubsection{Time discretization}

The non-linear finite-element problem is linearised through applying the boundary conditions as a function of time and discretising the total time with time increments $\Delta t$. Considering the time interval $t_{n}-t_{n+1}$, the stress update consists in solving the next set of equations (4.10 - 4.16)[28], knowing that the UMAT material subroutine gives us $\Delta_{t}, \mathbf{q}_{n}, \mathbf{F}_{n}, \mathbf{F}_{n+1}$, being $\mathbf{q}$ the set of internal variables used in the model.

$$
\begin{gathered}
\mathbf{F}_{n+1}^{e}=\mathbf{F}_{n+1}\left(\mathbf{F}_{n+1}^{p}\right)^{-1} \\
\mathbf{F}_{n+1}^{p}=\exp \left(\sum_{\alpha} \Delta \gamma^{\alpha}\left(\mathbf{s}^{\alpha} \otimes \mathbf{m}^{\alpha}\right)\right) \mathbf{F}_{n}^{p} \\
\mathbf{S}_{n+1}=\mathbb{C}:\left(\frac{1}{2}\left(\left(\mathbf{F}_{n+1}^{e}\right)^{T} \mathbf{F}_{n+1}^{e}\right)-\mathbf{I}\right) \\
\tau_{n+1}^{\alpha}=\mathbf{S}_{n+1}:\left(\mathbf{s}^{\alpha} \otimes \mathbf{m}^{\alpha}\right)
\end{gathered}
$$




$$
\begin{gathered}
\Delta \gamma^{\alpha}=\Delta t \dot{\gamma}^{\alpha}\left(\tau_{n+1}^{\alpha}, g_{n+1}^{\alpha}\right) \\
g_{n+1}^{\alpha}=g_{n}^{\alpha}+\sum_{\beta} \mathrm{Q}^{\alpha \beta} h\left(\Gamma_{n+1}\right) \Delta \gamma^{\beta} \\
\Gamma_{n+1}=\Gamma_{n}+\sum_{\alpha} \Delta \gamma^{\alpha}
\end{gathered}
$$

The above set of equations was solved by using an elastic-predictor/plasticcorrector algorithm. The algorithm assumes a pure elastic trial step from time $t_{n}$ to $t_{n+1}$. Therefore, the variables in the first trial step $\left(^{*}\right)$ are updated as:

$$
\begin{gathered}
\mathbf{F}_{n+1}^{e *}=\mathbf{F}_{n+1}\left(\mathbf{F}_{n}\right)^{-1} \mathbf{F}_{n}^{e} ; \Delta \gamma^{\alpha *}=0 \\
g_{n+1}^{\alpha *}=g_{n}^{\alpha} ; \tau_{n+1}^{\alpha *}=\mathbf{S}_{n+1}^{*}:\left(\mathbf{s}^{\alpha} \otimes \mathbf{m}^{\alpha}\right)
\end{gathered}
$$

and the consistency of the assumption is checked, using equation 4.14, with:

$$
\mathbf{R}^{\alpha}\left(\Delta \gamma^{\alpha}\right)=\Delta \gamma^{\alpha *}-\Delta t \dot{\gamma}^{\alpha}\left(\tau_{n+1}^{\alpha *}, g_{n+1}^{\alpha *}\right)=0
$$

where $\mathbf{R}^{\alpha}\left(\Delta \gamma^{\alpha}\right)$ stands for the residual of the non-linear-equations set. If the above requirement is not fulfilled, the plastic slip increment $\Delta \gamma^{\alpha *}$ is updated by a Newton-Raphson scheme until the norm of the residual is lower than a tolerance. Note that $\tau_{n+1}^{\alpha *}, g_{n+1}^{\alpha *}$ must be updated every Newton-Raphson iteration.

\section{$4.2 \quad$ Finite-element modelling}

The response of the DS alloy was obtained by considering the representation of grains and crystal orientations explicitly. Since the specimens used in the study were oligocrystals, the whole gauge length of the samples were simulated instead of using a representative volume element (RVE). Figure 4.1 depicts some examples of the virtual specimens used in the present work. 


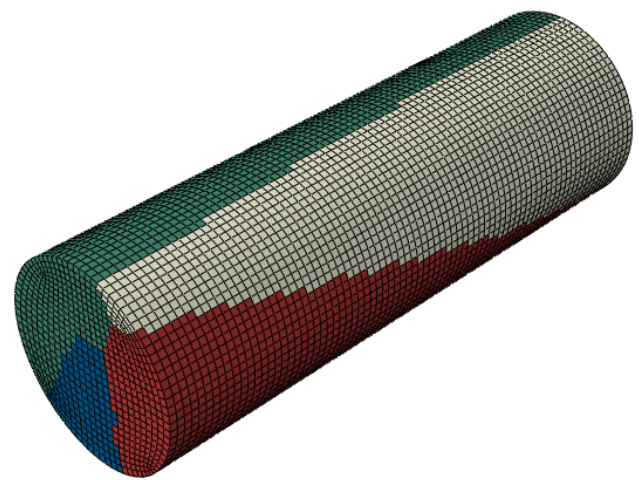

(a)

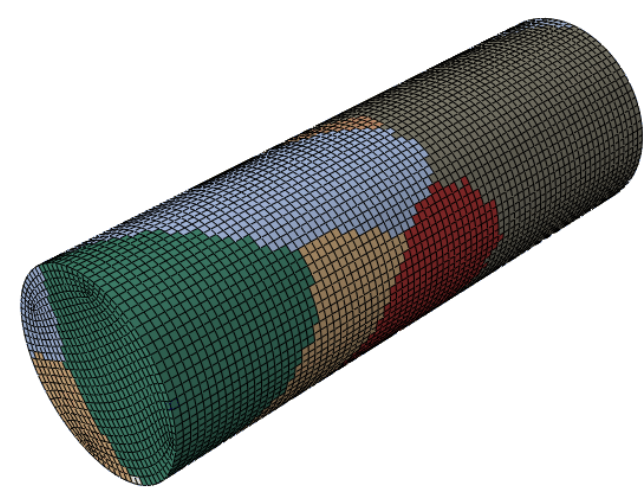

(c)

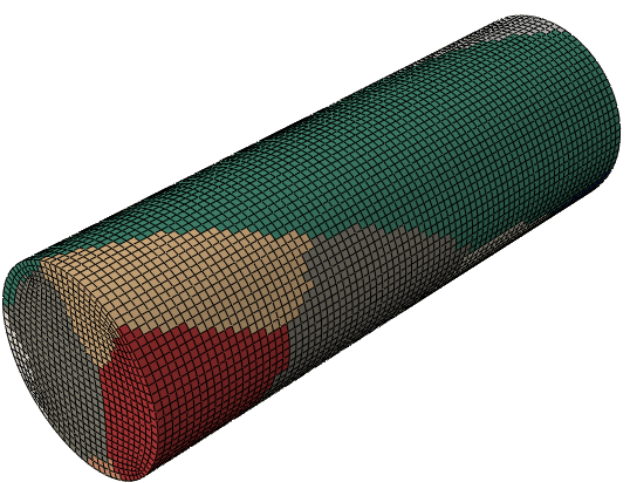

(b)

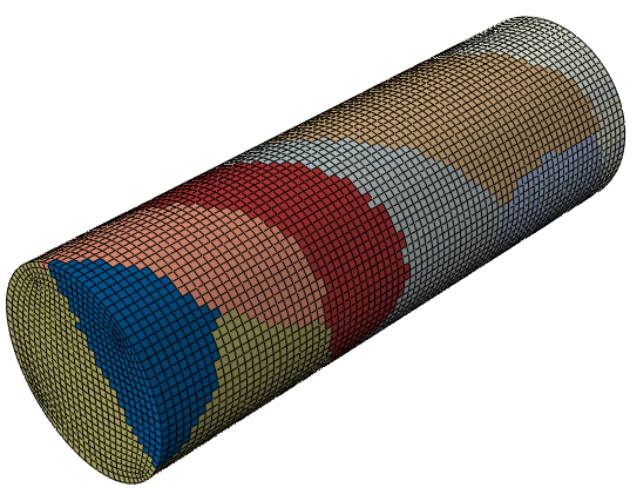

(d)

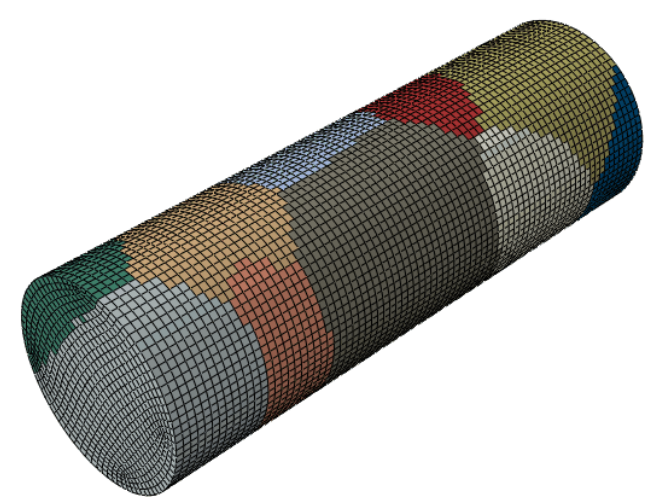

(e)

Figure 4.1: Illustration of the cylindrical FE models used to simulate the gauge length of the specimens. Each color represent a different grain and each sub-figure corresponds to a different sample orientation with respect to the grain-growth direction (a) $0^{\circ}$, (b) $30^{\circ}$, (c) $45^{\circ}$, (d) $60^{\circ}$, (e) $90^{\circ}$. Rotation angles with respect to the $y$-axis. 
The FE specimens were generated from a virtual cubic volume, with dimensions of $24 \times 24 \times 24 \mathrm{~mm}$, containing microstructural parameters (grain-size distribution, grain-shape and texture (crystallographic orientations)) that are representative of the studied material (Figure 4.2). That synthetic microstructure was created by using the Dream3D software [98], a microstructure builder. The shape and size characteristics of the grains were defined according to the information obtained from optical microscopy of the polished sections, which were already presented in Section 3.4 (Figure 3.18). To imitate the columnar structure, the shape of the grains was described as ellipsoids with an aspect ratio of 18:1:1 in the grain-growth direction ( $z$-axis). The size of the grains was characterised as the log-normal distribution of the equivalent-sphere diameter $(\mathrm{ESD})^{1}$ of each grain. A value of $9.2 \mathrm{~mm}$ for the mean equivalent-sphere diameter and $1.04 \mathrm{~mm}$ for the standard deviation were accurate enough to get a grain-size distribution similar to the experimental one, as can be seen in Figure $4.2 \mathrm{~b}$. It is worth mentioning that such values depend on the size of the virtual cubic volume [99] and were optimised for a volume of $24 \times 24 \times 24 \mathrm{~mm}^{3}$. The procedure to obtain the grain-size distribution of the synthetic microstructure was the same as that used in the experimental campaign ( Section 3.4).

As mentioned before, the FE specimens were generated from the synthetic volume. For that purpose, the Abaqus Scripting Interface (ASI) was employed [66]. A home-made script, written in Python programming language [100], begins by creating the geometry and mesh of the sample. Next, the grain ID is assigned to each element, defining sets of elements, by comparing the coordinates of each mesh-element centroid with the grid positions of the synthetic volume (Dream3D software returns a.$d x$ file with information about the position of each grain ID in the synthetic volume). Finally, the program defines the orientation of each grain through creating a material card and including the description of the [100] and [010] crystal axes with respect to the reference system $\left(\mathbf{e}_{x}, \mathbf{e}_{y}, \mathbf{e}_{z}\right)$ (Figure 4.3). The coordinates of those two vectors in the reference system are, respectively, the first and second row of the transformation

\footnotetext{
${ }^{1}$ Diameter of a sphere with the same volume as the grain
} 


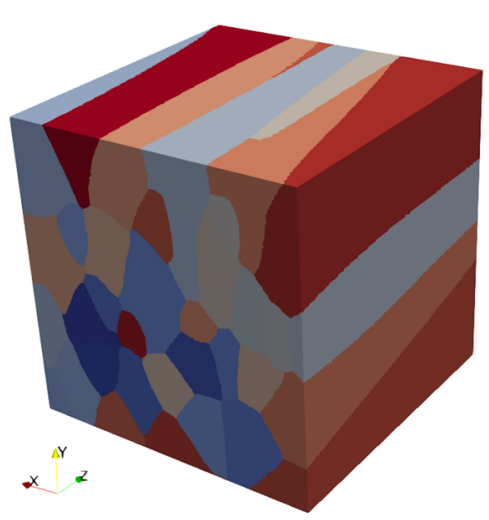

(a)

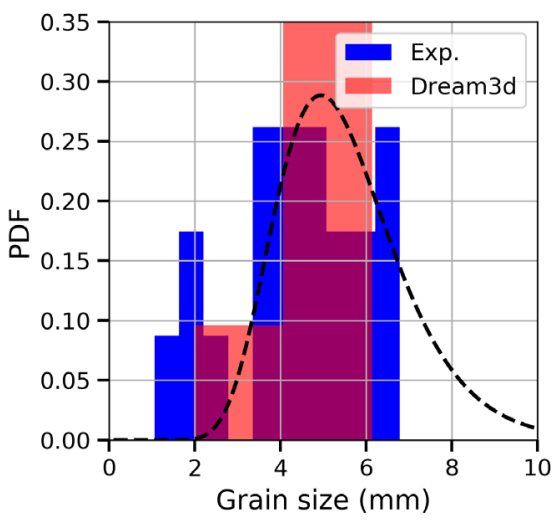

(b)

Figure 4.2: (a) Synthetic cubic volume and (b) comparison between the experimental and synthetic grain-size distribution. The dashed line represents the fitting of the experimental log-normal distribution (Section 3.4).

matrix $\mathbf{Q}^{\theta}\left(\phi_{1}, \Phi, \phi_{2}\right)$ based on the three Euler angles ${ }^{2}\left(\phi_{1}, \Phi, \phi_{2}\right.$ with Bunge convention. See Appendix B). In the equation 4.19, the $c$ stands for cos, $s$ for sin and the indexes 1, 2, 3 for the three Euler angles $\phi_{1}, \Phi, \phi_{2}$, in that order.

$$
\mathbf{Q}^{\theta}\left(\phi_{1}, \Phi, \phi_{2}\right)=\left(\begin{array}{ccc}
c 1 c 3-c 2 s 1 s 3 & c 3 s 1+c 2 c 1 s 3 & s 3 s 2 \\
-c 1 s 3-c 3 c 2 s 1 & c 1 c 2 c 3-s 1 s 3 & c 3 s 2 \\
s 2 s 1 & -c 1 s 2 & c 2
\end{array}\right)
$$

In the present work, two different types of orientations were used: DS-type and randomly distributed grain orientations. Randomly distributed orientation was required to replicate tests on polycrystal MAR-M247 from literature and validate the predictive capability of the model. On the one hand, DS-type orientation was generated assuming a fibre texture in which all grains have a $<100>$ direction oriented near the $z$-axis, while the perpendicular directions are quasi-randomly oriented. The $z$-axis was chosen as grain-growth direction by convention. This kind of crystallographic orientation corresponds to the common texture obtained in DS superalloys [101, 102, 20]. The normal distri-

\footnotetext{
${ }^{2}$ Euler angles are often used in materials science to describe the crystal orientations.
} 


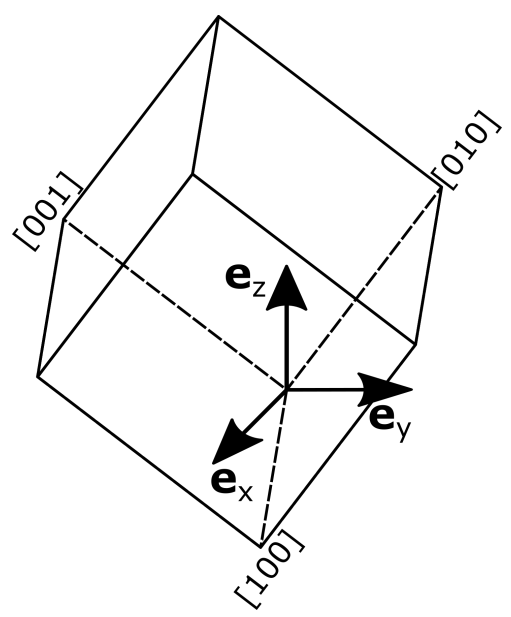

Figure 4.3: Crystal orientation respect to the reference system

butions of the Euler angles used to generate the fibre texture in the present work were defined by $\phi_{1}=20^{\circ} \pm 90^{\circ}, \Phi=0^{\circ} \pm 4^{\circ}, \phi_{2}=32^{\circ} \pm 20^{\circ}$. The values were not measured experimentally but obtained from the ones reported by R.J. Kashinga et al. [20] for another DS Ni-base superalloy. The reasons of this decision were twofold: due to the difficulty of measuring large areas through electron backscatter diffraction (EBSD) and due to the fact that our goal is to generate a virtual specimen equivalent to that used in experiments but not the same.

On the other hand, randomly distributed orientations were specified according to the instructions given in [103] to generate random aggregates of cubic crystals. It is worth mentioning that randomly distributed orientation cannot be generated from random uniform distributions of the three Euler angles because the Euler space is not metric. The procedure is based on choosing two random vectors $\mathbf{u}, \mathbf{v}^{\prime}$ and calculate the vector $\mathbf{v}$ that is contained in the plane $\overline{\mathbf{u v}^{\prime}}$ and is perpendicular to $\mathbf{u}$. Then, the orthogonal vector $\mathbf{w}=\mathbf{u} \times \mathbf{v}$ is computed. Since the columns of a rotation matrix are nothing but the components of three orthogonal unit vectors, $\mathbf{u}, \mathbf{v}$ and $\mathbf{w}$ are used to define the matrix that relates the reference and crystal axes.

Finally, Figure 4.4 shows a comparison between the texture generated for DS virtual samples and that for virtual samples with random orientation. It 
can be clearly seen the fibre texture in $z$-direction for the DS case. The quality of the algorithm for generating random orientations was also checked (4.4c).

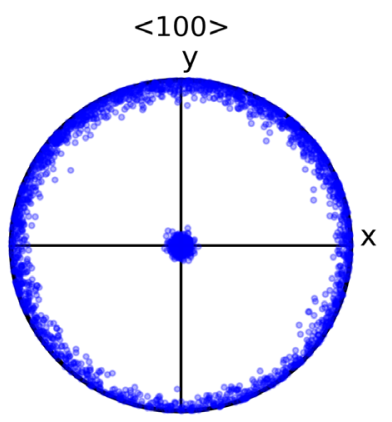

(a)

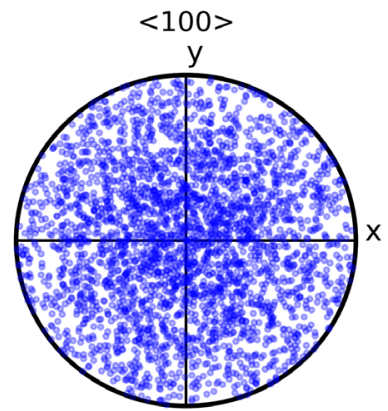

(b)

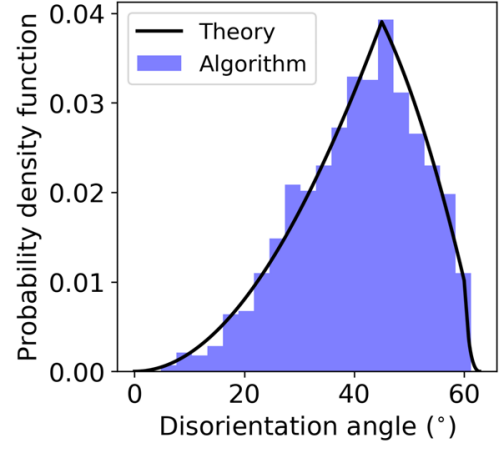

(c)

Figure 4.4: Stereographic projection of the crystallographic orientation for the (a) DS texture and (b) random orientation. 1000 grains were employed for the realizations. Plot (c) compares the PDF of the disorientation angle from the random realization to the theoretical value of perfect randomness [103].

\subsection{Parameter identification}

Once the crystal structure is generated and the texture is defined, the next step prior to running the simulation consists in determining the constants of the model. In the present case, this task was divided into obtaining the elastic stiffness tensor $\mathbb{C}$, the coefficients of the strength-interaction matrix $\mathbf{Q}^{\alpha \beta}$, the hardening-law constants $h_{0}, \tau_{s}, \tau_{0}$ and the parameters $\dot{\gamma}_{0}, m$ that define the rate-sensitivity of the viscoplastic law.

Due to the cubic crystal system, the elastic stiffness tensor is described by the three independent elastic constants $C_{11}=258.6 \mathrm{GPa}, C_{12}=167.0 \mathrm{GPa}$ and $C_{44}=125.0 \mathrm{GPa}$. The values were obtained from [104], where the elastic properties of the single-crystalline alloy MAR-M200 were experimentally measured. The reason of using such constants grounds on the fact that MAR-M247 is a modification of MAR-M200 with very similar chemical composition. As will be shown later, the decision led to accurate results. 
In relation to the plastic behaviour, the dimensionless coefficients $q_{1}=1.00$, $q_{2}=1.00, q_{3}=5.38, q_{4}=0.68, q_{5}=1.12$ and $q_{6}=0.96$ of the strengthinteraction matrix were adapted from the values presented in [105] for FCC crystals. In that work, the evolution of the critical resolved-shear stress was formulated according to a dislocation-based model. Consequently, the coefficients of the dislocation interaction matrix $a^{\alpha \beta}$ from [105] needed to be adapted to the present phenomenological model. To that end, equation 4.20 was defined.

$$
\mathbf{Q}^{\alpha \beta}=\frac{a^{\alpha \beta}\left(\sum_{i=1}^{12} a^{\alpha i}\right)^{-0.5}}{a^{11}\left(\sum_{i=1}^{12} a^{1 i}\right)^{-0.5}}
$$

The set of parameters $h_{0}, \tau_{s}, \tau_{0}$, which describes the hardening behaviour, were fitted by inverse analysis, i.e. from experimental curves. Since only one deformation mode is assumed for the studied alloy (octahedral slip), just information from one of the two tested directions was needed to fit the constants. For that purpose, tests performed under quasi-static conditions in grain-growth direction were chosen because grain orientations are more uniform with respect to the loading axis. Moreover, good initial guess values can be defined from the simple case in which the crystal is stretched in one of the $<100>$ directions. The initial guess values $h_{0}^{0}=2.82 \tau_{0}, \tau_{s}^{0}=2.26 \tau_{0}$ and $\tau_{0}^{0}=0.97 \tau_{0}$ were considered assuming that all plastic deformation is accommodated by one slip system. $h_{0}=1.36 \tau_{0}, \tau_{s}=2.26 \tau_{0}$ and $\tau_{0}=\tau_{0}$ were the values obtained after the inverse analysis of $0^{\circ}$ tests using a virtual sample with 6800 elements (element size of $0.3 \times 0.3 \times 0.3 \mathrm{~m}^{3}$ approximately). The reduced number of elements did not have an impact on the accuracy of the macroscopic response (see Appendix $\mathrm{C}$ ) while decreased the computational cost to 1.5 hours $(6 \mathrm{x}$ Intel (R) Xeon (R) CPU E5-2640 v4@ $2.40 \mathrm{GHz}$ ) and made easier the optimization procedure. It is important to note that hardening constants are expressed as a function of $\tau_{0}$, which value has been considered as confidential data by ITP Aero company.

Finally, the yield strength evolution in $0^{\circ}$ direction at the different strain rates allowed the determination of parameter $m=0.0015$, being the reference slip rate $\dot{\gamma}_{0}$ equal to $0.001 \mathrm{~s}^{-1}$. The low value of $m=0.0015$ indicates low strain- 
rate sensitivity. For the shake of clarity, all values of the model are listed in Table 4.3.

Table 4.3: Parameters of the CP model for MAR-M247

\begin{tabular}{ccccccc}
\hline$C_{11}$ & $C_{12}$ & $C_{44}$ & $h_{0}$ & $\tau_{0}$ & $\tau_{s}$ & $m$ \\
\hline $258.6 \mathrm{GPa}$ & $167.0 \mathrm{GPa}$ & $125.0 \mathrm{GPa}$ & $1.36 \tau_{0}$ & $\tau_{0}$ & $2.26 \tau_{0}$ & 0.0015 \\
\hline$\dot{\gamma}_{0}$ & $q_{1}$ & $q_{2}$ & $q_{3}$ & $q_{4}$ & $q_{5}$ & $q_{6}$ \\
\hline $0.001 \mathrm{~s}^{-1}$ & 1.00 & 1.00 & 5.38 & 0.68 & 1.12 & 0.96 \\
\hline
\end{tabular}

\subsection{Results}

After identifying all constants of the model, the room-temperature experimental tests performed along $0^{\circ}$ and $90^{\circ}$ direction were simulated at different strain rates $\left(2.5 \cdot 10^{-4} \mathrm{~s}^{-1}, 150 \mathrm{~s}^{-1}\right.$ and $\left.500 \mathrm{~s}^{-1}\right)$. It should be recalled that experiments along $90^{\circ}$ direction were not used during the optimization procedure of the constants and the presented results are predictions of the CPFEM strategy.

The specimens were discretised with 0.15-mm-size eight-node fully-integrated hexahedral elements (C3D8). The 3mm-diameter specimens, those used for tests at $150 \mathrm{~s}^{-1}$, were replicated with 41400-element mesh and the $4 \mathrm{~mm}$ diameter specimens with 55040-element mesh (see Figures 4.5 and 4.6). The prescribed displacement was the same, equal to $1.5 \mathrm{~mm}$, in all tests and the total time of the loading step was adjusted to get the three different strain rates. The quasi-static problem was solved with the Abaqus static solver while the dynamic implicit version was used for the high-strain-rate virtual tests. In this latter case, two time increments were set to achieve stress-wave propagation and time-cost-effective simulations alike. Moreover, the convergence of the integration scheme in the elastic-plastic transition is enhanced. During the elastic regime, a time increment 10 times larger than the Courant number was chosen, whereas a time increment 100 times larger was use used for the inelastic regime once the stress equilibrium on the specimen was reached (beginning of plastic deformation). More information in Appendix D. 


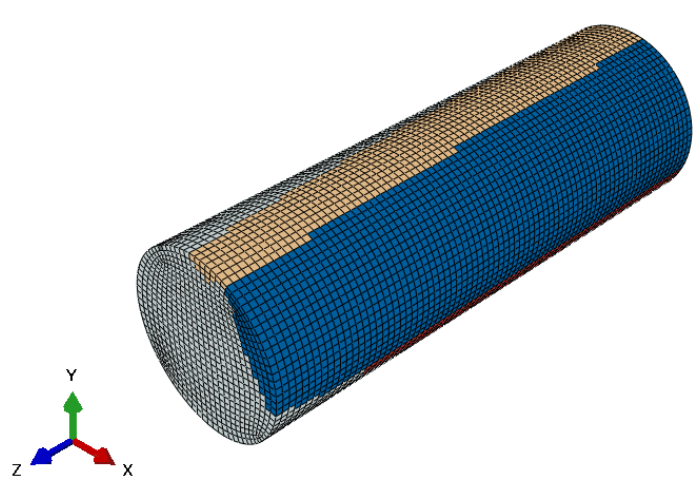

(a)

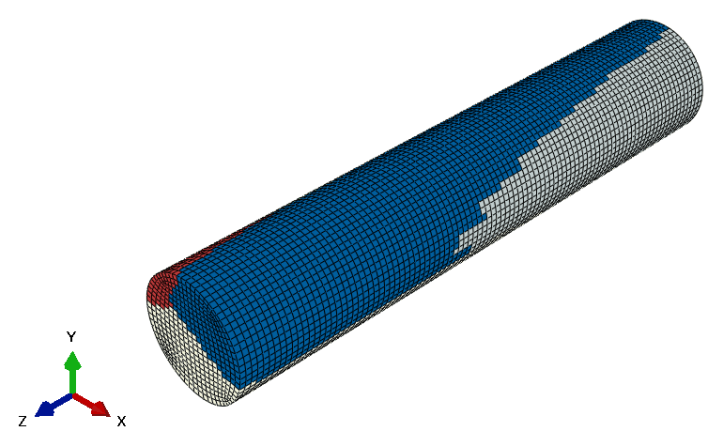

(c)

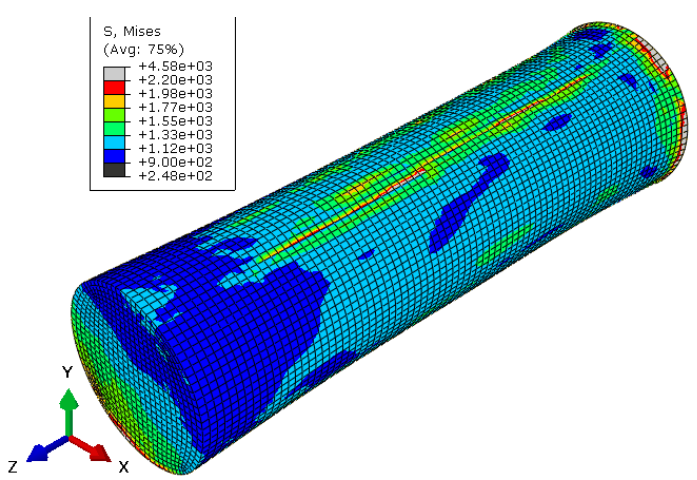

(b)

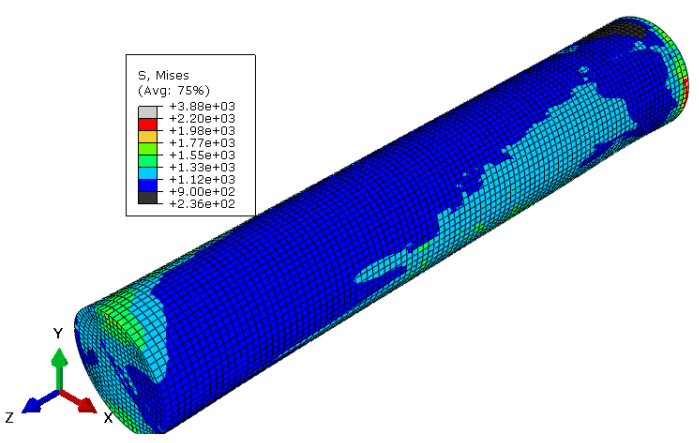

(d)

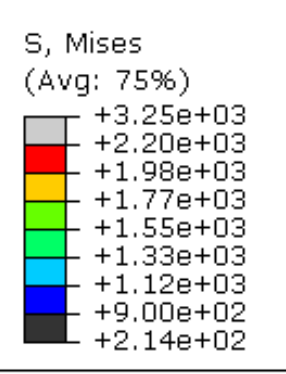

(e)

Figure 4.5: Some virtual $0^{\circ}$-oriented samples used in the simulations in the undeformed state $(\mathrm{a}, \mathrm{c})$ and after deformation $(\mathrm{b}, \mathrm{d})$. The contour plots show the stress concentration at grain boundaries. Images (a) and (b) are samples with $4 \mathrm{~mm}$ diameter while (c) and (d) are samples with $3 \mathrm{~mm}$ diameter. 


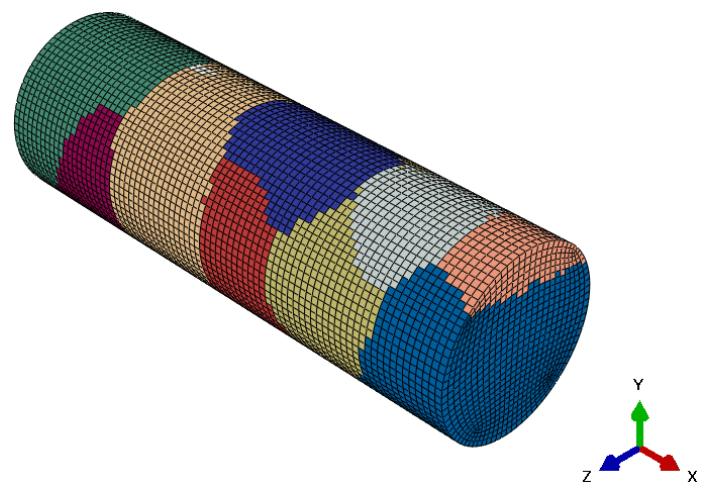

(a)

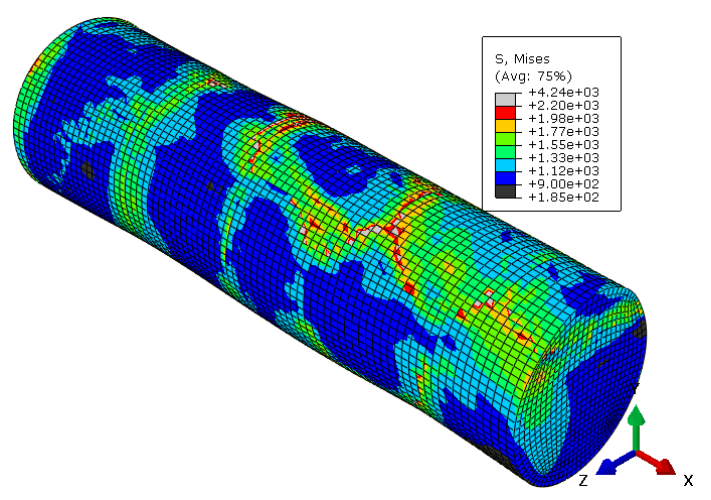

(b)

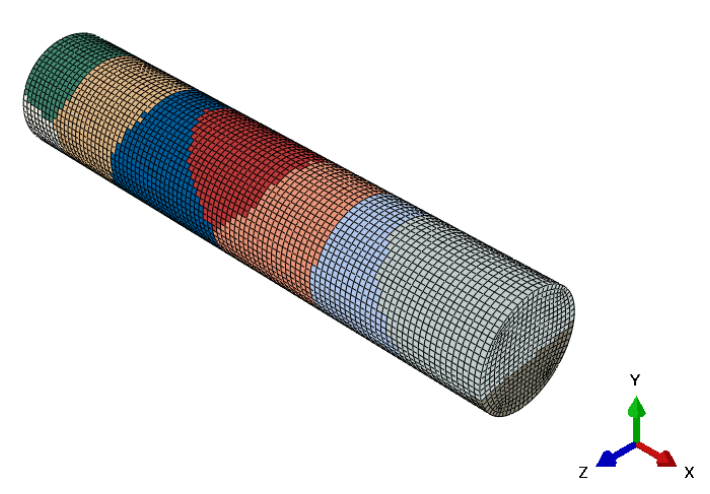

(c)

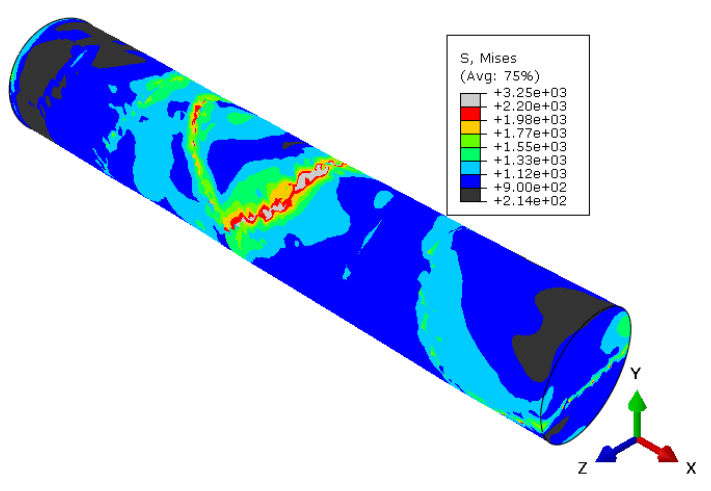

(d)

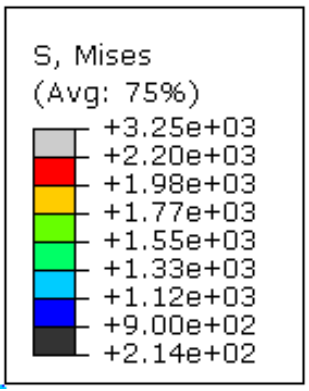

(e)

Figure 4.6: Some virtual $90^{\circ}$-oriented samples used in the simulations in the undeformed state $(\mathrm{a}, \mathrm{c})$ and after deformation $(\mathrm{b}, \mathrm{d})$. The contour plots show the stress concentration at grain boundaries. Images (a) and (b) are samples with $4 \mathrm{~mm}$ diameter while (c) and (d) are samples with $3 \mathrm{~mm}$ diameter. 
The experimental true stress-strain curves of MAR-M247 DS alloy are plotted in Figure 4.7 along with the results from simulations. Although the experiments were already presented in Section 3.4 to analyse the strain-rate sensitivity, a thorough analysis was done in this section for completeness. In each graph, there are two families of tests that belong to the specimens obtained parallel $\left(0^{\circ}\right)$ and perpendicularly $\left(90^{\circ}\right)$ to grain-growth direction. It can be seen that the Young's modulus, yield strength and hardening behaviour depend on sample orientation in the same way for all strain rates. Regarding the elastic behaviour, the Young's modulus varies by $33 \%$ depending whether the samples are parallel (mean value of $36.72 \tau_{0} / \Delta \varepsilon_{\max }$ ) or perpendicular (mean value of $\left.48.57 \tau_{0} / \Delta \varepsilon_{\max }\right)$ to the solidification direction. The yield strength, calculated as the 0.2 offset yield point, also changes. The larger values correspond to that in the $0^{\circ}$ orientation $\sigma_{0.2}=2.37 \tau_{0}$, while the performance in the transverse direction goes down to quantities close to $2.11 \tau_{0}$. Moreover, a slight variation on hardening is observed when comparing the two orientations. It is worth noting that the experiments report some dispersion since oligocrystals were tested. These differences among curves are more noticeable in the case of $90^{\circ}$ specimens due to the random orientation of the crystals.

The simulation strategy presented previously was able to faithfully predict the response of the alloy in $90^{\circ}$ orientation (Figure 4.7). Two independent virtual samples were employed per orientation and strain-rate condition. The mean value with the standard deviation were plotted using markers. The $<100>$-fibretexture assumption and the particular crystal-orientations adopted for virtualsample generation were confirmed as valid according to the equivalence between experimental and numerical results along both orientations in the elastic regime (Figure 4.8). Furthermore, the suitability of the elastic stiffness tensor is also demonstrated. The experimental difference in the yield strength between the two sample configurations is also captured by simulations. It is connected to the different maximum Schmid factors. The higher the Schmid factor, the lower the external-applied stress required to move dislocations. At $0^{\circ}$, the texture is highly oriented in $<001>$ direction and the maximum Schmid factor for most of crystals is close to 0.408 . Conversely, the grain orientations of $90^{\circ}$ specimens are more random and the maximum Schmid factor may take values between 


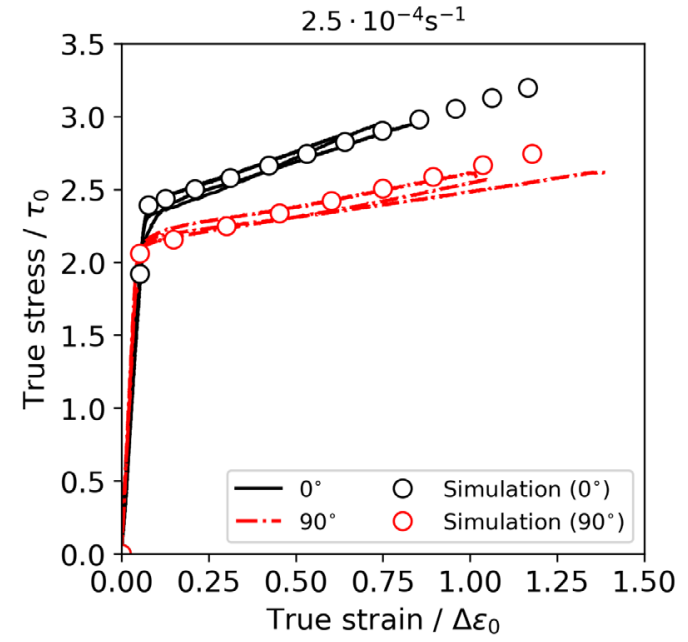

(a)

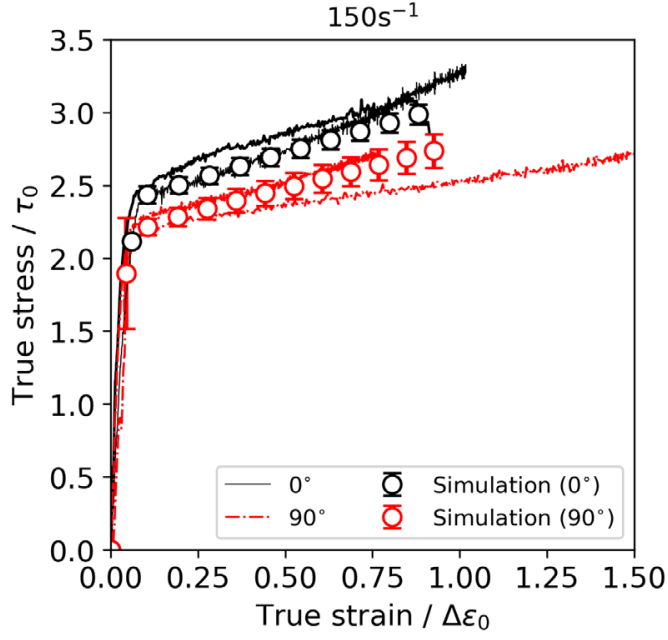

(b)

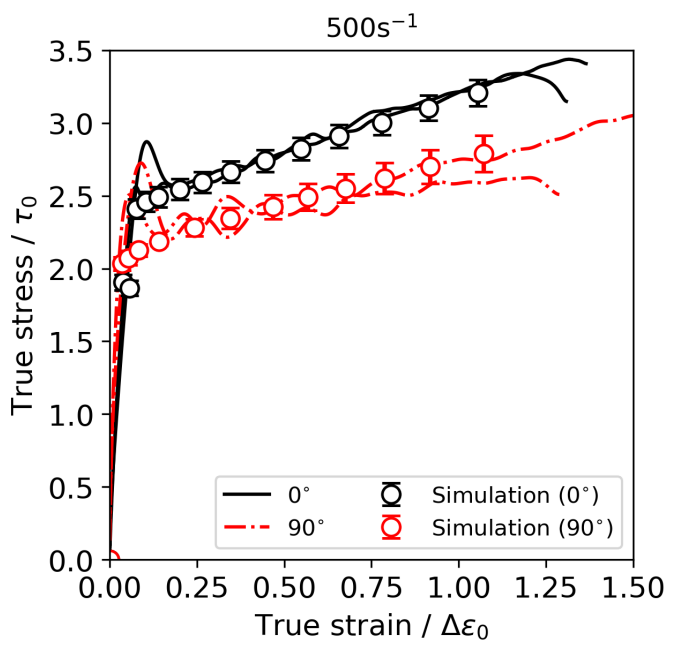

(c)

Figure 4.7: Experimental (lines) and numerical (circles) true stress-strain curves for MAR-M247 DS alloy at room temperature under (a) quasi-static and $(b, c)$ dynamic regimes and different loading directions. 


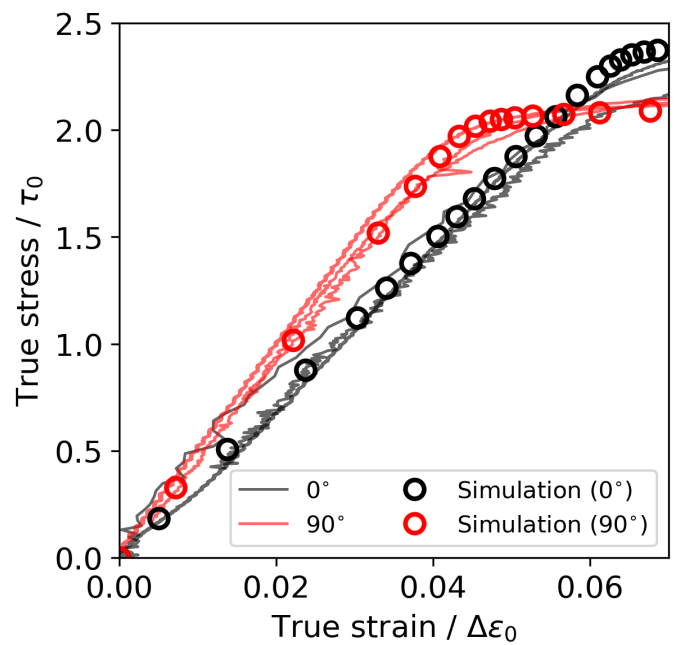

Figure 4.8: Elastic part of the experimental (lines) and numerical (circles) true stress-strain curves for the quasi-static tests.

0.408 and 0.50 . For example, in the $90^{\circ}$ simulation, on average, $21 \%$ of grains had maximum Schmid factors below 0.44 and $40 \%$ above 0.47. Furthermore, the grain structure resembles the isostress condition of heterogeneous materials. As a consequence, the grains with higher Schmid factor (softer grains) tend to accommodate more plastic deformation at the beginning of yielding, as can be seen in Figure 4.9. However, since the CRSS depends implicitly on the total amount of plastic strain, grains with higher plastic strain harden faster. Therefore, there is a moment at which the grains with lower Schmid factor begin to provide less resistance to dislocation slip. Figure 4.9 shows how the probability density function of plastic deformation rises for grains with low Schmid factor and drops for the ones with high Schmid factor when the accumulated plastic strain increases.

The stress concentration at grain boundaries was also analysed (see Figures 4.5 and 4.6). To that end, the last frame of all simulations was chosen. The maximum macroscopic or global von-Mises stress $\bar{\sigma}$ (externally measured) experienced by each sample was set as threshold and the amount of elements with larger von Mises stress $\bar{\sigma}_{\mu}$ (local field) was computed. Figure 4.10 collect these results. 


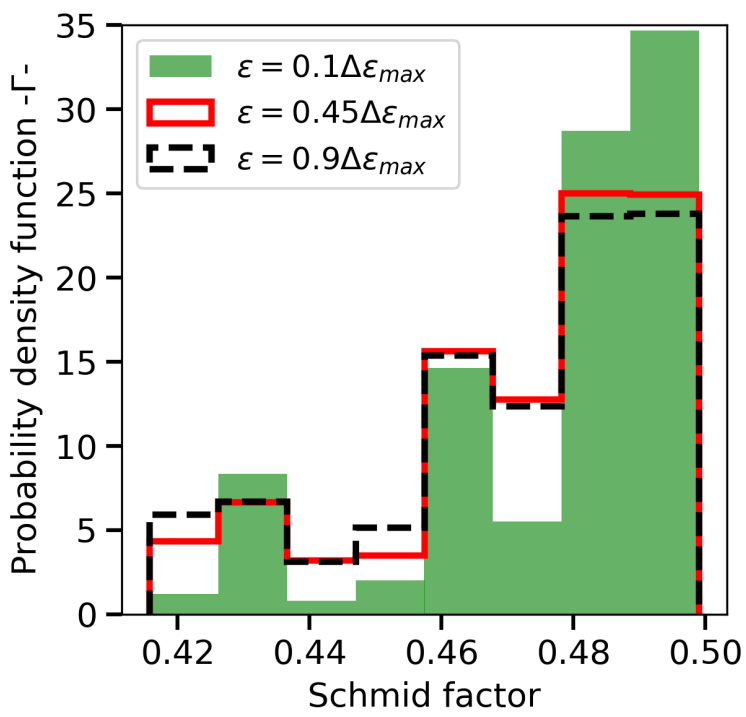

Figure 4.9: Amount of plastic slip $\Gamma$ accommodated by grains with different maximum Schmid factor for $90^{\circ}$ samples at the beginning of plastic deformation $\left(0.1 \Delta \varepsilon_{\max }\right)$, at the middle of the test $\left(0.45 \Delta \varepsilon_{\max }\right)$ and at the final stage $\left(0.9 \Delta \varepsilon_{\max }\right)$.

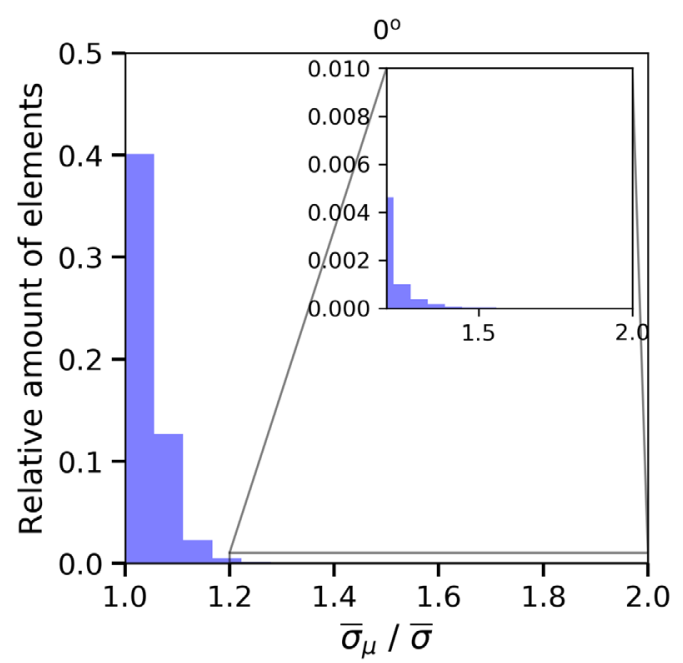

(a)

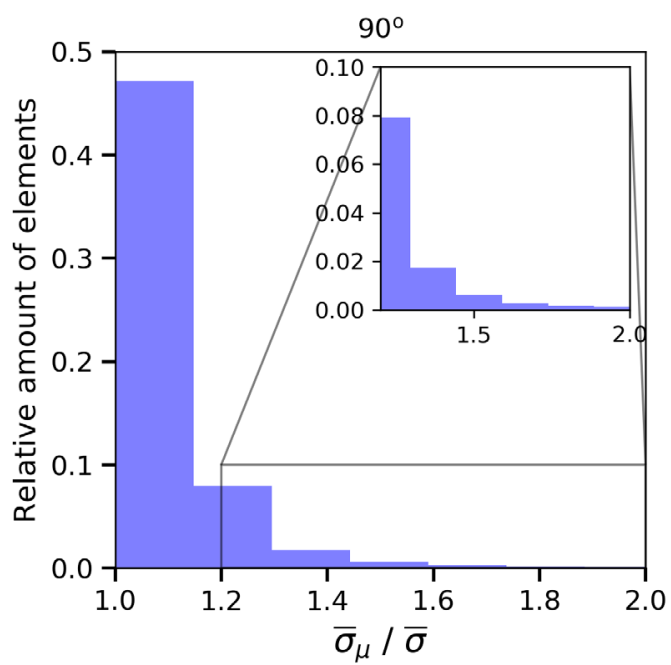

(b)

Figure 4.10: Amount of elements with local stress (von-Mises stress) values $\bar{\sigma}_{\mu}$ larger than the global value supported by the sample $\bar{\sigma}$ for (a) $0^{\circ}$ - and (b) $90^{\circ}$-oriented samples. The data involve the last frame of all simulations. 
The specimens oriented perpendicularly to grain-growth direction have, approximately, ten percent of elements with a local von-Mises stress 1.2 times larger than the macroscopic value and exceptionally, there are elements reaching concentration factors $\bar{\sigma}_{\mu} / \bar{\sigma}$ close to 2 . However, these stress ratios for the $0^{\circ}$-oriented samples are in the range of 1.0-1.2. Their highly oriented texture means that the mechanical properties among grains are quite similar and the stress concentration close to the crystal-crystal transition is rather small, unlike $90^{\circ}$ specimens, which also present grain boundaries perpendicular to loading direction. So, it seems clear that $90^{\circ}$ specimens are more likely to nucleate cavities, as expected [94]. Figure 4.11 shows an example of cracks on the surface of a tested sample that are probably originated due to stress concentrations on grain boundaries, inter-dendritic regions and/or zones with high density of precipitates. These cracks were observed on both $0^{\circ}$ - and $90^{\circ}$ - oriented samples.

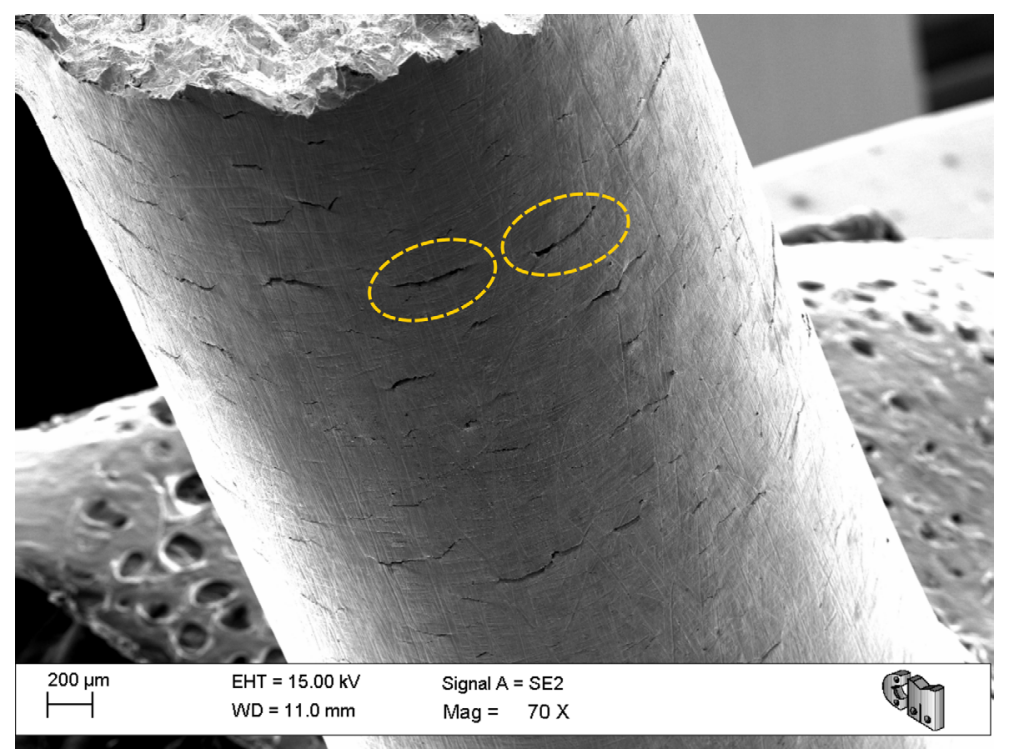

Figure 4.11: Detailed view of the surface of a tested MAR-M247-DS sample.

Besides experiments along $90^{\circ}$ direction, data from literature was employed to verify the prediction capability of the numerical model. In practice, the $0.2 \%$ yield strength and elastic constants, such as the Young's modulus, shear modulus and Poisson's ratio of quasi-polycrystal [92] and polycrystal [106] MARM247 alloys were compared with results from simulations. 
M. Kaufman [92] tested MAR-M247 specimens cut from blisks to provide information about tensile, creep-rupture, fatigue and crack-growth rate properties. However, in this work, $0.2 \%$ yield strength values were only of interest. The smooth tensile specimens had a diameter of $12.7 \mathrm{~mm}$ and a length of 25.4 $\mathrm{mm}$ in the gauge section. With respect to the microstructure, relatively uniform equiaxed grains with sizes up to $3.2 \mathrm{~mm}$ were reported. The virtual sample (Figure 4.12) was generated by using the Dream.3D software in a similar way to that employed for the directionally solidified specimens. The grain-size distribution was not available in the report, so a mean grain size of $d_{g}=2.72 \mathrm{~mm}$ and a standard deviation of $d_{S D}=0.21 \mathrm{~mm}$ were used. This distribution gave uniform grains and some probability (11\% approximately) to get $3.2 \mathrm{~mm}$-sized grains. The finite-element sample had the same dimensions as the gauge length of the real specimens and was discretised with 43232 C3D8 hexahedral elements of $0.45 \times 0.45 \times 0.45 \mathrm{~mm}^{3}$ volume approximately, getting 255 different grains with randomly distributed orientation. Three virtual tests were performed keeping the same sample structure but with different grain orientations. The sample was loaded by applying a prescribed displacement, at a rate of $6.5 \cdot 10^{-3} \mathrm{~mm} / \mathrm{s}$, to one end while constraining the opposite end in the displacement direction $(z-$ direction). Non-desired translations and rotations were avoided by restricting the movement of two nodes of the base, in $x$ - and $y$ - direction for one node and in $x$ - or $y$-direction for the other one. The mean $0.2 \%$-yield-strength value from simulations was $858 \pm 7.2 \mathrm{MPa}$, which is comparable to the $854 \pm 19$ value $\mathrm{MPa}$ given by Kaufman.

Secondly, J-H. Liao et al. [106] investigated the tensile behaviour of MARM247 superalloy with different additions of rhenium. In that study, the samples had a gauge length of $26 \mathrm{~mm}$ and a diameter of $6.3 \mathrm{~mm}$. Unlike previous cases, MAR-M247 specimens had an average grain size of $90 \mu \mathrm{m}$, precluding the simulation of the whole gauge length microstructure due to the computational cost. Therefore, the tensile response of the polycrystal was obtained by computational homogenization of an RVE of the material microstructure (Section 2.2.3 explains the basis of polycrystal homogenization). In this approach, the RVE, with 469 grains, was discretised with $30 \times 30$ x 30 cubic finite elements and each grain was represented with several elements (Figure 4.13). 


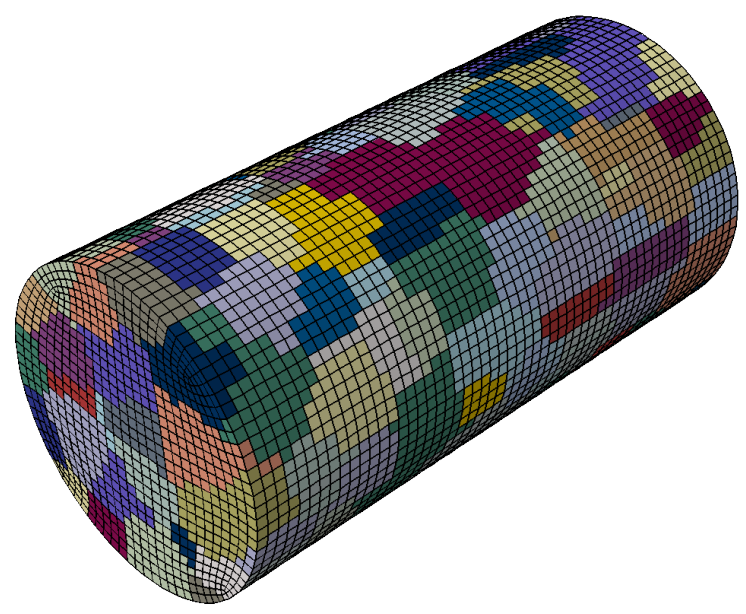

Figure 4.12: Virtual specimen for simulating Kaufman tensile tests with 255 different equiaxed grains.

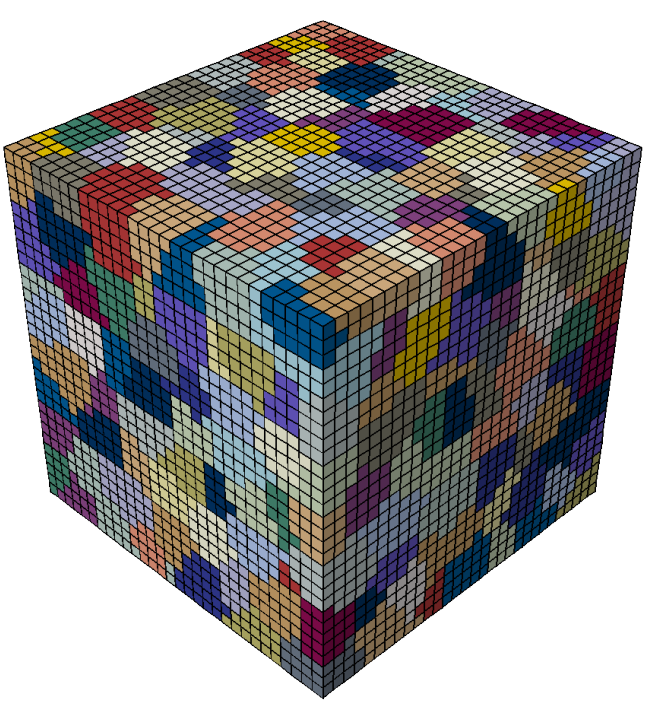

Figure 4.13: Realistic RVE of MAR-M247. 
Again, the microstructure was synthetically generated by using Dream.3D software. Equiaxed grains were assumed and the grain-size distribution was defined to obtain a mean grain size of $d_{g}=90 \mu \mathrm{m}$ and a standard deviation of $d_{S D}=0.20 \mu \mathrm{m}$. No information about the grain-size dispersion was found in the report, so quite uniform grain sizes were supposed. The grain orientation was defined as randomly distributed. One virtual test was only run since 469 different grains is an enough number of crystals to obtain an independent behaviour of the particular random-orientation realisation [39].

Periodic boundary conditions were applied on the RVE faces to get the homogenized polycrystal response [39]. The model was subjected to tensile deformation in the $z$-direction up to a value of $10 \%$ at a strain rate of $2.5 \cdot 10^{-4} \mathrm{~s}^{-1}$. In this way, the boundary conditions were defined as (Section 2.2.3):

$$
\begin{gathered}
\dot{u}_{z}\left(M_{z}\right)=\dot{e} L_{0} \\
P_{x}\left(M_{z}\right)=P_{y}\left(M_{z}\right)=0 \\
P_{i}\left(M_{x}\right)=P_{i}\left(M_{y}\right)=0, i=x, y, z
\end{gathered}
$$

where $M_{i}$ stands for the master node of each pair of opposite faces and $P_{i}$ for the nodal force in the specified direction of the corresponding master node $M_{i}$. The strain and stress values were obtained from the displacement $u_{z}$ and reaction forces $F_{z}$ on the RVE face $z=L_{0}$.

The comparison between experimental results and the numerical prediction are plotted in Figure 4.14 as engineering stress-strain curves. In this graph, there are two curves labelled as experimental results. The upper one (black continuous line) corresponds to the result presented by J-H. Liao et al. [106] and when comparing with the simulation, it seems that the prediction underestimates the response of the alloy. However, this difference is based on the fact that the constants of the model were fitted with samples characterised by a mean grain size of $5.5 \mathrm{~mm}$, whereas J-H. Liao et al. tested $90 \mu \mathrm{m}$ grain-size specimens. It is well established the hardening effect on the flow stress when the grain size is reduced due to the dislocation pile-ups at the grain boundaries and the generation of geometrically-necessary dislocations [105]. 


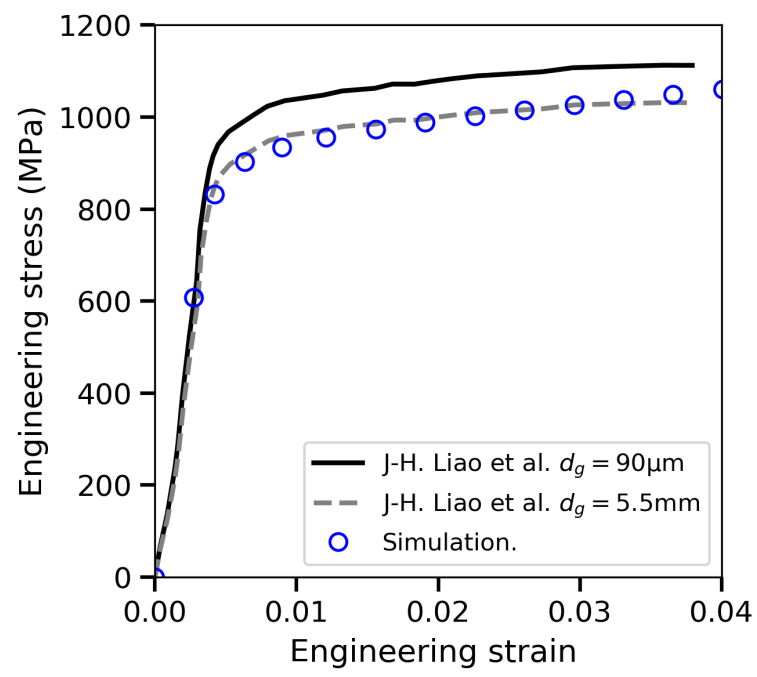

Figure 4.14: Experimental and numerical (prediction) engineering stressstrain curve for polycrystal MAR-M247. Experimental results from [106] were scaled down for $5.5 \mathrm{~mm}$ by using the Hall-Petch expression and data from [109].

E.O Hall [107] and N.J Petch [108] formulated the dependence of the yield stress $Y$ with the grains size $d_{g}$ according to the equation:

$$
Y=Y_{\infty}+C_{H P} d_{g}^{-0.5}
$$

where $Y_{\infty}$ is the yield stress of a polycrystal with very large grain sizes and $C_{H P}$ is a material constant.

With the aim of enabling a comparison between experimental and numerical results, the data reported for $d_{g}=90 \mu \mathrm{m}$ was extrapolated for $d_{g}=5.5 \mathrm{~mm}$ (grey dashed line) by using the Hall-Petch expression. The constants $Y_{\infty}=896 \mathrm{MPa}$ and $C_{H P}=24.7 \mathrm{MPa} \sqrt{\mathrm{mm}}$ were fitted from the yield strength dependence on the grain size reported in [109] for the alloy CM 247LC, a modified version of MAR-M247 with low carbon content (Table 4.4).

Finally, Table 4.5 summarises the comparison between the experimental and predicted $0.2 \%$-yield-strength values for quasi-polycrystal and polycrystal MAR-M247 specimens. Besides the plastic behaviour, the isotropic elastic constants were computed from the polycrystal simulations and compared with the values obtained from literature. In the light of the results, it can be concluded 
Table 4.4: Variation on the yield strength as a function of grain size

\begin{tabular}{cc}
\hline Yield strength $(\mathrm{MPa})$ & Grain size $(\mu \mathrm{m})$ \\
\hline 1010 & 85 \\
985 & $150-200$ \\
939 & $2000-3000$ \\
\hline
\end{tabular}

Table 4.5: Experimental 0.2\%-yield-strength values and numerical predictions for polycrystal and quasi-polycrystal MAR-M247 specimens. The predicted isotropic elastic constants are also compared with the values from literature [104].

\begin{tabular}{ccc}
\hline & Bibliography & CPFEM predictions \\
\hline Polycrystal structure & $\sigma_{0.2}=909 \mathrm{MPa}$ & $\sigma_{0.2}=905 \mathrm{MPa}$ \\
Quasi-polycrystal & $\sigma_{0.2}=854 \pm 19 \mathrm{MPa}$ & $\sigma_{0.2}=858 \pm 7.2 \mathrm{MPa}$ \\
Isotropic elastic consts. & $E=223.5 \mathrm{GPa}, \nu=0.29$ & $E=224 \mathrm{GPa}, \nu=0.32$ \\
\hline
\end{tabular}

that the model is able to predict results from tensile tests on specimens with quite different microstructure; and therefore, could be used as a tool for virtual testing. This strategy allows the researches to use the results from simulations to fit the yielding constants of macroscopic plasticity models [110], which are important when testing large structural components. As an example of virtual testing, Figure 4.15 and Figure 4.16 plot, respectively, the true stress-strain curves and the 0.2\%-yield-strength and Young's-modulus evolution for different orientations between the parallel $\left(0^{\circ}\right)$ and perpendicular $\left(90^{\circ}\right)$ direction to grain-growth direction (samples in Figure 4.1). Two virtual samples were simulated for each intermediate configuration. In all cases, the rotations were done around the $y$ - axis. The virtual results show that the lowest yield-strength values are obtained with the $90^{\circ}$ orientation, followed by the $30^{\circ}$-oriented samples. The simulations predict a similar yield-strength performance between $0^{\circ}$ - and $45^{\circ}$-oriented samples, although the strain-hardening rate is larger in the latter case. However, its Young's modulus is higher and therefore, a hypothetical $45^{\circ}$ configuration would present a worse thermal fatigue behaviour since the thermal strains are higher. 


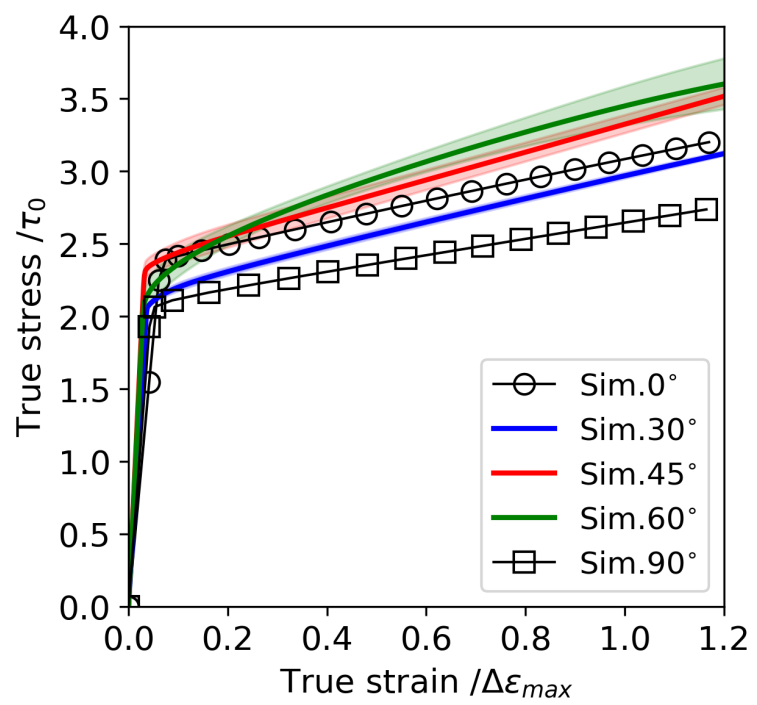

Figure 4.15: Quasi-static true stress-strain curves for MAR-M247 DS alloy tested virtually at different angles with respect to grain-growth direction. The microstructure of the samples is shown in Figure 4.1.

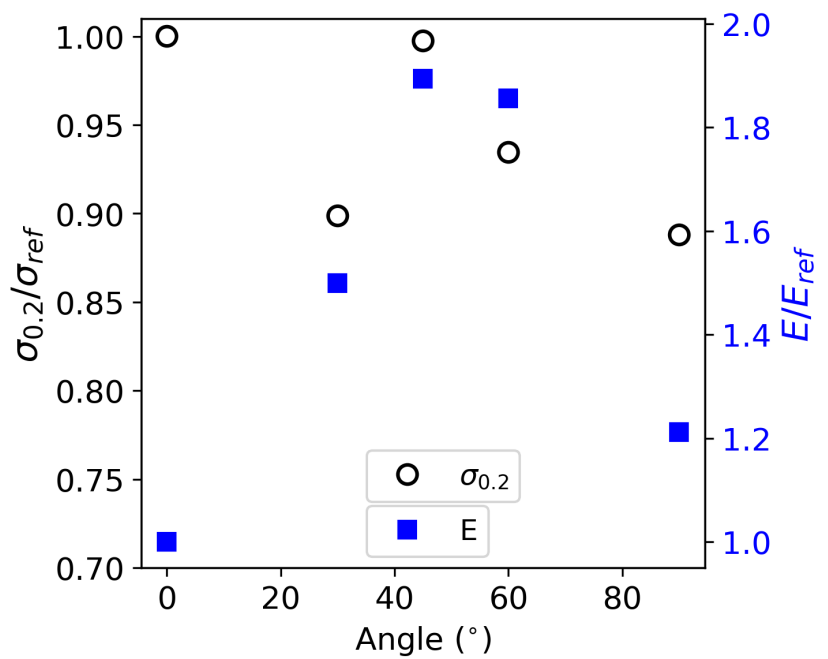

Figure 4.16: Prediction of the evolution of the normalised yield strength and Young's modulus with respect to loading direction. 


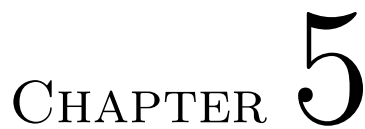

\section{Conclusions and Future Work}

\subsection{Conclusions}

The next conclusions synthesises the work presented throughout this thesis.

- The shortcoming of the original Johnson-Cook constitutive model to deal with alloys that present yield-strength anomaly have been overcome by proposing a modified version that describes the flow-stress dependence on temperature through the superposition of two terms. The first term accounts for the general response of the alloy (plastic-strain hardening and thermal softening) while the second term models the peak-flow stress in the desired temperature range through a phenomenological expression. The proposed model was satisfactorily calibrated for three alloys, a cobaltbase superalloy, a maraging steel and a DS nickel-base superalloy, with quite different evolutions of the flow-strength with temperature, demonstrating its adaptability.

The proposed model is transferable to the industry and/or can be easily used by other researchers since its implementation in a commercial finiteelement code and constants calibration are not tedious.

- The CPFEM-strategy employed was accurate enough to predict with high fidelity the anisotropic response of the DS nickel-base superalloy MARM247 at different strain rates and room temperature. Taking advantage 
of the oligocrystalline nature of the specimens, the numerical strategy was based on simulating the whole gauge length of the specimens with a crystal plasticity model. Slip on octahedral systems was only assumed and the response of each crystal was formulated with phenomenological laws, although dislocation-dislocation interactions and junctions were considered.

The simulations displayed that the first stages of plastic deformation tend to be accommodated by grains with higher Schmid factors. However, the rest of grains increased their importance to accommodate plastic deformation in the final stages. Moreover, the analysis of the local fields showed that grains oriented perpendicularly to the loading axis are nucleation sites of voids due to the pronounced local-stress increase factor.

The capacity of simulation framework to be used as a tool for virtual testing was also demonstrated. The experimental true stress-strain curves for $90^{\circ}$-oriented samples and mechanical constants, such as the $0.2 \%$ yield strength, Young's modulus and Poisson's ratio, of a quasi-polycrystal and a polycrystal version of the alloy were well predicted by using constants that were only fitted with data from $0^{\circ}$-oriented samples.

\section{$5.2 \quad$ Future work}

The next research lines are proposed to continue the work presented in this thesis.

- Some of the materials with yield-strength anomaly present an important anisotropic behaviour due to its microstructure, so the implementation of the proposed phenomenological model by using an anisotropic yield function would be interesting. Moreover, another improvement would be based on considering the effect of strain rate on the temperature at which the flow stress peak appears and on the shape of that region. To that end, a new experimental campaign should be done in the quasi-static and intermediate-rate regime. 


\subsection{Future work}

- Given the large grain sizes of the DS Ni-base superalloy, the research could continue by measuring the strain field at the grain levels during quasi-static and dynamic experiments. Then, this information could be compared with the strain field obtained from CPFEM simulations of the real microstructure of the sample. Taking advantage of the columnar structure, the virtual sample could be reconstructed from the EBSD data of a cross-section perpendicular to the grain-growth direction.

- Following with the CP model, the formulation could be updated to consider the cross-slip pinning mechanism and cube slip at high temperatures and simulate the split-Hopkinson tension bar experiments performed on MAR-M247 DS above room temperature. Moreover, the temperature increment due to adiabatic heating should be added.

- Finally, introducing a failure criterion in the CPFEM framework would be also an interesting task. 


\section{Appendix $\Lambda$}

\section{Notes on continuum mechanics}

This appendix contains only basic notes on continuum mechanics. For more in-depth information, look up in [28].

Let be two regions $\Omega_{0}$ and $\Omega_{t}$, occupied by a body $\mathcal{B}$ in the reference or current $\left({ }_{0}\right)$ and deformed $(t)$ configuration, and the vectors $\mathbf{x}$ and $\mathbf{y}$ that define, respectively, the material points in the reference and deformed configuration (Figure A.1), the deformation gradient $\mathbf{F}$ is defined as:

$$
\mathbf{F}=\frac{\partial \mathbf{y}}{\partial \mathbf{x}}=\mathbf{I}+\frac{\partial \mathbf{u}}{\partial \mathbf{x}}
$$

where $\mathbf{u}=\mathbf{y}-\mathbf{x}$ is the displacement of any point between the deformed and reference configuration.

The deformation gradient $\mathbf{F}$ contains the information about the rotations and deformations suffered by the body $\mathcal{B}$ between the reference and deformed configuration, and by using the polar decomposition, can be written as:

$$
\mathbf{F}=\mathbf{R U}=\mathbf{V R}
$$

where the rotations are defined by the rotation tensor $\mathbf{R}$ and $\mathbf{U}$ and $\mathbf{V}$ are, respectively, the right and left stretch tensors that contain all information about the deformations. $\mathbf{U}$ defines the deformation with respect to the reference configuration while $\mathbf{V}$ defines the deformation with respect to the rotated configuration (see Figure A.1). 


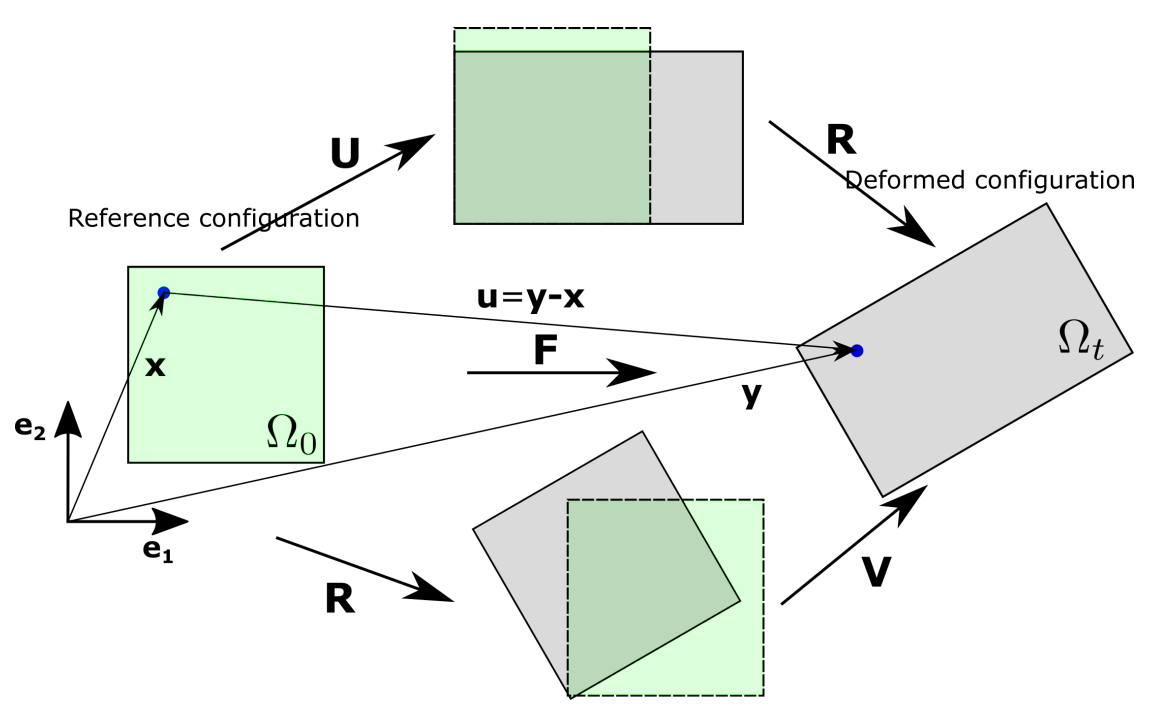

Figure A.1: Reference and deformed configuration. Graphic representation of the polar decomposition of the deformation gradient.

Similarly, the velocity gradient $\mathbf{L}=\frac{\partial \dot{\mathbf{u}}}{\partial \mathbf{y}}=\frac{\partial \mathbf{v}}{\partial \mathbf{y}}=\dot{\mathbf{F}} \mathbf{F}^{-1}$, which describes the relative velocity between two material points in the deformed configuration, can be decomposed as:

$$
\mathbf{L}=\mathbf{D}+\mathbf{W}
$$

where $\mathbf{D}$ is the rate of deformation tensor (information about deformations in the rotated configuration) and $\mathbf{W}$ the spin tensor (information about rotations).

\section{A.1 Stresses and strains definitions}

Let be $\boldsymbol{\sigma}$ the Cauchy stress tensor (deformed configuration), the second Piola-Kirchhoff tensor $\mathbf{S}$ and its conjugate $\mathbf{E}^{\mathbf{e}}$ Green-Lagrange strain tensor, both in the reference configuration, can be written as:

$$
\begin{aligned}
& \mathbf{S}=J \mathbf{F}^{-1} \boldsymbol{\sigma} \mathbf{F}^{-T}=\operatorname{det}(\mathbf{F}) \mathbf{F}^{-1} \boldsymbol{\sigma} \mathbf{F}^{-T} \\
& \mathbf{E}^{\mathbf{e}}=\frac{1}{2}\left(\mathbf{F}^{T} \mathbf{F}-\mathbf{I}\right)=\frac{1}{2}\left(\mathbf{U}^{T} \mathbf{U}-\mathbf{I}\right)
\end{aligned}
$$




\section{${ }_{\text {APpendix }} \mathrm{B}$}

\section{Euler angles}

The Euler angles are a set of three angles that are used to represent the orientation of a 3D rigid body with respect to a reference coordinate system $\left(\mathbf{e}_{x}\right.$, $\mathbf{e}_{y}, \mathbf{e}_{z}$ ). Each angle corresponds to a rotation about different axes. Depending on the axes about which the rotations are conducted, there are different conventions. In materials science, the crystallographic orientation is usually detailed according to the Bunge convention [111]. In this convention, the Euler angles $\left(\phi_{1}, \Phi, \phi_{2}\right)$, which define the crystal coordinate system $\left(\mathbf{e}_{x}^{c}, \mathbf{e}_{y}^{c}, \mathbf{e}_{z}^{c}\right)$ with respect to the reference system $\left(\mathbf{e}_{x}, \mathbf{e}_{y}, \mathbf{e}_{z}\right)$, stand for (see Figure B.1):

1. $\phi_{1}$ (radian or degree) rotation about $\mathbf{e}_{z} .\left(\mathbf{e}_{x}, \mathbf{e}_{y}, \mathbf{e}_{z}\right) \rightarrow\left(\mathbf{e}_{x}^{\prime}, \mathbf{e}_{y}^{\prime}, \mathbf{e}_{z}^{\prime}\right)$

2. $\Phi$ (radian or degree) rotation about $\mathbf{e}_{x}^{\prime} \cdot\left(\mathbf{e}_{x}^{\prime}, \mathbf{e}_{y}^{\prime}, \mathbf{e}_{z}^{\prime}\right) \rightarrow\left(\mathbf{e}_{x}^{\prime \prime}, \mathbf{e}_{y}^{\prime \prime}, \mathbf{e}_{z}^{\prime \prime}\right)$

3. $\phi_{2}$ (radian or degree) rotation about $\mathbf{e}_{z}^{\prime \prime} \cdot\left(\mathbf{e}_{x}^{\prime \prime}, \mathbf{e}_{y}^{\prime \prime}, \mathbf{e}_{z}^{\prime \prime}\right) \rightarrow\left(\mathbf{e}_{x}^{c}, \mathbf{e}_{y}^{c}, \mathbf{e}_{z}^{c}\right)$

Renaming the set of angles $\left(\phi_{1}, \Phi, \phi_{2}\right)$ to $(1,2,3)$ and the trigonometric functions (cos, $\sin )$ to $(c, s)$, the transformation matrix $\mathbf{Q}^{\theta}$ is written as:

$$
\mathbf{Q}^{\theta}\left(\phi_{1}, \Phi, \phi_{2}\right)=\left(\begin{array}{ccc}
c 1 c 3-c 2 s 1 s 3 & c 3 s 1+c 2 c 1 s 3 & s 3 s 2 \\
-c 1 s 3-c 3 c 2 s 1 & c 1 c 2 c 3-s 1 s 3 & c 3 s 2 \\
s 2 s 1 & -c 1 s 2 & c 2
\end{array}\right)
$$


Given a tensor defined in the reference configuration, the transformation matrix $\mathbf{Q}^{\theta}$ express that tensor in the rotated configuration after applying the corresponding operations:

$$
\begin{gathered}
\mathbf{u}^{c}=\mathbf{Q}^{\theta} \cdot \mathbf{u} \\
\mathbf{A}^{c}=\mathbf{Q}^{\theta} \cdot \mathbf{A} \cdot\left(\mathbf{Q}^{\theta}\right)^{T} \\
\mathbb{A}^{c}=\mathbf{Q}^{\theta} \cdot \mathbf{Q}^{\theta} \cdot \mathbb{A} \cdot\left(\mathbf{Q}^{\theta}\right)^{T} \cdot\left(\mathbf{Q}^{\theta}\right)^{T}
\end{gathered}
$$

The columns of the transformation matrix represent the axes of the reference configuration in the rotated configuration, while the rows stand for the axes of the rotated configuration in the reference configuration.
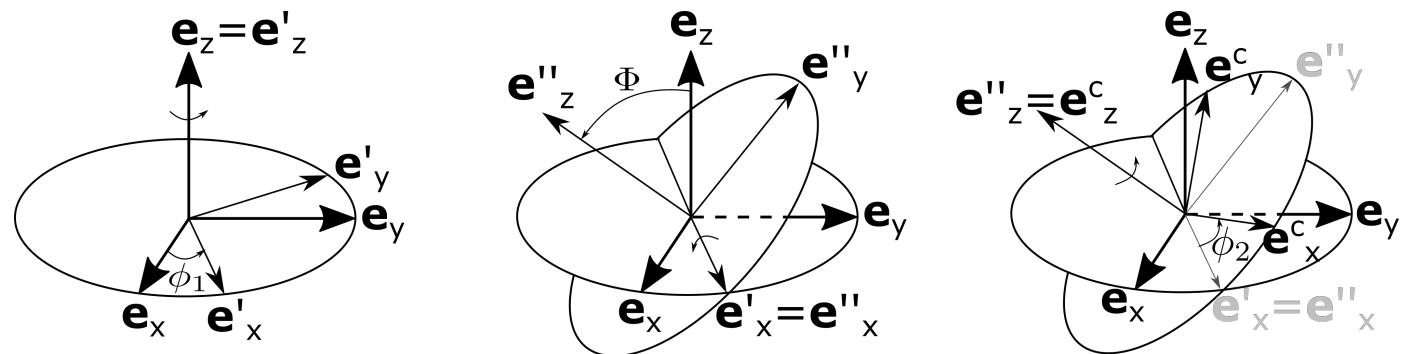

Figure B.1: Axes rotations defined by Euler angles in Bunge convention. 


\section{Appendix}

\section{Mesh-size sensitivity}

A mesh-size sensitivity analysis for the crystal-plasticity simulations is presented below. The response of a $90^{\circ}$ specimen under quasi-static tensile conditions was simulated with three different element sizes $\left(0.3 \times 0.3 \times 0.3 \mathrm{~mm}^{3}\right.$, $0.2 \times 0.2 \times 0.2 \mathrm{~mm}^{3}$ and $0.15 \times 0.15 \times 0.15 \mathrm{~mm}^{3}$ ) (Figure C.1) but keeping the grain structure and orientations. For the analysis, the $90^{\circ}$-specimen configuration was chosen over the $0^{\circ}$-specimen configuration because the microstructure is more heterogeneous. The material model and procedure was the same as those used in Chapter 4. The finite-element simulation of the sample meshed with 0.3 -mm-elements took $3989 \mathrm{~s}$, while the simulations of the samples with 0.2- and 0.15-mm-elements took, respectively, $10906 \mathrm{~s}$ and $29133 \mathrm{~s}$ using ( $8 \mathrm{x}$ Intel (R) Xeon (R) CPU E5-2640 v4 @ 2.40 GHz).

The true stress-strain curves for the virtual specimen with different sizes of elements are plotted in Figure C.2 and show that the macroscopic response is not sensitive to the mesh sizes studied. However, the local fields, such as the local von Mises stress for each element (see Figure C.3), are sensitive, to some degree, to the element size and therefore, fine meshes are needed to capture fields concentration on grain boundaries (see Figure C.4). 


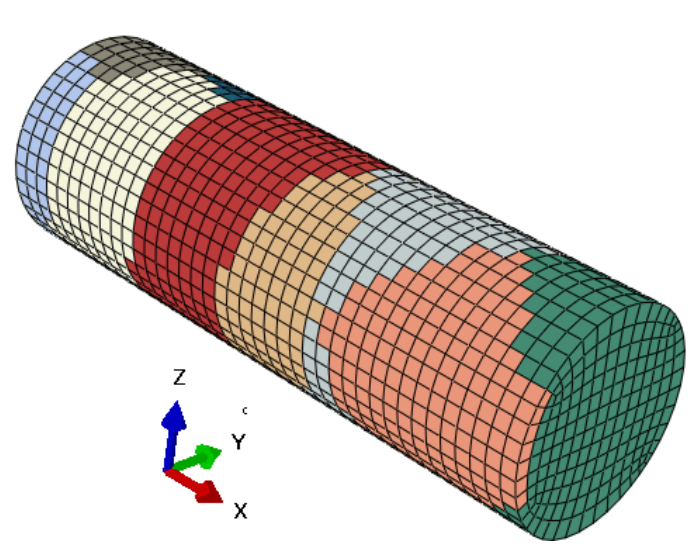

(a)

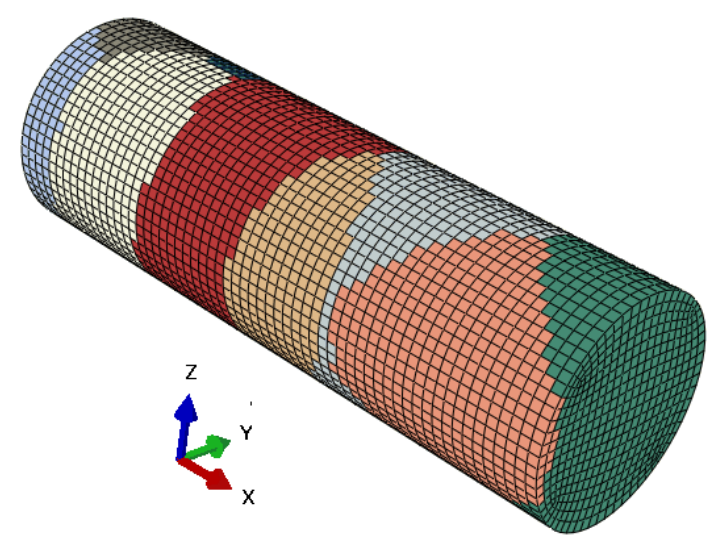

(b)

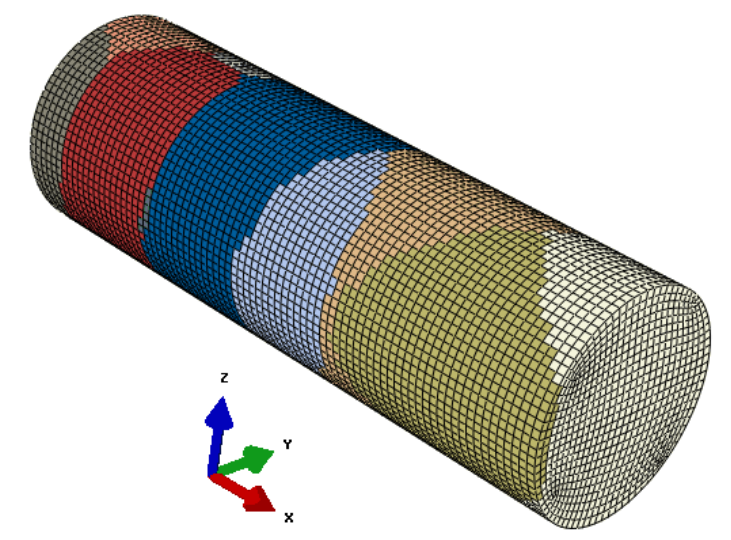

(c)

Figure C.1: $90^{\circ}$ samples with different element sizes. (a) $0.3 \times 0.3 \times 0.3$ $\mathrm{mm}^{3}$, (b) $0.2 \times 0.2 \times 0.2 \mathrm{~mm}^{3}$ and (c) $0.15 \times 0.15 \times 0.15 \mathrm{~mm}^{3}$. 


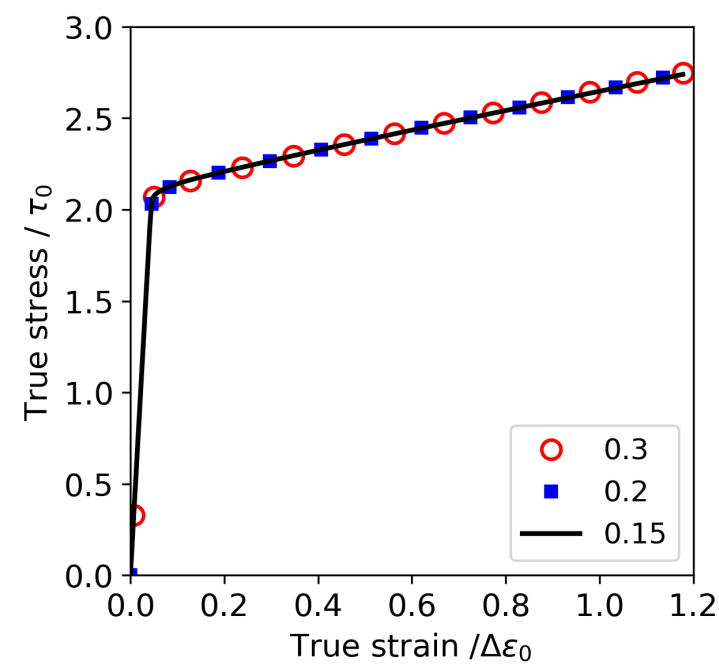

Figure C.2: True stress-strain curves for the same virtual specimen with different size of elements. $0.3 \times 0.3 \times 0.3 \mathrm{~mm}^{3}, 0.2 \times 0.2 \times 0.2 \mathrm{~mm}^{3}$ and 0.15 x $0.15 \times 0.15 \mathrm{~mm}^{3}$

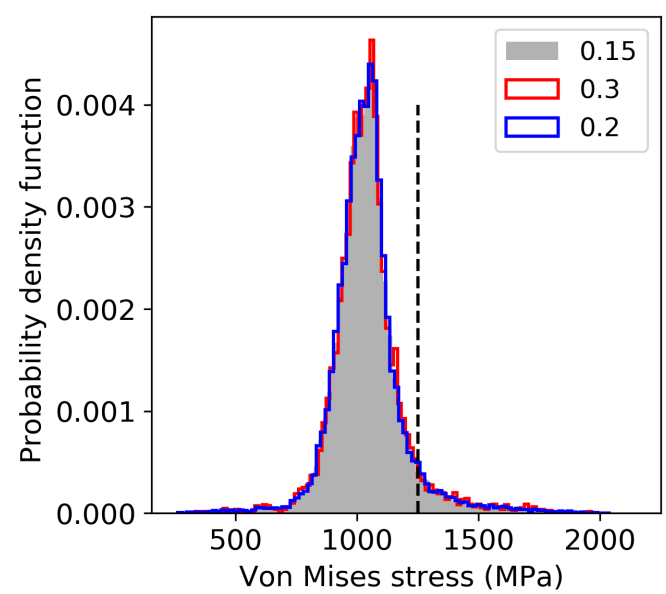

(a)

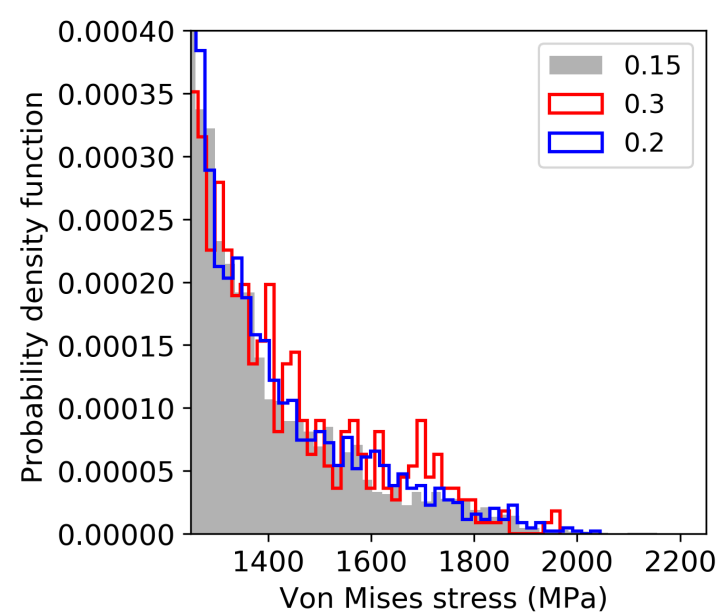

(b)

Figure C.3: Histogram representing the relative amount of elements with a certain level of local von Mises stress in the last frame of the simulation. (a) General plot and (b) same plot with a zoom-in for values larger than 1250 MPa (dashed line in (a)). 


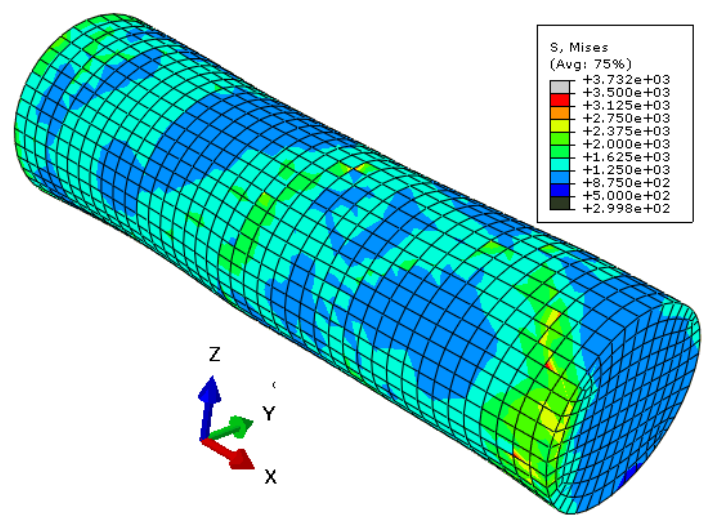

(a)

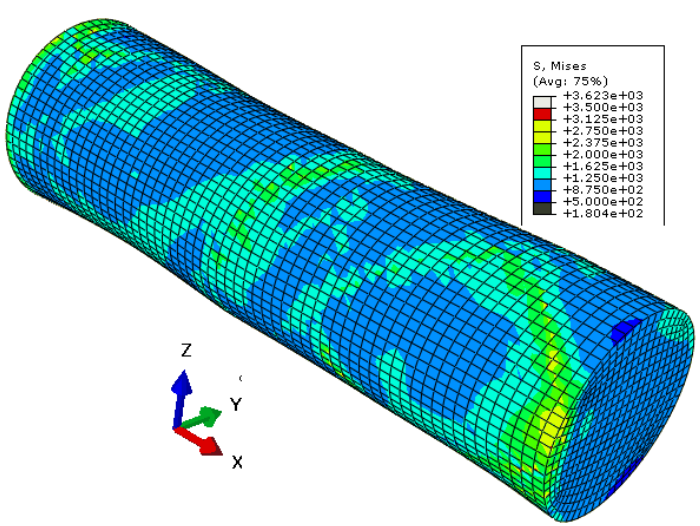

(b)

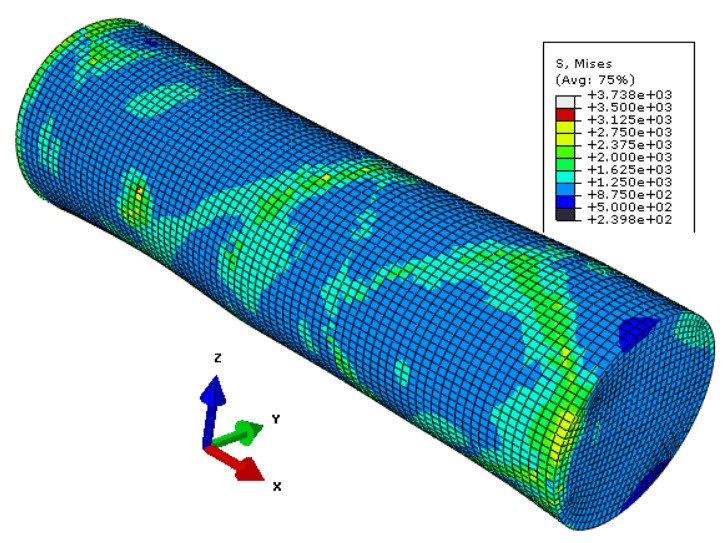

(c)

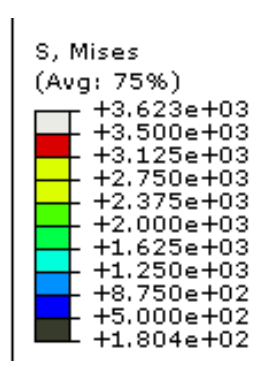

(d)

Figure C.4: Distribution of the von-Mises stress (MPa) on $90^{\circ}$ samples in the last frame of the simulation. (a) $0.3 \times 0.3 \times 0.3 \mathrm{~mm}^{3}$, (b) $0.2 \times 0.2 \times 0.2$ $\mathrm{mm}^{3}$ and (c) $0.15 \times 0.15 \times 0.15 \mathrm{~mm}^{3}$. 


\section{${ }_{\text {APpendx }} \mathrm{D}$}

\section{Implicit dymamic analysis}

In the finite element method, the equation of motion of a dynamic problem (equation D.1, where $M$ is the discrete mass, $\mathbf{u}$ the nodal displacement, $\mathbf{f}^{\text {ext }}$ the applied load and $\mathbf{f}^{\text {int }}$ the internal forces of the structure) can be solved by using either an explicit or an implicit time-integration procedure. However, the explicit option is highly recommended in wave-propagation problems [112].

$$
M \ddot{\mathbf{u}}=\mathbf{f}^{e x t}-\mathbf{f}^{\text {int }}
$$

In the present thesis, with the aim of using the same crystal-plasticity subroutine for both the quasi-static and dynamic tests, the split-Hopkinson bar tests were simulated, in Chapter 4, by employing the implicit dynamic solver implemented in Abaqus [66]. Although Abaqus estimates the time step depending on whether we label the problem as a transient fidelity application, moderate dissipation application (our case ${ }^{1}$ ) or quasi-static application, important differences between the explicit ("correct") and implicit solution may appear during wave propagation. Below is an analysis of these differences on a cubic sample, with size of $10 \times 10 \times 10 \mathrm{~mm}^{3}$, subjected to SHPB-like boundary conditions. The sample (Figure D.1) was meshed by using 40 CRD8 elements along each direction and the material was defined with a Young's modulus of $\mathrm{E}=200$ GPa, poisson's ratio $\nu=0.3$, density $\rho=8000 \mathrm{~kg} / \mathrm{m}^{3}$ and a perfectly plastic

\footnotetext{
${ }^{1}$ Impact analysis on a metallic material that dissipates energy by plastic deformation
} 
behaviour $Y\left(\bar{\varepsilon}_{p}\right)=1 \mathrm{GPa}$. The SHPB-like boundary conditions were set by imposing a trapezoid-shaped velocity profile, with a rise time of $0.02 \mathrm{~ms}$ and a maximum velocity of $v_{y}=10 \mathrm{~m} / \mathrm{s}$, to the nodes on the plane $y=0$ and avoiding normal displacement on $x=0, y=L$ and $z=0$. It is important to note that any other set of mechanical constants (similar to those of metallic materials) could be used.

The explicit analysis was run with the default values proposed by Abaqus. On the other hand, the dynamic implicit analysis, using the moderate dissipation option, was run with two different maximum time steps: $\Delta t=0.01 \mathrm{~ms}$ (200 times larger than the Courant number) and $\Delta t=5 \cdot 10^{-4} \mathrm{~ms}$ (10 times larger than the Courant number). The study shows that a large time increment is accurate enough to predict the reaction forces developed by the sample (Figure D.2) during the plastic regime. However, the elastic and the elastic-to-plastic transition region is not well reproduced and smaller time increments must be employed in those regions. In more detail, Figures D.3 to D.8 compare the stress evolution, along $y$-direction, during the elastic loading region (red area) calculated by the explicit (black lines) and the dynamic implicit (blue lines) solver with the time increment of $\Delta t=5 \cdot 10^{-4} \mathrm{~ms}$. The data were obtained from the elements in the middle of the corresponding $y$ plane. Initially there are important errors between both methods but the solutions converges on comparable values while achieving stress equilibrium.

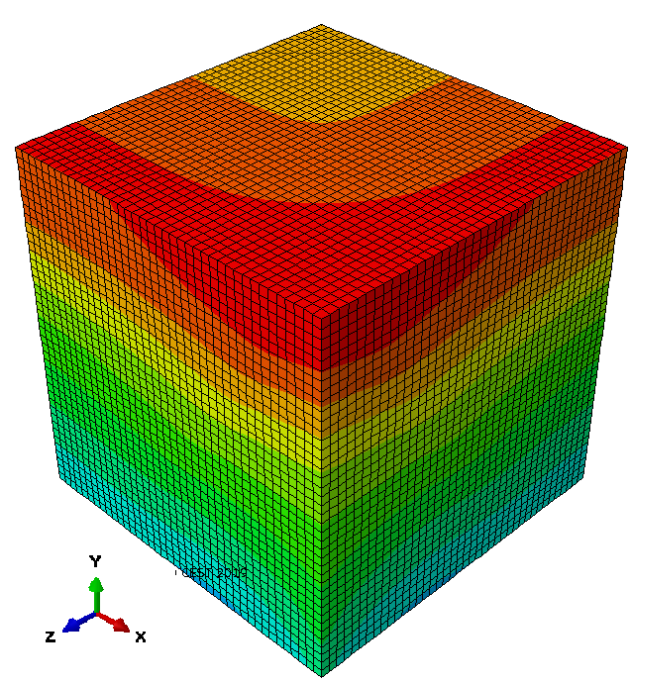

Figure D.1: Sample 


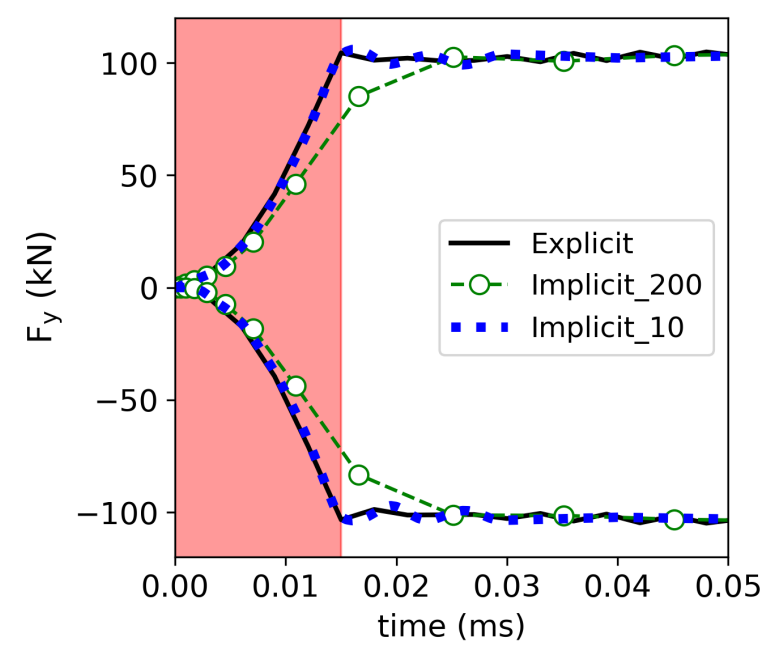

Figure D.2: Reaction forces ( $y$ - component) versus time history on sample surfaces $y=0$ and $y=L$ using the explicit (black line) and dynamic implicit solver.

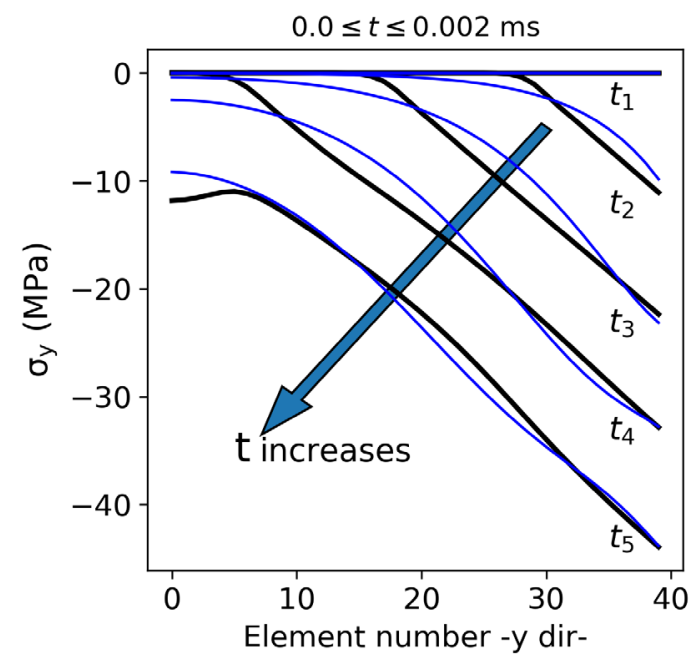

(a)

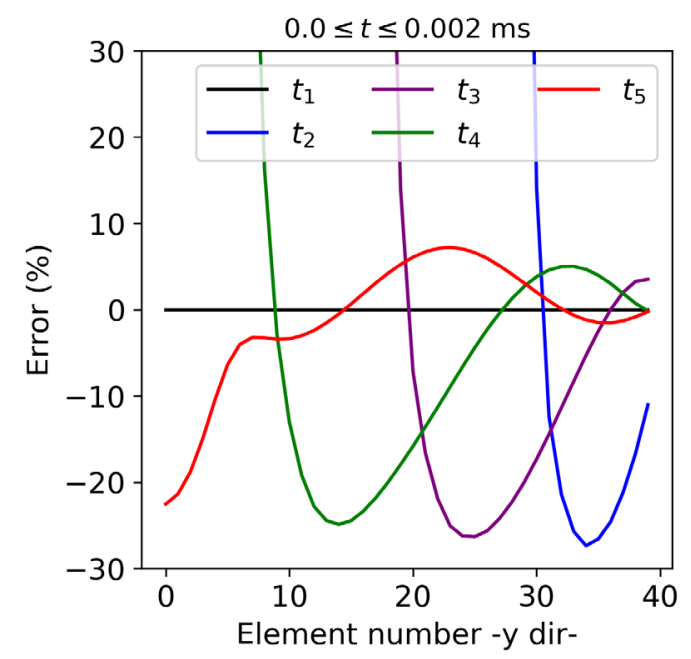

(b)

Figure D.3: (a) Stress evolution ( $y$ - component) along y-direction of the sample (loading direction) during the elastic loading for $0.0 \leq 0.002 \mathrm{~ms}$. Black lines correspond to the explicit solver while blue lines to the implicit one. (b) Difference (in \%) between the implicit and explicit solution. 


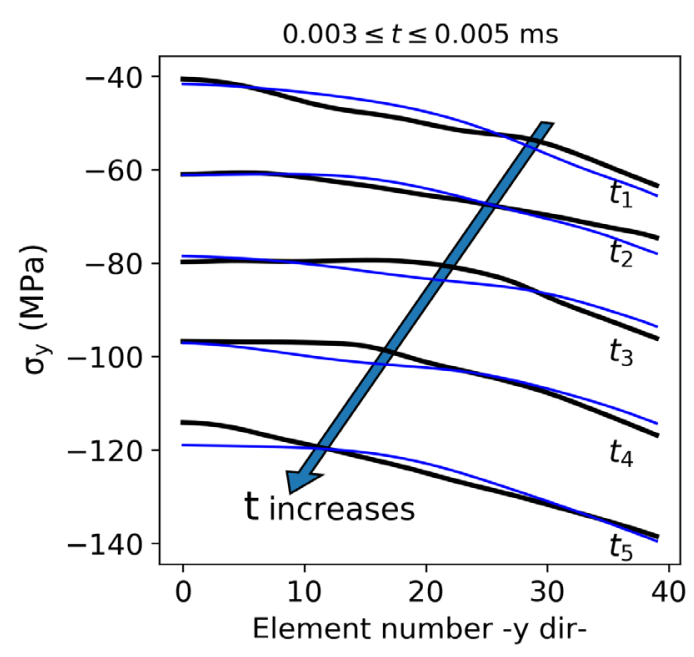

(a)

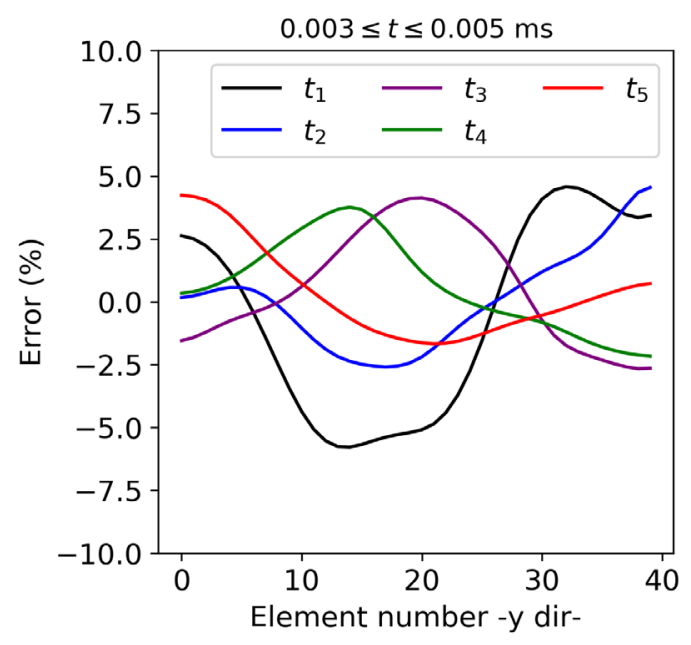

(b)

Figure D.4: a) Stress evolution ( $y$-component) along y-direction of the sample (loading direction) during the elastic loading for $0.003 \leq 0.005 \mathrm{~ms}$. Black lines correspond to the explicit solver while blue lines to the implicit one. b) Difference (in \%) between the implicit and explicit solution.

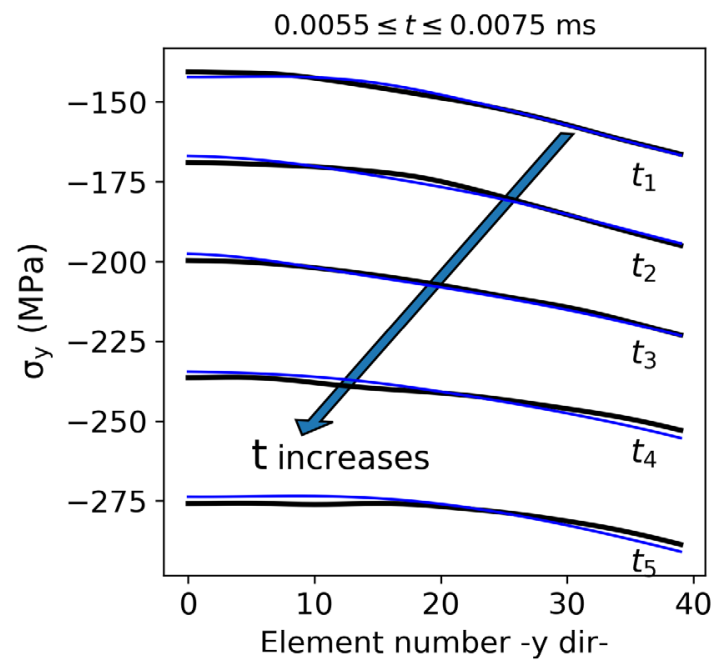

(a)

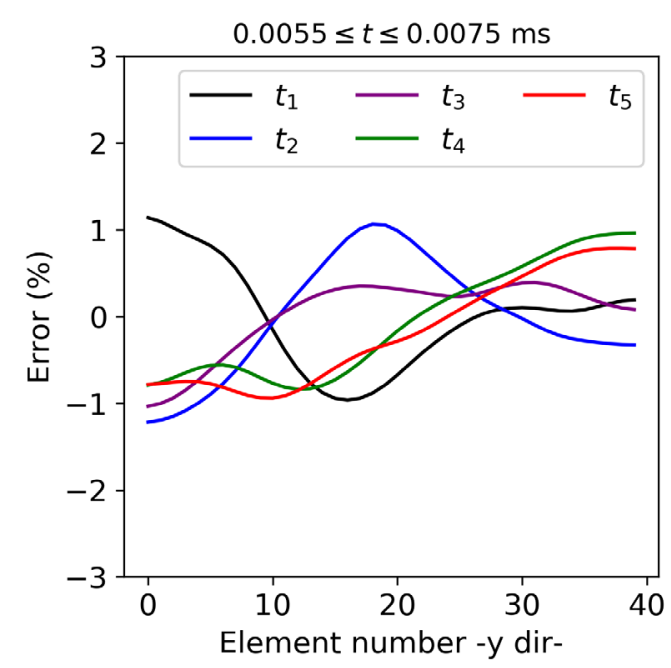

(b)

Figure D.5: a) Stress evolution ( $y$-component) along loading direction for $0.0055 \leq 0.0075 \mathrm{~ms}$. Black lines correspond to the explicit solver while blue lines to the implicit one. b) Difference (in \%) between the implicit and explicit solution. 


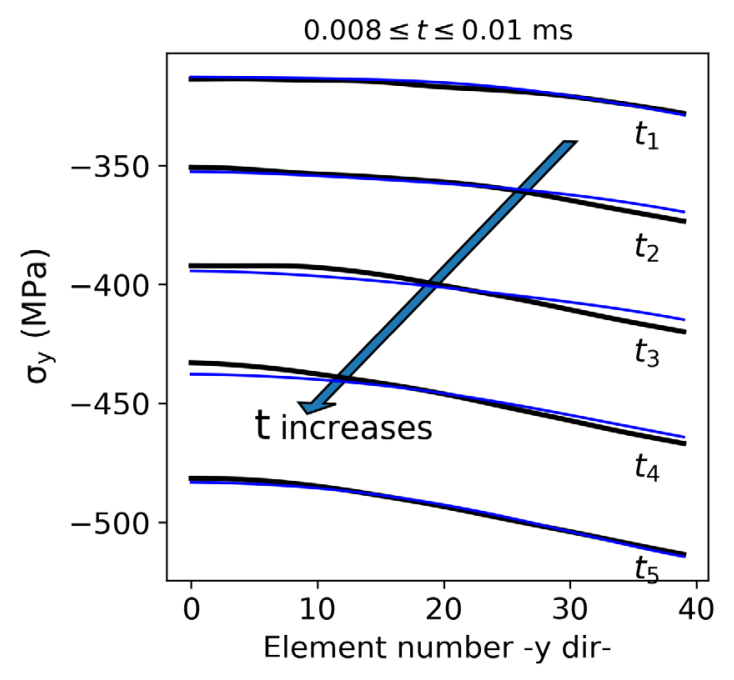

(a)

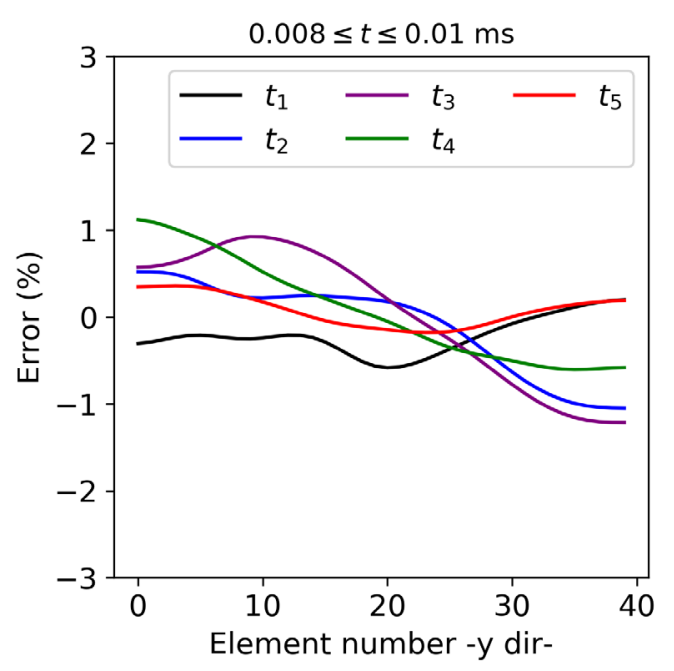

(b)

Figure D.6: a) Stress evolution ( $y$-component) along loading direction for $0.008 \leq 0.01 \mathrm{~ms}$. Black lines correspond to the explicit solver while blue lines to the implicit one. b) Difference (in \%) between the implicit and explicit solution.

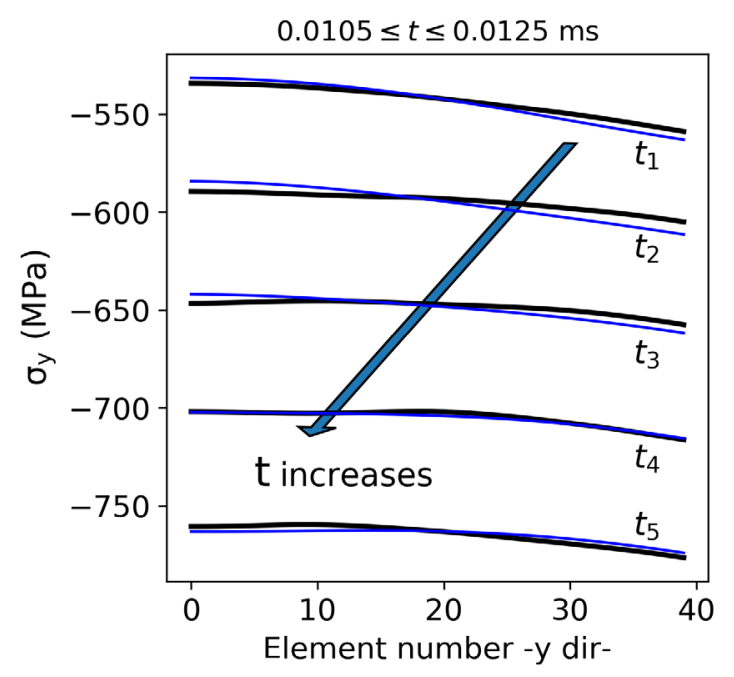

(a)

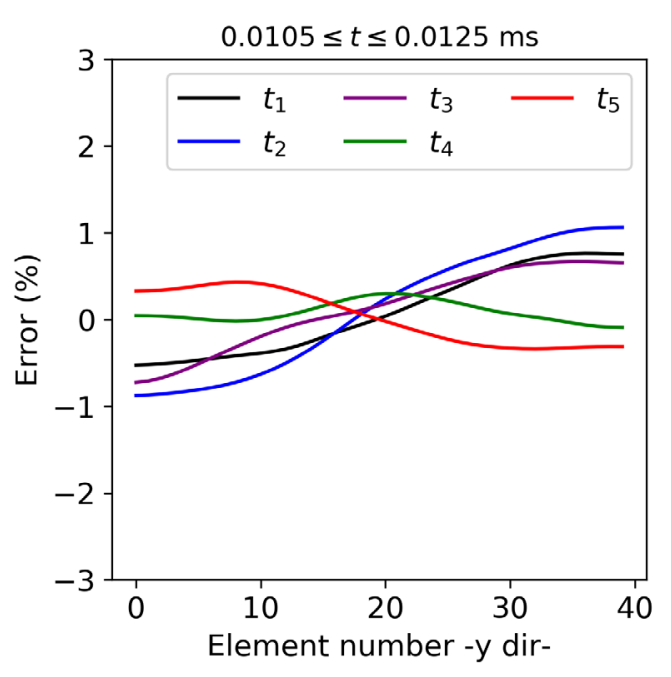

(b)

Figure D.7: a) Stress evolution ( $y$-component) along loading direction for $0.0105 \leq 0.0125 \mathrm{~ms}$. Black lines correspond to the explicit solver while blue lines to the implicit one. b) Difference (in \%) between the implicit and explicit solution. 


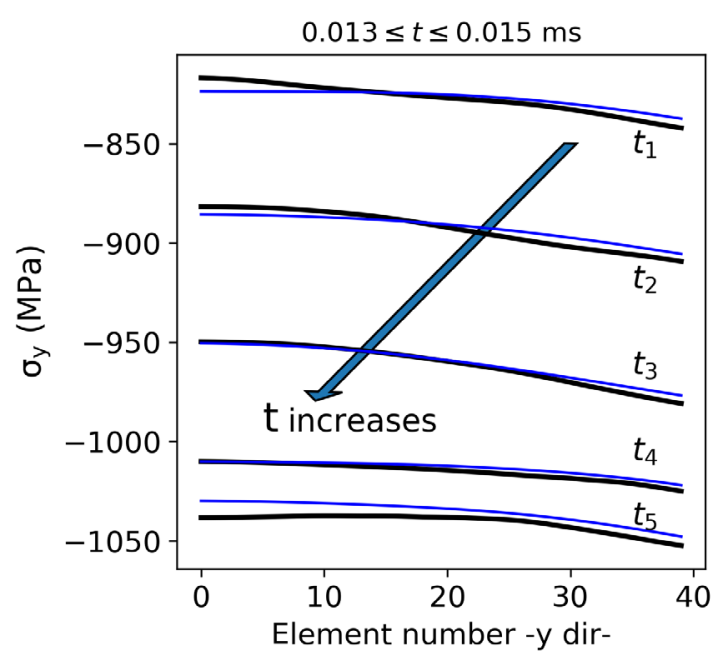

(a)

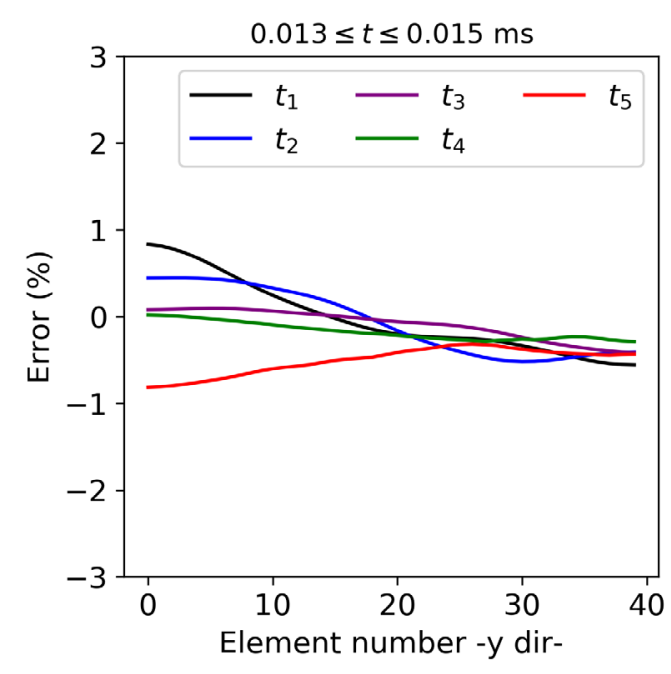

(b)

Figure D.8: a) Stress evolution ( $y$ - component) along loading direction for $0.013 \leq 0.015 \mathrm{~ms}$. Black lines correspond to the explicit solver while blue lines to the implicit one. b) Difference (in \%) between the implicit and explicit solution. . 


\section{Bibliography}

[1] Yan Zhang and Mark E. Weber. Radar and aerospace [special issue]. Aerospace, 4(1), 2017.

[2] G Riegert, K Pfleiderer, H Gerhard, I Solodov, and G Busse. Modern methods of NDT for inspection of aerospace structures. In ECNDT: Berlin, Germany, 2006.

[3] Armonización de las actividades en materiales compuestos para aeronáutica y espacio en españa. https://plataforma-aeroespacial. es/wp-content/uploads/2017/09/materiales_compuestos.pdf. Accessed: 2020-07-01.

[4] Turbofan operation. https://commons.wikimedia.org/wiki/File: Turbofan_operation.svg. Accessed: 2020-04-01.

[5] Rolls Royce. The jet engine. John Wiley \& Sons, Derby, England, 2015.

[6] Akane Suzuki, Garret C DeNolf, and Tresa M Pollock. Flow stress anomalies in $\gamma-\gamma^{\prime}$ two-phase co-al-w-base alloys. Scripta Materialia, 56(5):385388, 2007.

[7] D Mukherji, J Rosler, J Wehrs, H Eckerlebe, and R Gilles. Co-Re-based alloys a new class of material for gas turbine applications at very high temperatures. Advances in materials Research, 1(3):205-219, 2012.

[8] Xi Chen and John W Hutchinson. Particle impact on metal substrates with application to foreign object damage to aircraft engines. Journal of the Mechanics and Physics of Solids, 50(12):2669-2690, 2002. 
[9] Zdzislaw Mazur, Alberto Luna-Ramirez, JA Juárez-Islas, and A CamposAmezcua. Failure analysis of a gas turbine blade made of inconel 738lc alloy. Engineering failure analysis, 12(3):474-486, 2005.

[10] B Erice, F Gálvez, DA Cendón, V Sánchez-Gálvez, and T Børvik. An experimental and numerical study of ballistic impacts on a turbine casing material at varying temperatures. Journal of applied mechanics, 78(5), 2011.

[11] Gordon R Johnson and William H Cook. A constitutive model and data for metals subjected to large strains, high strain rates and high temperatures. In Proceedings of the 7th International Symposium on Ballistics, volume 21, pages 541-547. The Netherlands, 1983.

[12] Sidney Chocron, Borja Erice, and Charles E Anderson. A new plasticity and failure model for ballistic application. International Journal of Impact Engineering, 38(8-9):755-764, 2011.

[13] Borja Erice Echávarri. Flow and Fracture Behaviour of High Performance Alloys. PhD thesis, Ph. D. Tesis, Universidad Politécnica de Madrid, 2012.

[14] Christian C. Roth and Dirk Mohr. Effect of strain rate on ductile fracture initiation in advanced high strength steel sheets: Experiments and modeling. International Journal of Plasticity, 56:19-44, 2014.

[15] Jiao Liu, Bailin Zheng, Kai Zhang, Biao Yang, and Xiaoqiang Yu. Ballistic performance and energy absorption characteristics of thin nickel-based alloy plates at elevated temperatures. International Journal of Impact Engineering, 126:160-171, 2019.

[16] M. M. Shenoy, D. L. McDowell, and R. W. Neu. Transversely isotropic viscoplasticity model for a directionally solidified Ni-base superalloy. International Journal of Plasticity, 22(12):2301-2326, 2006.

[17] Duoqi Shi, Chengli Dong, and Xiaoguang Yang. Constitutive modeling and failure mechanisms of anisotropic tensile and creep behaviors of nickel- 


\section{BIBLIOGRAPHY}

base directionally solidified superalloy. Materials and Design, 45:663-673, 2013 .

[18] Hafid Sabar, Marcel Berveiller, Veronique Favier, and Stephane Berbenni. A new class of micro-macro models for elastic-viscoplastic heterogeneous materials. International Journal of Solids and Structures, 39(12):3257$3276,2002$.

[19] G. Martin, N. Ochoa, K. Saï, E. Hervé-Luanco, and G. Cailletaud. A multiscale model for the elastoviscoplastic behavior of Directionally Solidified alloys: Application to FE structural computations. International Journal of Solids and Structures, 51(5):1175-1187, 2014.

[20] R. J. Kashinga, L. G. Zhao, V. V. Silberschmidt, F. Farukh, N. C. Barnard, M. T. Whittaker, D. Proprentner, B. Shollock, and G. McColvin. Low cycle fatigue of a directionally solidified nickel-based superalloy: Testing, characterisation and modelling. Materials Science and Engineering A, 708:503-513, 2017.

[21] B. Lin, L. G. Zhao, and J. Tong. A crystal plasticity study of cyclic constitutive behaviour, crack-tip deformation and crack-growth path for a polycrystalline nickel-based superalloy. Engineering Fracture Mechanics, 78(10):2174-2192, 2011.

[22] Yongjun Guan, Bo Chen, Jinwen Zou, T. Ben Britton, Jun Jiang, and Fionn P.E. Dunne. Crystal plasticity modelling and HR-DIC measurement of slip activation and strain localization in single and oligo-crystal $\mathrm{Ni}$ alloys under fatigue. International Journal of Plasticity, 88:70-88, 2017.

[23] Marko Knezevic, John S. Carpenter, Manuel L. Lovato, and Rodney J. McCabe. Deformation behavior of the cobalt-based superalloy Haynes 25: Experimental characterization and crystal plasticity modeling. Acta Materialia, 63:162-168, 2014.

[24] Ahmad Shahba and Somnath Ghosh. Crystal plasticity FE modeling of 
Ti alloys for a range of strain-rates. Part I: A unified constitutive model and flow rule. International Journal of Plasticity, 87:48-68, 2016.

[25] Somnath Ghosh, Ahmad Shahba, Xiaohui Tu, Emily L. Huskins, and Brian E. Schuster. Crystal plasticity FE modeling of Ti alloys for a range of strain-rates. Part II: Image-based model with experimental validation. International Journal of Plasticity, 87:69-85, 2016.

[26] William D Callister and David G Rethwisch. Materials science and engineering: an introduction, volume 9. Wiley New York, 2018.

[27] Derek Hull and David J Bacon. Introduction to dislocations, volume 37. Elsevier, 2011.

[28] Eduardo A de Souza Neto, Djordje Peric, and David RJ Owen. Computational methods for plasticity: theory and applications. John Wiley \& Sons, 2011.

[29] Javier Segurado, Ricardo A. Lebensohn, and Javier LLorca. Chapter one - computational homogenization of polycrystals. In Mahmoud I. Hussein, editor, Advances in Crystals and Elastic Metamaterials, Part 1, volume 51 of Advances in Applied Mechanics, pages 1 - 114. Elsevier, 2018.

[30] V Tvergaard and A Needleman. Effect of crack meandering on dynamic, ductile fracture. Journal of the Mechanics and Physics of Solids, 40(2):447-471, 1992.

[31] R von Mises. Mechanik der festen körper im plastisch-deformablen zustand. Nachrichten von der Gesellschaft der Wissenschaften zu Göttingen, Mathematisch-Physikalische Klasse, 1913:582-592, 1913.

[32] EH Lee and DT Liu. Finite-strain elastic - plastic theory with application to plane-wave analysis. Journal of applied physics, 38(1):19-27, 1967.

[33] James R Rice. Inelastic constitutive relations for solids: an internalvariable theory and its application to metal plasticity. Journal of the Mechanics and Physics of Solids, 19(6):433-455, 1971. 


\section{BIBLIOGRAPHY}

[34] Geoffrey Ingram Taylor. Plastic strain in metals. J. Inst. Metals, 62:307324, 1938.

[35] Georg Sachs. Zur ableitung einer fliessbedingung. In Mitteilungen der deutschen Materialprüfungsanstalten, pages 94-97. Springer, 1929.

[36] JD Eshelby. Proceedings of the royal society of london a: Mathematical, physical and engineering sciences. 1957.

[37] Ricardo A Lebensohn and CN Tomé. A self-consistent anisotropic approach for the simulation of plastic deformation and texture development of polycrystals: application to zirconium alloys. Acta metallurgica et materialia, 41(9):2611-2624, 1993.

[38] Javier Segurado, Ricardo A Lebensohn, Javier LLorca, and Carlos N Tomé. Multiscale modeling of plasticity based on embedding the viscoplastic self-consistent formulation in implicit finite elements. International Journal of Plasticity, 28(1):124-140, 2012.

[39] Javier Segurado and Javier Llorca. Simulation of the deformation of polycrystalline nanostructured Ti by computational homogenization. Computational materials science, 76:3-11, 2013.

[40] GT Camacho and M Ortiz. Adaptive lagrangian modelling of ballistic penetration of metallic targets. Computer methods in applied mechanics and engineering, 142(3-4):269-301, 1997.

[41] T Børvik, OS Hopperstad, T Berstad, and M Langseth. A computational model of viscoplasticity and ductile damage for impact and penetration. European Journal of Mechanics-A/Solids, 20(5):685-712, 2001.

[42] E Voce. The relationship between stress and strain for homogeneous deformation. Journal of the Institute of Metals, 74:537-562, 1948.

[43] D. P. Pope. Mechanical properties of $\mathrm{Ni}_{3} \mathrm{Al}$ and nickel-base alloys with high volume fraction of $\gamma^{\prime}$. International Metals Reviews, 29(1):136-167, 1984. 
[44] Eckhard Nembach. The high temperature peak of the yield strength of $\gamma^{\prime}$-strengthened superalloys. Materials Science and Engineering A, 429(12):277-286, 2006.

[45] Paul A Flinn. Theory of deformation in superlattices. Transactions of the American Institute of Mining and Metallurgical Engineers, 218(1):145$154,1960$.

[46] BH Kear and HG Wilsdorf. Dislocation configurations in plastically deformed polycrystalline $\mathrm{Cu}_{3} \mathrm{Au}$ crystals. Transactions of the American Institute of Mining and Metallurgical Engineers, 224(1):382-386, 1962.

[47] BH Kear. Dislocation configurations and work hardening in $\mathrm{Cu}_{3} \mathrm{Au}$ crystals. Acta Metallurgica, 12(5):555-569, 1964.

[48] A. M. Cuitiño and M. Ortiz. Constitutive modeling of $\mathrm{L} 1_{2}$ intermetallic crystals. Materials Science and Engineering A, 170(1-2):111-123, 1993.

[49] Shahriyar Keshavarz and Somnath Ghosh. A crystal plasticity finite element model for flow stress anomalies in $\mathrm{Ni}_{3} \mathrm{Al}$ single crystals. Philosophical Magazine, 95(24):2639-2660, 2015.

[50] Christine Dianne Allan. Plasticity of nickel base single crystal superalloys. PhD thesis, Massachusetts Institute of Technology, 1995.

[51] George R. Weber and Somnath Ghosh. Thermo-mechanical deformation evolution in polycrystalline Ni-based superalloys by a hierarchical crystal plasticity model. Materials at High Temperatures, 33(4-5):401-411, 2016.

[52] Shahriyar Keshavarz, Somnath Ghosh, Andrew C E Reid, and Stephen A Langer. Acta Materialia A non-Schmid crystal plasticity finite element approach to multi-scale modeling of nickel-based superalloys. Acta Materialia, 114:106-115, 2016.

[53] K. Aoki and O. Izumi. Flow and fracture behaviour of $\mathrm{Ni}_{3}(\mathrm{Al}$.Ti) single crystals tested in tension. Journal of Materials Science, 14(8):1800-1806, 1979. 


\section{BIBLIOGRAPHY}

[54] Jianjun Wang, Wei Guo Guo, Yu Su, Ping Zhou, and Kangbo Yuan. Anomalous behaviors of a single-crystal Nickel-base superalloy over a wide range of temperatures and strain rates. Mechanics of Materials, 94:79-90, 2016.

[55] D. Cendon R. Sancho and F. Gálvez. Modelo del comportamiento mecánmico de materiales con cambios microestructurales. Anales de Mecánica de la Fractura, 33:423-428, 2016.

[56] Bertram Hopkinson. A method of measuring the pressure produced in the detonation of high, explosives or by the impact of bullets. Philosophical Transactions of the Royal Society of London. Series A, Containing Papers of a Mathematical or Physical Character, 213(497-508):437-456, 1914.

[57] Herbert Kolsky. An investigation of the mechanical properties of materials at very high rates of loading. Proceedings of the physical society. Section $B, 62(11): 676,1949$.

[58] J Harding, EO Wood, and JD Campbell. Tensile testing of materials at impact rates of strain. Journal of Mechanical Engineering Science, 2(2):88-96, 1960.

[59] RM Davies. A critical study of the hopkinson pressure bar. Philosophical Transactions of the Royal Society of London. Series A, Mathematical and Physical Sciences, 240(821):375-457, 1948.

[60] WW Chen and B Song. Split Hopkinson bar design, testing and applications. Mechanical engineering series, Springer, London, 2011.

[61] Rajeev Kapoor and Sia Nemat-Nasser. Determination of temperature rise during high strain rate deformation. Mechanics of materials, 27(1):1-12, 1998.

[62] L Pochhammer. On the propagation velocities of small oscillations in an unlimited isotropic circular cylinder. J. Reine Angewandte Math, 81:324, 1876 . 
[63] Ci Chree. The equations of an isotropic elastic solid in polar and cylindrical co-ordinates their solution and application. Transactions of the Cambridge Philosophical Society, 14:250, 1889.

[64] P.S. Follansbee; C. Frantz. Wave propagation in the Split Hopkinson Pressure Bar. Journal of Engineering Materials and Technology, 105:61$66,1983$.

[65] Zhouhua Li and John Lambros. Determination of the dynamic response of brittle composites by the use of the split hopkinson pressure bar. Composites Science and Technology, 59(7):1097-1107, 1999.

[66] Abaqus. Analysis User's Manual, version 6.14. Dassault Systemes, 2014.

[67] Pauli Virtanen, Ralf Gommers, Travis E. Oliphant, Matt Haberland, Tyler Reddy, David Cournapeau, Evgeni Burovski, Pearu Peterson, Warren Weckesser, Jonathan Bright, Stéfan J. van der Walt, Matthew Brett, Joshua Wilson, K. Jarrod Millman, Nikolay Mayorov, Andrew R. J. Nelson, Eric Jones, Robert Kern, Eric Larson, CJ Carey, İlhan Polat, Yu Feng, Eric W. Moore, Jake Vand erPlas, Denis Laxalde, Josef Perktold, Robert Cimrman, Ian Henriksen, E. A. Quintero, Charles R Harris, Anne M. Archibald, Antônio H. Ribeiro, Fabian Pedregosa, Paul van Mulbregt, and SciPy 1. 0 Contributors. SciPy 1.0: Fundamental Algorithms for Scientific Computing in Python. Nature Methods, 17:261-272, 2020.

[68] Kazuya Shinagawa, Toshihiro Omori, Katsunari Oikawa, Ryosuke Kainuma, and Kiyohito Ishida. Ductility enhancement by boron addition in co-al-w high-temperature alloys. Scripta Materialia, 61(6):612-615, 2009.

[69] S. Schänzer and E. Nembach. The critical resolved shear stress of $\gamma^{\prime}-$ strengthened nickel-based superalloys with $\gamma^{\prime}$-volume fractions between 0.07 and 0.47. Acta Metallurgica Et Materialia, 40(4):803-813, 1992.

[70] Shubhayu Sinharoy, Pauline Virro-Nic, and Walter W Milligan. Deformation and strength behavior of two nickel-base turbine disk alloys at $650^{\circ} \mathrm{C}$. Metallurgical and Materials Transactions A, 32(8):2021-2032, 2001. 


\section{BIBLIOGRAPHY}

[71] J Sato, T Omori, K Oikawa, I Ohnuma, R Kainuma, and K Ishida. Cobaltbase high-temperature alloys. Science, 312(5770):90-91, 2006.

[72] A Bauer, Steffen Neumeier, Florian Pyczak, and Mathias Göken. Microstructure and creep strength of different $\gamma / \gamma^{\prime}$-strengthened Co-base superalloy variants. Scripta Materialia, 63(12):1197-1200, 2010.

[73] TM Pollock, J Dibbern, M Tsunekane, J Zhu, and A Suzuki. New Cobased $\gamma-\gamma^{\prime}$ high-temperature alloys. JoM, 62(1):58-63, 2010.

[74] Michael S Titus, Akane Suzuki, and Tresa M Pollock. High temperature creep of new $\mathrm{L}_{2}$-containing cobalt-base superalloys. Superalloys, 12:823831, 2012.

[75] R Casas, F Gálvez, and M Campos. Microstructural development of powder metallurgy cobalt-based superalloys processed by field assisted sintering techniques (fast). Materials Science and Engineering: A, 724:461-468, 2018 .

[76] Rafael Casas Ferreras. Design of high temperature cobat-based alloys processed by powder metallurgy route. $\mathrm{PhD}$ thesis, $\mathrm{Ph}$. D. Tesis, Universidad Carlos III de Madrid, 2018.

[77] René41. https://www. neonickel.com/es/alloys/ aleaciones-de-niquel/rene-41/. Accessed: 2020-04-13.

[78] D. Cendón Franco F. Gálvez Díaz-Rubio and V. Sánchez Gálvez. Ensayos mecánicos en materiales a elevada velocidad de deformación y alta temperatura. Anales de Mecánica de la Fractura, 22:508-513, 2005.

[79] Stephen M Walley. The effect of temperature gradients on elastic wave propagation in split hopkinson pressure bars. Journal of Dynamic Behavior of Materials, pages 1-9, 2020.

[80] Akane Suzuki and Tresa M Pollock. High-temperature strength and deformation of $\gamma-\gamma^{\prime}$ two-phase co-al-w-base alloys. Acta Materialia, 56(6):1288-1297, 2008. 
[81] Yolita M Eggeler, Michael S Titus, Akane Suzuki, and Tresa M Pollock. Creep deformation-induced antiphase boundaries in $\mathrm{L}_{2}$-containing singlecrystal cobalt-base superalloys. Acta materialia, 77:352-359, 2014.

[82] Roger C Reed. The superalloys: fundamentals and applications. Cambridge university press, 2008.

[83] Yingbin Bao and Tomasz Wierzbicki. On fracture locus in the equivalent strain and stress triaxiality space. International Journal of Mechanical Sciences, 46(1):81-98, 2004.

[84] ASM International. ASM handbook. Volume 1, Properties and selection: irons, steels, and high-performance alloys. Materials Park, OH, ASM International, 1990.

[85] Vascomax c-250. http://www.dynamicmetals.net/VascoMaxCatalog. pdf. Accessed: 2020-07-13.

[86] Product data of $18-\mathrm{Ni}$ maraging steel, 250 grade. http://www. dynamicmetals.net/VascoMaxCatalog.pdf. Accessed: 2020-07-13.

[87] D Rittel, LH Zhang, and S Osovski. The dependence of the taylor-quinney coefficient on the dynamic loading mode. Journal of the Mechanics and Physics of Solids, 107:96-114, 2017.

[88] K Harris, GL Erickson, and RE Schwer. Mar m 247 derivations - cm 247 lc ds alloy, cmsx ${ }^{\circledR}$ single crystal alloys, properties and performance. In 5th Int. Symp, pages 221-230, 1984.

[89] Caroline A Schneider, Wayne S Rasband, and Kevin W Eliceiri. Nih image to imagej: 25 years of image analysis. Nature methods, 9(7):671, 2012.

[90] User manual-ecorr-digital image correlation tool. https://www.ntnu. edu/kt/ecorr. Accessed: 2019-07-01.

[91] Y Chen, AH Clausen, OS Hopperstad, and M Langseth. Application of a split-hopkinson tension bar in a mutual assessment of experimental tests 


\section{BIBLIOGRAPHY}

and numerical predictions. International Journal of Impact Engineering, 38(10):824-836, 2011.

[92] Murray Kaufman. Properties of cast Mar-M-247 for turbine blisk applications. In 5th International Symposium on Superalloys, pages 43-52, 1984.

[93] D Raabe. Modelling of active slip systems, Taylor factors and grain rotations during rolling and compresssion deformation of plycrystalline intermetallic $\mathrm{L}_{2}$ compounds. Acta Metallurgica et Materialia, 43(4):1531$1540,1995$.

[94] Michael E Kassner. Fundamentals of creep in metals and alloys. Butterworth-Heinemann, 2015.

[95] Daniel Peirce, Robert J Asaro, and A Needleman. Material rate dependence and localized deformation in crystalline solids. Acta metallurgica, 31(12):1951-1976, 1983.

[96] N. Bertin, L. Capolungo, and I. J. Beyerlein. Hybrid dislocation dynamics based strain hardening constitutive model. International Journal of Plasticity, 49:119-144, 2013.

[97] D. Peirce, R. J. Asaro, and A. Needleman. An analysis of nonuniform and localized deformation in ductile single crystals. Acta Metallurgica, 30(6):1087-1119, 1982.

[98] Michael A Groeber and Michael A Jackson. DREAM.3D: a digital representation environment for the analysis of microstructure in 3d. Integrating Materials and Manufacturing Innovation, 3(1):5, 2014.

[99] Michael Groeber, Somnath Ghosh, Michael D. Uchic, and Dennis M. Dimiduk. A framework for automated analysis and simulation of 3D polycrystalline microstructures. Part 2: Synthetic structure generation. Acta Materialia, 56(6):1274-1287, 2008.

[100] Guido Van Rossum and Fred L Drake Jr. Python reference manual. Centrum voor Wiskunde en Informatica Amsterdam, 1995. 
[101] Jacques Lacaze and Alain Hazotte. Directionally solidified materials: nickel-base superalloys for gas turbines. Textures and Microstructures, 13, 1990.

[102] Y. Z. Zhou and N. R. Green. Competitive grain growth in directional solidification of a nickel-base superalloy. Proceedings of the International Symposium on Superalloys, 56:317-324, 2008.

[103] JK Mackenzie and MJ Thomson. Some statistics associated with the random disorientation of cubes. Biometrika, 44(1-2):205-210, 1957.

[104] H. A Kuhn and H. G. Sockel. Comparison between experimental determination and calculation of elastic properties of nickel-base superalloys between 25 and 1200 C. Physica Status Solidi (a), 110(2):449-458, 1988.

[105] Sarra Haouala, Javier Segurado, and Javier LLorca. An analysis of the influence of grain size on the strength of FCC polycrystals by means of computational homogenization. Acta Materialia, 148:72-85, 2018.

[106] Jian Hong Liao, Hui Yun Bor, Chuen Guang Chao, and Tzeng Feng Liu. Effects of rhenium on microstructure and phase stability of MARM247 Ni-base fine-grain superalloy. Materials Transactions, 51(4):810$817,2010$.

[107] EO Hall. The deformation and ageing of mild steel: Iii discussion of results. Proceedings of the Physical Society. Section B, 64(9):747, 1951.

[108] NJ Petch. The cleavage strength of polycrystals. Journal of the Iron and Steel Institute, 174:25-28, 1953.

[109] Hsin Erh Huang and Chun Hao Koo. Characteristics and Mechanical Properties of Polycrystalline CM 247 LC Superalloy Casting. Materials Transactions, 45(2):562-568, 2004.

[110] F. Grytten, B. Holmedal, O. S. Hopperstad, and T. Børvik. Evaluation of identification methods for YLD2004-18p. International Journal of Plasticity, 24(12):2248-2277, 2008. 


\section{BIBLIOGRAPHY}

[111] H-J Bunge. Texture analysis in materials science: mathematical methods. Butterworth Co, 1982.

[112] Ted Belytschko. A Survey of Numerical Methods and Computer Programs for Dynamic Structural Analysis. Nuclear Engineering and Design, 13(2):258-283, 1976. 


\section{List of Tables}

3.1 Values of the parameters that define the yield strength evolution of the $\mathrm{L}_{2}$ intermetallic compounds. The first row is for $\mathrm{Ni}_{3} \mathrm{Al}$ and the second row for $\mathrm{Co}_{3}(\mathrm{Al}, \mathrm{W}) \ldots \ldots . \ldots 36$

3.2 Model parameters for the ternary alloy . . . . . . . . . . . . . 44

3.3 Model parameters for the quinary alloy . . . . . . . . . . . . . 44

3.4 Error (\%) between the experimental yield strengths and the model predictions for each temperature . . . . . . . . . . . . . . . 44

3.5 Chemical composition (\%wt.) of the alloy VascoMax C-250. Data obtained from the supplier. . . . . . . . . . . . 52

3.6 Model parameters for the steel VascoMax C-250 in the annealed condition. . . . . . . . . . . . . . . 56

3.7 Chemical composition of MAR-M247 (\%wt.). . . . . . . . . 59

$3.8 \quad 0.2 \%$ yield strength of MAR-M247 at testing strain rates and room temperature. . . . . . . . . . . . . 65

3.9 Material constants for MAR-M247. . . . . . . . . . . . . 69

4.1 Octahedral slip systems. . . . . . . . . . . . . . . . . . 75

4.2 Coefficients of the strength-interaction matrix $\mathrm{Q}^{\alpha \beta}$ for an FCC material. Each system ID is defined in Table 4.1 . . . . . . . 76

4.3 Parameters of the CP model for MAR-M247 . . . . . . . . . . 85

4.4 Variation on the yield strength as a function of grain size . . . . 97

4.5 Experimental $0.2 \%$-yield-strength values and numerical predictions for polycrystal and quasi-polycrystal MAR-M247 specimens. The predicted isotropic elastic constants are also compared with the values from literature $[104] . \ldots \ldots 7$ 


\section{List of Figures}

1.1 Illustration of the basic components of a jet engine. Adapted from $[4] \ldots \ldots \ldots \ldots \ldots \ldots$

1.2 Examples of yield-strength anomaly (YSA) in Ni- and Co-base (9Al-9W, 2Ta) superalloys. Obtained from [6]. . . . . . . . . 4

1.3 Evolution of the working temperature of nickel-base superalloys over the last decades [7]. . . . . . . . . . . . . . . . 4

2.1 Motion of an edge dislocation in a crystal. The edge dislocation can be imagined as an extra half-plane of atoms [26]. . . . . . . 10

2.2 Relation between the stress applied in an uniaxial tensile test and the resolved shear stress acting in the slip system. . . . . . 11

2.3 Representation of (a) the energy barrier that a dislocation, with length $l$, has to overcome to move from point $x_{1}$ to $x_{2}$ and (b) evolution of the macroscopically measured glide resistance as a function of temperature. The subscripts $a$ and $t$ stands for athermal and thermal components. . . . . . . . . . . .

2.4 Micromechanical basis of the multiplicative decomposition of the deformation gradient. Adapted from [28] . . . . . . . . . . 15

2.5 Different approaches for polycrystal homogenization. . . . . . . 17

2.6 (a) Typical $\gamma / \gamma^{\prime}$ microstructure of superalloys with rectangular $\gamma^{\prime}$ precipitates and (b) $\mathrm{L}_{2}$-type unit cell of $\mathrm{Ni}_{3} \mathrm{Al}$ intermetallic compound. . . . . . . . . . . . . . . .

2.7 Variation of the $\mathrm{Ni}_{3} \mathrm{Al}$ yield strength with temperature. Data

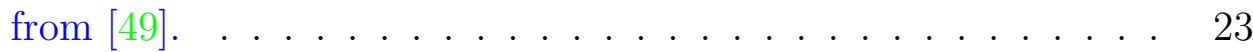


2.8 Kear-Wilsdorf locks. After cross-slipping, the APB and the dislocations are no longer coplanar, creating pinning points [48]. .

2.9 Schematic representation of the flow strength evolution with temperature in an alloy that exhibits YSA . . . . . . . . .

2.10 Scheme of the split-Hopkinson bar for the (a) compressive and (b) tensile configuration. . . . . . . . . . . . . . .

2.11 (a) Solution of the Pocchammer-Chree equation for a material with a Poisson's ratio $\nu=0.29$. The phase velocity is a function of the ratio between the bar radius $R$ and the wavelength $\Lambda$. (b) Dispersion of a trapezoidal pulse after travelling $1 \mathrm{~m}$ in a bar with $R=4.6 \mathrm{~mm}$ and $c_{0}=5178 \mathrm{~m} / \mathrm{s}$. More details in [64]. . . .

2.12 Comparison between the true stress-strain curves obtained from a SHPB experiment on aluminium with and without the use of a pulse shaper. . . . . . . . . . . . . . . .

3.1 Yield strength evolution of the (a) $\mathrm{Ni}_{3} \mathrm{Al}$ compound with temperature in [001] loading direction [49] and (b) $\mathrm{Co}_{3}(\mathrm{Al}, \mathrm{W})$ compound $[68] . \ldots \ldots \ldots \ldots \ldots \ldots$

3.2 Secondary-electron micrographs showing the $\gamma / \gamma^{\prime}$ microstructure of the (a) Co-12Al-10W alloy and of the (b) Co-12Al-10W-2Ti2Ta alloy (b). The cuboidal precipitates correspond to the $\gamma^{\prime}$ phase. Images by courtesy of Rafael Casas. . . . . . . . . . . .

3.3 Split-Hopkinson pressure bar device with the furnace for testing at high temperatures. . . . . . . . . . . . . . .

3.4 Adiabatic true stress-strain curves of (a) the ternary and (b) quinary alloys at $\dot{\mathrm{e}}=2500 \mathrm{~s}^{-1}$ for different temperatures. . . . .

3.5 Temperature dependence of the dimensionless yield strength of (a) the ternary and (b) quinary Co-base alloys at $\dot{\mathrm{e}}=2500 \mathrm{~s}^{-1}$. Dashed line fits the experimental data (black dots) with the proposed model. Dotted line only considers the thermal-softening part of the model (Johnson-Cook type behaviour). $\sigma_{\text {ref }}$ is the yield strength at room temperature, 2.02 GPa for the ternary alloy and $1.75 \mathrm{GPa}$ for the quinary alloy. . . . . . . . . . . 


\section{LIST OF FIGURES}

3.6 (a) Yield-strength comparison between the dynamic (present work) and quasi-static tests (other authors). (b) Estimation of the strain-rate sensitivity (slope of the straight line). . . . . . . .

3.7 Comparison between the force applied to the specimens during the experimental campaign and the one obtained from simulation. Data of the quinary alloy at $673 \mathrm{~K} \ldots$. . . . . . . .

3.8 Experimental (circle markers) and simulated (solid lines) true stress-strain curves, from $298 \mathrm{~K}$ to $873 \mathrm{~K}$, for the ternary (red) and quinary (black) Co-base superalloys under high-strain-rate loading $\left(2500 \mathrm{~s}^{-1}\right)$. Each sub-figure corresponds to a different temperature. . . . . . . . . . . . . . .

3.9 Experimental (circle markers) and simulated (solid lines) true stress-strain curves, from $973 \mathrm{~K}$ to $1123 \mathrm{~K}$, for the ternary (red) and quinary (black) Co-base superalloys under high-strain-rate loading $\left(2500 \mathrm{~s}^{-1}\right)$. Each sub-figure corresponds to a different temperature. . . . . . . . . . . . . . .

3.10 Frames of the virtual impact test: (a) before, (b) during impact and (c) after plate perforation . . . . . . . . . . .

3.11 Results of the virtual impact tests. Red dots represent the results without modelling the peak-flow stress while blue dots represent the results considering the peak-flow stress. . . . . . . . . . .

3.12 Metastable and equilibrium phases in the Fe-Ni system. Image obtained from [84]. . . . . . . . . . . . . . .

3.13 Adiabatic true stress-strain of the dynamic compression tests for the different temperatures: (a) $298 \mathrm{~K}$ and $473 \mathrm{~K}$, (b) $373 \mathrm{~K}, 573$ $\mathrm{K}, 635 \mathrm{~K}$ and $655 \mathrm{~K}$, (c) $683 \mathrm{~K}, 734 \mathrm{~K}$ and $895 \mathrm{~K}$ and (d) $800 \mathrm{~K}$ and 967 K. . . . . . . . . . . . . . .

3.14 Temperature dependence of the dimensionless yield strength of VascoMax C-250 in the annealed condition at $\dot{\mathrm{e}}=2500 \mathrm{~s}^{-1} \cdot \sigma_{\text {ref }}=1320 \mathrm{MPa}$ is the yield strength value at room temperature . . . . . . . 54

3.15 Hardness evolution of the maraging steel C-250 depending on the temperature and ageing time (precipitation heat treatment). Graph obtained from [84] . . . . . . . . . . . . . . . 54 
3.16 (a) Temperature dependence of the dimensionless yield strength of VascoMax C-250 in the annealed condition at $\dot{\mathrm{e}}=2500 \mathrm{~s}^{-1}$. $\sigma_{\text {ref }}=1320 \mathrm{MPa}$ is the yield strength value at room temperature. (b) Effect of the thermal softening due to adiabatic heating in the hardening law of the alloy at room temperature. . . . . . . .

3.17 Comparison between the adiabatic true stress-strain of the experimental and numerical dynamic compression tests for different temperatures: (a) $298 \mathrm{~K}$ and $473 \mathrm{~K}$, (b) $373 \mathrm{~K}, 573 \mathrm{~K}, 635 \mathrm{~K}$ and $655 \mathrm{~K}$, (c) $683 \mathrm{~K}, 734 \mathrm{~K}$ and $895 \mathrm{~K}$ and (d) $800 \mathrm{~K}$ and $967 \mathrm{~K} .58$

3.18 Microstructure of MAR-M247 DS alloy. The images (a) and (b) show the cross-section perpendicular to grain-growth direction and the image (c) in the parallel direction. . . . . . . . . . . . . 59

3.19 (a) Grain-size distribution of MAR-M247 DS alloy and (b) ellipse fitting of the grains to estimate the characteristic length $d_{g}$. . .

3.20 Geometry and dimensions of the smooth tensile samples employed in (a) quasi-static and $500 \mathrm{~s}^{-1}$ tests and (b) $150 \mathrm{~s}^{-1}$ tests.

3.21 Edge-tracing technique. (a) Initial frame and (b) frame just before failure. . . . . . . . . . . . . . . . . .

3.22 Quasi-static true stress-strain curves of MAR-M247 DS alloy along $0^{\circ}$ and $90^{\circ}$ direction. $\tau_{0}$ and $\Delta \varepsilon_{0}$ are confidential values suggested ITP Aero company. . . . . . . . . . . . . . .

3.23 Split-Hopkinson tension bar for tests at $150 \mathrm{~s}^{-1}$ (SIMLab). The strain gauges are in positions 1,2 and $3 . \ldots \ldots$. . . . .

3.24 Experimental set-up for tests at $150 \mathrm{~s}^{-1}$ strain rate with the highspeed camera. . . . . . . . . . . . . . . . .

3.25 Split-Hopkinson Tension Bar set-up for tests at $500 \mathrm{~s}^{-1}$ (UPM).

3.26 Dynamic true stress-strain curves of MAR-M247 DS alloy along $0^{\circ}$ and $90^{\circ}$ direction at an engineering strain rate of $150 \mathrm{~s}^{-1}$ and room temperature. $\tau_{0}$ and $\Delta \varepsilon_{\max }$ are confidential values suggested ITP Aero company. . . . . . . . . . . . . . . . 


\section{LIST OF FIGURES}

3.27 Experimental true stress-strain curves of the dynamic tests (500 $\mathrm{s}^{-1}$ ) at different temperatures for $0^{\circ}$ and $90^{\circ}$ orientations. $\tau_{0}$ and $\Delta \varepsilon_{\max }$ are confidential values suggested by ITP Aero company. $\Delta \varepsilon_{\max }=\Delta \varepsilon_{0} \ldots \ldots \ldots \ldots \ldots \ldots \ldots \ldots$

3.28 Temperature dependence of MAR-M247 yield strength along $0^{\circ}$ and $90^{\circ}$ direction. Markers depict the mean value with the error bar.

3.29 Finite element model used for numerical simulation of MARM247 under dynamic loading and different temperatures. . . . .

3.30 Numerical true stress-strain curves compared with the experimental data for dynamic tensile tests $\left(500 \mathrm{~s}^{-1}\right)$ at different temperatures. $\tau_{0}$ and $\Delta \varepsilon_{\max }$ are confidential values suggested by ITP Aero company. . . . . . . . . . . . . . . . . .

4.1 Illustration of the cylindrical FE models used to simulate the gauge length of the specimens. Each color represent a different grain and each sub-figure corresponds to a different sample orientation with respect to the grain-growth direction (a) $0^{\circ}$, (b) $30^{\circ}$, (c) $45^{\circ}$, (d) $60^{\circ}$, (e) $90^{\circ}$. Rotation angles with respect to the

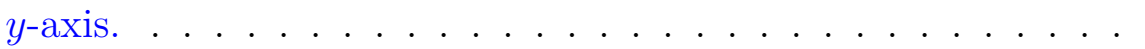

4.2 (a) Synthetic cubic volume and (b) comparison between the experimental and synthetic grain-size distribution. The dashed line represents the fitting of the experimental log-normal distribution (Section 3.4). . . . . . . . . . . . . . . .

4.3 Crystal orientation respect to the reference system . . . . . . . .

4.4 Stereographic projection of the crystallographic orientation for the (a) DS texture and (b) random orientation. 1000 grains were employed for the realizations. Plot (c) compares the PDF of the disorientation angle from the random realization to the theoretical value of perfect randomness [103] . . . . . . . . . . . . 
4.5 Some virtual $0^{\circ}$-oriented samples used in the simulations in the undeformed state $(\mathrm{a}, \mathrm{c})$ and after deformation $(\mathrm{b}, \mathrm{d})$. The contour plots show the stress concentration at grain boundaries. Images (a) and (b) are samples with $4 \mathrm{~mm}$ diameter while (c) and (d) are samples with $3 \mathrm{~mm}$ diameter. . . . . . . . . . . . .

4.6 Some virtual $90^{\circ}$-oriented samples used in the simulations in the undeformed state $(\mathrm{a}, \mathrm{c})$ and after deformation $(\mathrm{b}, \mathrm{d})$. The contour plots show the stress concentration at grain boundaries. Images (a) and (b) are samples with $4 \mathrm{~mm}$ diameter while (c) and (d) are samples with $3 \mathrm{~mm}$ diameter. . . . . . . . . . . .

4.7 Experimental (lines) and numerical (circles) true stress-strain curves for MAR-M247 DS alloy at room temperature under (a) quasi-static and (b, c) dynamic regimes and different loading directions. . . . . . . . . . . . . . .

4.8 Elastic part of the experimental (lines) and numerical (circles) true stress-strain curves for the quasi-static tests. . . . . . . . .

4.9 Amount of plastic slip $\Gamma$ accommodated by grains with different maximum Schmid factor for $90^{\circ}$ samples at the beginning of plastic deformation $\left(0.1 \Delta \varepsilon_{\max }\right)$, at the middle of the test $\left(0.45 \Delta \varepsilon_{\max }\right)$ and at the final stage $\left(0.9 \Delta \varepsilon_{\max }\right) \ldots \ldots . . . . . . .$.

4.10 Amount of elements with local stress (von-Mises stress) values $\bar{\sigma}_{\mu}$ larger than the global value supported by the sample $\bar{\sigma}$ for (a) $0^{\circ}$ - and (b) $90^{\circ}$-oriented samples. The data involve the last frame of all simulations. . . . . . . . . . . . . . . 91

4.11 Detailed view of the surface of a tested MAR-M247-DS sample. 92

4.12 Virtual specimen for simulating Kaufman tensile tests with 255 different equiaxed grains. . . . . . . . . . . . . . . . . . 94

4.13 Realistic RVE of MAR-M247. . . . . . . . . . . . . . 94

4.14 Experimental and numerical (prediction) engineering stress-strain curve for polycrystal MAR-M247. Experimental results from [106] were scaled down for $5.5 \mathrm{~mm}$ by using the Hall-Petch expression and data from [109]. . . . . . . . . . . . 96 


\section{LIST OF FIGURES}

4.15 Quasi-static true stress-strain curves for MAR-M247 DS alloy tested virtually at different angles with respect to grain-growth direction. The microstructure of the samples is shown in Figure

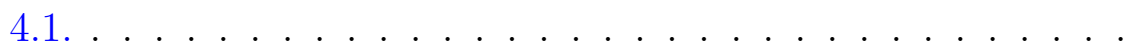

4.16 Prediction of the evolution of the normalised yield strength and Young's modulus with respect to loading direction. . . . . . . . 98

A.1 Reference and deformed configuration. Graphic representation of the polar decomposition of the deformation gradient. . . . . . 104

B.1 Axes rotations defined by Euler angles in Bunge convention. . . 106

C.1 $90^{\circ}$ samples with different element sizes. (a) $0.3 \times 0.3 \times 0.3 \mathrm{~mm}^{3}$, (b) $0.2 \times 0.2 \times 0.2 \mathrm{~mm}^{3}$ and (c) $0.15 \times 0.15 \times 0.15 \mathrm{~mm}^{3}$. . . . 108

C.2 True stress-strain curves for the same virtual specimen with different size of elements. $0.3 \times 0.3 \times 0.3 \mathrm{~mm}^{3}, 0.2 \times 0.2 \times 0.2 \mathrm{~mm}^{3}$ and $0.15 \times 0.15 \times 0.15 \mathrm{~mm}^{3} \ldots \ldots \ldots . \ldots 109$

C.3 Histogram representing the relative amount of elements with a certain level of local von Mises stress in the last frame of the simulation. (a) General plot and (b) same plot with a zoom-in for values larger than $1250 \mathrm{MPa}$ (dashed line in (a)). . . . . . . 109

C.4 Distribution of the von-Mises stress (MPa) on $90^{\circ}$ samples in the last frame of the simulation. (a) $0.3 \times 0.3 \times 0.3 \mathrm{~mm}^{3}$, (b) $0.2 \times$ $0.2 \times 0.2 \mathrm{~mm}^{3}$ and (c) $0.15 \times 0.15 \times 0.15 \mathrm{~mm}^{3}$. . . . . . 110

D.1 Sample. . . . . . . . . . . . . . . . . . 112

D.2 Reaction forces ( $y$ - component) versus time history on sample surfaces $y=0$ and $y=L$ using the explicit (black line) and dynamic implicit solver. . . . . . . . . . . . . . . . 113

D.3 (a) Stress evolution ( $y$ - component) along y-direction of the sample (loading direction) during the elastic loading for $0.0 \leq 0.002$ ms. Black lines correspond to the explicit solver while blue lines to the implicit one. (b) Difference (in \%) between the implicit and explicit solution. . . . . . . . . . . . . . . . 113 
D.4 a) Stress evolution ( $y$ - component) along y-direction of the sample (loading direction) during the elastic loading for $0.003 \leq$ $0.005 \mathrm{~ms}$. Black lines correspond to the explicit solver while blue lines to the implicit one. b) Difference (in \%) between the

D.5 a) Stress evolution ( $y$ - component) along loading direction for $0.0055 \leq 0.0075 \mathrm{~ms}$. Black lines correspond to the explicit solver while blue lines to the implicit one. b) Difference (in \%) between the implicit and explicit solution. . . . . . . . . . . . .

D.6 a) Stress evolution ( $y$ - component) along loading direction for $0.008 \leq 0.01 \mathrm{~ms}$. Black lines correspond to the explicit solver while blue lines to the implicit one. b) Difference (in \%) between the implicit and explicit solution.

D.7 a) Stress evolution ( $y$ - component) along loading direction for $0.0105 \leq 0.0125 \mathrm{~ms}$. Black lines correspond to the explicit solver while blue lines to the implicit one. b) Difference (in \%) between the implicit and explicit solution.

D.8 a) Stress evolution ( $y$ - component) along loading direction for $0.013 \leq 0.015 \mathrm{~ms}$. Black lines correspond to the explicit solver while blue lines to the implicit one. b) Difference (in \%) between the implicit and explicit solution. . . . . . . . . . . . . . . 116 